\title{
Development of a Planar Shipboard Skid-equipped Rotary-wing Aircraft Manoeuvring and Securing Simulation
}

\author{
by \\ Alexander R. Schock \\ A thesis submitted to \\ the Faculty of Graduate and Postdoctoral Affairs \\ in partial fulfilment of \\ the requirements for the degree of \\ Master of Applied Science \\ in \\ Mechanical Engineering \\ Ottawa-Carleton Institute for Mechanical and Aerospace Engineering \\ Department of Mechanical and Aerospace Engineering \\ Carleton University \\ Ottawa, Ontario, Canada \\ September 2020 \\ Copyright (C) \\ 2020 - Alexander R. Schock
}




\section{Abstract}

The capacity of unmanned aircraft systems (UAS) to operate in environments that are hazardous to humans, their preclusion of human error during operations, and progressively-declining operating costs motivate their increasing use in maritime shipboard operations in severe weather. Their high thrust-to-weight ratio and typical stiff 'skid-type' landing gear constrain mechanical securing and traversing options, and present complex ship-helicopter interface behaviour which must be characterized.

A securing and traversing device concept has been developed in parallel with the development of the planar case of SRAMSS (Skid-equipped Rotary-wing Aircraft Manoeuvring and Securing Simulation). The planar case aims to verify the system formulation for the dynamic interface analysis of skid-equipped rotary-wing aircraft during shipboard operations in elevated sea and weather conditions.

SRAMSS integrates four major techniques to achieve completeness of the simulation. First, SRAMSS models the aircraft as a fully-planar mass-coupled rigid airframe and dynamic flexible finite element landing gear. Second, direct method dynamic finite element modelling is used to characterize infinitely-variable skid-type landing gear, which is adapted for use in Kane's method, and demonstrates a powerful technique for merging rigid-body and flexible-body dynamics. Third, complex ship-aircraft contact is modelled by a Separation Axis Theorem algorithm with dynamic response capabilities, including a LuGre frictional slider model. Aerodynamic wind-induced drag, and blade element rotor disc models complete the modelling of the embarked aircraft.

Thorough verification of the planar version of SRAMSS confirms the proper implementation, and simultaneous functioning of the included models. Preliminary validation of the rotor blade element model against computational and experimental data indicates the need for refinement. Nevertheless, the planar case of SRAMSS verifies a suitable system formulation for a state-of-the-art fully-spatial dynamic interface analysis simulation for skid-equipped rotary-wing aircraft. 
Dedicated to the reader, who may find valuable insight, or simply the enjoyment of the content herein. 


\section{Acknowledgements}

I would like to sincerely thank all of the people who have unconditionally supported me through to the end of this research.

First and foremost, I would like to thank my supervisor Dr. Robert G. Langlois for his invaluable guidance and the wealth of knowledge imparted to me. Rob has afforded me the freedom to progress my research in the directions I saw fit, and has equitably received and debated the ideas I have brought forward.

I would also like to thank my family, friends, and loved ones for their relentless encouragement and support, even in the most demanding of times during my research.

Further, I would like to thank my fellow researchers for their exchange of knowledge and ideas, lending their time, and sharing in the struggles of the typical graduate student.

Finally I would like to thank Carleton University's Department of Mechanical and Aerospace Engineering and the Faculty of Graduate and Postdoctoral Affairs for the opportunity to pursue graduate studies and complete this research.

Thank you. 


\section{Contents}

Abstract $\quad$ i

Acknowledgements $\quad$ iii

Contents $\quad$ iv

List of Figures vii

List of Tables $\quad$ xi

List of Symbols $\quad$ xii

Acronyms $\quad$ xix

1 Introduction $\quad 1$

1.1 Shipboard Securing and Manoeuvring Systems . . . . . . . . . . . 2

1.2 Shipboard Helicopter Modelling . . . . . . . . . . . . . . . 4

1.2.1 Ship Deck Motion . . . . . . . . . . . . . . 4

1.2.2 Securing and Manoeuvring Models . . . . . . . . . . . 5

1.2.3 Skid-type Landing Gear Models . . . . . . . . . . . . . . . . . 7

1.2.4 Aerodynamic Ship-helicopter Interactions . . . . . . . . . . 8

1.2.5 Rotary-wing Aerodynamics Modelling . . . . . . . . . . 8

1.3 Contact Modelling . . . . . . . . . . . . . . . . . . 9

1.4 Friction Modelling . . . . . . . . . . . . . . . . . . . 10

1.5 Thesis Objectives . . . . . . . . . . . . . . . . . 11

1.6 Thesis Overview . . . . . . . . . . . . . . . . . 13

2 System Description 15

2.1 Securing and Manoeuvring System Conceptual Design . . . . . . . . . 15 
2.1.1 Securing and Manoeuvring Mechanism Requirements . . . . . 16

2.1.2 Skid-type Securing and Manoeuvring Concept . . . . . . . . . 16

2.2 Mathematical Conventions and Methods . . . . . . . . . . . . . . 17

2.3 Dynamic System Definition . . . . . . . . . . . . . . . . 24

3 System Kinematics $\quad 32$

3.1 Body Kinematics . . . . . . . . . . . . . . . . . . . . . . 33

3.2 Polygon Kinematics . . . . . . . . . . . . . . . . . . . . . . . . 42

4 System Dynamics $\quad 47$

4.1 Dynamic Finite Element Model . . . . . . . . . . . . . . . . . . 48

4.2 Contact Dynamics Model . . . . . . . . . . . . . . . . 57

4.3 Aerodynamic Models . . . . . . . . . . . . . . . . . . 66

4.3.1 Aerodynamic Body Drag Forces . . . . . . . . . . . . . . 67

4.3.2 Aerodynamic Rotor Disc Forces . . . . . . . . . . . . . . . 68

4.4 Other Force-generating Elements . . . . . . . . . . . . . . 74

4.4.1 Flexible-Rigid Body Interface Model . . . . . . . . . . . . . . 75

4.4.2 External Damper Model . . . . . . . . . . . . . . 76

4.4.3 Gravitational Model ... . . . . . . . . . . . . 79

4.5 Inertial Forces . . . . . . . . . . . . . . . . . . . . . . 79

4.6 Summary of Kinetic Quantities . . . . . . . . . . . . . . . 80

5 System Assembly and Simulation Architecture 82

5.1 System Assembly . . . . . . . . . . . . . . . . . . . 82

5.2 Simulation Architecture . . . . . . . . . . . . . . . 88

5.2.1 State Propagation . . . . . . . . . . . . . . . 89

5.2 .2 Contact Event Handling . . . . . . . . . . . . . . . . 90

5.2.3 Subroutine Architecture . . . . . . . . . . . . . . 94

6 SRAMSS Verification and Validation $\quad 97$

6.1 Generic Aircraft Description . . . . . . . . . . . . . . . 97

6.2 Test Cases Description . . . . . . . . . . . . . . . . . . 102

6.3 Test Cases Verification and Validation . . . . . . . . . . . . . . 107

6.3.1 Case 1: Gravitational Model . . . . . . . . . . . . . . . 107

6.3.2 Case 2: External Damper Model . . . . . . . . . . . . . . . . 108

6.3.3 Case 3: Aircraft Centre of Gravity . . . . . . . . . . . . . . . 111 
6.3.4 Case 4: Landing Gear DFE Model . . . . . . . . . . . . . 113

6.3.5 Case 5: Contact Dynamics Model . . . . . . . . . . . . . . . . 119

6.3.6 Case 6: Aerodynamics Models . . . . . . . . . . . . . 126

6.3.7 Case 7: Securing Concept Verification . . . . . . . . . . . . 136

6.3.8 Case 8: Comprehensive Phenomena Verification . . . . . . . . 141

6.4 General Remarks . . . . . . . . . . . . . . . . . . . . . . 150

7 Conclusion $\quad 153$

7.1 Completed Work . . . . . . . . . . . . . . . . . . 153

7.2 Recommendations . . . . . . . . . . . . . . . . . . . 155

7.3 Future Work . . . . . . . . . . . . . . . . . . . 157

$\begin{array}{ll}\text { References } & 158\end{array}$

Appendix A SRAMSS I/O File Structure 165

A.1 Input Files . . . . . . . . . . . . . . . . . 165

A.2 Output Files . . . . . . . . . . . . . . . . . . . 171

Appendix B Case 6 Prescribed Aerodynamics 172

B.1 Case 6.2: Rotor Thrust without Wind . . . . . . . . . . . . 172

B.2 Case 6.3: Rotor Thrust with Head-wind . . . . . . . . . . . 173

B.3 Case 6.4:Rotor Thrust with Head-wind and Cyclic Compensation . . 174

Appendix C Case 6 Experimental Blade Profiles $\quad 175$

C.1 Case 6.5 S-76 Experimental Blade Profile . . . . . . . . . . . . 175

C.2 Case 6.5 S-76 SRAMSS Blade Profile . . . . . . . . . . . 178

Appendix D Case 8 Prescribed Parameters 180

D.1 Case 8: Prescribed Aerodynamics . . . . . . . . . . . . . 180

D.2 Case 8: Prescribed Motions . . . . . . . . . . . . . . . 181

Appendix E Simulation Statistics 183

E.1 Case $8: 18 / 20 / 160 \ldots \ldots \ldots \ldots \ldots \ldots$ 


\section{List of Figures}

1.1 SH-60 Seahawk hovering over ship deck landing zone equipped with LAMPS III RAST system [1] . . . . . . . . . . . . . . . . . 2

1.2 Recovery Assist, Secure and Traverse (RAST) system [1] . . . . . . . 3

1.3 Aircraft Ship Integrated Secure and Traverse (ASIST) system [2]. . . 3

1.4 System description of Blackwell and Feik [3]. . . . . . . . . . 5

1.5 Force-generating elements included in Dynaface ${ }^{\circledR}[4] \ldots \ldots$. . . . . . 6

1.6 System description of Heliman [5] . . . . . . . . . . . . . . 6

1.7 Skid-type landing gear description $[6] \ldots \ldots \ldots \ldots \ldots$

2.1 Planar representation of the skid-equipped securing and manoeuvring system. . . . . . . . . . . . . . . . . . . 17

2.2 Planar description of SRAMSS simulation environment. . . . . . . . 25

2.3 System frames of reference. . . . . . . . . . . . . . . . . 26

2.4 Aircraft frames of reference. . . . . . . . . . . . . . . . 27

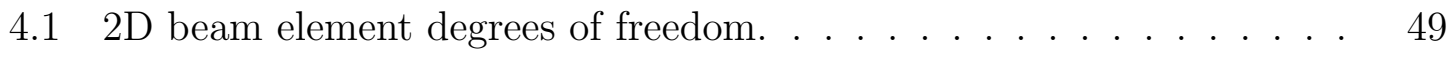

4.2 Arbitrarily-oriented 2D beam element frames of reference. . . . . . 51

4.3 Arbitrary axis separating two polygons. . . . . . . . . . . 58

4.4 Separation of polygons $P$ and $Q$ along surface normal axis ${ }_{N}^{P} \hat{\boldsymbol{s}}_{1} \ldots \quad \ldots 9$

4.5 Separation between DFE node $n_{j_{c}}$ and defined polygon $P$. . . . 59

4.6 Surface normal axes ${ }_{N}^{S} \hat{s}_{m}$ for the ship polygon $S \ldots \ldots \ldots \ldots$

4.7 Surface penetration penalty function diagram. . . . . . . . . . 62

4.8 LuGre friction bristle model. . . . . . . . . . . . . . . . . . 64

4.9 Rotor disc blade element model for generic aircraft. . . . . . . . . . . 69

4.10 Rotor disc BEM mesh. . . . . . . . . . . . . . . . . . 70

4.11 Rotor blade element airflow and forces diagram. . . . . . . . 71

4.12 Interface of DFE nodes to the airframe. . . . . . . . . 75 
4.13 Discrete external damper mounting locations. . . . . . . . . . . . . 77

5.1 State propagation with root-finding step termination. . . . . . . . . 90

5.2 SRAMSS program architecture. . . . . . . . . . . 95

6.1 Generic aircraft used in verification an validation test cases. . . . . . 98

6.2 Generic aircraft blade profile. . . . . . . . . . . . . . . . 101

6.3 Case 1 Aircraft CoG y-position and y-acceleration with respect to the inertial frame. . . . . . . . . . . . . . . . . . . . . . 107

6.4 Case 2 Airframe vertical response due to discrete external linear damper property variation. . . . . . . . . . . . . . . . . . . 109

6.5 Case 2 Landing gear vertical response due to discrete external linear damper property variation. . . . . . . . . . . . . . . . . . . 110

6.6 Case 2 Landing gear horizontal response due to discrete external linear damper property variation. . . . . . . . . . . . . . . . . 110

6.7 Case 3 Airframe roll-angle response to shifted CoGs. . . . . . . . . . 111

6.8 Case 3 Steady-state aircraft roll versus airframe CoG x-offset. . . . . 112

6.9 Case 3 Contact node normal forces versus airframe CoG offset. . . . . 113

6.10 Case 4 DFE model mesh configurations. . . . . . . . . . . . . . 115

6.11 Case 4.1 Aircraft response for fixed landing gear with DFE model mesh refinements. . . . . . . . . . . . . . . . . 116

6.12 Case 4.1 DFE model node construction. . . . . . . . . . . . . . 116

6.13 Case 4.2 Aircraft response for hinged landing gear with DFE model mesh refinements. . . . . . . . . . . . . . . . . . . . . 118

6.14 Case 5.1 Steady-state simulation configurations for normal force evaluation. . . . . . . . . . . . . . . . . . . . . . . 119

6.15 Case 5.2 Sliding velocity of leading (right) landing gear foot . . . . 121

6.16 Case 5.2 Trailing $\left(n_{1}\right)$ and leading $\left(n_{6}\right)$ landing gear feet frictional forces 122

6.17 Case 5.3 Oriented dynamic response simulation configurations. . . . . 123

6.18 Case 5.3 Position of airframe and contact nodes in the 'RSD push' scenario. . . . . . . . . . . . . . . . . . . . . 124

6.19 Case 5.3 Position of airframe and contact nodes in the 'RSD drag' scenario. . . . . . . . . . . . . . . . . . 125

6.20 Case 5.3 Position of airframe and contact nodes in the 'RSD hook' scenario. . . . . . . . . . . . . . . . . 126 
6.21 Case 6.1 Aircraft horizontal sliding velocity due to induced drag. . . . 127

6.22 Case 6.1 Aircraft vertical acceleration due to induced drag. . . . . . . 128

6.23 Case 6.2 Rotor disc thrust and airframe CoG height above the RSD deck. . . . . . . . . . . . . . . . . . . . . . 129

6.24 Case 6.3 Rotor disc thrust and rolling moment, and airframe CoG position relative to the RSD deck. . . . . . . . . . . . . . . . 130

6.25 Case 6.4 Rotor disc thrust and rolling moment, and airframe CoG position relative to the RSD deck. . . . . . . . . . . . . . . . . 132

6.26 Case 6.5 Comparison of blade section thrust coefficient between SRAMSS BEM model and CFD analysis from Garcia and Barakos. . . . . . . . 133

6.27 Case 6.5 Comparison of FoM for SRAMSS BEM and CFD model. $M_{t i p}=0.65, \theta_{t}=\left\{4^{\circ}, 5^{\circ}, 6^{\circ}, 7^{\circ}, 8^{\circ}, 9^{\circ}\right\} \ldots \ldots \ldots \ldots$

6.28 Case 6.5 Comparison of FoM for SRAMSS BEM and CFD model. $M_{\text {tip }}=0.60, \theta_{t}=\left\{6.5^{\circ}, 7.5^{\circ}, 8.5^{\circ}, 9.5^{\circ}\right\} \ldots \ldots \ldots$

6.29 Case 7.1 Static aircraft securing simulation configurations. . . . . . . 137

6.30 Case 7.1 Static aircraft securing in the 'On-centre' scenario. . . . . . 138

6.31 Case 7.1 Static aircraft securing in the 'Off-centre' scenario. . . . . . 138

6.32 Case 7.1 Static aircraft securing in the 'Rolled Ship' scenario. . . . . . 139

6.33 Case 7.2 Dynamic aircraft securing simulation configurations. . . . . . 139

6.34 Case 7.2 Dynamic aircraft securing in the 'Rolling Ship' scenario. . . 140

6.35 Case 7.2 Dynamic aircraft securing in the 'RSD Manoeuvre' scenario. 140

6.36 Case 7.2 Contact force magnitudes in the 'Rolling Ship' scenario. . . 141

6.37 Case 7.2 Contact force magnitudes in the 'RSD Manoeuvre' scenario. 141

6.38 Case 8 Comprehensive simulation time-stamped event animations. . . 145

6.39 Case 8 Airframe $\mathrm{CoG}$ position versus time for all combinations of $n_{n} / N_{\psi} / N_{e} \ldots \ldots \ldots \ldots \ldots$. . . . . . . . . . . . 146

6.40 Case 8 Aircraft distances relative to RSD points. . . . . . . . . . . . . 147

6.41 Case 8 Contact force magnitudes at each contact node. . . . . . . . . 148

6.42 Case 8 Rotor disc force magnitudes. . . . . . . . . . . . . . . . . . . . 149

B.1 Case 6.2 Prescribed aerodynamic values. . . . . . . . . . . . . . . . . 172

B.2 Case 6.3 Prescribed aerodynamic values. . . . . . . . . . . . . . 173

B.3 Case 6.4 Prescribed aerodynamic values. . . . . . . . . . . . . . . . 174

C.1 S-76 model rotor blade profile [7]. . . . . . . . . . . . . . . . . 176 
C.2 S-76 model rotor blade profile $[8] . \ldots \ldots \ldots \ldots \ldots \ldots$

C.3 Case 6.5 SRAMSS S-76 model rotor blade profile. . . . . . . . . . 178

D.1 Case 8 Prescribed aerodynamic values. . . . . . . . . . . . . 181

D.2 Case 8 Prescribed polygon motions. . . . . . . . . . . . . . . 182 


\section{List of Tables}

1.1 Shipboard securing and manoeuvring modelling summary . . . . . . . 12

4.1 Contact forces summary . . . . . . . . . . . . . . . 65

4.2 Complete system forces summary . . . . . . . . . . . . . . . . 81

6.1 Generic aircraft parameters . . . . . . . . . . . . . . . 99

6.2 DFE node functionality for generic aircraft . . . . . . . . . . . . . . . 99

6.3 Rotor disc blade element model parameters . . . . . . . . . . . . . . . 100

6.4 Contact dynamics parameters . . . . . . . . . . . . . 100

6.5 SRAMSS verification and validation test cases . . . . . . . . . 103

6.6 Case 1 Undeformed versus steady-state helicopter posture . . . . . . . 108

6.7 Case 2 Discrete damper parameter variations . . . . . . . . . . . . 109

6.8 Modified DFE model parameters for use in Case 4 . . . . . . . . . . . 114

6.9 Case 5.1 Contact normal forces at static equilibrium . . . . . . . . . . 120

6.10 Modified aircraft simulation parameters for use in Sub-case 5.2 . . . . 121

C.1 SRAMS S-76 model BEM parameters . . . . . . . . . . . . . 179 


\section{List of Symbols}

Mathematical Notations and Operators:

\begin{tabular}{|c|c|}
\hline $\mathbb{R}$ & Real number \\
\hline $\mathbb{Z}$ & Integer \\
\hline$\rightarrow$ & Physical vector quantity \\
\hline r & Unit vector quantity \\
\hline$\frac{\mathrm{d}}{\mathrm{d} t}(\quad)$ & Time derivative of expression in parenthesis \\
\hline & First time derivative \\
\hline & Second time derivative \\
\hline | | & Absolute value \\
\hline$\|\quad\|$ & Magnitude (Euclidean norm) \\
\hline & Skew-symmetric matrix representation \\
\hline$\circ$ & Hadamard product (element-wise multiplication) \\
\hline 1 & Adjusted value \\
\hline & Vector component along $x$ axis of $2 \mathrm{D}$ frame \\
\hline & Vector component along $y$ axis of 2D frame \\
\hline a & Vector component about $z$ axis of $2 \mathrm{D}$ frame \\
\hline$\left.\right|_{x, 3 D}$ & Vector component along $x$ axis of 3D frame \\
\hline & Vector component along $y$ axis of 3D frame \\
\hline$\left.\right|_{z, 3 D}$ & Vector component along $z$ axis of 3D frame \\
\hline
\end{tabular}

Frames of Reference:

$\mathrm{N} \quad$ Inertial frame of reference (origin at mean ship speed and heading)

$\mathrm{H} \quad$ Helicopter frame of reference (origin at airframe CoG) 
G Landing gear finite element model global frame

$\mathrm{n}_{\mathrm{j}} \quad$ Landing gear $j$ th finite element node frame of reference (aligned to G-frame)

$\mathrm{R} \quad$ Rotor disc frame of reference

S Ship deck polygon frame of reference

D RSD deck polygon frame of reference

CL Left wedge clamp polygon frame of reference

CR Right wedge clamp polygon frame of reference

\section{Indices:}

$d \quad d$ th external external damper

$h \quad h$ th rotor blade element

$i \quad i$ th generalized coordinate and generalized speed

$j \quad j$ th dynamic finite element node, $\forall j \in\left\{1, \ldots, N_{n}\right\}$

$j_{c} \quad j_{c}$ th dynamic finite element node considered for contact, $j_{c} \subseteq j$

$j_{d} \quad j_{d}$ th dynamic finite element node with attached external damper, $j_{d} \subseteq j$

$j_{u} \quad j_{u}$ th dynamic finite element node interfacing with the airframe, $j_{u} \subseteq j$

$k \quad k$ th body in the complete system

$l \quad l$ th rotor disc sector

$m \quad m$ th vertex of a polygon

$r \quad r$ th generic summation element index

Variables:

A Finite element cross-sectional area

$\boldsymbol{A}_{e q} \quad$ Equivalent flat-plate area

$[\boldsymbol{A}] \quad$ System assembly matrix

$[\boldsymbol{A}(t)] \quad$ Penetration state matrix

$\boldsymbol{B} \quad$ System assembly vector

$[\boldsymbol{B}(t)] \quad$ Penetration state change matrix

$C L_{m} \quad m$ th left wedge clamp polygon vertex

$C R_{m} \quad m$ th right wedge clamp polygon vertex 
$c_{h} \quad$ Rotor blade element chord length

$C_{d} \quad$ External damper linear damping coefficient

$C_{f} \quad$ Friction micro-displacement linear damping coefficient

$C_{p} \quad$ Penetration linear damping coefficient

$C_{L_{h l}} \quad$ Rotor blade element lift coefficient

$C_{L_{h, \alpha=0}}$ Rotor blade element zero angle of attack lift coefficient

$\frac{\mathrm{d} C_{L}}{\mathrm{~d} \alpha_{h l}} \quad$ Lift coefficient gradient with respect to angle of attack

$C_{D_{h l}} \quad$ Rotor blade element drag coefficient

$C_{D_{h, \alpha=0}}$ Rotor blade element zero angle of attack drag coefficient

$\frac{\mathrm{d} C_{D}}{\mathrm{~d} \alpha_{h l}} \quad$ Drag coefficient gradient with respect to angle of attack

C System assembly vector

$[\boldsymbol{C}] \quad$ Dynamic finite element total structure proportional damping matrix

$D_{m} \quad m$ th RSD deck polygon vertex

$[\boldsymbol{D}] \quad$ System assembly matrix

$[\boldsymbol{D}(t)] \quad$ Contact surface matrix

E Finite element Young's modulus

$F_{d} \quad$ Dynamic friction force

$F_{d_{d}} \quad d$ th external damper force

$F_{\text {LuGre }} \quad$ LuGre model friction force

$F_{i} \quad i$ th generalized active force

$F_{i}^{*} \quad i$ th generalized inertial force

$F_{s} \quad$ Static friction force

$\boldsymbol{F} \quad$ System generalized active forces

$\boldsymbol{F}^{*} \quad$ System generalized inertia forces

$\boldsymbol{F}_{G} \quad$ Finite element external nodal forces expressed in the global element frames

$\boldsymbol{F}_{l} \quad$ Finite element external nodal forces expressed in the local element frames

$\boldsymbol{F}_{\text {sys }} \quad$ Complete system forcing vector

$g(t) \quad$ Event root function

$I_{H} \quad$ Airframe inertia

I Finite element second moment of area

$K_{d} \quad$ External damper linear stiffness coefficient

$K_{f} \quad$ Friction micro-displacement linear stiffness coefficient

$K_{p} \quad$ Penetration linear stiffness coefficient

$[\boldsymbol{K}] \quad$ Dynamic finite element total structure stiffness matrix 
$\left[\boldsymbol{K}_{l}\right] \quad$ Element stiffness matrix expressed in local element frames

$\left[\boldsymbol{K}_{G}\right] \quad$ Element stiffness matrix expressed in globally-aligned element frames

$L \quad$ Finite element length

$m_{H} \quad$ Airframe mass

$M_{\text {tip }} \quad$ Rotor blade tip Mach number

$M_{h l} \quad$ Rotor blade element Mach number

$M_{d} \quad d$ th external damper airframe mount

$[\boldsymbol{M}] \quad$ Dynamic finite element total structure consistent mass matrix

$\left[\boldsymbol{M}_{l}\right] \quad$ Element consistent mass matrix expressed in local element frames

$\left[\boldsymbol{M}_{G}\right] \quad$ Element consistent mass matrix expressed in globally-aligned element frames

$\left[\boldsymbol{M}_{\text {sys }}\right] \quad$ Complete system mass matrix

$N_{b} \quad$ Number of rotor blades

$N_{B} \quad$ Number of bodies in the system

$N_{d} \quad$ Number of external dampers

$N_{e} \quad$ Number of rotor blade elements

$N_{g c} \quad$ Number of generalized coordinates in the system

$N_{n} \quad$ Number of dynamic finite element nodes

$N_{P} \quad$ Number of polygons

$N_{u} \quad$ Number of dynamic finite element interface nodes

$N_{s, P} \quad$ Number of surfaces in polygon $P$

$N_{v, P} \quad$ Number of vertices in polygon $P$

$N_{\psi} \quad$ Number of rotor disc azimuth selectors

$[\boldsymbol{N}] \quad$ Polynomial interpolation shape functions matrix

$\overrightarrow{\boldsymbol{o}} \quad$ Object contact point

$P \quad$ Generic polygon $P$

$P_{m} \quad m$ th generic polygon $P$ vertex

$q_{i} \quad i$ th generalized coordinate

$\dot{q}_{i} \quad i$ th generalized coordinate time-derivatives

$\boldsymbol{q} \quad$ System generalized coordinates

$\dot{\boldsymbol{q}} \quad$ System generalized coordinate time-derivatives

$\dot{\boldsymbol{q}}_{H} \quad$ Airframe generalized coordinate time-derivatives

$\dot{\boldsymbol{q}}_{n} \quad$ Dynamic finite element node generalized coordinate time-derivatives

$r \quad$ Radial position on rotor blade 
$\mathrm{d} r \quad$ Rotor blade element width

$R_{b} \quad$ Rotor blade radius

$R_{i} \quad$ Inner effective rotor blade radius as a percentage of rotor blade radius

$R_{o} \quad$ Outer effective rotor blade radius as a percentage of rotor blade radius

$\overrightarrow{\boldsymbol{R}}^{k} \quad k$ th body active force

$\overrightarrow{\boldsymbol{R}}^{* k} \quad k$ th body inertial force

$S_{m} \quad m$ th ship deck polygon vertex

$t_{\text {curr }} \quad$ Time of current state propagation

$t_{e} \quad$ Time of detected simulation event

$t_{\text {ref }} \quad$ Simulation time reference

$t_{\text {sol }} \quad$ End time of last successfully propagated solution

$\overrightarrow{\boldsymbol{T}}^{k} \quad k$ th body active torque

$\overrightarrow{\boldsymbol{T}}^{* k} \quad k$ th body inertial torque

$t / c_{h} \quad$ Rotor blade element thickness-to-chord ratio

$u_{a} \quad$ Axial displacement of node $a$ for finite element spanning from node $a$ to node $b$

$u_{b} \quad$ Axial displacement of node $b$ for finite element spanning from node $a$ to node $b$

$u_{i} \quad i$ th generalized speed

$U \quad$ Total volume strain energy

$U_{0} \quad$ Strain energy per unit volume

$\boldsymbol{u} \quad$ System generalized speeds

$\dot{u}_{i} \quad i$ th generalized speed time-derivative

$\boldsymbol{u}_{n} \quad$ Dynamic finite element node generalized speeds

$\dot{u} \quad$ Generalized speed time-derivatives

$\dot{\boldsymbol{u}}_{P} \quad$ Polygon generalized speed time-derivatives

$\dot{\boldsymbol{u}}_{H} \quad$ Airframe generalized speed time-derivatives

$\dot{\boldsymbol{u}}_{G} \quad$ Global finite element origin generalized speed time-derivatives

$\dot{\boldsymbol{u}}_{n} \quad$ Dynamic finite element node generalized speed time-derivatives

$v_{0_{h l}} \quad$ Rotor blade element axial flow velocity

$v_{1_{h l}} \quad$ Rotor blade element flow velocity

$v_{2_{h l}} \quad$ Rotor blade element tangential flow velocity

$v_{a} \quad$ Shear displacement of node $a$ for finite element spanning from node $a$ to node $b$ 
$v_{b} \quad$ Shear displacement of node $b$ for finite element spanning from node $a$ to node $b$

$v_{c_{h l}} \quad$ Rotor blade element climb velocity

$v_{i_{h l}} \quad$ Rotor blade element induced flow velocity

$v_{\text {rel }} \quad$ Relative sliding velocity of contact node along contact surface

$v_{s} \quad$ Stribeck velocity threshold

$v_{\text {wind,x }} \quad$ Longitudinal wind velocity component

$v_{w i n d, y} \quad$ Lateral wind velocity component

$v_{w i n d, z} \quad$ Vertical wind velocity component

${ }_{k} \boldsymbol{V}^{u_{i}} \quad$ Linear partial contribution along $u_{i}$ to kinematic quantity of body $k$, expressed in the $k$ th frame

$[\boldsymbol{V}] \quad$ Linear partial velocity matrix

$\left[\boldsymbol{V}_{n}\right] \quad$ Array of dynamic finite element node linear and angular partial velocity matrices

$\left[\boldsymbol{V}_{u}\right] \quad$ Array of dynamic finite element node linear and angular partial velocity matrices for interface nodes

$[\boldsymbol{W}] \quad$ Angular partial velocity matrix

$\boldsymbol{x}_{\text {sys }} \quad$ System solution

$\dot{\boldsymbol{x}} \quad$ State derivative vector

$\overrightarrow{\boldsymbol{Y}}_{k} \quad k$ th body remainder angular acceleration terms

$\boldsymbol{Y}_{n} \quad$ Array of dynamic finite element node remainder angular acceleration terms

$\vec{Z}_{k} \quad k$ th body remainder linear acceleration terms

$\boldsymbol{Z}_{n} \quad$ Array of dynamic finite element node remainder linear acceleration terms

$\alpha_{h l} \quad$ Rotor blade element angle-of-attack

$\alpha_{0} \quad$ Proportional damping mass constant

$\alpha_{1} \quad$ Proportional damping stiffness constant

$\boldsymbol{\beta} \quad$ Strain-displacement matrix

$\boldsymbol{\delta} \quad$ Nodal displacements along degrees of freedom

$\boldsymbol{\delta}_{a b, l} \quad$ Nodal displacements expressed in local frame

$\boldsymbol{\delta}_{a b, G} \quad$ Nodal displacements expressed in globally-aligned nodal frames

$\boldsymbol{\delta}_{n} \quad$ Nodal displacements expressed in globally-aligned nodal frames for all nodes

$\Delta_{d} \quad$ External damper compressed length

$\Delta_{d, 0} \quad$ External damper uncompressed length

$\Delta_{f} \quad$ Friction micro displacement 
$\Delta_{f, \max } \quad$ Maximum allowable friction micro-displacement

$\Delta_{p} \quad$ Surface penetration depth

$\Delta t \quad$ Current solution step successfully propagated time

$\Delta t_{\text {sol }} \quad$ Default solution propagation time-step length

$\Delta t_{e} \quad$ Solution propagation time-step length where event is detected

$\dot{\Delta}_{d} \quad$ External damper rate of compression

$\dot{\Delta}_{f} \quad$ Friction rate of micro-displacement

$\dot{\Delta}_{p} \quad$ Relative surface penetration velocity

$\boldsymbol{\epsilon} \quad$ Finite element material property matrix

$\gamma \quad$ ratio of specific heats

$\mu_{d} \quad$ Dynamic friction coefficient

$\mu_{s} \quad$ Static friction coefficient

$\omega_{1} \quad$ Proportional damping lower bound frequency

$\omega_{2} \quad$ Proportional damping upper bound frequency

$\phi_{h l} \quad$ Flow angle relative to rotor disc plane

$\psi \quad$ Rotor blade azimuth

$\psi_{l} \quad l$ th rotor blade sector azimuth

$\psi_{\max } \quad$ Azimuth of maximum cyclical pitch setting

$\rho \quad$ Finite element density

$\rho_{\text {air }} \quad$ Density of air

$\theta_{a} \quad$ Angular displacement of node $a$ for finite element spanning from node $a$ to node $b$

$\theta_{b} \quad$ Angular displacement of node $b$ for finite element spanning from node $a$ to node $b$

$\theta_{\text {coll }} \quad$ Rotor blade collective pitch setting

$\theta_{c y c_{l}} \quad$ Rotor blade cyclical pitch setting

$\theta_{c y c, \text { max }}$ Maximum rotor blade cyclical pitch setting

$\theta_{h l} \quad$ Rotor blade element pitch setting

$\theta_{t_{h}} \quad$ Rotor blade twist angle

$\Theta_{a b} \quad$ Dynamic finite element orientation relative to the global finite element frame

$\xi_{1} \quad$ Proportional damping lower bound damping ratio

$\xi_{2} \quad$ Proportional damping upper bound damping ratio 


\section{Acronyms}

\begin{tabular}{|c|c|}
\hline ASIST & Aircraft Ship Integrated Secure and Traverse \\
\hline ASV & Anti-surface vessel \\
\hline ASW & Anti-submarine warfare \\
\hline $\mathrm{BDF}$ & Back-differentiation \\
\hline BEM & Blade element model \\
\hline BEMT & Blade element momentum theory \\
\hline $\mathrm{BET}$ & Blade element theory \\
\hline $\mathrm{BVH}$ & Bounding volume hierarchy \\
\hline CFD & Computational fluid dynamics \\
\hline $\mathrm{CoG}$ & Centre of gravity \\
\hline $\mathrm{CPU}$ & Central processing unit \\
\hline CSM & Contact surface matrix \\
\hline DFE & Dynamic finite element \\
\hline DFMEA & Design failure mode and effect analysis \\
\hline DLSODAR & $\begin{array}{l}\text { Double-precision Livermore solver for ordinary differential equations } \\
\text { with automatic solver switching and root finding }\end{array}$ \\
\hline DLTV & Dead-load test vehicle \\
\hline DoF & Degree-of-freedom \\
\hline DRDC & Defence Research and Development Canada \\
\hline $\mathrm{OCP}$ & Object contact point \\
\hline ODE & Ordinary differential equation \\
\hline PSM & Penetration state matrix \\
\hline $\mathrm{PSCM}$ & Penetration state change matrix \\
\hline $\mathrm{RBE}$ & Rotor blade element \\
\hline SAR & Search and rescue \\
\hline SAT & Separation axis theorem \\
\hline
\end{tabular}




$\begin{array}{ll}\text { SPR } & \text { Spatial partitioning representation } \\ \text { SRAMSS } & \text { Skid-equipped Rotary-wing Aircraft Manoeuvring and Securing Sim- } \\ & \text { ulation } \\ \text { SSMASH } & \text { Spacial Securing and Manoeuvring Analysis for Shipboard Heli- } \\ & \text { copters } \\ \text { RAO } & \text { Response amplitude operator } \\ \text { RAST } & \text { Recovery Assist, Secure and Traverse } \\ \text { RHR } & \text { Right-hand rule } \\ \text { RSD } & \text { Rapid securing device } \\ \text { TC-ASIST } & \text { Twin-claw Aircraft Ship Integrated Secure and Traverse } \\ \text { UAS } & \text { Unmanned aircraft system }\end{array}$




\section{Chapter 1}

\section{Introduction}

The mobility of helicopters has been recognized and leveraged for maritime operations around the world, for the past half-century. Helicopters have seen a wide variety of roles including increasing ship line-of-sight and area-of-effect, search and rescue (SAR), maritime work-site transportation, anti-submarine warfare (ASW), and anti surface vessel (ASV) strike to name a few [1]. In mission-critical situations, ship-based helicopters are required to operate in severe weather conditions. These conditions can be hazardous to both air crew, deck crew, and the equipment itself.

Maritime shipboard operations in severe weather is an ideal example of a task considered hazardous to humans for which Unmanned Aircraft Systems (UAS) offer a widely-recognized beneficial alternative. Their capacity to operate in high-risk environments to humans, their preclusion of human error during operations, and progressively-declining operating costs are some of the motivations driving the increasing use of UASs. To this point, UAS usage has already proliferated into shipboard operations. For example: the U.S. Navy's use of the Northrop Grumman MQ-8B Fire Scout as a ship-based UAS system [9]; the Royal Canadian Navy has investigated the use of Armed Drones [10]; and QuinetiQ will be providing the Royal Canadian Navy with UAS based on the Skeldar V-200 UAS [11].

Whether a piloted helicopter or UAS, rotary-wing aircraft require assistive securing and manoeuvring devices to operate in challenging maritime conditions. For conventional maritime helicopters equipped with compliant landing gear and grippy tires, such as the SH-60 Seahawk shown in Figure 1.1, there exists assistive landing, securing, and manoeuvring devices for shipboard operations. Accordingly, simulation software specifically targeted at studying the dynamics of shipboard operations for 


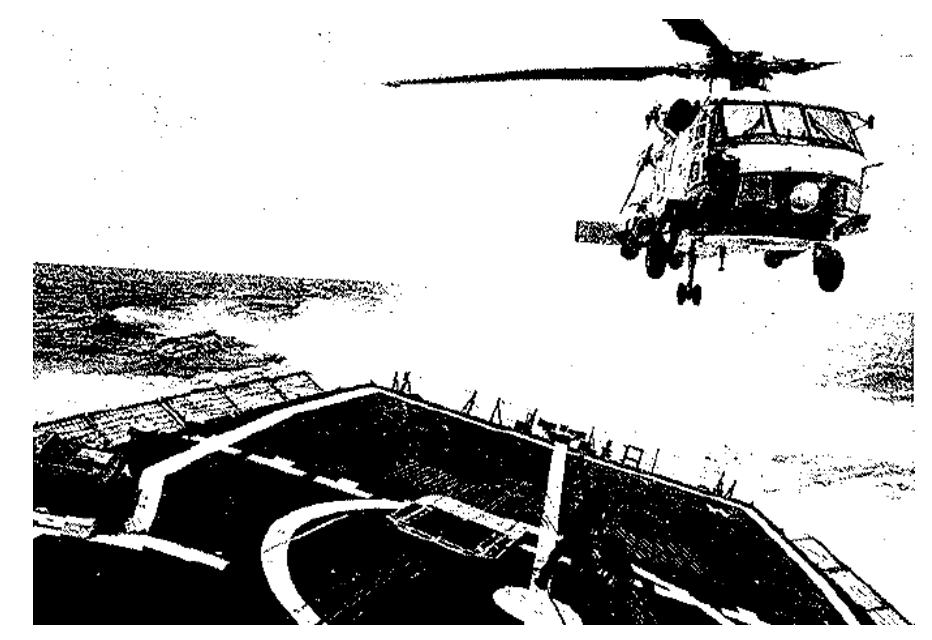

Figure 1.1: SH-60 Seahawk hovering over ship deck landing zone equipped with LAMPS III RAST system [1].

these types of helicopters is available.

The following sections present a literature review of a notable shipboard securing and manoeuvring systems and dynamic shipboard models. They will reveal the limitations in devices and associated dynamic models as they relate to UASs and other skid-equipped aircraft embarking on shipboard operations.

\subsection{Shipboard Securing and Manoeuvring Systems}

Transient ship motion in severe weather often exceeds the aircraft's capabilities to precisely station-keep above the ship deck while in flight, and remain stationary while on deck. In order to aid aircraft landing, securing, and on-deck manoeuvring, various assistive systems have been used to a great degree of success. Notable systems used by navies around the world include three Indal Technologies Inc. devices: Recovery Assist, Secure and Traverse (RAST), Aircraft Ship Integrated Secure and Traverse (ASIST), and the Twin-claw ASIST (TC-ASIST) $[1,2]$. Although the three systems utilize a Rapid Securing Device (RSD) on rails integrated into the ship deck to secure a probe fixed to the aircraft, they vary in their modes of operation.

First, the RAST system employs a recovery assist haul-down cable under constant tension to guide the aircraft towards the ship deck and the RSD probe trap, as shown in Figures 1.1 and in the technical drawing of Figure 1.2 [1]. Second, the ASIST systems are free landing systems where the RSD tracks the motion of the aircraft 

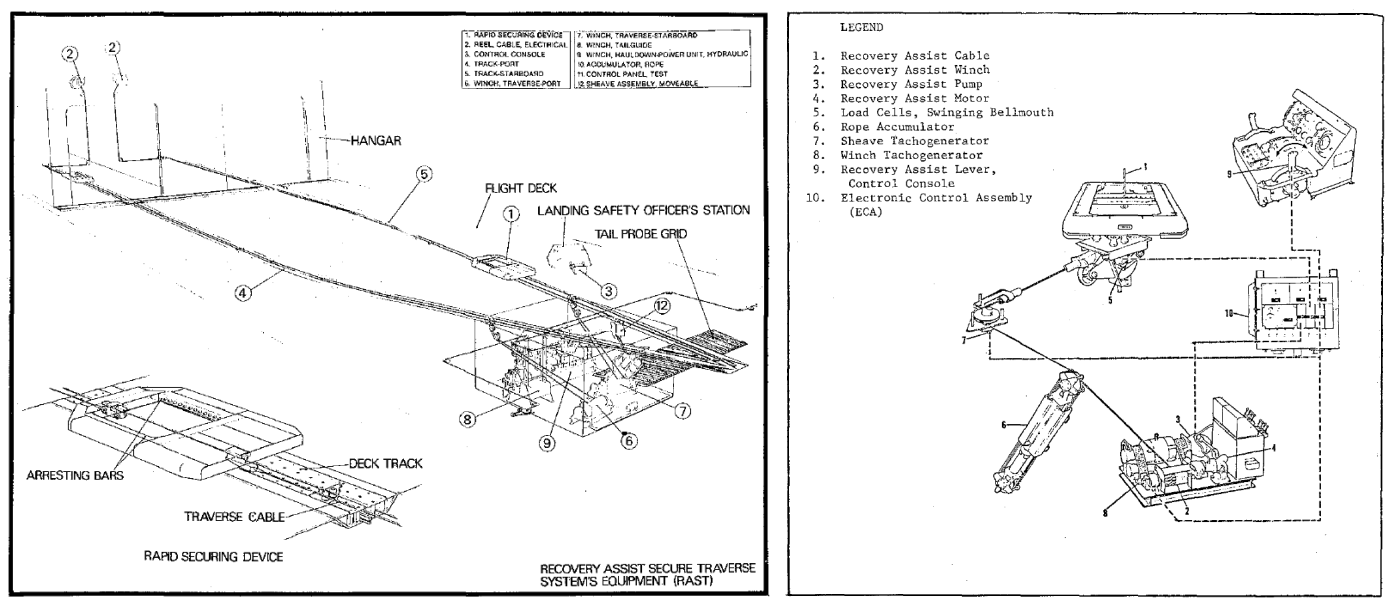

Figure 1.2: Recovery Assist, Secure and Traverse (RAST) system [1].

relative to the ship deck, and aligns to capture the securing probe upon aircraft touch-down on deck as shown in Figure 1.3 [2]. Lastly, TC-ASIST is an ASIST variant designed to work with probe-less aircraft [2]. In all cases, these devices are able to secure the aircraft to the ship. Notably, these devices are designed to work with aircraft using compliant landing gear with grippy tires, which enables the systems to both exploit available passive tire frictional forces in restraining the aircraft, and to manoeuvre the aircraft into shipborne hangars.

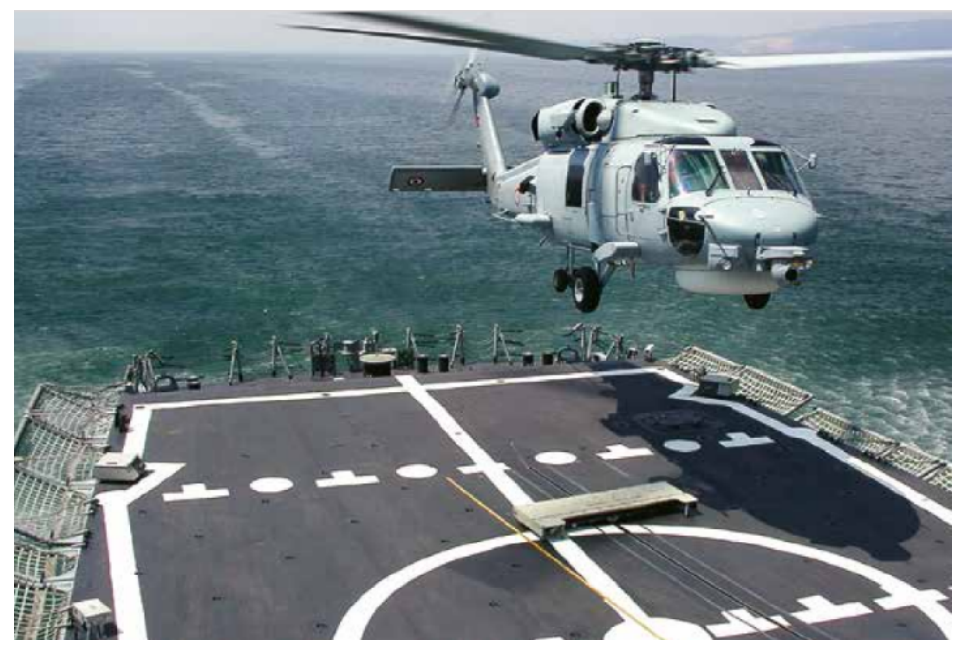

Figure 1.3: Aircraft Ship Integrated Secure and Traverse (ASIST) system [2].

Other ship deck securing devices exist. For example: the Cramms HLS Heligrid system uses a perforated disc fixed to the ship deck designed to receive a 'harpoon'style probe fixed to the aircraft which penetrates and locks to the disc [12]. This 
system can accommodate various styles of landing gear; however this system is limited by its inability to manoeuvre aircraft once they are secured. This presents a problem in more severe weather where the aircraft should be sheltered from the elements by a shipborne hangar. The aircraft would necessarily have to be transferred to another system such as the SAMAHE [13] for manoeuvring, and this transfer creates a securing vulnerability. In any case, being able to model the dynamic interaction between the ship and the aircraft is an essential tool in the design and operation of these securing systems and shipboard operation-capable aircraft.

\subsection{Shipboard Helicopter Modelling}

The dynamic interaction between the ship and aircraft, often referred to as 'dynamic interface analysis', is indispensable in determining factors including design loads, operational envelopes, performance, and operating procedures. Under the scope of dynamic interface analysis, there are many aspects to consider: ship deck motion resulting from hydrodynamic interactions between the ship hull and surrounding sea, aircraft landing, securing and manoeuvring dynamics, and aerodynamic effects resulting from ship superstructure air-wake and environmental factors.

\subsubsection{Ship Deck Motion}

Ship deck motion dominates the dynamics of a ship-embarked aircraft. There are commercially available packages such as SHIPMo3D which use a 3D panel method to determine transient ship motion, capable of outputting linear response amplitude operator (RAO) data for use in 3rd party software [14]. Indal Technologies Inc. has developed a fully 3D, non-linear, time-domain ship motion program called SPLAShMo to generate transient ship motion to be used with their dynamic interface analysis software Dynaface ${ }^{\circledR}[15,16]$. SPLAShMo's lack of linear simplifications makes it well suited to studying the motion of smaller naval vessels operating in higher sea states, which represent a significant subset of vessels undergoing shipboard rotarywing aircraft operations [15].

Other ship motion software packages include strip theory-based ShipmoPC, a derivative of SHIPMO 7 developed at Defence Research and Development Canada (DRDC) [17], which can account for hydrodynamic effects of hull appendages and output RAOs. Additionally, FREDYN is a blended time-domain method package 


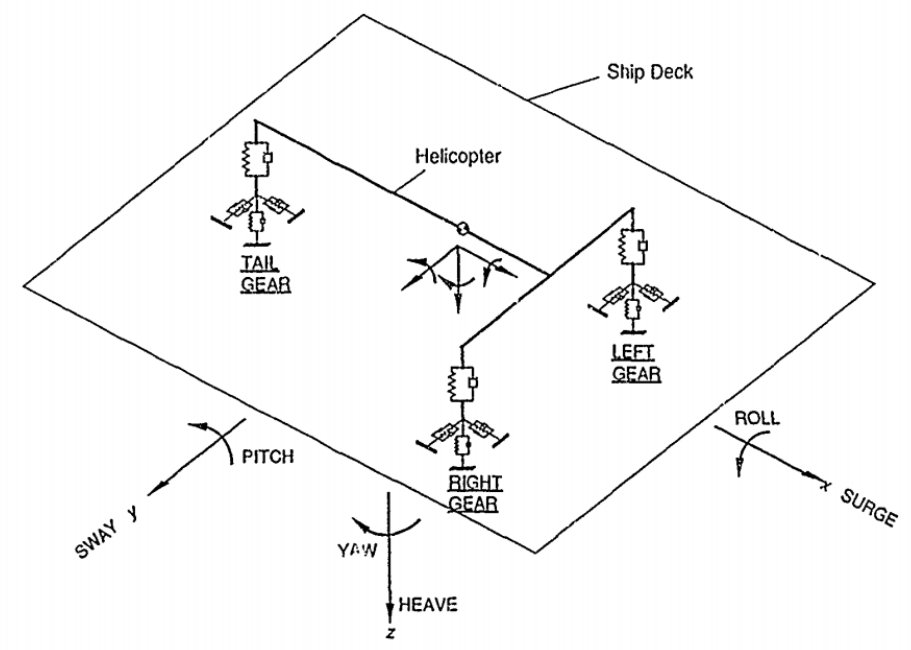

Figure 1.4: System description of Blackwell and Feik [3].

aimed at ship manoeuvring in calm waters [18].

\subsubsection{Securing and Manoeuvring Models}

As a culmination of many disciplines of engineering, dynamic interface analysis has been widely researched. Early research into dynamic interface modelling from Blackwell and Feik [3] characterized a single rigid body aircraft on an arbitrarily moving ship deck with two-stage non-linear damped spring oleo representation, and massless spring tire representation as shown in Figure 1.4. The work was limited to studying ship deck motion response, excluding aerodynamic, securing, and handling forces.

A refined simulation package named Dynaface ${ }^{\circledR}$ was developed by Langlois et al. [16] at Indal Technologies Inc. Also characterized as a single rigid body, the simulated aircraft is acted on by dynamic effects generated by ship motion, aerodynamics, landing gear suspension, tires, securing devices, and lashing cables. However, it is not capable of simulating shipboard transient manoeuvring.

Addressing embarked aircraft manoeuvring, Linn developed simulation software named Heliman [5] for the purpose of analyzing embarked helicopter handling and manoeuvring. Limited to the planar case without ship motion as shown in Figure 1.6, prescribed probe motion enabled the evaluation of aircraft planar translation and yaw (including castor steering) response. The lack of ship motion response analysis in $\mathrm{He}$ liman was addressed by an experimental motion platform developed by Feldman [19], 


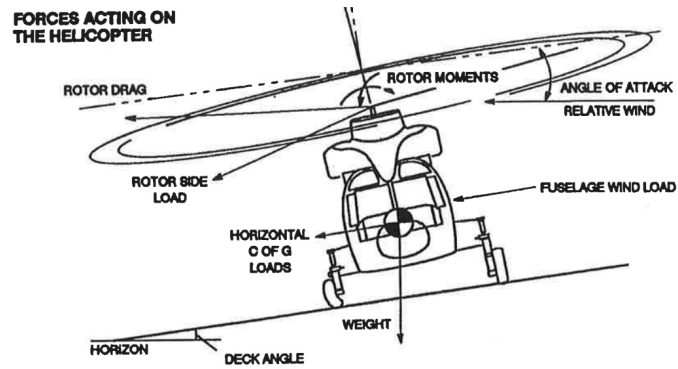

Figure 1.5: Force-generating elements included in Dynaface ${ }^{\circledR}[4]$.

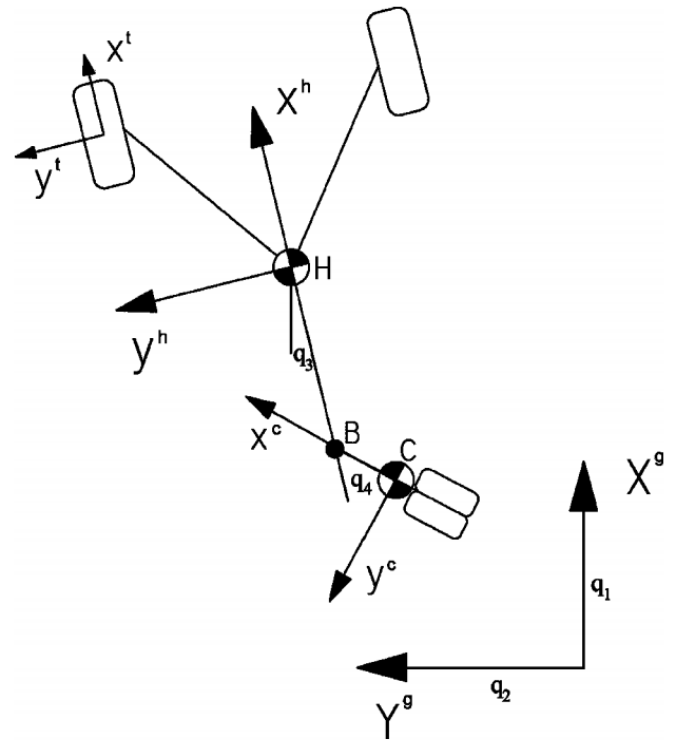

Figure 1.6: System description of Heliman [5].

capable of simulating combinations of surge, sway, heave, roll, and pitch motions. It included a 2 degree-of-freedom (DoF) scale model of Indal's ASIST system, and a scale Dead-Load Test Vehicle (DLTV). Experimental results highlighted the additive effects of the RSD manoeuvres and ship deck motion in securing forces experienced by the DLTV. Aerodynamic effects were not considered. It also concluded that autonomous embarked aircraft manoeuvring is possible. Subsequently Feldman et al. developed and implemented an autonomous straightening and traversing procedure [20].

Building on the efforts made by Langlois et al., Linn, and Feldman, the SSMASH (Spacial Securing and Manoeuvring Analysis for Shipboard Helicopters) was developed by Léveillé [21]. It provides complete analysis capabilities for the analysis of on-deck helicopter/ship dynamic interface. A fully-spacial aircraft model with bodies representing the aircraft and its landing gear, coupled with 6-DoF ship motion, and a 5-DoF tire model allow complete simulation of securing and manoeuvring of embarked aircraft under full ship motion conditions. However, it does not include the Rotor thrust and induced rotor forces characterized by Dynaface ${ }^{\circledR}[22]$.

Section 1.1 has described shipboard securing and manoeuvring systems, and the modelling thereof, for rotary wing aircraft which employ compliant landing gear equipped with grippy tires. As with helicopters, managing ship deck and aircraft dynamics for UASs is critical. However, for UASs, aerodynamic forces acting on 


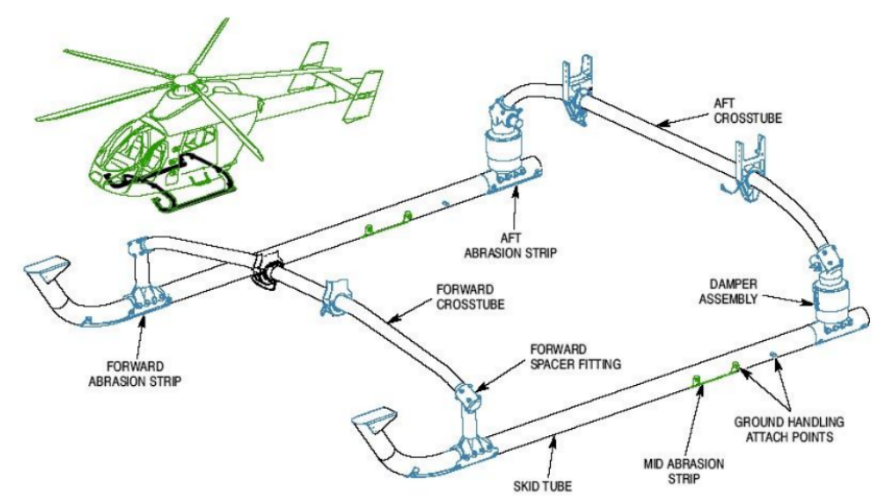

Figure 1.7: Skid-type landing gear description [6].

the UAS become even more important due to the higher thrust to weight ratio of typical UASs compared to helicopters. Furthermore, UASs often use less compliant 'skid-type' landing gear which constrain mechanical securing and manoeuvring options, and present more complex ship-helicopter interface behaviours which must be characterized.

\subsubsection{Skid-type Landing Gear Models}

Skid-type landing gear are lightweight alternatives in comparison to conventional wheeled landing gear. As shown in Figure 1.7, skid-type landing gear are composed of structural tubes assembled to form two main parallel skid-tubes for contact with the ground, which are attached to the aircraft by cross-tubes. Analyzing skid-equipped aircraft undergoing shipboard manoeuvres in hazardous weather is critical to the safety of deck crews and equipment.

With the increasing use of UASs in shipboard operations, Indal Technologies Inc. recognized both the proliferation of UAS usage in [23] and the trend of equipping UAS with skid type landing gear in [6]. As a result, an extension for Dynaface ${ }^{\circledR}$ was created with two skid landing gear models; one using a fast linear stiffness method, and the other using a non-linear finite element structural modelling approach [6]. The extension allowed for the dynamic interface analysis of an embarked skid-equipped aircraft acted on by aerodynamics, securing mechanisms, and ship deck motion. However, as previously stated, Dynaface ${ }^{\circledR}$ is unable to simulate shipboard manoeuvring.

The author is unaware of any other simulation software that specifically addresses 
skid-type landing gear within the framework of dynamic interface analysis.

\subsubsection{Aerodynamic Ship-helicopter Interactions}

Beyond the significant effects that ship motion have on embarked helicopters, aerodynamic effects are equally important. A wide range of phenomena have been studied. Lee and Zan studied the ship's airwake effects on rotorless aircraft fuselage loads [24]. Zan conducted sub-scale experiments to determine rotor thrust in ship airwakes, and the influence of ship geometry and wind speed direction [25]. Alpman et al. combined computational fluid dynamics (CFD) and a flight dynamics simulation model to study the dynamic interface of UH-60A helicopter hovering behind an aircraft hangar and the same helicopter approaching an LHA-class ship [26]. Wall et al. used an advancing Fourier series method for correlated turbulence modelling for a point moving through space [27]. In this case, to study a point on a helicopter blade, which was part of a larger scope modelling rotor blade sailing in unsteady airwakes by Wall et al. [28]. Even in the absence of ship airwakes, Khouli et al. showed for aeroelastic models that combinations of ship roll/pitch frequencies, and rotor disc frequencies could cause rotor tip displacements outside acceptable operating ranges [29].

A comprehensive real-time simulation package named RotorLib FDM was created by RTDdynamics [30]. It includes a Blade Element Theory (BET) model which captures compressibility effects, ground effects, and autorotation. Moreover, the aircraft models are highly configurable, and the full flight regime of the aircraft can be modelled, including landing and take-off from moving surfaces.

\subsubsection{Rotary-wing Aerodynamics Modelling}

Section 1.2.4 highlights the breadth of effects that aerodynamic phenomena induce on rotary-wing aircraft near and embarked on ships. The typically higher thrust-toweight ratio of UASs increases the effects of these aerodynamic phenomena. Both Dynaface ${ }^{\circledR}$ and SSMASH make efforts to characterize aerodynamic drag forces on the aircraft fuselage based on the relative aircraft velocity with respect to the apparent wind velocity and direction. Dynaface ${ }^{\circledR}$ also models rotor thrust using a time-dependent profile of rotor thrust. The thrust vector is transformed to equipollent forces and moments on the aircraft. At low rotor disc RPM it models induced rotor forces on the aircraft by double interpolation of the force components against 
rotor disc's angle of attack and wind direction [22]. Neither simulation package considers phenomena such as blade sailing or ship airwake in the aerodynamic effects on the aircraft. Moreover, the lookup tables used in the double interpolation for induced forces used by Dynaface ${ }^{\circledR}$ are typically provided and protected by the manufacturer.

Stepniewski [43] presents basic theories for approaches to modelling rotor disc thrust generation. Models derived from Blade Element Theory (BET) and Blade Element Momentum Theory (BEMT) provide adequate fidelity in rotor performance without computational costs typically associated with CFD analysis [44], or requiring manufacturer-provided experimental or computational data. These models are highly adaptable for including many aerodynamic phenomena and thereby increasing fidelity as required. Blade element models discretizes the rotor blade into rotor blade elements which are assigned individual aerodynamic parameters and properties required for the application of basic lifting theories. Since the individual elements are assigned unique properties, more complex blade profiles can be approximated without the need for computationally expensive CFD analysis.

Having reviewed a number of applicable shipboard helicopter models and aerodynamic models, there is limited consideration made for the complex transient contact states between the aircraft and ship deck. For UAS typically equipped with skid-type landing gear, the rigidity of the landing gear creates complex and evolving contact states. As a result, a more comprehensive contact model should be considered. The following sections survey contact modelling and friction modelling.

\subsection{Contact Modelling}

The trend of UASs being equipped with skid-type landing gear, which are less compliant than conventional landing gear, realises an important consideration in the dynamic interface modelling of these types of aircraft. The rigidity of skid-type landing gear leads to complex, intermittent contact states. This leads to the requirement for a more refined contact model for dynamic interface analysis than those included in the securing and handling models presented in Section 1.2.2.

Contact modelling can be divided into two parts. First, the detection of collisions between objects studied can be separated into 'broad-phase' and 'narrow-phase' contact determination algorithms [31]. Broad-phase is a coarse search for pairs of objects with the potential for collision whereas narrow-phase is the direct evaluation of 
contact. For larger systems, collision detection algorithms typically start with broadphase detection then pass to narrow-phase contact evaluation. Collision detection can be further classified into 'Spatial Partitioning Representations' (SPR) and 'Bounding Volume Hierarchies' (BVH) [32]. The SPR is a discrete representation the simulation environment through the use of cells where pairs of objects occupying the same cells are evaluated for contact, and is well suited to broad-phase collision detection. Bounding volume hierarchies assign a volume which bounds the object evaluated for contact. these bounding volumes can be simple shapes which greatly increases contact determination efficiency. The Separation Axis Theorem (SAT) collision detection algorithm [33], based on the BVH 'oriented bounding box' [34] algorithm, lends itself well to the study of dynamics since the bounding volumes can exactly take the shape of the polygons representing objects.

Second, once collision is detected, the impact must be treated. Gilardi and Sharf [35] survey different methods for contact dynamics modelling. Many models exist, including discrete, continuous, Lagrangian multiplier, Hertzian models, etc. Notably, continuous closed-form models such as the spring-dashpot, Hertz, and non-linear damping models are easily integrated with the SAT algorithm to provide appropriately-oriented dynamic response to collision. These models consider collision restitution to maintain impenetrability of the colliding bodies. Friction modelling must also be present for the handling of prolonged contact between bodies.

\subsection{Friction Modelling}

Friction forces are an important component in generating an appropriately-oriented dynamic response to collision. In the securing and handling models of Section 1.2.2, contact friction modelling has fallen under the scope of tire models of conventional landing gear. They rely on a frictional slider implementing a virtual spring-damper model which saturates to the Coulomb friction value. For skid-type landing gear with hard contact surfaces, the transition between sticking and slipping is rapid due to less compliant materials when compared to the soft rubber tires of wheeled landing gear. Therefore a friction model which can accurately capture more complex phenomena is required.

Lampert et al. [36], Liu et al. [37], Marques et al. [38], and Pennestrì et al. [39] generally conclude that the LuGre friction model [40], an improvement of the Dahl 
model [41], captures frictional phenomena accurately. The LuGre model's use of 'bristles' is analogous to the frictional-slider models of current securing and handling software packages. It is able to model static, dynamic, and viscous friction, as well as pre-sliding micro displacements through the use of a Stribeck curve [40].

Swevers et al. [42] discuss the issues present in the hysteresis curves for the LuGre model in the pre-sliding regime. They propose the Leuven model, which implements a Maxwell-slip model into a hysteretic function for the pre-sliding regime, but is otherwise identical to the LuGre model. Lampert et al. note that the Leuven model agrees best with experimental results. Despite its accuracy, the hysteretic function of the Leuven model is more computationally expensive. It requires the addition of another state variable to reset the hysteretic function if sliding motion is achieved. Furthermore, the precision required by control systems in which these models are typically used is not as significant at the scale of dynamic interface analysis.

\subsection{Thesis Objectives}

As reviewed above, ship-helicopter interface dynamics have been extensively studied through many avenues. It has exposed the limitations in modelling interface dynamics and securing devices for skid-equipped UASs. With the proliferation of UASs in shipboard operations, the necessity to study their interface dynamics becomes apparent.

As with helicopters, managing ship deck and aircraft dynamics for UASs is critical. However, aerodynamic forces acting on the UAS become even more important due to the UAS's higher thrust-to-weight ratio compared to helicopters. Furthermore, UASs often use less-compliant skid-type landing gear unlike conventional maritime helicopters that most commonly use compliant suspensions and grippy tires. These typical UAS characteristics constrain mechanical securing and traversing options, and present more complex ship-helicopter interface behaviours which must be characterized. Therefore a simulation package which can accurately capture the complex interface dynamics of stiff landing gear aircraft is necessary for the investigation of securing requirements and ensuring expected performance of all components in shipboard operations. Table 1.1 presents a summary of the existing models introduced in Section 1.2.2. 
Table 1.1: Shipboard securing and manoeuvring modelling summary

\begin{tabular}{|c|c|c|c|c|c|c|c|}
\hline Model & & Wheel & Skid & Securing & Manoeuv. & $\begin{array}{l}\text { Ship } \\
\text { Motion }\end{array}$ & Aero. \\
\hline Blackwell-Feik & $3 \mathrm{D}$ & $\checkmark$ & & & & $\checkmark$ & \\
\hline Heliman & $2 \mathrm{D}$ & $\checkmark$ & & $\checkmark$ & $\checkmark$ & & \\
\hline Feldman & $3 \mathrm{D}$ & $\checkmark$ & & $\checkmark$ & $\checkmark$ & $\checkmark$ & \\
\hline Dynaface ${ }^{\circledR}$ & $3 \mathrm{D}$ & $\checkmark$ & & $\checkmark$ & & $\checkmark$ & $\checkmark$ \\
\hline Dynaface ${ }^{\circledR}$ Skid & $3 \mathrm{D}$ & & $\checkmark$ & $\checkmark$ & & $\checkmark$ & $\checkmark$ \\
\hline RotorLib & $3 \mathrm{D}$ & $\checkmark$ & $\checkmark$ & & & $\checkmark$ & $\checkmark$ \\
\hline SSMASH & $3 \mathrm{D}$ & $\checkmark$ & & $\checkmark$ & $\checkmark$ & $\checkmark$ & $\checkmark^{1}$ \\
\hline This Work & $2 \mathrm{D}^{2}$ & $\checkmark^{3}$ & $\checkmark$ & $\checkmark$ & $\checkmark$ & $\checkmark$ & $\checkmark$ \\
\hline
\end{tabular}

1 Only aerodynamic body drag forces.

${ }^{2}$ This work develops and verifies the planar case, with the intent of expansion into $3 D$

${ }^{3}$ Not within the scope of this work, but feasible.

Table 1.1 shows significant progress has been made with respect to the dynamic interface analysis of wheeled-landing gear helicopters. The proliferation of UAS in maritime shipboard operations and lack of availability of securing systems which can manoeuvre secured skid-equipped aircraft reveal a gap in the capabilities in the current state of dynamic interface analysis. The objective of this work is to the fill the gap revealed in Table 1.1 by developing the planar (2D) case of a fully-spacial simulation package specifically aimed at skid-equipped aircraft. To achieve completeness of the objective, the simulation package should:

- Identify conceptual design requirements for a shipboard securing and manoeuvring system for skid-equipped rotary-wing aircraft;

- Develop fully 2D on-and off-deck modelling of aircraft dynamics, with dynamic system derivation and methodology appropriate for expansion to 3D;

- Simulate the complete recovery flight, securing, manoeuvring, and launch phases of shipboard helicopter operations;

- Combine rigid and flexible body dynamics into a single dynamic system;

- Model infinitely-configurable skid-type and other stiff landing gear;

- Model a comprehensive set of aerodynamic phenomena present in recoveryflight, embarked, and take-off phases; 
- Incorporate full-DoF ship motion, where the planar case includes heave, sway, and roll;

- Generate appropriately-oriented dynamic response at detection of contact;

- Present a suitable ship deck securing and manoeuvring system proof-of-concept.

In effect, this work represents the planar case of the combined capabilities of Dynaface ${ }^{\circledR}$ with the skid landing gear extension, SSMASH's fully spatial securing and manoeuvring capabilities, and includes more detailed contact mechanics and aerodynamic models. The scope of this work is to develop and verify the planar dynamic system formulation and simulation architecture for the dynamic interface analysis of skid-equipped rotary-wing aircraft such that an acceptable methodology is developed for the creation of a fully-spatial package. As it relates to the field dynamic interface analysis, this work primarily expands simulation capabilities to include the securing and manoeuvring of embarked skid-equipped aircraft. Moreover it contributes the system formulation necessary to simulate the rigid-flexible body behaviour of skid-equipped aircraft. Consequently the work also contributes a general appropriately-oriented contact dynamic algorithm as a result of characterizing the complex contact behaviour of compliant bodies which is not limited to the modelling of skid-type landing gear. An additional contribution evolves from the proposed conceptual design of a securing system which can also manoeuvre embarked skidequipped aircraft.

Future work beyond the established scope will be performed to expand this work into a fully-spatial simulation package based on the verified methodology developed in this work.

\subsection{Thesis Overview}

This chapter has presented the current progression of shipboard helicopter operations, a significant cross-section of available shipboard securing and manoeuvring systems, the state-of-the-art in dynamic interface analysis software, and identified a gap in the aforementioned that this work aims to fill with respect to skid-equipped rotary-wing aircraft. Going forward, this work describes the preliminary requirements and design of the securing and manoeuvring device, mathematical definitions and methods, and dynamic system definition in Chapter 2. Following this, Chapter 3 presents the 
derivations of the kinematic equations required for the dynamical system. Chapter 4 presents the derivations of forces generated by the landing gear, contact dynamics, aerodynamics, and other dynamic models. The kinematics of Chapter 3 and dynamics of Chapter 4 are assembled into a linear dynamic system in Chapter 5. Verification, and preliminary validation of the final dynamic system formulation and models implemented are discussed in Chapter 6. Conclusions of the completed work are drawn, and recommendations for future work are made in Chapter 7 . 


\section{Chapter 2}

\section{System Description}

This work has developed a simulation package for the dynamic interface analysis of skid-equipped rotary-wing aircraft named SRAMSS (Skid-equipped Rotary-wing Manoeuvring and Securing Simulation). This chapter presents the requirements and definitions necessary to generate the dynamic system in SRAMSS. Section 2.1 describes and defines a preliminary concept for a securing and manoeuvring device for skid-equipped aircraft as required for the SRAMSS simulation environment since embarked skid-equipped aircraft manoeuvring is a simulation requirement for SRAMSS. Section 2.2 covers mathematical notations, conventions, and the methods for dynamic equation generation and skid-type landing gear modelling. With the definitions formulated in the previous sections, Section 2.3 defines the generalized coordinates, generalized speeds, and the structure of frames of reference used for the derivation of the complete dynamic system.

\subsection{Securing and Manoeuvring System Concep- tual Design}

SRAMSS addresses the limitations of current dynamic interface analysis software as it pertains to the simulation of embarked manoeuvring of skid-equipped aircraft. Since the author has not found the existence of any available securing and manoeuvring systems for skid-equipped aircraft, such a system must be defined to guide the development of the simulation environment in SRAMSS. The rapid securing device design contributes to the thesis objectives of system conceptual design that enables embarked securing and manoeuvring, as described in Section 1.5. The general requirements for 
the securing system, and preliminary concept are described in the following sections.

\subsubsection{Securing and Manoeuvring Mechanism Requirements}

High-level requirements for the securing and manoeuvring concept are derived from two areas: existing securing and manoeuvring system capabilities, and UAS/skidequipped aircraft considerations. With respect to existing systems and literature [4], requirements include:

- Passive securing: a structural member attached to the aircraft is captured to secure the aircraft to the ship deck. Loads are transferred to the ship structure;

- Controlled yaw motion of aircraft relative to the ship deck for hangar alignment;

- Controlled longitudinal motion with lateral restraining for manoeuvres across the ship deck;

- Securing aircraft against ship motion in up to sea-state 6; and

- RSD which captures the aircraft fast enough to avoid unrestrained aircraft motion.

In considering the type of aircraft used, requirements include:

- Passive securing device completely contained on the ship due to limited UAS payloads and to limit reduction in UAS range;

- Device completely contained on the ship enables a universal design philosophy which can be sized and adapted to suit the majority of skid-equipped rotarywing aircraft;

- Manoeuvrable landing and securing platform due to immobile nature of secured skid-equipped aircraft; and

- Platform range of motion capable of correcting for expected landing dispersion.

\subsubsection{Skid-type Securing and Manoeuvring Concept}

Emerging from the requirements listed in Section 2.1.1, capabilities for the skid-type securing and manoeuvring device consist of:

- Device completely contained to the ship, with universal design capable of accommodating a variety of skid-equipped aircraft;

- Passive RSD capable of securing a skid-equipped aircraft in up to sea-state 6; 


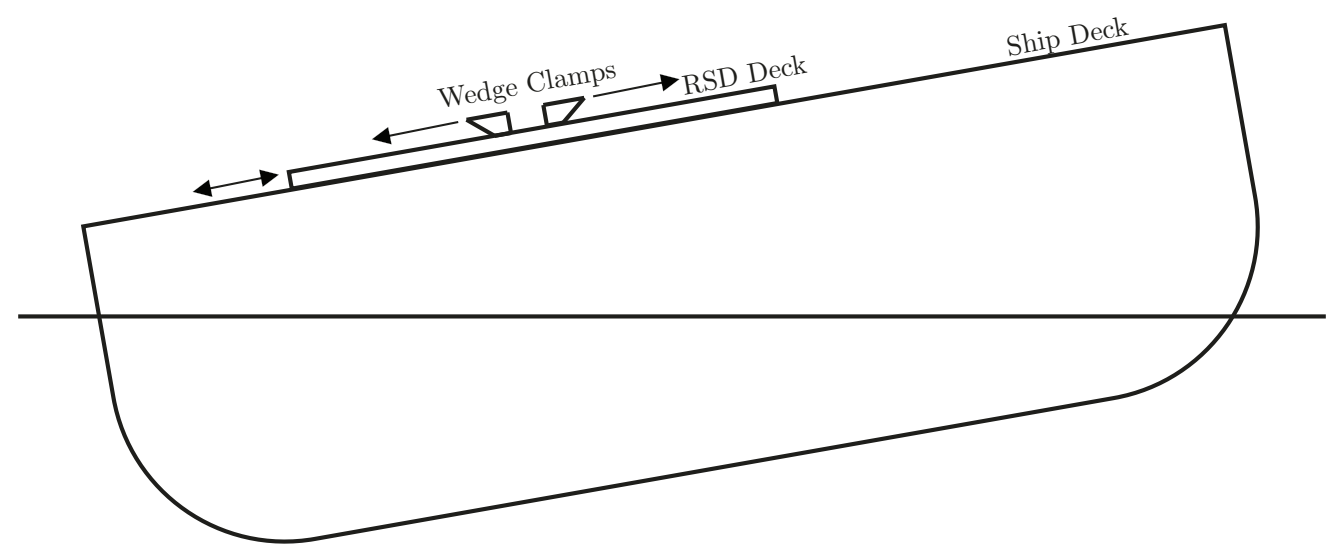

Figure 2.1: Planar representation of the skid-equipped securing and manoeuvring system.

- Landing platform with manoeuvring capabilities to move an embarked aircraft into a shipborne hangar;

To capture these capabilities, this work proposes a manoeuvrable platform with longitudinal, limited-transverse, and yaw DoFs, with wedge shaped clamps which move outwards in the transverse direction. Outward-moving clamps are expected to be less prone to causing aircraft roll-overs as the fulcrum created by a skid tube caught in the wedge is necessarily behind the aircraft's CoG under sliding motion. The platform should be able to interface with existing RAST and ASIST systems for expanded operational capabilities, though this aspect is not discussed further. For the planar case in this work, Figure 2.1 describes the proposed concept.

The planar case of the device includes only the transverse translation direction for the platform and wedge clamps. Aircraft securing with landing dispersion, transverse alignment to the hangar, and response to flight deck heave, sway, and roll are still observable in the planar case. Nevertheless, this description of the securing and manoeuvring system completes the physical description of the simulation environment for SRAMSS. Mathematical notations, methods, and system descriptions are now possible.

\subsection{Mathematical Conventions and Methods}

To derive the system and models present in SRAMSS, mathematical conventions, identities, and methods must be defined. 


\section{Vector Naming Conventions}

A consistent vector notation is used to represent vector quantities. Boldfaced variables represent vector quantities, unless enclosed by square brackets [ ] which represent matrices.

Vectors with physical interpretations such as kinematic and kinetic vectors, and unit vectors are accented by a right pointing arrow " $\rightarrow$ " and circumflex " " respectively. Kinematic vector quantities are used to represent displacements, velocities, and accelerations of bodies and points in space, while kinetic vectors represent forces and moments applied to bodies. Given the complexity of the developed system, a rigorous vector subscript and superscript notation is adpoted from Léveillé [45] due to similar system definition and for precise vector description.

\section{Kinematic Vectors}

For the kinematic vector ${ }_{d}^{c} \overrightarrow{\boldsymbol{a}}^{b}$ :

- Kinematic vector quantities are represented by lower-case bold characters.

- $\boldsymbol{a}$ is the kinematic vector quantity type. In this work, $\boldsymbol{a}$ takes the form of $\boldsymbol{r}$ for translational displacement, $\boldsymbol{v}$ for translational velocity, $\boldsymbol{a}$ for translational acceleration, $\boldsymbol{\theta}$ for angular displacement, $\boldsymbol{\omega}$ for angular velocity, $\boldsymbol{\alpha}$ for angular acceleration, and $\boldsymbol{g}$ for gravitational acceleration;

- $b$ is the body for which the vector quantity is described;

- $c$ is the body, or frame of reference relative to which the vector quantity is described;

- $d$ is the frame of reference in which the vector quantity is expressed.

- As such, the kinematic vector quantity ${ }_{d}^{c} \overrightarrow{\boldsymbol{r}}^{b}$ is defined to be the position of body $b$ with respect to $c$, expressed in frame of reference $d$.

\section{Kinetic Vectors}

For the kinetic vector ${ }_{h}^{g} \overrightarrow{\boldsymbol{e}}^{f}$ :

- Kinetic vector quantities are represented by upper-case bold characters.

- $\boldsymbol{e}$ is the kinetic vector quantity type. It takes the form of $\boldsymbol{F}$ for force vectors and $\boldsymbol{M}$ for moment vectors;

- $f$ is forcing element or body;

- $g$ is the body on which $f$ is acting; 
- $h$ is the frame of reference in which the kinetic vector quantity is expressed.

- As such, the kinetic vector quantity ${ }_{d}^{c} \overrightarrow{\boldsymbol{F}}^{b}$ is defined to be force of $b$ acting on $c$, expressed in frame of reference $d$.

This work presents the planar 2D case of the SRAMSS simulation environment. All translation vector quantities are in the form of $2 \times 1$ column vectors, where the first and second entries are the $x$ and $y$ axis vector components respectively. All angular vector quantities are in the form of $1 \mathrm{x} 1$ column vectors, where the entry represents the angular vector quantity about a $z$ axis obeying the Right-Hand-Rule (RHR) for $x-y-z$ axis orthogonality, and RHR for angular quantity direction about the $z$ axis, i.e. the $z$ axis projects outwards from the page, and the positive angular direction is counter-clockwise.

\section{Vector Quantity Time Derivatives}

The time differentiated vector quantity is denoted

$$
\frac{\mathrm{d}}{\mathrm{d} t}\left({ }_{d}^{c} \overrightarrow{\boldsymbol{a}}^{b}\right)={ }_{d}^{c} \dot{\overrightarrow{\boldsymbol{a}}}^{b}
$$

For this work, the differentiation is performed in the frame of reference of the differentiated vector, and is referred to as the locally evaluated time derivative. This is significant, as obtaining real accelerations for the dynamic system requires the local time differentiations be evaluated in an inertial frame of reference. Thus the relative position vector quantities, defining the position of a body through multiple frames of reference, must all be expressed with respect to the inertial frame before differentiation can take place.

\section{Cross Product Identity}

Cross products appear in many instances of kinematic and dynamic derivations, for example: in locally evaluated time derivatives, and moment evaluations due to translational forces. Per Nikravesh [46], cross product evaluation for 3x1 vectors can be expressed as the matrix multiplication:

$$
\vec{a} \times \vec{b}=\widetilde{[\vec{a}]}] \vec{b}=-\widetilde{\vec{b}]} \vec{a}
$$


where, for vector $\overrightarrow{\boldsymbol{a}}$ with elements $a_{1}, a_{2}$, and $a_{3}$ along the $x, y$, and $z$ axes respectively, $\widetilde{[\overrightarrow{\boldsymbol{a}}]}$ is the skew-symmetric matrix of $\overrightarrow{\boldsymbol{a}}$, and takes the form:

$$
\widetilde{[\vec{a}]}=\left[\begin{array}{ccc}
0 & -a_{3} & a_{2} \\
a_{3} & 0 & -a_{1} \\
-a_{2} & a_{1} & 0
\end{array}\right]
$$

For the planar case, evaluating cross products of kinematic vectors, Equation 2.2 reduces to:

$$
\overrightarrow{\boldsymbol{\omega}} \times \overrightarrow{\boldsymbol{r}}=\widetilde{[\overrightarrow{\boldsymbol{\omega}}]} \overrightarrow{\boldsymbol{r}}=\left[\begin{array}{cc}
0 & -\omega \\
\omega & 0
\end{array}\right]\left\{\begin{array}{l}
r_{x} \\
r_{y}
\end{array}\right\}=\left\{\begin{array}{l}
v_{x} \\
v_{y}
\end{array}\right\}
$$

and for evaluating cross products of kinetic vectors, Equation 2.2 reduces to:

$$
\overrightarrow{\boldsymbol{r}} \times \overrightarrow{\boldsymbol{F}}=\widetilde{[\overrightarrow{\boldsymbol{r}}]} \overrightarrow{\boldsymbol{F}}=\left[\begin{array}{ll}
-r_{y} & r_{x}
\end{array}\right]\left\{\begin{array}{l}
F_{x} \\
F_{y}
\end{array}\right\}=M_{z}
$$

\section{Rotational Transformation Matrix}

Transformation matrices are used in the kinematic and dynamic derivations to transform the expression of a vector quantity from one frame of reference to another. In the planar case, only rotations about the $z$ axis can occur. The transformation $[\boldsymbol{T}]$ from rotated frame of reference $b$ to non-rotated frame of reference $a$ using an Euler angle rotation matrix about the $z$ axis is given [46]:

$$
\left[\boldsymbol{T}_{a \leftarrow b}\right]=\left[\begin{array}{ccc}
\cos (\theta) & -\sin (\theta) & 0 \\
\sin (\theta) & \cos (\theta) & 0 \\
0 & 0 & 1
\end{array}\right]
$$

For this work, all frame of reference $z$ axes are aligned in the same direction, and obey the RHR for axis orthogonality, therefore by inspection the transformation matrix presented in Equation 2.6 can be written

$$
\left[\boldsymbol{T}_{a \leftarrow b}\right]=\left[\begin{array}{ccc}
\cos \left({ }_{a}^{a} \overrightarrow{\boldsymbol{\theta}}^{b}\right) & -\sin \left({ }_{a}^{a} \overrightarrow{\boldsymbol{\theta}}^{b}\right) & 0 \\
\sin \left({ }_{a}^{a} \overrightarrow{\boldsymbol{\theta}}^{b}\right) & \cos \left({ }_{a}^{a} \overrightarrow{\boldsymbol{\theta}}^{b}\right) & 0 \\
0 & 0 & 1
\end{array}\right]=\left[\begin{array}{ccc}
\cos \left({ }_{b}^{a} \overrightarrow{\boldsymbol{\theta}}^{b}\right) & -\sin \left({ }_{b}^{a} \overrightarrow{\boldsymbol{\theta}}^{b}\right) & 0 \\
\sin \left({ }_{b}^{a} \overrightarrow{\boldsymbol{\theta}}^{b}\right) & \cos \left({ }_{b}^{a} \overrightarrow{\boldsymbol{\theta}}^{b}\right) & 0 \\
0 & 0 & 1
\end{array}\right]
$$

From this identity, the transformation matrix for $2 \times 1$ translational vectors is

$$
\left[\boldsymbol{T}_{a \leftarrow b}\right]=\left[\begin{array}{cc}
\cos \left({ }_{b}^{a} \overrightarrow{\boldsymbol{\theta}}^{b}\right) & -\sin \left({ }_{b}^{a} \overrightarrow{\boldsymbol{\theta}}^{b}\right) \\
\sin \left({ }_{b}^{a} \overrightarrow{\boldsymbol{\theta}}^{b}\right) & \cos \left({ }_{b}^{a} \overrightarrow{\boldsymbol{\theta}}^{b}\right)
\end{array}\right]
$$


and the transformation matrix for $1 \mathrm{x} 1$ angular vector quantities is unity. which agrees with the frame of reference interchangeability of the relative angles used in Equation 2.7 where the transformed vector is $\left\{\begin{array}{lll}x & y & \theta\end{array}\right.$.

For successive frame transformations, pre-multiplication of the consecutive transformation matrices in the chain of frames is required since consecutive frames are described with respect to the axes of the previous frame. For example, the transformation of rotated frame of reference $c$ to non-rotated frame of reference $a$, passing through rotated frame of reference $b$ is

$$
\left[\boldsymbol{T}_{a \leftarrow c}\right]=\left[\boldsymbol{T}_{a \leftarrow b}\right]\left[\boldsymbol{T}_{b \leftarrow c}\right]
$$

\section{Rotational Transformation Matrix Time Derivative Identity}

Time differentiation of transformation matrices occur during the differentiation of position vectors to velocity vectors then to acceleration vectors. As a result, the time derivative of a transformation matrix is:

$$
\begin{aligned}
& {\left[\dot{\boldsymbol{T}}_{a \leftarrow b}\right]=\frac{\mathrm{d}}{\mathrm{d} t}\left(\left[\boldsymbol{T}_{a \leftarrow b}\right]\right)} \\
& =\left[\begin{array}{cc}
-{ }_{b}^{a} \dot{\vec{\theta}}^{b} \sin \left({ }_{b}^{a} \overrightarrow{\boldsymbol{\theta}}^{b}\right) & -{ }_{b}^{a} \dot{\overrightarrow{\boldsymbol{\theta}^{b}}} \cos \left({ }_{b}^{a} \overrightarrow{\boldsymbol{\theta}}^{b}\right) \\
{ }_{b}^{a} \dot{\overrightarrow{\boldsymbol{\theta}}}^{b} \cos \left({ }_{b}^{a} \overrightarrow{\boldsymbol{\theta}}^{b}\right) & -{ }_{b}^{a} \dot{\overrightarrow{\boldsymbol{\theta}}}^{b} \sin \left({ }_{b}^{a} \overrightarrow{\boldsymbol{\theta}}^{b}\right)
\end{array}\right] \\
& =\left[\begin{array}{cc}
\cos \left({ }_{b}^{a} \overrightarrow{\boldsymbol{\theta}}^{b}\right) & -\sin \left({ }_{b}^{a} \overrightarrow{\boldsymbol{\theta}}^{b}\right) \\
\sin \left({ }_{b}^{a} \overrightarrow{\boldsymbol{\theta}}^{b}\right) & \cos \left({ }_{b}^{a} \overrightarrow{\boldsymbol{\theta}}^{b}\right)
\end{array}\right]\left[\begin{array}{cc}
0 & -{ }_{b}^{a} \overrightarrow{\boldsymbol{\omega}}^{b} \\
{ }_{b} \overrightarrow{\boldsymbol{\omega}}^{b} & 0
\end{array}\right] \\
& {\left[\dot{\boldsymbol{T}}_{a \leftarrow b}\right]=\left[\boldsymbol{T}_{a \leftarrow b}\right] \widetilde{\left[\vec{\omega}_{b}^{a} \overrightarrow{\boldsymbol{\omega}}^{b}\right]}}
\end{aligned}
$$

which agrees with the general 3-dimensional case provided by Nikravesh [46].

\section{Kane's Method for Dynamic Equation Formulation}

Generating the equations of motion and forces using the Newton-Euler method is unsuitable for this work since it is an inefficient maximal-coordinate method where every active and constraint force for every body must be considered. The Lagrangian method is not more suitable, even if it is a minimal-coordinate method, due to exhaustive partial differentiations in more complex systems such as the one present in this work. Kane's method presents an elegant alternative to these methods. It is a powerful method for generating the dynamics equations for the system through use

of generalized coordinates and partial velocities, and the resulting equations of motion are easily manipulated into a first-order system which lends itself to numerical 
computation and state propagation $[47,48]$. System configuration definition through use of generalized coordinates distinguishes itself from typical Cartesian coordinate definition as the generalized coordinates can be selected to simplify the derivations of equations of motion and reduce the number of equations for the complete system. Kane's method states:

$$
F_{i}+F_{i}^{*}=0, \quad i=1, \ldots, N_{g c}
$$

where Equation 2.11 is the set of $N_{g c}$ scalar equations summing the total inertial forces $F_{i}^{*}$, and active forces $F_{i}$, along the $i$ th generalized coordinate direction, with $N_{g c}$ the number of generalized coordinates required to describe the system. For a system of $N_{B}$ bodies that share the system generalized coordinates $\boldsymbol{q}$, Equation 2.11 can be vectorized and written as the system

$$
0=\boldsymbol{F}+\boldsymbol{F}^{*}
$$

where, summed over the $N_{B}$ bodies in the system, the inertial and active forces are obtained by

$$
\begin{aligned}
\boldsymbol{F} & =\sum_{k=1}^{N_{B}}\left(\left[{ }_{k}^{N} \boldsymbol{V}^{k}\right]^{T} \overrightarrow{\boldsymbol{R}}^{k}+\left[{ }_{k}^{N} \boldsymbol{W}^{k}\right]^{T} \overrightarrow{\boldsymbol{T}}^{k}\right) \\
\boldsymbol{F}^{*} & =\sum_{k=1}^{N_{B}}\left(\left[{ }_{k}^{N} \boldsymbol{V}^{k}\right]^{T} \overrightarrow{\boldsymbol{R}}^{* k}+\left[{ }_{k}^{N} \boldsymbol{W}^{k}\right]^{T} \overrightarrow{\boldsymbol{T}}^{* k}\right)
\end{aligned}
$$

where, for the $k$ th body, $\overrightarrow{\boldsymbol{R}}^{k}$ and $\overrightarrow{\boldsymbol{T}}^{k}$ are the resulting active force and active torque, $\overrightarrow{\boldsymbol{R}}^{* k}$ and $\overrightarrow{\boldsymbol{T}}^{* k}$ are the translational and angular inertias, and $[\boldsymbol{V}]$ and $[\boldsymbol{W}]$ are the translational and angular partial velocity matrices respectively. The partial velocity matrices are obtained from the compact expression of body velocities and accelerations in terms of the generalized velocities vector $\boldsymbol{u}$ and accelerations vector $\dot{\boldsymbol{u}}$ where, for the $k$ th body in the system, we have

$$
\begin{aligned}
{ }_{k}^{N} \overrightarrow{\boldsymbol{v}}^{k} & =\left[{ }_{k}^{N} \boldsymbol{V}^{k}\right] \boldsymbol{u} \\
{ }_{k}^{N} \overrightarrow{\boldsymbol{\omega}}^{k} & =\left[{ }_{k}^{N} \boldsymbol{W}^{k}\right] \boldsymbol{u} \\
{ }_{k}^{N} \overrightarrow{\boldsymbol{a}}^{k} & =\left[{ }_{k}^{N} \boldsymbol{V}^{k}\right] \dot{\boldsymbol{u}}+\overrightarrow{\boldsymbol{Z}}_{k} \\
{ }_{k}^{N} \overrightarrow{\boldsymbol{\alpha}}^{k} & =\left[{ }_{k}^{N} \boldsymbol{W}^{k}\right] \dot{\boldsymbol{u}}+\overrightarrow{\boldsymbol{Y}}_{k}
\end{aligned}
$$

The partial velocity matrices represent the contribution of the system generalized speeds vector $\boldsymbol{u}$, and system generalized accelerations vector $\dot{\boldsymbol{u}}$ to the $k$ th body velocity and acceleration respectively. Coined by Stoneking [49], the terms $\overrightarrow{\boldsymbol{Z}}_{k}$ and $\overrightarrow{\boldsymbol{Y}}_{k}$ 
are the 'remainder translational accelerations' and 'remainder angular accelerations' respectively, and include all kinematic terms which do not include the generalized accelerations.

Likewise, the transpose of the partial velocity matrices are used to project the forces acting on the $N_{B}$ bodies into the permissible motions subspace defined by set of system generalized coordinates $\boldsymbol{q}$ by pre-multiplication of the force vectors as shown in Equations 2.13 and 2.14 [49]. For a single body $k$, Equation 2.12 can be expanded

$$
\begin{aligned}
{\left.\left[{ }_{k}^{N} \boldsymbol{W}^{k}\right]^{T}\left(\overrightarrow{\boldsymbol{T}}^{k}-\left[{ }_{k} \overrightarrow{\boldsymbol{I}}^{k}\right]\left(\left[{ }_{k}^{N} \boldsymbol{W}^{k}\right] \dot{\boldsymbol{u}}+\overrightarrow{\boldsymbol{Y}}_{k}\right)-\left(\widetilde{{ }_{k}^{N} \overrightarrow{\boldsymbol{\omega}}^{k}}\right]\left[{ }_{k} \overrightarrow{\boldsymbol{I}}^{k}\right]{ }_{k}^{N} \overrightarrow{\boldsymbol{\omega}}^{k}\right)\right)+ } \\
{\left[{ }_{k}^{N} \boldsymbol{V}^{k}\right]^{T}\left(\overrightarrow{\boldsymbol{R}}^{k}-m_{k}\left(\left[{ }_{k}^{N} \boldsymbol{V}^{k}\right] \dot{\boldsymbol{u}}+\overrightarrow{\boldsymbol{Z}}_{k}\right)\right)=0 }
\end{aligned}
$$

The vector of unknown generalized accelerations $\dot{\boldsymbol{u}}$ in Equation 2.19 is common to all bodies in the system, and when Kane's equation is summed over the $N_{B}$ bodies in the system, it facilitates the manipulation of the system into the form

$$
\left[\boldsymbol{M}_{\text {sys }}\right] \boldsymbol{x}_{\text {sys }}=\boldsymbol{F}_{\text {sys }}
$$

which lends itself to numerical solvers for obtaining the solution of unknown generalized accelerations for state-propagation purposes.

\section{Dynamic Finite Element Method}

The generic aircraft in the SRAMSS simulation is modelled as a single rigid body helicopter chassis with a skid-type landing gear modelled using a dynamic finite element (DFE) approach. DFE modelling is chosen due to its readily-adaptable modelling of infinitely configurable skid-type landing gear. At a glance, a DFE model mesh physically resembles the skid landing gear which facilitates visual interpretation of the results. More importantly, skid-type landing gear comprises interconnecting, long, slender beams which are easily represented by beam elements in a DFE model.

The DFE model takes the form presented in Bathe [50]:

$$
[\boldsymbol{M}] \ddot{\boldsymbol{q}}+[\boldsymbol{C}] \dot{\boldsymbol{q}}+[\boldsymbol{K}] \boldsymbol{q}=\boldsymbol{F}(\dot{\boldsymbol{q}}, \boldsymbol{q})
$$

where $[\boldsymbol{M}]$ is the consistent mass matrix, $[\boldsymbol{C}]$ is the proportional damping matrix, $[\boldsymbol{K}]$ is the stiffness matrix, $\boldsymbol{F}(\dot{\boldsymbol{q}}, \boldsymbol{q})$ is the vector of externally applied forces on the DFE model, and $\boldsymbol{q}$ and associated time derivatives are the nodal displacements, velocities and accelerations. The mass matrix in the DFE model is constructed as a consistent 
mass matrix, which captures the DFE model node's inertial behavior in all degrees of freedom. The proportional damping matrix is obtained from the first two terms of the Caughey series expansion which satisfies modal superposition [50]:

$$
[\boldsymbol{C}]=[\boldsymbol{M}] \sum_{r=0}^{1} \alpha_{r}\left[[\boldsymbol{M}]^{-1}[\boldsymbol{K}]\right]^{r}
$$

which yields

$$
[\boldsymbol{C}]=\alpha_{0}[\boldsymbol{M}]+\alpha_{1}[\boldsymbol{K}]
$$

where $\alpha_{r}$ are obtained from chosen of frequencies $\omega_{1}, \omega_{2}$ and associated damping ratios $\xi_{1}$ and $\xi_{2}[51]$ :

$$
\xi_{1}=\frac{\alpha_{0}}{2 \omega_{1}}+\frac{\alpha_{1} \omega_{1}}{2} \quad \xi_{2}=\frac{\alpha_{0}}{2 \omega_{2}}+\frac{\alpha_{1} \omega_{2}}{2}
$$

The stiffness matrix is constructed using the direct stiffness method from general 2D 6-DoF beam elements to capture shearing, tension, and bending effects.

\subsection{Dynamic System Definition}

This work develops the planar case of SRAMSS. The system involves the modelling of a generic skid-equipped aircraft with a rigid-body airframe, flexible skid landing gear, and two external dampers joined between the skid cross-tubes and airframe winglets. Four polygons representing the ship deck, RSD deck, and two wedge clamps complete the environment evaluated for contact, and oriented dynamic response, as shown in Figure 2.2.

This represents a system with a maximum of $11+3 N_{n}-r$ DoFs where there are:

- 3 DoF for ship deck motion relative to the inertial frame (heave, sway, roll)

- 1 DoF for RSD deck motion relative to the ship deck (transverse translation)

- 2 DoF of motion per wedge clamp relative to the RSD deck (transverse translation, vertical extension)

- 3 DoF for helicopter motion relative to the inertial frame (heave, sway, roll)

- 3 DoF of motion per DFE node relative to the airframe, minus $r$ fixed-DoFs for the DFE nodes.

The degrees of freedom in the system are described through the use of $7+N_{n}$ frames of references, where $N_{n}$ is the number of DFE nodes and consequently the number of frames required to define the flexible landing gear. The first frame of 


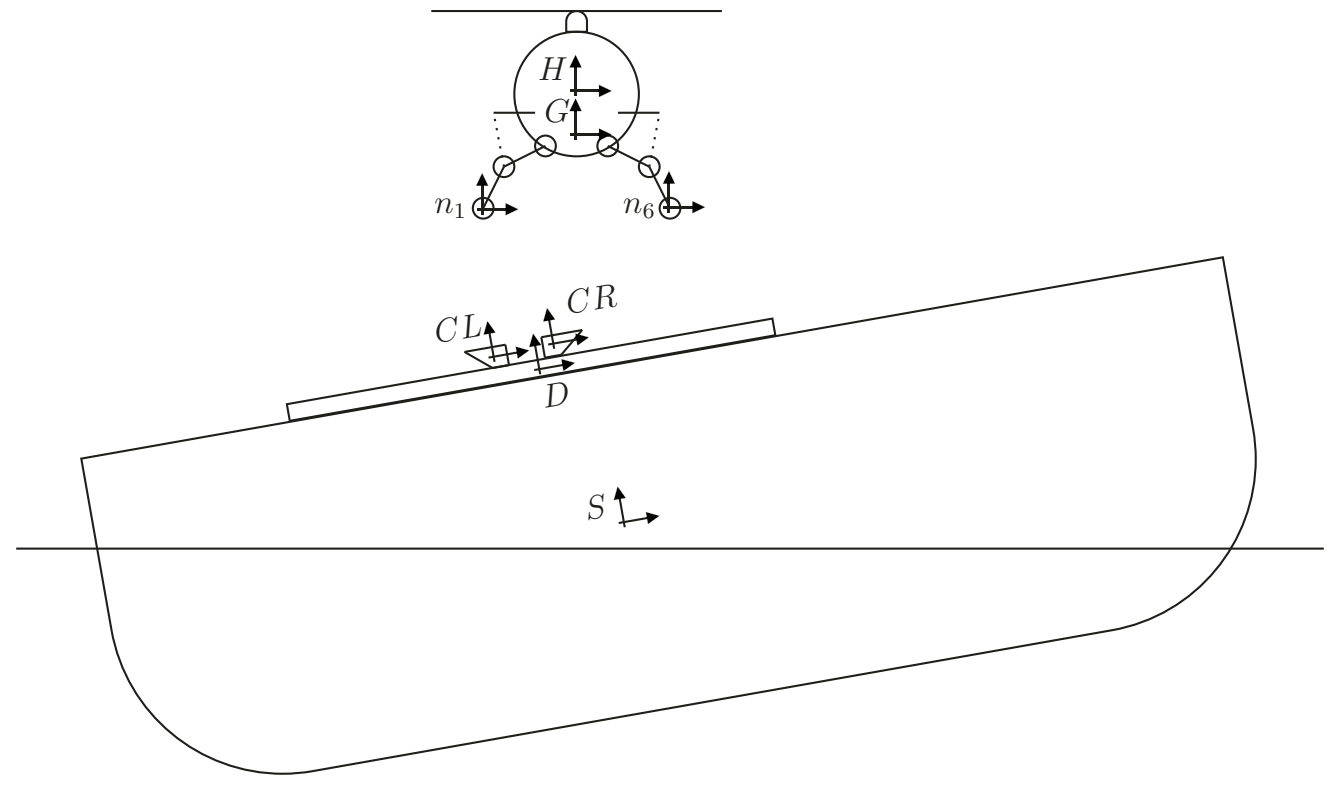

Figure 2.2: Planar description of SRAMSS simulation environment.

reference being the inertial frame $N$ which represents the mean velocity and heading of the ship. Four frames of reference are used as origins for the polygons: $S$ the Ship deck frame of reference, $D$ the RSD deck frame of reference, $C L$ the left wedge clamp frame of reference, and $C R$ the right wedge clamp frame of reference. Three plus $N_{n}$ frames are used to describe the helicopter rigid airframe and flexible elements: $H$ the airframe's frame of reference, $G$ the assembled DFE landing gear's global frame of reference, and $n_{j}$ the $j$ th DFE node's frame of reference. Referring to Figures 2.3 and 2.4 , the frames of reference are setup in chains originating from the inertial frame one chain describes the generic aircraft $\left(N-H-G-n_{j}\right)$, and the other describes the included polygons $(N-S-D-C L-C R)$. Figure 2.4 describes the frames used for the aircraft specifically.

For the complete system there are considerations made with respect to the ordering of the frames of references. There is a fork in the chains of frames to permit the aircraft to move independently from the motion of the ship when generating the equations of motion using Kane's method. The aircraft's frame origin is aligned with the airframe's CoG, with the $z$ axis aligned to the airframe's roll axis, $x$ axis aligned to helicopter's port side, and $y$ axis aligned parallel to the rotor hub axis in the upwards direction. The global DFE frame is axis-aligned to the helicopter frame at a prescribed distance and serves as the interface between the rigid airframe and flexible landing gear. Each DFE node frame is axis-aligned to the global DFE frame, a property required for the 


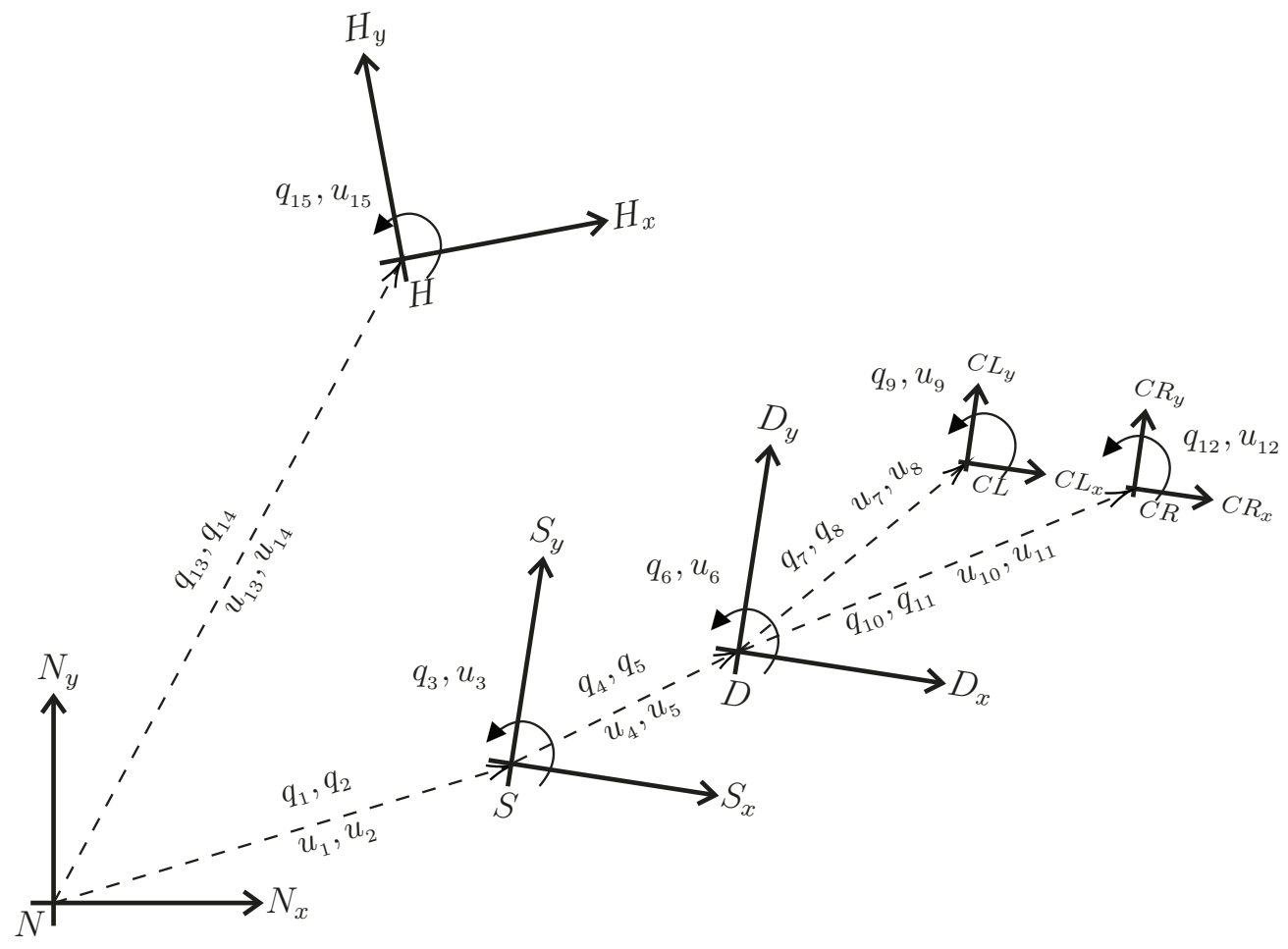

Figure 2.3: System frames of reference.

assembly of the DFE model. Consequently, the transformation matrices between the node frames and global frame are $2 \mathrm{x} 2$ identity matrices. The ship frame is allowed to move in all DoF relative to the inertial frame. The RSD deck frame is axis-aligned at a prescribed distance to the ship frame since its motion is relative to the ship deck. This facilitates manoeuvring prescription by limiting the transverse translation to the $x$ direction. For similar reasons, the wedge clamp frames are axis-aligned to the RSD deck frame to limit prescribed motion to the $x$ and $y$ axes.

The generalized coordinates $\boldsymbol{q}$ and generalized speeds $\boldsymbol{u}$ for the complete system are all consistently defined to be the motion of one frame relative to the previous frame. This facilitates definition of motion and constraints between bodies. Frames which are fixed along certain DoF relative to the previous frame only require those specific generalized coordinates prescribed; and generalized velocities set to zero. Frames with prescribed motions are all defined relative to the frame from which motion would be observed, thus only those generalized coordinates and speeds need to be prescribed.

In this work, the generalized coordinates and speeds for the ship deck, RSD deck, both wedge clamps, and global DFE frame are prescribed. This renders the dynamic system over-determined as some of the generalized accelerations $\dot{\boldsymbol{u}}$ from Equation 2.20 


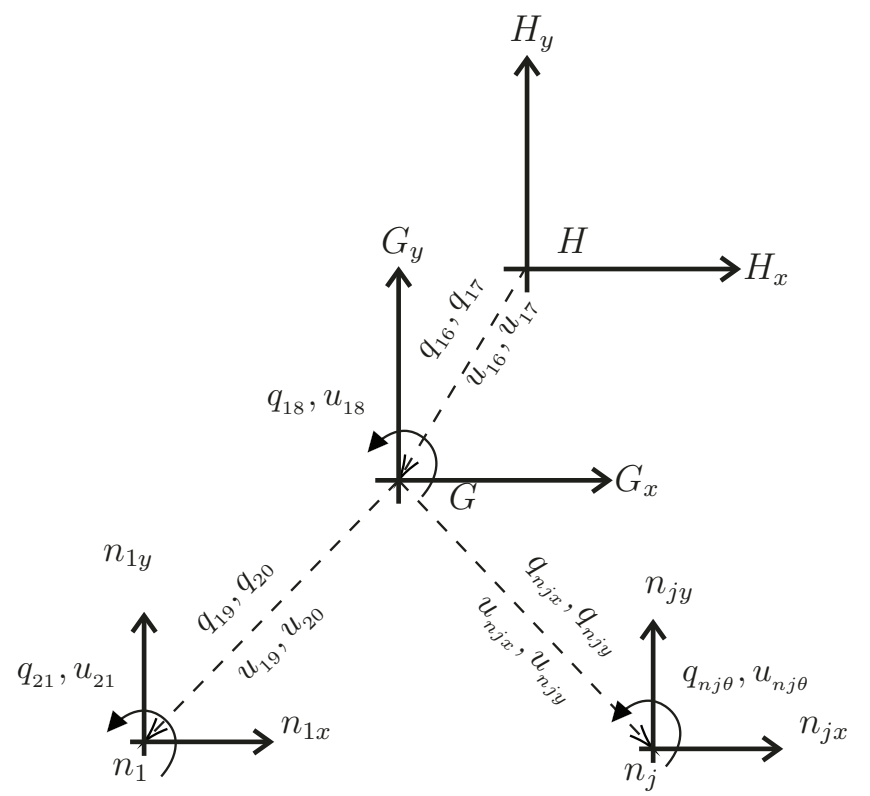

Figure 2.4: Aircraft frames of reference.

become known. Section 5 covers the manipulation of the dynamic system into an exactly-determined system, where a solution for unknown generalized accelerations can be obtained directly.

Regarding the equations of motion for the system, vector quantities must be defined. Starting along the ship's chain of frames, the position of the ship frame relative to the inertial frame, and expressed in the inertial frame, is

$$
{ }_{N}^{N} \overrightarrow{\boldsymbol{r}}^{S}=\left\{\begin{array}{l}
q_{1} \\
q_{2}
\end{array}\right\}
$$

with the orientation of the ship frame relative to the inertial frame, and expressed in the ship frame, being

$$
{ }_{S}^{N} \overrightarrow{\boldsymbol{\theta}}^{S}=\left\{q_{3}\right\}
$$

The position of the RSD deck's frame relative to the ship frame, and expressed in the ship frame, is

$$
{ }_{S}^{S} \overrightarrow{\boldsymbol{r}}^{D}=\left\{\begin{array}{l}
q_{4} \\
q_{5}
\end{array}\right\}
$$

with the orientation of the RSD deck frame relative to the ship frame, and expressed in the RSD deck frame, being

$$
{ }_{D}^{S} \overrightarrow{\boldsymbol{\theta}}^{D}=\left\{q_{6}\right\}
$$


The position of the left wedge frame relative to the RSD deck frame, and expressed in the RSD deck frame, is

$$
{ }_{D}^{D} \overrightarrow{\boldsymbol{r}}^{C L}=\left\{\begin{array}{l}
q_{7} \\
q_{8}
\end{array}\right\}
$$

with the orientation of the left wedge frame relative to the RSD deck frame, and expressed in the left wedge frame, being

$$
{ }_{C L}^{D} \overrightarrow{\boldsymbol{\theta}}^{C L}=\left\{q_{9}\right\}
$$

The position of the right wedge frame relative to the RSD deck frame, and expressed in the RSD deck frame, is

$$
{ }_{D}^{D} \overrightarrow{\boldsymbol{r}}^{C R}=\left\{\begin{array}{l}
q_{10} \\
q_{11}
\end{array}\right\}
$$

with the orientation of the right wedge frame relative to the RSD deck frame, and expressed in the right wedge frame, being

$$
{ }_{C R}^{D} \overrightarrow{\boldsymbol{\theta}}^{C R}=\left\{q_{12}\right\}
$$

Then along the aircraft chain of frames, the position of the helicopter frame relative to the inertial frame, and expressed in the inertial frame, is

$$
{ }_{N}^{N} \overrightarrow{\boldsymbol{r}}^{H}=\left\{\begin{array}{l}
q_{13} \\
q_{14}
\end{array}\right\}
$$

with the orientation of the helicopter frame relative to the inertial frame, and expressed in the helicopter frame, being

$$
{ }_{H}^{N} \overrightarrow{\boldsymbol{\theta}}^{H}=\left\{q_{15}\right\}
$$

The position of the global frame relative to the helicopter frame, and expressed in the helicopter frame, is

$$
{ }_{H}^{H} \overrightarrow{\boldsymbol{r}}^{G}=\left\{\begin{array}{l}
q_{16} \\
q_{17}
\end{array}\right\}
$$

with the orientation of the global frame relative to the helicopter frame, and expressed in the global frame, being

$$
{ }_{G}^{H} \overrightarrow{\boldsymbol{\theta}}^{G}=\left\{q_{18}\right\}
$$

The position of the $j$ th DFE node frame relative to the global frame, and expressed in the global frame, is

$$
{ }_{G}^{G} \overrightarrow{\boldsymbol{r}}^{n_{j}}=\left\{\begin{array}{l}
q_{17+2 j} \\
q_{18+2 j}
\end{array}\right\}
$$


with the orientation of the $j$ th DFE node frame relative to the global frame, and expressed in the $j$ th DFE node frame, being

$$
{ }_{n_{j}}^{G} \overrightarrow{\boldsymbol{\theta}}^{n_{j}}=\left\{q_{19+2 j}\right\}
$$

To generate the correct partial velocity matrices, the generalized velocities are chosen to be the time derivatives of the translational generalized coordinates relative to their preceding frames of reference, and the angular generalized coordinates relative to their respective frames.

The velocity of the ship frame relative to the inertial frame, and expressed in the inertial frame, is

$$
{ }_{N}^{N} \overrightarrow{\boldsymbol{v}}^{S}={ }_{N}^{N} \dot{\overrightarrow{\boldsymbol{r}}}^{S}=\left\{\begin{array}{l}
u_{1} \\
u_{2}
\end{array}\right\}=\left\{\begin{array}{l}
\dot{q}_{1} \\
\dot{q}_{2}
\end{array}\right\}
$$

With the angular velocity of the ship frame relative to the inertial frame, and expressed in the ship frame, being

$$
{ }_{S}^{N} \overrightarrow{\boldsymbol{\omega}}^{S}={ }_{S}^{N} \dot{\overrightarrow{\boldsymbol{\theta}}}^{S}=\left\{u_{3}\right\}=\left\{\dot{q}_{3}\right\}
$$

The velocity of the RSD deck's frame relative to the ship frame, and expressed in the ship frame, is

$$
{ }_{S}^{S} \overrightarrow{\boldsymbol{v}}^{D}={ }_{S}^{S} \dot{\overrightarrow{\boldsymbol{r}}}^{D}=\left\{\begin{array}{l}
u_{4} \\
u_{5}
\end{array}\right\}=\left\{\begin{array}{l}
\dot{q}_{4} \\
\dot{q}_{5}
\end{array}\right\}
$$

with the angular velocity of the RSD deck frame relative to the ship frame, and expressed in the RSD deck frame, being

$$
{ }_{D}^{S} \overrightarrow{\boldsymbol{\omega}}^{D}={ }_{D}^{S} \dot{\overrightarrow{\boldsymbol{\theta}}}^{D}=\left\{u_{6}\right\}=\left\{\dot{q}_{6}\right\}
$$

The velocity of the left wedge frame relative to the RSD deck frame, and expressed in the RSD deck frame, is

$$
{ }_{D}^{D} \overrightarrow{\boldsymbol{v}}^{C L}={ }_{D}^{D} \dot{\overrightarrow{\boldsymbol{r}}} C L=\left\{\begin{array}{l}
u_{7} \\
u_{8}
\end{array}\right\}=\left\{\begin{array}{l}
\dot{q}_{7} \\
\dot{q}_{8}
\end{array}\right\}
$$

with the angular velocity of the left wedge frame relative to the RSD deck frame, and expressed in the left wedge frame, being

$$
{ }_{C L}^{D} \overrightarrow{\boldsymbol{\omega}}^{C L}={ }_{C L}^{D} \dot{\overrightarrow{\boldsymbol{\theta}}}^{C L}=\left\{u_{9}\right\}=\left\{\dot{q}_{9}\right\}
$$


The velocity of the right wedge frame relative to the RSD deck frame, and expressed in the RSD deck frame, is

$$
{ }_{D}^{D} \overrightarrow{\boldsymbol{v}}^{C R}={ }_{D}^{D} \dot{\overrightarrow{\boldsymbol{r}}}^{C R}=\left\{\begin{array}{l}
u_{10} \\
u_{11}
\end{array}\right\}=\left\{\begin{array}{l}
\dot{q}_{10} \\
\dot{q}_{11}
\end{array}\right\}
$$

with the angular velocity of the right wedge frame relative to the RSD deck frame, and expressed in the right wedge frame, being

$$
{ }_{C R}^{D} \overrightarrow{\boldsymbol{\omega}}^{C R}={ }_{C R}^{D} \dot{\overrightarrow{\boldsymbol{\theta}}}^{C R}=\left\{u_{12}\right\}=\left\{\dot{q}_{12}\right\}
$$

Then along the aircraft chain of frames, the velocity of the helicopter frame relative to the inertial frame, and expressed in the inertial frame, is

$$
{ }_{N}^{N} \overrightarrow{\boldsymbol{v}}^{H}={ }_{N}^{N} \dot{\overrightarrow{\boldsymbol{r}}}^{H}=\left\{\begin{array}{l}
u_{13} \\
u_{14}
\end{array}\right\}=\left\{\begin{array}{l}
\dot{q}_{13} \\
\dot{q}_{14}
\end{array}\right\}
$$

with the angular velocity of the helicopter frame relative to the inertial frame, and expressed in the helicopter frame, being

$$
{ }_{H}^{N} \overrightarrow{\boldsymbol{\omega}}^{H}={ }_{H}^{N} \dot{\overrightarrow{\boldsymbol{\theta}}}^{H}=\left\{u_{15}\right\}=\left\{\dot{q}_{15}\right\}
$$

The velocity of the global frame relative to the helicopter frame, and expressed in the helicopter frame, is

$$
{ }_{H}^{H} \overrightarrow{\boldsymbol{v}}^{G}={ }_{H}^{H} \dot{\overrightarrow{\boldsymbol{r}}}^{G}=\left\{\begin{array}{l}
u_{16} \\
u_{17}
\end{array}\right\}=\left\{\begin{array}{l}
\dot{q}_{16} \\
\dot{q}_{17}
\end{array}\right\}
$$

with the angular velocity of the global frame relative to the helicopter frame, and expressed in the global frame, being

$$
{ }_{G}^{H} \overrightarrow{\boldsymbol{\omega}}^{G}={ }_{G}^{H} \dot{\overrightarrow{\boldsymbol{\theta}}}^{G}=\left\{u_{18}\right\}=\left\{\dot{q}_{18}\right\}
$$

The velocity of the $j$ th DFE node frame relative to the global frame, and expressed in the global frame, is

$$
{ }_{G}^{G} \overrightarrow{\boldsymbol{v}}^{n_{j}}={ }_{G}^{G} \dot{\overrightarrow{\boldsymbol{r}}}^{n_{j}}=\left\{\begin{array}{l}
u_{17+2 j} \\
u_{18+2 j}
\end{array}\right\}=\left\{\begin{array}{l}
\dot{q}_{17+2 j} \\
\dot{q}_{18+2 j}
\end{array}\right\}
$$

with the angular velocity of the $j$ th DFE node frame relative to the global frame, and expressed in the $j$ th DFE node frame, being

$$
{ }_{n_{j}}^{G} \overrightarrow{\boldsymbol{\omega}}^{n_{j}}={ }_{n_{j}}^{G} \dot{\overrightarrow{\boldsymbol{\theta}}}^{n_{j}}=\left\{u_{19+2 j}\right\}=\left\{\dot{q}_{19+2 j}\right\}
$$


With all the vector quantities defined through use of generalized coordinates and speeds, the definition of the system is complete. The following Chapter will present the derivation of all equations of motion required both for prescribed and statepropagated bodies, including the partial translational and angular velocity matrices, and remainder accelerations required for the implementation of Kane's method. 


\section{Chapter 3}

\section{System Kinematics}

To prepare for applying Kane's method, as outlined in Section 2.2, translational and angular partial velocity matrices are required. Equations 2.15 through 2.18 show that these matrices are obtained by inspection of the kinematic equations describing a given body.

$$
{ }_{k}^{N} \overrightarrow{\boldsymbol{v}}^{k}=\left[{ }_{k}^{N} \boldsymbol{V}^{k}\right] \boldsymbol{u}
$$

Therefore, the kinematic equations describing the velocity and acceleration of the studied bodies must be derived.

Three important considerations must be made in the derivation of these equations. First, the complete equations of motion must be derived relative to the inertial frame to express absolute accelerations. Second, they must be expressed in the described body's frame of reference since the equations of motion will be evaluated in their respective frames. Lastly, they must be expressed in terms of the generalized speeds and accelerations to isolate the system's vectors of generalized speeds $\boldsymbol{u}$ and accelerations $\dot{\boldsymbol{u}}$ for the construction of respective partial velocity matrices.

For the present work, the equations of motions require kinematic expressions for the airframe and landing gear DFE nodes. Kinematic expressions for ship deck, RSD deck, and wedge clamp polygon vertices are also obtained for contact dynamics purposes. Since the motions of the polygons representing the ship deck, RSD deck, and wedges are prescribed, the partial velocity matrices only require the inclusion of the aircraft's airframe and DFE landing gear node generalized velocities and accelerations. Regardless, the generalized velocities and accelerations for the defined polygons are included in the partial velocity matrices to minimize re-derivations in future work where evaluating the ship's response is required. 
The kinematic development starts with the development of the equations relating to the bodies studied for dynamic response.

\subsection{Body Kinematics}

In this work, the bodies evaluated in Kane's method are the aircraft's airframe and DFE landing gear nodes. Working along the chain of frames of reference for the aircraft, the first body is the aircraft's airframe $H$. Since the $H$ frame is centred on the airframe's CoG, the position of the airframe with respect to the inertial frame, expressed in the inertial frame, is

$$
{ }_{N}^{N} \overrightarrow{\boldsymbol{r}}^{H}
$$

The airframe's velocity is obtained by differentiating Equation 3.1:

$$
\begin{aligned}
{ }_{N}^{N} \overrightarrow{\boldsymbol{v}}^{H} & =\frac{\mathrm{d}}{\mathrm{d} t}\left({ }_{N}^{N} \overrightarrow{\boldsymbol{r}}^{H}\right) \\
& ={ }_{N}^{N} \dot{\overrightarrow{\boldsymbol{r}}}^{H}
\end{aligned}
$$

Transformed to the airframe's frame $H$, the airframe's translational velocity from Equation 3.2 becomes

$$
\begin{aligned}
{ }_{H}^{N} \overrightarrow{\boldsymbol{v}}^{H} & =\left[\boldsymbol{T}_{H \leftarrow N}\right]_{N}^{N} \overrightarrow{\boldsymbol{v}}^{H} \\
& =\left[\boldsymbol{T}_{H \leftarrow N}\right]_{N}^{N} \dot{\overrightarrow{\boldsymbol{r}}}^{H}
\end{aligned}
$$

The airframe's translational acceleration is obtained by differentiating Equation 3.2:

$$
\begin{aligned}
{ }_{N}^{N} \overrightarrow{\boldsymbol{a}}^{H} & =\frac{\mathrm{d}}{\mathrm{d} t}\left(\begin{array}{l}
N \\
N
\end{array} \overrightarrow{\boldsymbol{v}}^{H}\right) \\
& =\frac{\mathrm{d}}{\mathrm{d} t}\left(\begin{array}{l}
N \\
N
\end{array} \dot{\overrightarrow{\boldsymbol{r}}}^{H}\right) \\
& ={ }_{N}^{N} \ddot{\boldsymbol{r}}^{H}
\end{aligned}
$$

Transformed to the airframe's frame $H$, the airframe's translational acceleration from Equation 3.4 becomes

$$
\begin{aligned}
{ }_{H}^{N} \overrightarrow{\boldsymbol{a}}^{H} & =\left[\boldsymbol{T}_{H \leftarrow N}\right]_{N}^{N} \overrightarrow{\boldsymbol{a}}^{H} \\
& =\left[\boldsymbol{T}_{H \leftarrow N}\right]_{N}^{N} \ddot{\overrightarrow{\boldsymbol{r}}}^{H}
\end{aligned}
$$

The next bodies in the aircraft's chain of frames are the DFE nodes representing the landing gear. The frame for each DFE node is located at node coordinates used in the generation of the DFE model. Since all DFE node frames share the common frame 
of reference $G$ per Figure 2.4, the general equation describing the position of the $j$ th DFE node $n_{j}$ with respect to the inertial frame, expressed in the inertial frame is

$$
{ }_{N}^{N} \overrightarrow{\boldsymbol{r}}^{n_{j}}={ }_{N}^{N} \overrightarrow{\boldsymbol{r}}^{H}+{ }_{N}^{H} \overrightarrow{\boldsymbol{r}}^{G}+{ }_{N}^{G} \overrightarrow{\boldsymbol{r}}^{n_{j}}
$$

Equation 3.6 can be described in terms of the generalized coordinates as

$$
{ }_{N}^{N} \overrightarrow{\boldsymbol{r}}^{n_{j}}={ }_{N}^{N} \overrightarrow{\boldsymbol{r}}^{H}+\left[\boldsymbol{T}_{N \leftarrow H}\right]_{H}^{H} \overrightarrow{\boldsymbol{r}}^{G}+\left[\boldsymbol{T}_{N \leftarrow H}\right]\left[\boldsymbol{T}_{H \leftarrow G}\right]_{G}^{G} \overrightarrow{\boldsymbol{r}}^{n_{j}}
$$

The $j$ th DFE node's velocity is obtained by differentiating Equation 3.7:

$$
\begin{aligned}
& { }_{N}^{N} \overrightarrow{\boldsymbol{v}}^{n_{j}}=\frac{\mathrm{d}}{\mathrm{d} t}\left({ }_{N}^{N} \overrightarrow{\boldsymbol{r}}^{n_{j}}\right) \\
& =\frac{\mathrm{d}}{\mathrm{d} t}\left({ }_{N}^{N} \overrightarrow{\boldsymbol{r}}^{H}\right)+\frac{\mathrm{d}}{\mathrm{d} t}\left(\left[\boldsymbol{T}_{N \leftarrow H}\right]_{H}^{H} \overrightarrow{\boldsymbol{r}}^{G}\right)+\frac{\mathrm{d}}{\mathrm{d} t}\left(\left[\boldsymbol{T}_{N \leftarrow H}\right]\left[\boldsymbol{T}_{H \leftarrow G}\right]_{G}^{G} \overrightarrow{\boldsymbol{r}}^{n_{j}}\right) \\
& ={ }_{N}^{N} \dot{\overrightarrow{\boldsymbol{r}}}^{H}+\left[\dot{\boldsymbol{T}}_{N \leftarrow H}\right]_{H}^{H} \overrightarrow{\boldsymbol{r}}^{G}+\left[\boldsymbol{T}_{N \leftarrow H}\right]_{H}^{H} \dot{\overrightarrow{\boldsymbol{r}}}^{G}+\left[\dot{\boldsymbol{T}}_{N \leftarrow H}\right]\left[\boldsymbol{T}_{H \leftarrow G}\right]_{G}^{G} \overrightarrow{\boldsymbol{r}}^{n_{j}} \\
& +\left[\boldsymbol{T}_{N \leftarrow H}\right]\left[\dot{\boldsymbol{T}}_{H \leftarrow G}\right]_{G}^{G} \overrightarrow{\boldsymbol{r}}^{n_{j}}+\left[\boldsymbol{T}_{N \leftarrow H}\right]\left[\boldsymbol{T}_{H \leftarrow G}\right]_{G}^{G} \dot{\overrightarrow{\boldsymbol{r}}}^{n_{j}} \\
& ={ }_{N}^{N} \dot{\overrightarrow{\boldsymbol{r}}}^{H}+\left[\boldsymbol{T}_{N \leftarrow H}\right]\left[\widetilde{{ }_{H}^{N \overrightarrow{\boldsymbol{\omega}}^{H}}}\right]_{H}^{H} \overrightarrow{\boldsymbol{r}}^{G}+\left[\boldsymbol{T}_{N \leftarrow H}\right]_{H}^{H} \dot{\overrightarrow{\boldsymbol{r}}}^{G} \\
& +\left[\boldsymbol{T}_{N \leftarrow H}\right]\left[\widetilde{{ }_{H}^{N \overrightarrow{\boldsymbol{\omega}}^{H}}}\right]\left[\boldsymbol{T}_{H \leftarrow G}\right]_{G}^{G} \overrightarrow{\boldsymbol{r}}^{n_{j}}+\left[\boldsymbol{T}_{N \leftarrow H}\right]\left[\boldsymbol{T}_{H \leftarrow G}\right]\left[\widetilde{H_{G} \overrightarrow{\boldsymbol{\omega}} G}\right]_{G}^{G} \overrightarrow{\boldsymbol{r}}^{n_{j}} \\
& +\left[\boldsymbol{T}_{N \leftarrow H}\right]\left[\boldsymbol{T}_{H \leftarrow G}\right]_{G}^{G} \dot{\overrightarrow{\boldsymbol{r}}}^{n_{j}}
\end{aligned}
$$

By the identity presented in Equation 2.10, the time-derivative of the transformation matrices are substituted by the product of the original transformation matrix and skew angular velocity. Then Equation 3.8 transformed to the $j$ th DFE node's frame $n_{j}$, the $j$ th DFE node's translational velocity from Equation 3.8 becomes

$$
\begin{aligned}
& { }_{n_{j}}^{N} \overrightarrow{\boldsymbol{v}}^{n_{j}}=\left[\boldsymbol{T}_{n_{j} \leftarrow N}\right]_{N}^{N} \overrightarrow{\boldsymbol{v}}^{n_{j}} \\
& =\left[\boldsymbol{T}_{n_{j} \leftarrow N}\right]\left({ }_{N}^{N} \dot{\overrightarrow{\boldsymbol{r}}}^{H}+\left[\boldsymbol{T}_{N \leftarrow H}\right] \widetilde{\left[{ }_{H}^{N \overrightarrow{\boldsymbol{\omega}}^{H}}\right.}\right]_{H}^{H} \boldsymbol{\vec { \boldsymbol { r } }}^{G}+\left[\boldsymbol{T}_{N \leftarrow H}\right]_{H}^{H} \dot{\overrightarrow{\boldsymbol{r}}}^{G} \\
& +\left[\boldsymbol{T}_{N \leftarrow H}\right]\left[\widetilde{\left.{ }_{H}^{N \overrightarrow{\boldsymbol{\omega}}^{H}}\right]}\left[\boldsymbol{T}_{H \leftarrow G}\right]_{G}^{G} \overrightarrow{\boldsymbol{r}}^{n_{j}}+\left[\boldsymbol{T}_{N \leftarrow H}\right]\left[\boldsymbol{T}_{H \leftarrow G}\right] \widetilde{{ }_{G}^{H} \overrightarrow{\boldsymbol{\omega}} G}\right]_{G}^{G} \overrightarrow{\boldsymbol{r}}^{n_{j}} \\
& \left.+\left[\boldsymbol{T}_{N \leftarrow H}\right]\left[\boldsymbol{T}_{H \leftarrow G}\right]_{G}^{G} \dot{\overrightarrow{\boldsymbol{r}}}^{n_{j}}\right) \\
& =\left[\boldsymbol{T}_{n_{j} \leftarrow N}\right]_{N}^{N} \dot{\overrightarrow{\boldsymbol{r}}}^{H}+\left[\boldsymbol{T}_{n_{j} \leftarrow H}\right]\left[\widetilde{N_{H} \overrightarrow{\boldsymbol{\omega}}^{H}}\right]_{H}^{H} \overrightarrow{\boldsymbol{r}}^{G}+\left[\boldsymbol{T}_{n_{j} \leftarrow H}\right]_{H}^{H} \dot{\overrightarrow{\boldsymbol{r}}}^{G} \\
& +\left[\boldsymbol{T}_{n_{j} \leftarrow H}\right] \widetilde{\left[{ }_{H}^{N \overrightarrow{\boldsymbol{\omega}}^{H}}\right]}\left[\boldsymbol{T}_{H \leftarrow G}\right]_{G}^{G} \overrightarrow{\boldsymbol{r}}^{n_{j}}+\left[\boldsymbol{T}_{n_{j} \leftarrow G}\right]\left[\widetilde{{ }_{G}^{H} \overrightarrow{\boldsymbol{\omega}} G}\right]_{G}^{G} \overrightarrow{\boldsymbol{r}}^{n_{j}} \\
& +\left[\boldsymbol{T}_{n_{j} \leftarrow G}\right]_{G}^{G} \dot{\overrightarrow{\boldsymbol{r}}} n_{j} \\
& =\left[\boldsymbol{T}_{n_{j} \leftarrow N}\right]_{N}^{N} \dot{\overrightarrow{\boldsymbol{r}}}^{H}-\left[\boldsymbol{T}_{n_{j} \leftarrow H}\right]\left[\widetilde{{ }_{H}^{H} \overrightarrow{\boldsymbol{r}} G}\right]_{H}^{N} \overrightarrow{\boldsymbol{\omega}}^{H}+\left[\boldsymbol{T}_{n_{j} \leftarrow H}\right]_{H}^{H} \dot{\overrightarrow{\boldsymbol{r}}}^{G}
\end{aligned}
$$

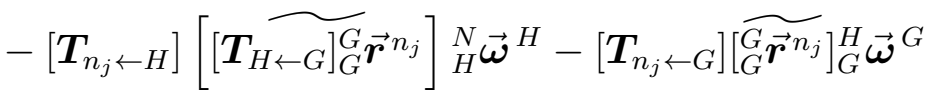

$$
\begin{aligned}
& +\left[\boldsymbol{T}_{n_{j} \leftarrow G}\right]_{G}^{G} \dot{\overrightarrow{\boldsymbol{r}}} n_{j}
\end{aligned}
$$


where the angular velocities ${ }_{H}^{N} \overrightarrow{\boldsymbol{\omega}}^{H}$ and ${ }_{G}^{H} \overrightarrow{\boldsymbol{\omega}}^{G}$, composed of generalized speeds, are expressed in vector form to enable the formation of the partial velocity matrices.

The $j$ th DFE node's translational acceleration is obtained by differentiating Equation 3.8:

$$
\begin{aligned}
& { }_{N}^{N} \overrightarrow{\boldsymbol{a}}^{n_{j}}=\frac{\mathrm{d}}{\mathrm{d} t}\left({ }_{N}^{N} \overrightarrow{\boldsymbol{v}}^{n_{j}}\right) \\
& =\frac{\mathrm{d}}{\mathrm{d} t}\left({ }_{N}^{N} \dot{\overrightarrow{\boldsymbol{r}}}^{H}\right)+\frac{\mathrm{d}}{\mathrm{d} t}\left(\left[\boldsymbol{T}_{N \leftarrow H}\right]\left[\widetilde{{ }_{H} \overrightarrow{\boldsymbol{\omega}}^{H}}\right]_{H}^{H} \overrightarrow{\boldsymbol{r}}^{G}\right) \\
& +\frac{\mathrm{d}}{\mathrm{d} t}\left(\left[\boldsymbol{T}_{N \leftarrow H}\right]_{H}^{H} \dot{\overrightarrow{\boldsymbol{r}}}^{G}\right)+\frac{\mathrm{d}}{\mathrm{d} t}\left(\left[\boldsymbol{T}_{N \leftarrow H}\right]\left[\widetilde{{ }_{H}^{N \overrightarrow{\boldsymbol{\omega}}^{H}}}\right]\left[\boldsymbol{T}_{H \leftarrow G}\right]_{G}^{G} \overrightarrow{\boldsymbol{r}}^{n_{j}}\right) \\
& +\frac{\mathrm{d}}{\mathrm{d} t}\left(\left[\boldsymbol{T}_{N \leftarrow H}\right]\left[\boldsymbol{T}_{H \leftarrow G}\right]\left[\widetilde{\left[{ }_{G}^{H} \overrightarrow{\boldsymbol{\omega}}^{G}\right.}\right]_{G}^{G} \overrightarrow{\boldsymbol{r}}^{n_{j}}\right) \\
& +\frac{\mathrm{d}}{\mathrm{d} t}\left(\left[\boldsymbol{T}_{N \leftarrow H}\right]\left[\boldsymbol{T}_{H \leftarrow G}\right]_{G}^{G} \dot{\overrightarrow{\boldsymbol{r}}}^{n_{j}}\right) \\
& ={ }_{N}^{N} \ddot{\overrightarrow{\boldsymbol{r}}}{ }^{H}+\left[\dot{\boldsymbol{T}}_{N \leftarrow H}\right]\left[\widetilde{N_{H}^{N} \overrightarrow{\boldsymbol{\omega}}^{H}}\right]_{H}^{H} \overrightarrow{\boldsymbol{r}}^{G}+\left[\boldsymbol{T}_{N \leftarrow H}\right] \widetilde{\left[{ }_{H}^{N \dot{\overrightarrow{\boldsymbol{\omega}}}}\right]_{H}^{H}} \overrightarrow{\boldsymbol{r}}^{G} \\
& +\left[\boldsymbol{T}_{N \leftarrow H}\right]\left[\widetilde{N_{H}^{N \overrightarrow{\boldsymbol{\omega}}} H}\right]_{H}^{H} \dot{\overrightarrow{\boldsymbol{r}}}^{G}+\left[\dot{\boldsymbol{T}}_{N \leftarrow H}\right]_{H}^{H} \dot{\overrightarrow{\boldsymbol{r}}}^{G}+\left[\boldsymbol{T}_{N \leftarrow H}\right]_{H}^{H} \ddot{\overrightarrow{\boldsymbol{r}}} G \\
& +\left[\dot{\boldsymbol{T}}_{N \leftarrow H}\right] \widetilde{\left.{ }_{H}^{N \overrightarrow{\boldsymbol{\omega}}^{H}}\right]}\left[\boldsymbol{T}_{H \leftarrow G}\right]_{G}^{G} \overrightarrow{\boldsymbol{r}}^{n_{j}}+\left[\boldsymbol{T}_{N \leftarrow H}\right]\left[\widetilde{\left.{ }_{H}^{N \dot{\overrightarrow{\boldsymbol{\omega}}}^{H}}\right]}\left[\boldsymbol{T}_{H \leftarrow G}\right]_{G}^{G} \overrightarrow{\boldsymbol{r}}^{n_{j}}\right. \\
& +\left[\boldsymbol{T}_{N \leftarrow H}\right]\left[\widetilde{{ }_{H}^{N \overrightarrow{\boldsymbol{\omega}}^{H}}}\right]\left[\dot{\boldsymbol{T}}_{H \leftarrow G}\right]_{G}^{G} \overrightarrow{\boldsymbol{r}}^{n_{j}}+\left[\boldsymbol{T}_{N \leftarrow H}\right]\left[\widetilde{{ }_{H}^{N \overrightarrow{\boldsymbol{\omega}}^{H}}}\right]\left[\boldsymbol{T}_{H \leftarrow G}\right]_{G}^{G} \dot{\overrightarrow{\boldsymbol{r}}}^{n_{j}} \\
& +\left[\dot{\boldsymbol{T}}_{N \leftarrow H}\right]\left[\boldsymbol{T}_{H \leftarrow G}\right]\left[\widetilde{{ }_{G}^{H} \overrightarrow{\boldsymbol{\omega}}^{G}}\right]_{G}^{G} \overrightarrow{\boldsymbol{r}}^{n_{j}}+\left[\boldsymbol{T}_{N \leftarrow H}\right]\left[\dot{\boldsymbol{T}}_{H \leftarrow G}\right]\left[\widetilde{{ }_{G}^{H} \overrightarrow{\boldsymbol{\omega}} G}\right]_{G}^{G} \overrightarrow{\boldsymbol{r}}^{n_{j}} \\
& \left.+\left[\boldsymbol{T}_{N \leftarrow H}\right]\left[\boldsymbol{T}_{H \leftarrow G}\right] \widetilde{\left[{ }_{G}^{H} \tilde{\dot{\boldsymbol{\omega}}} G\right.}\right]_{G}^{G} \overrightarrow{\boldsymbol{r}}^{n_{j}}+\left[\boldsymbol{T}_{N \leftarrow H}\right]\left[\boldsymbol{T}_{H \leftarrow G}\right]\left[\widetilde{{ }_{G}^{H} \overrightarrow{\boldsymbol{\omega}} G}\right]_{G}^{G} \dot{\overrightarrow{\boldsymbol{r}}} n_{j} \\
& +\left[\dot{\boldsymbol{T}}_{N \leftarrow H}\right]\left[\boldsymbol{T}_{H \leftarrow G}\right]_{G}^{G} \dot{\overrightarrow{\boldsymbol{r}}}^{n_{j}}+\left[\boldsymbol{T}_{N \leftarrow H}\right]\left[\dot{\boldsymbol{T}}_{H \leftarrow G}\right]_{G}^{G} \dot{\overrightarrow{\boldsymbol{r}}}^{n_{j}} \\
& +\left[\boldsymbol{T}_{N \leftarrow H}\right]\left[\boldsymbol{T}_{H \leftarrow G}\right]_{G}^{G} \ddot{\overrightarrow{\boldsymbol{r}}} n_{j}
\end{aligned}
$$

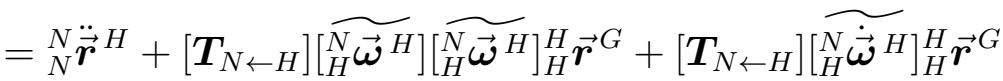

$$
\begin{aligned}
& +2\left[\boldsymbol{T}_{N \leftarrow H}\right]\left[\widetilde{{ }_{H}^{N \overrightarrow{\boldsymbol{\omega}}^{H}}}\right]_{H}^{H} \dot{\overrightarrow{\boldsymbol{r}}}^{G}+\left[\boldsymbol{T}_{N \leftarrow H}\right]_{H}^{H} \ddot{\overrightarrow{\boldsymbol{r}}}^{G} \\
& +\left[\boldsymbol{T}_{N \leftarrow H}\right]\left[\widetilde{N_{H}^{N \overrightarrow{\boldsymbol{\omega}}^{H}}}\right]\left[\widetilde{N_{H}^{N \overrightarrow{\boldsymbol{\omega}}^{H}}}\right]\left[\boldsymbol{T}_{H \leftarrow G}\right]_{G}^{G} \overrightarrow{\boldsymbol{r}}^{n_{j}}+\left[\boldsymbol{T}_{N \leftarrow H}\right]\left[\widetilde{N_{H}^{N}} \widetilde{\overrightarrow{\boldsymbol{\omega}}}^{H}\right]\left[\boldsymbol{T}_{H \leftarrow G}\right]_{G}^{G} \overrightarrow{\boldsymbol{r}}^{n_{j}}
\end{aligned}
$$

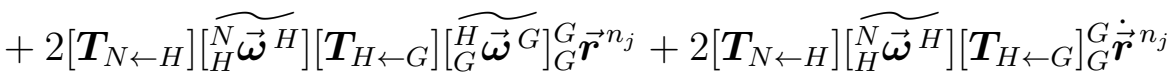

$$
\begin{aligned}
& \left.+\left[\boldsymbol{T}_{N \leftarrow H}\right]\left[\boldsymbol{T}_{H \leftarrow G}\right] \widetilde{\left[{ }_{G}^{H} \overrightarrow{\boldsymbol{\omega}}^{G}\right.}\right]\left[\widetilde{{ }_{G}^{H} \overrightarrow{\boldsymbol{\omega}}^{G}}\right]_{G}^{G} \overrightarrow{\boldsymbol{r}}^{n_{j}}+\left[\boldsymbol{T}_{N \leftarrow H}\right]\left[\boldsymbol{T}_{H \leftarrow G}\right]\left[\widetilde{{ }_{G}^{H \dot{\boldsymbol{\omega}}} G}\right]_{G}^{G} \overrightarrow{\boldsymbol{r}}^{n_{j}} \\
& +2\left[\boldsymbol{T}_{N \leftarrow H}\right]\left[\boldsymbol{T}_{H \leftarrow G}\right]\left[\widetilde{{ }_{G}^{H} \overrightarrow{\boldsymbol{\omega}} G}\right]_{G}^{G} \dot{\overrightarrow{\boldsymbol{r}}}^{n_{j}}+\left[\boldsymbol{T}_{N \leftarrow H}\right]\left[\boldsymbol{T}_{H \leftarrow G}\right]_{G}^{G} \ddot{\overrightarrow{\boldsymbol{r}}} n_{j}
\end{aligned}
$$

Transformed to the $j$ th DFE node's frame $n_{j}$, the $j$ th DFE node's translational 
acceleration from Equation 3.10 becomes

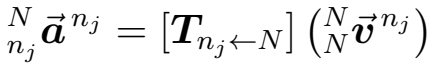

$$
\begin{aligned}
& =\left[\boldsymbol{T}_{n_{j} \leftarrow N}\right]\left({ }_{N}^{N} \ddot{\overrightarrow{\boldsymbol{r}}}{ }^{H}+\left[\boldsymbol{T}_{N \leftarrow H}\right]\left[\widetilde{{ }_{H}^{N} \overrightarrow{\boldsymbol{\omega}}^{H}}\right]\left[\widetilde{{ }_{H}^{N} \overrightarrow{\boldsymbol{\omega}}^{H}}\right]_{H}^{H} \overrightarrow{\boldsymbol{r}}^{G}+\left[\boldsymbol{T}_{N \leftarrow H}\right] \widetilde{\left[{ }_{H}^{N} \dot{\overrightarrow{\boldsymbol{\omega}}}^{H}\right]_{H}^{H}} \overrightarrow{\boldsymbol{r}}^{G}\right. \\
& \left.+2\left[\boldsymbol{T}_{N \leftarrow H}\right] \widetilde{\left[{ }_{H}^{N \overrightarrow{\boldsymbol{\omega}}} H\right.}\right]_{H}^{H} \dot{\overrightarrow{\boldsymbol{r}}} G+\left[\boldsymbol{T}_{N \leftarrow H}\right]_{H}^{H} \ddot{\overrightarrow{\boldsymbol{r}}} G \\
& \left.\left.+\left[\boldsymbol{T}_{N \leftarrow H}\right]\left[\widetilde{N_{H}^{N} \overrightarrow{\boldsymbol{\omega}}^{H}}\right] \widetilde{N_{H}^{N \overrightarrow{\boldsymbol{\omega}}^{H}}}\right]\left[\boldsymbol{T}_{H \leftarrow G}\right]_{G}^{G} \overrightarrow{\boldsymbol{r}}^{n_{j}}+\left[\boldsymbol{T}_{N \leftarrow H}\right] \widetilde{\stackrel{N}{H}_{\dot{\overrightarrow{\boldsymbol{\omega}}} H}^{H}}\right]\left[\boldsymbol{T}_{H \leftarrow G}\right]_{G}^{G} \overrightarrow{\boldsymbol{r}}^{n_{j}} \\
& +2\left[\boldsymbol{T}_{N \leftarrow H}\right]\left[\widetilde{N_{H}^{(\overrightarrow{\boldsymbol{\omega}} H}}\right]\left[\boldsymbol{T}_{H \leftarrow G}\right]\left[\widetilde{H_{G}^{H} \overrightarrow{\boldsymbol{\omega}}^{G}}\right]_{G}^{G} \overrightarrow{\boldsymbol{r}}^{n_{j}}+2\left[\boldsymbol{T}_{N \leftarrow H}\right]\left[\widetilde{N_{H}^{N} \overrightarrow{\boldsymbol{\omega}}^{H}}\right]\left[\boldsymbol{T}_{H \leftarrow G}\right]_{G}^{G} \dot{\overrightarrow{\boldsymbol{r}}}^{n_{j}} \\
& \left.\left.+\left[\boldsymbol{T}_{N \leftarrow H}\right]\left[\boldsymbol{T}_{H \leftarrow G}\right]\left[\widetilde{{ }_{G}^{H} \overrightarrow{\boldsymbol{\omega}}^{G}}\right] \widetilde{{ }_{G}^{H} \overrightarrow{\boldsymbol{\omega}} G}\right]_{G}^{G} \overrightarrow{\boldsymbol{r}}^{n_{j}}+\left[\boldsymbol{T}_{N \leftarrow H}\right]\left[\boldsymbol{T}_{H \leftarrow G}\right] \widetilde{\left[{ }_{G}^{H \dot{\boldsymbol{\omega}}} G\right.}\right]_{G}^{G} \overrightarrow{\boldsymbol{r}}^{n_{j}} \\
& \left.+2\left[\boldsymbol{T}_{N \leftarrow H}\right]\left[\boldsymbol{T}_{H \leftarrow G}\right]\left[\widetilde{H_{G} \overrightarrow{\boldsymbol{\omega}} G}\right]_{G}^{G} \dot{\overrightarrow{\boldsymbol{r}}} n_{j}+\left[\boldsymbol{T}_{N \leftarrow H}\right]\left[\boldsymbol{T}_{H \leftarrow G}\right]_{G}^{G} \ddot{\overrightarrow{\boldsymbol{r}}} n_{j}\right)
\end{aligned}
$$

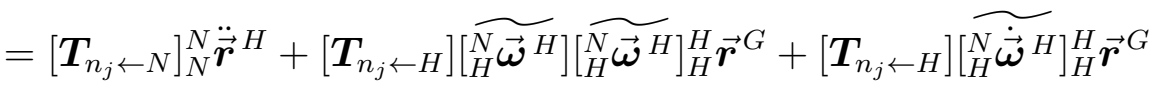

$$
\begin{aligned}
& +2\left[\boldsymbol{T}_{n_{j} \leftarrow H}\right]\left[\widetilde{{ }_{H}^{N} \overrightarrow{\boldsymbol{\omega}} H}\right]_{H}^{H} \dot{\overrightarrow{\boldsymbol{r}}}^{G}+\left[\boldsymbol{T}_{n_{j} \leftarrow H}\right]_{H}^{H} \ddot{\overrightarrow{\boldsymbol{r}}} G
\end{aligned}
$$

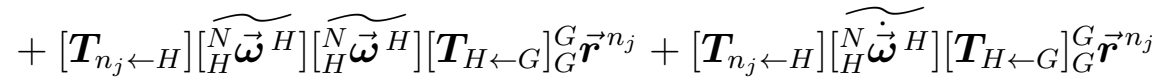

$$
\begin{aligned}
& \left.\left.+2\left[\boldsymbol{T}_{n_{j} \leftarrow H}\right]\left[\widetilde{{ }_{H}^{N} \overrightarrow{\boldsymbol{\omega}}^{H}}\right]\left[\boldsymbol{T}_{H \leftarrow G}\right] \widetilde{{ }_{G}^{H} \overrightarrow{\boldsymbol{\omega}}^{G}}\right]_{G}^{G} \overrightarrow{\boldsymbol{r}}^{n_{j}}+2\left[\boldsymbol{T}_{n_{j} \leftarrow H}\right] \widetilde{{ }_{H}^{N \overrightarrow{\boldsymbol{\omega}}^{H}}}\right]\left[\boldsymbol{T}_{H \leftarrow G}\right]_{G}^{G} \dot{\overrightarrow{\boldsymbol{r}}} n_{j} \\
& \left.+\left[\boldsymbol{T}_{n_{j} \leftarrow G}\right]\left[\widetilde{\left[{ }_{G}^{H} \overrightarrow{\boldsymbol{\omega}}^{G}\right.}\right] \widetilde{{ }_{G}^{H} \overrightarrow{\boldsymbol{\omega}}^{G}}\right]_{G}^{G} \overrightarrow{\boldsymbol{r}}^{n_{j}}+\left[\boldsymbol{T}_{n_{j} \leftarrow G}\right] \widetilde{\left[{ }_{G}^{H} \dot{\overrightarrow{\boldsymbol{\omega}}}^{G}\right]_{G}^{G}} \overrightarrow{\boldsymbol{r}}^{n_{j}} \\
& \left.+2\left[\boldsymbol{T}_{n_{j} \leftarrow G}\right] \widetilde{{ }_{G}^{H} \overrightarrow{\boldsymbol{\omega}} G}\right]_{G}^{G} \dot{\overrightarrow{\boldsymbol{r}}} n_{j}+\left[\boldsymbol{T}_{n_{j} \leftarrow G}\right]_{G}^{G} \ddot{\overrightarrow{\boldsymbol{r}}} n_{j}
\end{aligned}
$$

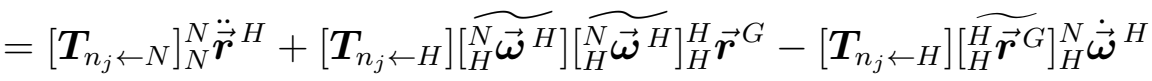

$$
\begin{aligned}
& +2\left[\boldsymbol{T}_{n_{j} \leftarrow H}\right]\left[\widetilde{N_{H}^{N} \overrightarrow{\boldsymbol{\omega}}^{H}}\right]_{H}^{H} \dot{\overrightarrow{\boldsymbol{r}}} G+\left[\boldsymbol{T}_{n_{j} \leftarrow H}\right]_{H}^{H} \ddot{\overrightarrow{\boldsymbol{r}}} G \\
& +\left[\boldsymbol{T}_{n_{j} \leftarrow H}\right] \widetilde{\left.{ }_{H}^{N \overrightarrow{\boldsymbol{\omega}}^{H}}\right]}\left[\widetilde{{ }_{H}^{N \overrightarrow{\boldsymbol{\omega}}^{H}}}\right]\left[\boldsymbol{T}_{H \leftarrow G}\right]_{G}^{G} \overrightarrow{\boldsymbol{r}}^{n_{j}}-\left[\boldsymbol{T}_{n_{j} \leftarrow H}\right]\left[\left[\widetilde{\left.\boldsymbol{T}_{H \leftarrow G}\right]_{G}^{G}} \overrightarrow{\boldsymbol{r}}^{n_{j}}\right] \underset{H}{N} \dot{\overrightarrow{\boldsymbol{\omega}}}^{H}\right. \\
& +2\left[\boldsymbol{T}_{n_{j} \leftarrow H}\right]\left[\widetilde{{ }_{H}^{N \overrightarrow{\boldsymbol{\omega}}^{H}}}\right]\left[\boldsymbol{T}_{H \leftarrow G}\right]\left[\widetilde{\left[{ }_{G}^{H} \overrightarrow{\boldsymbol{\omega}}^{G}\right.}\right]_{G}^{G} \overrightarrow{\boldsymbol{r}}^{n_{j}}+2\left[\boldsymbol{T}_{n_{j} \leftarrow H}\right]\left[\widetilde{{ }_{H}^{N \overrightarrow{\boldsymbol{\omega}}^{H}}}\right]\left[\boldsymbol{T}_{H \leftarrow G}\right]_{G}^{G} \dot{\overrightarrow{\boldsymbol{r}}}^{n_{j}} \\
& +\left[\widetilde{\left.\boldsymbol{T}_{n_{j} \leftarrow G}\right]\left[\widetilde{{ }_{G}^{H} \overrightarrow{\boldsymbol{\omega}} G}\right]\left[\widetilde{H_{G}^{H} \overrightarrow{\boldsymbol{\omega}}^{G}}\right]_{G}^{G} \overrightarrow{\boldsymbol{r}}^{n_{j}}-\left[\boldsymbol{T}_{n_{j} \leftarrow G}\right]\left[\widetilde{{ }_{G}^{G} \overrightarrow{\boldsymbol{r}}^{n_{j}}}\right]_{G}^{H}} \dot{\overrightarrow{\boldsymbol{\omega}}}^{G}\right. \\
& +2\left[\boldsymbol{T}_{n_{j} \leftarrow G}\right]\left[\widetilde{{ }_{G}^{H} \overrightarrow{\boldsymbol{\omega}} G}\right]_{G}^{G} \dot{\overrightarrow{\boldsymbol{r}}} n_{j}+\left[\boldsymbol{T}_{n_{j} \leftarrow G}\right]_{G}^{G} \ddot{\overrightarrow{\boldsymbol{r}}} n_{j}
\end{aligned}
$$

where the angular accelerations ${ }_{H}^{N} \dot{\overrightarrow{\boldsymbol{\omega}}}^{H}$ and ${ }_{G}^{H} \dot{\overrightarrow{\boldsymbol{\omega}}}^{G}$, composed of generalized accelerations, are expressed in vector form to enable the formation of the partial velocity matrices.

Equations 3.1 through 3.11 represent the complete translational kinematic expressions for the bodies studied in this system. Again, working along the chain of frames of reference for the aircraft, the first body is the aircraft's airframe $H$. The orientation of the body with respect to the inertial frame, expressed in the inertial frame, 
is

$$
\begin{aligned}
{ }_{N}^{N} \overrightarrow{\boldsymbol{\theta}}^{H} & =\left[\boldsymbol{T}_{N \leftarrow H}\right]_{H}^{N} \overrightarrow{\boldsymbol{\theta}}^{H} \\
& =[\boldsymbol{I}]_{1 \times 1}{ }_{H}^{N} \overrightarrow{\boldsymbol{\theta}}^{H} \\
& ={ }_{H}^{N} \overrightarrow{\boldsymbol{\theta}}^{H}
\end{aligned}
$$

Note the planar case's property where the relative angle to the previous frame is the same regardless of the frame in which it is expressed due to the transformation matrix being identity as shown in Equation 2.7. For the fully-spatial case of SRAMSS, such simplifications cannot be made.

The airframe's angular velocity is obtained by differentiating Equation 3.1:

$$
\begin{aligned}
{ }_{N}^{N} \overrightarrow{\boldsymbol{\omega}}^{H} & =\frac{\mathrm{d}}{\mathrm{d} t}\left({ }_{N}^{N} \overrightarrow{\boldsymbol{\theta}}^{H}\right) \\
& =\frac{\mathrm{d}}{\mathrm{d} t}\left(\left[\boldsymbol{T}_{N \leftarrow H}\right]_{H}^{N} \overrightarrow{\boldsymbol{\theta}}^{H}\right) \\
& =\left[\boldsymbol{T}_{N \leftarrow H}\right]_{H}^{N} \dot{\overrightarrow{\boldsymbol{\theta}}}^{H} \\
& =\left[\boldsymbol{T}_{N \leftarrow H}\right]_{H}^{N} \overrightarrow{\boldsymbol{\omega}}^{H} \\
& =[\boldsymbol{I}]_{1 \times 1}{ }_{H}^{N} \overrightarrow{\boldsymbol{\omega}}^{H} \\
& ={ }_{H}^{N} \overrightarrow{\boldsymbol{\omega}}^{H}
\end{aligned}
$$

Similarly to Equation 3.12, because ${ }_{N}^{N} \overrightarrow{\boldsymbol{\theta}}^{H} \equiv{ }_{H}^{N} \overrightarrow{\boldsymbol{\theta}}^{H}$, Equation 3.13 finds that ${ }_{N}^{N} \overrightarrow{\boldsymbol{\omega}}^{H} \equiv$ ${ }_{H}^{N} \overrightarrow{\boldsymbol{\omega}}^{H}$ for the planar case. Considering that the partial velocity matrices are expressed in the body frames, ${ }_{H}^{N} \overrightarrow{\boldsymbol{\omega}}^{H}$ is the preferred notation.

The airframe's angular acceleration is obtained by differentiating Equation 3.13:

$$
\begin{aligned}
{ }_{N}^{N} \overrightarrow{\boldsymbol{\alpha}}^{H} & =\frac{\mathrm{d}}{\mathrm{d} t}\left({ }_{N}^{N} \overrightarrow{\boldsymbol{\omega}}^{H}\right) \\
& =\frac{\mathrm{d}}{\mathrm{d} t}\left(\left[\boldsymbol{T}_{N \leftarrow H}\right]_{H}^{N} \overrightarrow{\boldsymbol{\omega}}^{H}\right) \\
& =\left[\dot{\boldsymbol{T}}_{N \leftarrow H}\right]_{H}^{N} \overrightarrow{\boldsymbol{\omega}}^{H}+\left[\boldsymbol{T}_{N \leftarrow H}\right]_{H}^{N} \dot{\overrightarrow{\boldsymbol{\omega}}}^{H} \\
& \left.=\left[\boldsymbol{T}_{N \leftarrow H}\right]_{H}^{N \overrightarrow{\boldsymbol{\omega}}^{H}}\right]_{H}^{N} \overrightarrow{\boldsymbol{\omega}}^{H}+\left[\boldsymbol{T}_{N \leftarrow H}\right]_{H}^{N} \dot{\overrightarrow{\boldsymbol{\omega}}}^{H} \\
& =[\boldsymbol{I}]_{1 \times 1}^{N} \overrightarrow{\boldsymbol{\alpha}}^{H} \\
& ={ }_{H}^{N} \overrightarrow{\boldsymbol{\alpha}}^{H}
\end{aligned}
$$

Once more, equivalence between frames of references is observed; ${ }_{N}^{N} \overrightarrow{\boldsymbol{\alpha}}^{H} \equiv{ }_{H}^{N} \overrightarrow{\boldsymbol{\alpha}}^{H}$ for the planar case. Considering that the partial velocity matrices are expressed in the body frames, ${ }_{H}^{N} \overrightarrow{\boldsymbol{\alpha}}^{H}$ is the preferred notation. 
The next body in the aircraft's chain of frame's is the $j$ th DFE node $n_{j}$. The general equation describing the orientation of the $j$ th DFE node frame with respect to the inertial frame, expressed in the inertial frame is

$$
\begin{aligned}
{ }_{N}^{N} \overrightarrow{\boldsymbol{\theta}}^{n_{j}} & ={ }_{N}^{N} \overrightarrow{\boldsymbol{\theta}}^{H}+{ }_{N}^{H} \overrightarrow{\boldsymbol{\theta}}^{G}+{ }_{N}^{G} \overrightarrow{\boldsymbol{\theta}}^{n_{j}} \\
& =\left[\boldsymbol{T}_{N \leftarrow H}\right]_{H}^{N} \overrightarrow{\boldsymbol{\theta}}^{H}+\left[\boldsymbol{T}_{N \leftarrow H}\right]\left[\boldsymbol{T}_{H \leftarrow G}\right]_{G}^{H} \overrightarrow{\boldsymbol{\theta}}^{G}+\left[\boldsymbol{T}_{N \leftarrow H}\right]\left[\boldsymbol{T}_{H \leftarrow G}\right]\left[\boldsymbol{T}_{G \leftarrow n_{j}}\right]_{n_{j}}^{G} \overrightarrow{\boldsymbol{\theta}}^{n_{j}} \\
& =[\boldsymbol{I}]_{1 \times 1} \stackrel{N}{\boldsymbol{\theta}^{H}}+[\boldsymbol{I}]_{1 \times 1}[\boldsymbol{I}]_{1 \times 1} \underset{G}{H} \overrightarrow{\boldsymbol{\theta}}^{G}+[\boldsymbol{I}]_{1 \times 1}[\boldsymbol{I}]_{1 \times 1}[\boldsymbol{I}]_{1 \times 1} \underset{n_{j}}{G} \overrightarrow{\boldsymbol{\theta}}^{n_{j}} \\
& ={ }_{H}^{N} \overrightarrow{\boldsymbol{\theta}}^{H}+{ }_{G}^{H} \overrightarrow{\boldsymbol{\theta}}^{G}+{ }_{n_{j}}^{G} \overrightarrow{\boldsymbol{\theta}}^{n_{j}}
\end{aligned}
$$

The $j$ th DFE node's angular velocity is obtained by differentiating Equation 3.15:

$$
\begin{aligned}
{ }_{N}^{N} \overrightarrow{\boldsymbol{\omega}}^{n_{j}}= & \frac{\mathrm{d}}{\mathrm{d} t}\left({ }_{N}^{N} \overrightarrow{\boldsymbol{\theta}}^{n_{j}}\right) \\
= & \frac{\mathrm{d}}{\mathrm{d} t}\left(\left[\boldsymbol{T}_{N \leftarrow H}\right]_{H}^{N} \overrightarrow{\boldsymbol{\theta}}^{H}\right)+\frac{\mathrm{d}}{\mathrm{d} t}\left(\left[\boldsymbol{T}_{N \leftarrow H}\right]\left[\boldsymbol{T}_{H \leftarrow G}\right]_{G}^{H} \overrightarrow{\boldsymbol{\theta}}^{G}\right) \\
& +\frac{\mathrm{d}}{\mathrm{d} t}\left(\left[\boldsymbol{T}_{N \leftarrow H}\right]\left[\boldsymbol{T}_{H \leftarrow G}\right]\left[\boldsymbol{T}_{G \leftarrow n_{j}}\right]_{n_{j}}^{G} \overrightarrow{\boldsymbol{\theta}}^{n_{j}}\right) \\
= & {\left[\boldsymbol{T}_{N \leftarrow H}\right]_{H}^{N} \dot{\overrightarrow{\boldsymbol{\theta}}}^{H}+\left[\boldsymbol{T}_{N \leftarrow H}\right]\left[\boldsymbol{T}_{H \leftarrow G}\right]_{G}^{H} \dot{\overrightarrow{\boldsymbol{\theta}}}^{G}+\left[\boldsymbol{T}_{N \leftarrow H}\right]\left[\boldsymbol{T}_{H \leftarrow G}\right]\left[\boldsymbol{T}_{G \leftarrow n_{j}}\right]_{n_{j}}^{G} \dot{\overrightarrow{\boldsymbol{\theta}}}^{n_{j}} } \\
= & {\left[\boldsymbol{T}_{N \leftarrow H}\right]_{H}^{N} \overrightarrow{\boldsymbol{\omega}}^{H}+\left[\boldsymbol{T}_{N \leftarrow H}\right]\left[\boldsymbol{T}_{H \leftarrow G}\right]_{G}^{H} \overrightarrow{\boldsymbol{\omega}}^{G}+\left[\boldsymbol{T}_{N \leftarrow H}\right]\left[\boldsymbol{T}_{H \leftarrow G}\right]\left[\boldsymbol{T}_{G \leftarrow n_{j}}\right]_{n_{j}}^{G} \overrightarrow{\boldsymbol{\omega}}^{n_{j}} } \\
= & {[\boldsymbol{I}]_{1 \times 1}{ }_{H}^{N} \overrightarrow{\boldsymbol{\omega}}^{H}+[\boldsymbol{I}]_{1 \times 1}{ }_{G}^{H} \overrightarrow{\boldsymbol{\omega}}^{G}+[\boldsymbol{I}]_{1 \times 1}{ }_{n_{j}}^{G} \overrightarrow{\boldsymbol{\omega}}^{n_{j}} } \\
= & { }_{H}^{N} \overrightarrow{\boldsymbol{\omega}}^{H}+{ }_{G}^{H} \overrightarrow{\boldsymbol{\omega}}^{G}+{ }_{n_{j}}^{G} \overrightarrow{\boldsymbol{\omega}}^{n_{j}}
\end{aligned}
$$

Given that the transformation matrices are identity, the equivalence ${ }_{N}^{N} \overrightarrow{\boldsymbol{\omega}}^{n_{j}}={ }_{n_{j}}^{N} \overrightarrow{\boldsymbol{\omega}}^{n_{j}}$ holds. Therefore, expressing Equation 3.16 in the $j$ th DFE node coordinate system becomes

$$
{ }_{n_{j}}^{N} \overrightarrow{\boldsymbol{\omega}}^{n_{j}}={ }_{N}^{N} \overrightarrow{\boldsymbol{\omega}}^{n_{j}}={ }_{H}^{N} \overrightarrow{\boldsymbol{\omega}}^{H}+{ }_{G}^{H} \overrightarrow{\boldsymbol{\omega}}^{G}+{ }_{n_{j}}^{G} \overrightarrow{\boldsymbol{\omega}}^{n_{j}}
$$


The $j$ th DFE node's angular acceleration is obtained by differentiating Equation 3.16:

$$
\begin{aligned}
& { }_{N}^{N} \overrightarrow{\boldsymbol{\alpha}}^{n_{j}}=\frac{\mathrm{d}}{\mathrm{d} t}\left({ }_{N}^{N} \overrightarrow{\boldsymbol{\omega}}^{n_{j}}\right) \\
& =\frac{\mathrm{d}}{\mathrm{d} t}\left(\left[\boldsymbol{T}_{N \leftarrow H}\right]_{H}^{N} \overrightarrow{\boldsymbol{\omega}}^{H}\right)+\frac{\mathrm{d}}{\mathrm{d} t}\left(\left[\boldsymbol{T}_{N \leftarrow H}\right]\left[\boldsymbol{T}_{H \leftarrow G}\right]_{G}^{H} \overrightarrow{\boldsymbol{\omega}}^{G}\right) \\
& +\frac{\mathrm{d}}{\mathrm{d} t}\left(\left[\boldsymbol{T}_{N \leftarrow H}\right]\left[\boldsymbol{T}_{H \leftarrow G}\right]\left[\boldsymbol{T}_{G \leftarrow n_{j}}\right]_{n_{j}}^{G} \overrightarrow{\boldsymbol{\omega}}^{n_{j}}\right) \\
& =\left[\dot{\boldsymbol{T}}_{N \leftarrow H}\right]_{H}^{N} \overrightarrow{\boldsymbol{\omega}}^{H}+\left[\boldsymbol{T}_{N \leftarrow H}\right]_{H}^{N} \dot{\overrightarrow{\boldsymbol{\omega}}}^{H}+\left[\dot{\boldsymbol{T}}_{N \leftarrow H}\right]\left[\boldsymbol{T}_{H \leftarrow G}\right]_{G}^{H} \overrightarrow{\boldsymbol{\omega}}^{G} \\
& +\left[\boldsymbol{T}_{N \leftarrow H}\right]\left[\dot{\boldsymbol{T}}_{H \leftarrow G}\right]_{G}^{H} \overrightarrow{\boldsymbol{\omega}}^{G}+\left[\boldsymbol{T}_{N \leftarrow H}\right]\left[\boldsymbol{T}_{H \leftarrow G}\right]_{G}^{H} \dot{\overrightarrow{\boldsymbol{\omega}}}^{G} \\
& +\left[\dot{\boldsymbol{T}}_{N \leftarrow H}\right]\left[\boldsymbol{T}_{H \leftarrow G}\right]\left[\boldsymbol{T}_{G \leftarrow n_{j}}\right]_{n_{j}}^{G} \overrightarrow{\boldsymbol{\omega}}^{n_{j}}+\left[\boldsymbol{T}_{N \leftarrow H}\right]\left[\dot{\boldsymbol{T}}_{H \leftarrow G}\right]\left[\boldsymbol{T}_{G \leftarrow n_{j}}\right]_{n_{j}}^{G} \overrightarrow{\boldsymbol{\omega}}^{n_{j}} \\
& +\left[\boldsymbol{T}_{N \leftarrow H}\right]\left[\boldsymbol{T}_{H \leftarrow G}\right]\left[\dot{\boldsymbol{T}}_{G \leftarrow n_{j}}\right]_{n_{j}}^{G} \overrightarrow{\boldsymbol{\omega}}^{n_{j}}+\left[\boldsymbol{T}_{N \leftarrow H}\right]\left[\boldsymbol{T}_{H \leftarrow G}\right]\left[\boldsymbol{T}_{G \leftarrow n_{j}}\right]_{n_{j}}^{G} \dot{\overrightarrow{\boldsymbol{\omega}}}^{n_{j}}
\end{aligned}
$$

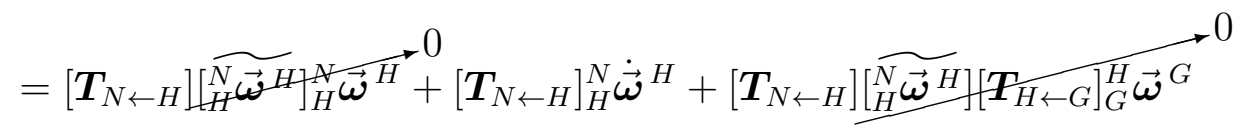

$$
\begin{aligned}
& +\left[\boldsymbol{T}_{N \leftarrow H}\right]\left[\boldsymbol{T}_{H \leftarrow G}\right]\left[\widetilde{{ }_{G}^{H} \overrightarrow{\boldsymbol{\omega}} G}\right]_{G}^{H} \overrightarrow{\boldsymbol{\omega}} \vec{C}^{0}+\left[\boldsymbol{T}_{N \leftarrow H}\right]\left[\boldsymbol{T}_{H \leftarrow G}\right]_{G}^{H} \dot{\overrightarrow{\boldsymbol{\omega}}}^{G}
\end{aligned}
$$

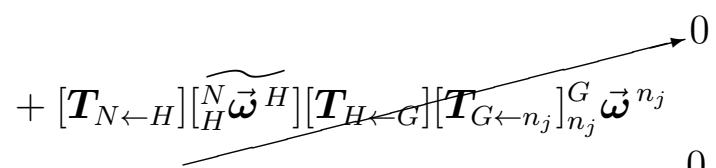

$$
\begin{aligned}
& \left.+\left[\boldsymbol{T}_{N \leftarrow H}\right]\left[\boldsymbol{T}_{H \leftarrow G}\right] \widetilde{\left[{ }_{G}^{H} \overrightarrow{\boldsymbol{\omega}} G\right.}\right]\left[\boldsymbol{T}_{G \leftarrow n_{j}}\right]_{n_{j}}^{G} \overrightarrow{\boldsymbol{\omega}}^{n_{j}} 0 \\
& +\left[\boldsymbol{T}_{N \leftarrow H}\right]\left[\boldsymbol{T}_{H \leftarrow G}\right]\left[\boldsymbol{T}_{G \leftarrow n_{j}}\right]\left[\widetilde{\left[\vec{G}_{j} \overrightarrow{\boldsymbol{\omega}}_{n_{j}}\right]_{n_{j}} \overrightarrow{\boldsymbol{\omega}}^{n_{j}}} 0\right. \\
& +\left[\boldsymbol{T}_{N \leftarrow H}\right]\left[\boldsymbol{T}_{H \leftarrow G}\right]\left[\boldsymbol{T}_{G \leftarrow n_{j}}\right]_{n_{j}}^{G} \dot{\overrightarrow{\boldsymbol{\omega}}}^{n_{j}} \\
& =\left[\boldsymbol{T}_{N \leftarrow H}\right]_{H}^{N} \dot{\overrightarrow{\boldsymbol{\omega}}}^{H}+\left[\boldsymbol{T}_{N \leftarrow H}\right]\left[\boldsymbol{T}_{H \leftarrow G}\right]_{G}^{H} \dot{\overrightarrow{\boldsymbol{\omega}}}^{G}+\left[\boldsymbol{T}_{N \leftarrow H}\right]\left[\boldsymbol{T}_{H \leftarrow G}\right]\left[\boldsymbol{T}_{G \leftarrow n_{j}}\right]_{n_{j}}^{G} \dot{\overrightarrow{\boldsymbol{\omega}}}^{n_{j}} \\
& =[\boldsymbol{I}]_{1 \times 1} \underset{H}{N} \dot{\overrightarrow{\boldsymbol{\omega}}}^{H}+[\boldsymbol{I}]_{1 \times 1} \underset{G}{H} \dot{\overrightarrow{\boldsymbol{\omega}}}^{G}+[\boldsymbol{I}]_{1 \times 1} \underset{n_{j}}{G} \dot{\overrightarrow{\boldsymbol{\omega}}}^{n_{j}} \\
& ={ }_{H}^{N} \dot{\overrightarrow{\boldsymbol{\omega}}}^{H}+{ }_{G}^{H} \dot{\overrightarrow{\boldsymbol{\omega}}}^{G}+{ }_{n_{j}}^{G} \dot{\overrightarrow{\boldsymbol{\omega}}}^{n_{j}}
\end{aligned}
$$

Since the transformation matrices are identity, terms which contain transformation time-derivatives yield skew angular velocities. The cross-product $[\widetilde{\overrightarrow{\boldsymbol{\omega}}}] \overrightarrow{\boldsymbol{\omega}} \equiv 0$ eliminates these terms. Similarly to Equation 3.16, the equivalence ${ }_{N}^{N} \overrightarrow{\boldsymbol{\alpha}}^{n_{j}}={ }_{n_{j}}^{N} \overrightarrow{\boldsymbol{\alpha}}^{n_{j}}$ holds. Therefore, expressing Equation 3.18 in the $j$ th DFE node coordinate system becomes

$$
{ }_{n_{j}}^{N} \overrightarrow{\boldsymbol{\alpha}}^{n_{j}}={ }_{N}^{N} \overrightarrow{\boldsymbol{\alpha}}^{n_{j}}={ }_{H}^{N} \dot{\overrightarrow{\boldsymbol{\omega}}}^{H}+{ }_{G}^{H} \dot{\overrightarrow{\boldsymbol{\omega}}}^{G}+{ }_{n_{j}}^{G} \dot{\overrightarrow{\boldsymbol{\omega}}}^{n_{j}}
$$

At this point, The derivations of the kinematic expressions are complete for the bodies studied for dynamic response.

The equations for velocity and acceleration can be expressed in the form of Equations 2.15 through 2.18 where the kinematic equations are compactly expressed in 
terms of the vector of generalized speeds $\boldsymbol{u}$. Linear velocities are compactly expressed

$$
\begin{aligned}
{ }_{k}^{N} \overrightarrow{\boldsymbol{v}}^{k} & =\left[{ }_{k}^{N} \boldsymbol{V}^{k}\right] \boldsymbol{u} \\
& =\left[\begin{array}{lll}
{ }_{k} \boldsymbol{V}^{u_{1}} & \ldots & { }_{k} \boldsymbol{V}^{u_{N_{g c}}}
\end{array}\right]_{2 \times N_{g c}}\left\{\begin{array}{c}
u_{1} \\
\vdots \\
u_{N_{g c}}
\end{array}\right\}_{N_{g c} \times 1}
\end{aligned}
$$

and angular velocities are compactly expressed

$$
\begin{aligned}
{ }_{k}^{N} \overrightarrow{\boldsymbol{\omega}}^{k} & =\left[\begin{array}{l}
{ }_{k}^{N} \boldsymbol{W}^{k}
\end{array}\right] \boldsymbol{u} \\
& =\left[\begin{array}{lll}
{ }_{k} \boldsymbol{W}^{u_{1}} & \ldots & { }_{k} \boldsymbol{W}^{u_{N g c}}
\end{array}\right]_{1 \times N_{g c}}\left\{\begin{array}{c}
u_{1} \\
\vdots \\
u_{N_{g c}}
\end{array}\right\}_{N_{g c} \times 1}
\end{aligned}
$$

where by inspection of Equation 3.20, the linear and angular partial velocity matrices $\left[{ }_{k}^{N} \boldsymbol{V}^{k}\right]$ and $\left[{ }_{k}^{N} \boldsymbol{W}^{k}\right]$ are the arrays of coefficients for each of the generalized speed terms in the kinematic expressions. Each element $\boldsymbol{V}$ and $\boldsymbol{W}$ represent the linear and angular partial contributions along $u_{i}$ to kinematic quantity of body $k$, expressed in the $k$ th frame. Otherwise stated, it transforms the expression of the kinematic quantity in terms of generalized quantities to body quantities expressed in the $k$ th body's coordinate system.

Similarly, the equations of linear and angular accelerations can be expressed in terms of the system generalized accelerations $\dot{\boldsymbol{u}}$ as

$$
\begin{aligned}
{ }_{k}^{N} \overrightarrow{\boldsymbol{a}}^{k} & =\left[{ }_{k}^{N} \boldsymbol{V}^{k}\right] \dot{\boldsymbol{u}}+\overrightarrow{\boldsymbol{Z}}_{k} \\
& =\left[\begin{array}{lll}
{ }_{k} \boldsymbol{V}^{u_{1}} & \cdots & { }_{k} \boldsymbol{V}^{u_{N_{g c}}}
\end{array}\right]_{2 \times N_{g c}}\left\{\begin{array}{c}
\dot{u}_{1} \\
\vdots \\
\dot{u}_{N_{g c}}
\end{array}\right\}_{N_{g c} \times 1}+\overrightarrow{\boldsymbol{Z}}_{k}
\end{aligned}
$$

and

$$
\begin{aligned}
{ }_{k}^{N} \overrightarrow{\boldsymbol{\alpha}}^{k} & =\left[{ }_{k}^{N} \boldsymbol{W}^{k}\right] \dot{\boldsymbol{u}}+\overrightarrow{\boldsymbol{Y}}_{k} \\
& =\left[\begin{array}{lll}
{ }_{k} \boldsymbol{W}^{u_{1}} & \ldots & { }_{k} \boldsymbol{W}^{u_{N_{g c}}}
\end{array}\right]_{1 \times N_{g c}}\left\{\begin{array}{c}
\dot{u}_{1} \\
\vdots \\
\dot{u}_{N_{g c}}
\end{array}\right\}_{N_{g c} \times 1}+\overrightarrow{\boldsymbol{Y}}_{k}
\end{aligned}
$$

In the case of acceleration expressions, the terms which do not contain generalized accelerations expressed in vector form are grouped into the remainder acceleration 
terms $\overrightarrow{\boldsymbol{Z}}_{k}$ and, $\overrightarrow{\boldsymbol{Y}}_{k}$ respectively. Since $\dot{\boldsymbol{u}}$ is the locally evaluated derivative of $\boldsymbol{u}$, the linear and angular partial velocity matrices are identical for velocity and acceleration expressions for a given body.

As shown in Section 2.2, the partial velocity matrices are integral components of Kane's method. As such, for the aircraft's airframe, the translational partial velocity matrix $\left[{ }_{H}^{N} \boldsymbol{V}^{H}\right]$ is obtained by inspection of Equation 3.3, and the angular partial velocity matrix $\left[{ }_{H}^{N} \boldsymbol{W}^{H}\right]$ by inspection of Equation 3.13 :

$$
\begin{aligned}
& {\left[{ }_{H}^{N} \boldsymbol{V}^{H}\right]=\left[\begin{array}{llll}
{[0]_{2 \times 12}} & {\left[\boldsymbol{T}_{H \leftarrow N}\right]} & {[0]_{2 \times 1}} & {[0]_{2 \times\left(N_{g c}-15\right)}}
\end{array}\right]} \\
& \left.\left[{ }_{H}^{N} \boldsymbol{W}^{H}\right]=\left[[0]_{1 \times 12} \quad 0 \quad 00110\right]_{1 \times\left(N_{g c}-15\right)}\right]
\end{aligned}
$$

For the general case of the $j$ th DFE node, the translational partial velocity matrix $\left[{ }_{n}^{N} \boldsymbol{V}^{n_{j}}\right]$ is obtained by inspection of Equation 3.9, and the angular partial velocity matrix $\left[{ }_{n_{j}}^{N} \boldsymbol{W}^{n_{j}}\right]$ by inspection of Equation 3.16:

$$
\begin{aligned}
& {\left[\begin{array}{c}
{ }_{n_{j}}^{N} \boldsymbol{V}^{n_{j}}
\end{array}\right]=\left[[0]_{2 \times 12} \quad\left[\boldsymbol{T}_{n_{j} \leftarrow N}\right]-\left[\boldsymbol{T}_{n_{j} \leftarrow H}\right]\left\{\widetilde{\left[{ }_{H} \overrightarrow{\boldsymbol{r}} G\right.}\right]+\left[\widetilde{\left.\boldsymbol{T}_{H \leftarrow G}\right]_{G}^{G}} \overrightarrow{\boldsymbol{r}}^{n_{j}}\right\}\right.} \\
& {\left[\boldsymbol{T}_{n_{j} \leftarrow H}\right] \quad-\left[\boldsymbol{T}_{n_{j} \leftarrow G}\right]\left[\widetilde{\left[{ }_{G}^{G} \overrightarrow{\boldsymbol{r}}^{n_{j}}\right.}\right] \quad[0]_{2 \times 3(j-1)} \quad\left[\boldsymbol{T}_{n_{j} \leftarrow G}\right]} \\
& \left.[0]_{2 \times 1}[0]_{2 \times(i-3 j-18)}\right]
\end{aligned}
$$

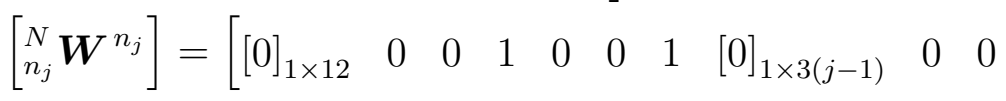

$$
\begin{aligned}
& \left.1 \quad[0]_{1 \times\left(N_{g c}-18-3 j\right)}\right]
\end{aligned}
$$

Recalling that there are $N_{g c}$ generalized coordinates in the system.

The linear remainder accelerations $\overrightarrow{\boldsymbol{Z}}$ and angular remainder accelerations $\overrightarrow{\boldsymbol{Y}}$ are

$$
\begin{aligned}
& \vec{Z}_{H}=0
\end{aligned}
$$

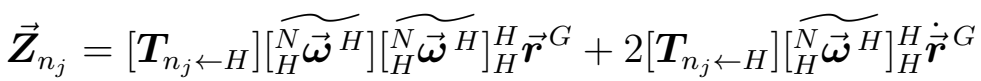

$$
\begin{aligned}
& +\left[\boldsymbol{T}_{n_{j} \leftarrow H}\right]\left[\widetilde{N_{H}^{N \overrightarrow{\boldsymbol{\omega}} H}}\right]\left[\widetilde{N_{H} \overrightarrow{\boldsymbol{\omega}} H}\right]\left[\boldsymbol{T}_{H \leftarrow G}\right]_{G}^{G} \overrightarrow{\boldsymbol{r}}^{n_{j}} \\
& +2\left[\boldsymbol{T}_{n_{j} \leftarrow H}\right]\left[\widetilde{{ }_{H}^{N} \overrightarrow{\boldsymbol{\omega}}^{H}}\right]\left[\boldsymbol{T}_{H \leftarrow G}\right]\left[\widetilde{H_{G}^{H} \overrightarrow{\boldsymbol{\omega}}^{G}}\right]_{G}^{G} \overrightarrow{\boldsymbol{r}}^{n_{j}} \\
& \left.+2\left[\boldsymbol{T}_{n_{j} \leftarrow H}\right]\left[\widetilde{{ }_{H}^{N} \overrightarrow{\boldsymbol{\omega}}^{H}}\right]\left[\boldsymbol{T}_{H \leftarrow G}\right]_{G}^{G} \dot{\overrightarrow{\boldsymbol{r}}}^{n_{j}}+\left[\boldsymbol{T}_{n_{j} \leftarrow G}\right] \widetilde{{ }_{G}^{H} \overrightarrow{\boldsymbol{\omega}}^{G}}\right]\left[\widetilde{H_{G}^{H} \overrightarrow{\boldsymbol{\omega}}^{G}}\right]_{G}^{G} \overrightarrow{\boldsymbol{r}}^{n_{j}} \\
& +2\left[\boldsymbol{T}_{n_{j} \leftarrow G}\right]\left[\widetilde{{ }_{G}^{H} \overrightarrow{\boldsymbol{\omega}} G}\right]_{G}^{G} \dot{\overrightarrow{\boldsymbol{r}}} n_{j} \\
& \overrightarrow{\boldsymbol{Y}}_{H}=0 \\
& \overrightarrow{\boldsymbol{Y}}_{n_{j}}=0
\end{aligned}
$$


Thus the complete velocities and accelerations of the studied bodies can be expressed

$$
\begin{aligned}
{ }_{H}^{N} \overrightarrow{\boldsymbol{v}}^{H} & =\left[{ }_{H}^{N} \boldsymbol{V}^{H}\right] \overrightarrow{\boldsymbol{u}} \\
{ }_{H}^{N} \overrightarrow{\boldsymbol{\omega}}^{H} & =\left[{ }_{H}^{N} \boldsymbol{W}^{H}\right] \overrightarrow{\boldsymbol{u}} \\
{ }_{H}^{N} \overrightarrow{\boldsymbol{a}}^{H} & =\left[{ }_{H}^{N} \boldsymbol{V}^{H}\right] \dot{\overrightarrow{\boldsymbol{u}}}+\overrightarrow{\boldsymbol{Z}}_{H} \\
{ }_{H}^{N} \overrightarrow{\boldsymbol{\alpha}}^{H} & =\left[{ }_{H}^{N} \boldsymbol{W}^{H}\right] \dot{\overrightarrow{\boldsymbol{u}}}+\overrightarrow{\boldsymbol{Y}}_{H}
\end{aligned}
$$

for the airframe, and

$$
\begin{aligned}
& { }_{n_{j}}^{N} \overrightarrow{\boldsymbol{v}}^{n_{j}}=\left[\begin{array}{l}
N \\
{ }_{n_{j}}
\end{array} \boldsymbol{V}^{n_{j}}\right] \overrightarrow{\boldsymbol{u}} \\
& { }_{n_{j}}^{N} \overrightarrow{\boldsymbol{\omega}}^{n_{j}}=\left[{ }_{n_{j}}^{N} \boldsymbol{W}^{n_{j}}\right] \overrightarrow{\boldsymbol{u}} \\
& { }_{n_{j}}^{N} \overrightarrow{\boldsymbol{a}}^{n_{j}}=\left[\begin{array}{l}
N \\
{ }_{n}
\end{array} \boldsymbol{V}^{n_{j}}\right] \dot{\overrightarrow{\boldsymbol{u}}}+\overrightarrow{\boldsymbol{Z}}_{n_{j}} \\
& { }_{n_{j}}^{N} \overrightarrow{\boldsymbol{\alpha}}^{n_{j}}=\left[{ }_{n_{j}}^{N} \boldsymbol{W}^{n_{j}}\right] \dot{\overrightarrow{\boldsymbol{u}}}+\overrightarrow{\boldsymbol{Y}}_{n_{j}}
\end{aligned}
$$

for the DFE nodes. Kinematic expressions for the studied bodies which compose the complete aircraft, have been derived. Nevertheless, the positions and velocities of the vertices defining each polygon in the simulation must be determined. This will enable proper handling of collision detection and oriented dynamic response.

\subsection{Polygon Kinematics}

In the SRAMSS environment, the dynamic interface formed between the aircraft and ship is governed by the interaction of the aircraft with defined polygons. In effect, this interaction is the appropriately-oriented dynamic response generated by the contact dynamics model. The dynamic interface interactions comprise aircraft response to ship motion, RSD deck manoeuvring, and RSD securing.

The polygons included in the SRAMSS environment have their vertices defined with respect to their own frame of reference. This allow the prescription of motion for the polygon while maintaining a single general equation defining each polygon vertex. For this work, only the positions and velocities of the vertices are required. Even so, the partial velocity matrices include the generalized speeds for these polygons, such that the partial velocity matrices can be determined for these polygons in the case where dynamic responses of these polygons are studied. This requires that the

equations of motion be expressed relative to the inertial frame. Expression relative to this frame also facilitates calculation of relative velocities and dynamic quantities, 
since the inertial frame is common to both the aircraft's and the polygon's chain of frames.

The position of the $m$ th ship deck polygon vertex $S_{m}$ with respect to the inertial frame, expressed in the inertial frame, is

$$
\begin{aligned}
{ }_{N}^{N} \overrightarrow{\boldsymbol{r}}^{S_{m}} & ={ }_{N}^{N} \overrightarrow{\boldsymbol{r}}^{S}+{ }_{N}^{S} \overrightarrow{\boldsymbol{r}}^{S_{m}} \\
& ={ }_{N}^{N} \boldsymbol{\boldsymbol { r }}^{S}+\left[\boldsymbol{T}_{N \leftarrow S}\right]_{S}^{S} \overrightarrow{\boldsymbol{r}}^{S_{m}}
\end{aligned}
$$

The $m$ th ship deck vertex velocity is obtained by differentiating Equation 3.40, resulting in

$$
\begin{aligned}
& { }_{N}^{N} \overrightarrow{\boldsymbol{v}}^{S_{m}}=\frac{\mathrm{d}}{\mathrm{d} t}\left({ }_{N}^{N} \overrightarrow{\boldsymbol{r}}^{S_{m}}\right) \\
& =\frac{\mathrm{d}}{\mathrm{d} t}\left({ }_{N}^{N} \overrightarrow{\boldsymbol{r}}^{S}\right)+\frac{\mathrm{d}}{\mathrm{d} t}\left(\left[\boldsymbol{T}_{N \leftarrow S}\right]_{S}^{S} \overrightarrow{\boldsymbol{r}}^{S_{m}}\right) \\
& ={ }_{N}^{N} \dot{\overrightarrow{\boldsymbol{r}}}^{S}+\left[\dot{\boldsymbol{T}}_{N \leftarrow S}\right]_{S}^{S} \overrightarrow{\boldsymbol{r}}^{S_{m}}+\left[\boldsymbol{T}_{N \leftarrow S}\right]_{S}^{S} \dot{\overrightarrow{\boldsymbol{r}}}^{S_{m}} \\
& \left.={ }_{N}^{N} \dot{\overrightarrow{\boldsymbol{r}}} S+\left[\boldsymbol{T}_{N \leftarrow S}\right] \widetilde{\int_{S}^{N} \overrightarrow{\boldsymbol{\omega}} S}\right]_{S}^{S} \overrightarrow{\boldsymbol{r}}^{S_{m}}+\left[\boldsymbol{T}_{N \leftarrow S}\right]_{S}^{S} \dot{\overrightarrow{\boldsymbol{p}}} S_{m}^{-} \\
& ={ }_{N}^{N} \dot{\overrightarrow{\boldsymbol{r}}}^{S}+\left[\boldsymbol{T}_{N \leftarrow S}\right]\left[\widetilde{{ }_{S}^{N} \overrightarrow{\boldsymbol{\omega}} S}\right]_{S}^{S} \overrightarrow{\boldsymbol{r}}^{S_{m}}
\end{aligned}
$$

Next along the polygon chain of frames is the RSD deck. The position of the $m$ th RSD deck polygon vertex $D_{m}$ with respect to the inertial frame, expressed in the inertial frame, is

$$
\begin{aligned}
{ }_{N}^{N} \overrightarrow{\boldsymbol{r}}^{D_{m}} & ={ }_{N}^{N} \overrightarrow{\boldsymbol{r}}^{S}+{ }_{N}^{S} \overrightarrow{\boldsymbol{r}}^{D}+{ }_{N}^{D} \overrightarrow{\boldsymbol{r}}^{D_{m}} \\
& ={ }_{N}^{N} \overrightarrow{\boldsymbol{r}}^{S}+\left[\boldsymbol{T}_{N \leftarrow S}\right]_{S}^{S} \overrightarrow{\boldsymbol{r}}^{D}+\left[\boldsymbol{T}_{N \leftarrow S}\right]\left[\boldsymbol{T}_{S \leftarrow D}\right]_{D}^{D} \overrightarrow{\boldsymbol{r}}^{D_{m}} \\
& ={ }_{N}^{N} \overrightarrow{\boldsymbol{r}}^{S}+\left[\boldsymbol{T}_{N \leftarrow S}\right]_{S}^{S} \overrightarrow{\boldsymbol{r}}^{D}+\left[\boldsymbol{T}_{N \leftarrow D}\right]_{D}^{D} \overrightarrow{\boldsymbol{r}}^{D_{m}}
\end{aligned}
$$

The $m$ th RSD deck vertex velocity is obtained by differentiating Equation 3.42, re- 
sulting in

$$
\begin{aligned}
& { }_{N}^{N} \overrightarrow{\boldsymbol{v}}^{D_{m}}=\frac{\mathrm{d}}{\mathrm{d} t}\left({ }_{N}^{N} \overrightarrow{\boldsymbol{r}}^{D_{m}}\right) \\
& =\frac{\mathrm{d}}{\mathrm{d} t}\left({ }_{N}^{N} \overrightarrow{\boldsymbol{r}}^{S}\right)+\frac{\mathrm{d}}{\mathrm{d} t}\left(\left[\boldsymbol{T}_{N \leftarrow S}\right]_{S}^{S} \overrightarrow{\boldsymbol{r}}^{D}\right)+\frac{\mathrm{d}}{\mathrm{d} t}\left(\left[\boldsymbol{T}_{N \leftarrow S}\right]\left[\boldsymbol{T}_{S \leftarrow D}\right]_{D}^{D} \overrightarrow{\boldsymbol{r}}^{D_{m}}\right) \\
& ={ }_{N}^{N} \dot{\overrightarrow{\boldsymbol{r}}}^{S}+\left[\dot{\boldsymbol{T}}_{N \leftarrow S}\right]_{S}^{S} \overrightarrow{\boldsymbol{r}}^{D}+\left[\boldsymbol{T}_{N \leftarrow S}\right]_{S}^{S} \dot{\overrightarrow{\boldsymbol{r}}}^{D} \\
& +\left[\dot{\boldsymbol{T}}_{N \leftarrow S}\right]\left[\boldsymbol{T}_{S \leftarrow D}\right]_{D}^{D} \overrightarrow{\boldsymbol{r}}^{D_{m}}+\left[\boldsymbol{T}_{N \leftarrow S}\right]\left[\dot{\boldsymbol{T}}_{S \leftarrow D}\right]_{D}^{D} \overrightarrow{\boldsymbol{r}}^{D_{m}} \\
& +\left[\boldsymbol{T}_{N \leftarrow S}\right]\left[\boldsymbol{T}_{S \leftarrow D}\right]_{D}^{D} \dot{\overrightarrow{\boldsymbol{r}}}^{D_{m}} \\
& \left.={ }_{N}^{N} \dot{\overrightarrow{\boldsymbol{r}}}^{S}+\left[\boldsymbol{T}_{N \leftarrow S}\right] \widetilde{{ }_{S}^{N} \overrightarrow{\boldsymbol{\omega}} S}\right]_{S}^{S} \overrightarrow{\boldsymbol{r}}^{D}+\left[\boldsymbol{T}_{N \leftarrow S}\right]_{S}^{S} \dot{\overrightarrow{\boldsymbol{r}}}^{D} \\
& +\left[\boldsymbol{T}_{N \leftarrow S}\right]\left[\widetilde{N_{S}^{N} \overrightarrow{\boldsymbol{\omega}} S}\right]\left[\boldsymbol{T}_{S \leftarrow D}\right]_{D}^{D} \overrightarrow{\boldsymbol{r}}^{D_{m}}+\left[\boldsymbol{T}_{N \leftarrow S}\right]\left[\boldsymbol{T}_{S \leftarrow D}\right]\left[\widetilde{{ }_{D}^{S} \overrightarrow{\boldsymbol{\omega}}^{D}}\right]_{D}^{D} \overrightarrow{\boldsymbol{r}}^{D_{m}} \\
& +\left[\boldsymbol{T}_{N \leftarrow S}\right]\left[\boldsymbol{T}_{S \leftarrow D}\right]_{D}^{D} \dot{\overrightarrow{\boldsymbol{p}}} D_{m}^{-} 0 \\
& ={ }_{N}^{N} \dot{\overrightarrow{\boldsymbol{r}}}^{S}+\left[\boldsymbol{T}_{N \leftarrow S}\right]\left[\widetilde{{ }_{S}^{N} \overrightarrow{\boldsymbol{\omega}} S}\right]_{S}^{S} \overrightarrow{\boldsymbol{r}}^{D}+\left[\boldsymbol{T}_{N \leftarrow S}\right]_{S}^{S} \dot{\overrightarrow{\boldsymbol{r}}}^{D} \\
& +\left[\boldsymbol{T}_{N \leftarrow S}\right]\left[\widetilde{{ }_{S}^{N} \overrightarrow{\boldsymbol{\omega}}^{S}}\right]\left[\boldsymbol{T}_{S \leftarrow D}\right]_{D}^{D} \overrightarrow{\boldsymbol{r}}^{D_{m}}+\left[\boldsymbol{T}_{N \leftarrow D}\right]\left[\widetilde{{ }_{D}^{S} \overrightarrow{\boldsymbol{\omega}}^{D}}\right]_{D}^{D} \overrightarrow{\boldsymbol{r}}^{D_{m}}
\end{aligned}
$$

Next along the polygon chain of frames are the wedge clamps. The position of the $m$ th left and right wedge clamp polygon vertices $C L_{m}$ and $C R_{m}$ with respect to the inertial frame, expressed in the inertial frame, are

$$
\begin{aligned}
{ }_{N}^{N} \overrightarrow{\boldsymbol{r}}^{C L_{m}}= & { }_{N}^{N} \overrightarrow{\boldsymbol{r}}^{S}+{ }_{N}^{S} \overrightarrow{\boldsymbol{r}}^{D}+{ }_{N}^{D} \overrightarrow{\boldsymbol{r}}^{C L}+{ }_{N}^{C L} \overrightarrow{\boldsymbol{r}}^{C L_{m}} \\
= & { }_{N}^{N} \overrightarrow{\boldsymbol{r}}^{S}+\left[\boldsymbol{T}_{N \leftarrow S}\right]_{S}^{S} \overrightarrow{\boldsymbol{r}}^{D}+\left[\boldsymbol{T}_{N \leftarrow S}\right]\left[\boldsymbol{T}_{S \leftarrow D}\right]_{D}^{D} \overrightarrow{\boldsymbol{r}}^{C L} \\
& +\left[\boldsymbol{T}_{N \leftarrow S}\right]\left[\boldsymbol{T}_{S \leftarrow D}\right]\left[\boldsymbol{T}_{D \leftarrow C L}\right]_{C L}^{C L} \overrightarrow{\boldsymbol{r}}^{C L_{m}} \\
= & { }_{N}^{N} \overrightarrow{\boldsymbol{r}}^{S}+\left[\boldsymbol{T}_{N \leftarrow S}\right]_{S}^{S} \overrightarrow{\boldsymbol{r}}^{D}+\left[\boldsymbol{T}_{N \leftarrow D}\right]_{D}^{D} \overrightarrow{\boldsymbol{r}}^{C L}+\left[\boldsymbol{T}_{N \leftarrow C L}\right]_{C L}^{C L} \overrightarrow{\boldsymbol{r}}^{C L_{m}}
\end{aligned}
$$

and

$$
\begin{aligned}
{ }_{N}^{N} \overrightarrow{\boldsymbol{r}}^{C R_{m}}= & { }_{N}^{N} \overrightarrow{\boldsymbol{r}}^{S}+{ }_{N}^{S} \overrightarrow{\boldsymbol{r}}^{D}+{ }_{N}^{D} \overrightarrow{\boldsymbol{r}}^{C R}+{ }_{N}^{C R} \overrightarrow{\boldsymbol{r}}^{C R_{m}} \\
= & { }_{N}^{N} \overrightarrow{\boldsymbol{r}}^{S}+\left[\boldsymbol{T}_{N \leftarrow S}\right]_{S}^{S} \overrightarrow{\boldsymbol{r}}^{D}+\left[\boldsymbol{T}_{N \leftarrow S}\right]\left[\boldsymbol{T}_{S \leftarrow D}\right]_{D}^{D} \overrightarrow{\boldsymbol{r}}^{C R} \\
& +\left[\boldsymbol{T}_{N \leftarrow S}\right]\left[\boldsymbol{T}_{S \leftarrow D}\right]\left[\boldsymbol{T}_{D \leftarrow C R}\right]_{C R}^{C R} \overrightarrow{\boldsymbol{r}}^{C R_{m}} \\
= & { }_{N}^{N} \overrightarrow{\boldsymbol{r}}^{S}+\left[\boldsymbol{T}_{N \leftarrow S}\right]_{S}^{S} \overrightarrow{\boldsymbol{r}}^{D}+\left[\boldsymbol{T}_{N \leftarrow D}\right]_{D}^{D} \overrightarrow{\boldsymbol{r}}^{C R}+\left[\boldsymbol{T}_{N \leftarrow C R}\right]_{C R}^{C R} \overrightarrow{\boldsymbol{r}}^{C R_{m}}
\end{aligned}
$$

The $m$ th left and right wedge clamp polygon vertex velocities are obtained by differ- 
entiating Equations 3.44 and 3.45, resulting in

$$
\begin{aligned}
& { }_{N}^{N} \overrightarrow{\boldsymbol{v}}^{C L_{m}}=\frac{\mathrm{d}}{\mathrm{d} t}\left({ }_{N}^{N} \overrightarrow{\boldsymbol{r}}^{C L_{m}}\right) \\
& =\frac{\mathrm{d}}{\mathrm{d} t}\left({ }_{N}^{N} \boldsymbol{r}^{S}\right)+\frac{\mathrm{d}}{\mathrm{d} t}\left(\left[\boldsymbol{T}_{N \leftarrow S}\right]_{S}^{S} \overrightarrow{\boldsymbol{r}}^{D}\right)+\frac{\mathrm{d}}{\mathrm{d} t}\left(\left[\boldsymbol{T}_{N \leftarrow S}\right]\left[\boldsymbol{T}_{S \leftarrow D}\right]_{D}^{D} \overrightarrow{\boldsymbol{r}}^{C L}\right) \\
& +\frac{\mathrm{d}}{\mathrm{d} t}\left(\left[\boldsymbol{T}_{N \leftarrow S}\right]\left[\boldsymbol{T}_{S \leftarrow D}\right]\left[\boldsymbol{T}_{D \leftarrow C L}\right]_{C L}^{C L} \overrightarrow{\boldsymbol{r}}^{C L_{m}}\right) \\
& ={ }_{N}^{N} \dot{\overrightarrow{\boldsymbol{r}}}^{S}+\left[\dot{\boldsymbol{T}}_{N \leftarrow S}\right]_{S}^{S} \overrightarrow{\boldsymbol{r}}^{D}+\left[\boldsymbol{T}_{N \leftarrow S}\right]_{S}^{S} \dot{\overrightarrow{\boldsymbol{r}}}^{D}+\left[\dot{\boldsymbol{T}}_{N \leftarrow S}\right]\left[\boldsymbol{T}_{S \leftarrow D}\right]_{D}^{D} \overrightarrow{\boldsymbol{r}}^{C L} \\
& +\left[\boldsymbol{T}_{N \leftarrow S}\right]\left[\dot{\boldsymbol{T}}_{S \leftarrow D}\right]_{D}^{D} \overrightarrow{\boldsymbol{r}}^{C L}+\left[\boldsymbol{T}_{N \leftarrow S}\right]\left[\boldsymbol{T}_{S \leftarrow D}\right]_{D}^{D} \dot{\overrightarrow{\boldsymbol{r}}}^{C L} \\
& +\left[\dot{\boldsymbol{T}}_{N \leftarrow S}\right]\left[\boldsymbol{T}_{S \leftarrow D}\right]\left[\boldsymbol{T}_{D \leftarrow C L}\right]_{C L}^{C L} \overrightarrow{\boldsymbol{r}}^{C L_{m}}+\left[\boldsymbol{T}_{N \leftarrow S}\right]\left[\dot{\boldsymbol{T}}_{S \leftarrow D}\right]\left[\boldsymbol{T}_{D \leftarrow C L}\right]_{C L}^{C L} \overrightarrow{\boldsymbol{r}}^{C L_{m}} \\
& +\left[\boldsymbol{T}_{N \leftarrow S}\right]\left[\boldsymbol{T}_{S \leftarrow D}\right]\left[\dot{\boldsymbol{T}}_{D \leftarrow C L}\right]_{C L}^{C L} \overrightarrow{\boldsymbol{r}}^{C L_{m}}+\left[\boldsymbol{T}_{N \leftarrow S}\right]\left[\boldsymbol{T}_{S \leftarrow D}\right]\left[\boldsymbol{T}_{D \leftarrow C L}\right]_{C L}^{C L} \dot{\overrightarrow{\boldsymbol{r}}}^{C L_{m}}
\end{aligned}
$$

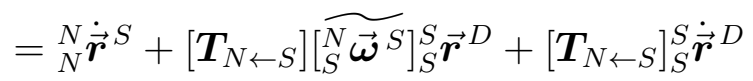

$$
\begin{aligned}
& +\left[\boldsymbol{T}_{N \leftarrow S}\right] \widetilde{\left.{ }_{S}^{N \overrightarrow{\boldsymbol{\omega}}^{S}}\right]}\left[\boldsymbol{T}_{S \leftarrow D}\right]_{D}^{D} \overrightarrow{\boldsymbol{r}}^{C L}+\left[\boldsymbol{T}_{N \leftarrow S}\right]\left[\boldsymbol{T}_{S \leftarrow D}\right]\left[\widetilde{{ }_{D}^{S \overrightarrow{\boldsymbol{\omega}}^{D}}}\right]_{D}^{D} \overrightarrow{\boldsymbol{r}}^{C L} \\
& +\left[\boldsymbol{T}_{N \leftarrow S}\right]\left[\boldsymbol{T}_{S \leftarrow D}\right]_{D}^{D} \dot{\overrightarrow{\boldsymbol{r}}} C L+\left[\boldsymbol{T}_{N \leftarrow S}\right]\left[\widetilde{N_{S}^{N} \overrightarrow{\boldsymbol{\omega}}^{S}}\right]\left[\boldsymbol{T}_{S \leftarrow D}\right]\left[\boldsymbol{T}_{D \leftarrow C L}\right]_{C L}^{C L} \overrightarrow{\boldsymbol{r}}^{C L_{m}} \\
& \left.+\left[\boldsymbol{T}_{N \leftarrow S}\right]\left[\boldsymbol{T}_{S \leftarrow D}\right] \widetilde{S_{D}^{S} \overrightarrow{\boldsymbol{\omega}}^{D}}\right]\left[\boldsymbol{T}_{D \leftarrow C L}\right]_{C L}^{C L} \overrightarrow{\boldsymbol{r}}^{C L_{m}} \\
& +\left[\boldsymbol{T}_{N \leftarrow S}\right]\left[\boldsymbol{T}_{S \leftarrow D}\right]\left[\boldsymbol{T}_{D \leftarrow C L}\right]\left[\widetilde{D_{C L}^{D} \overrightarrow{\boldsymbol{\omega}} C L}\right]_{C L}^{C L} \overrightarrow{\boldsymbol{r}}^{C L_{m}} \\
& +\left[\boldsymbol{T}_{N \leftarrow S}\right]\left[\boldsymbol{T}_{S \leftarrow D}\right]\left[\boldsymbol{T}_{D \leftarrow C L}\right]_{e L}^{C L} \dot{\overrightarrow{\boldsymbol{p}} C L_{m}} 0 \\
& \left.={ }_{N}^{N} \dot{\overrightarrow{\boldsymbol{r}}}^{S}+\left[\boldsymbol{T}_{N \leftarrow S}\right] \widetilde{{ }_{S}^{N} \overrightarrow{\boldsymbol{\omega}}^{S}}\right]_{S}^{S} \overrightarrow{\boldsymbol{r}}^{D}+\left[\boldsymbol{T}_{N \leftarrow S}\right]_{S}^{S} \dot{\overrightarrow{\boldsymbol{r}}}^{D}+\left[\boldsymbol{T}_{N \leftarrow S}\right]\left[\widetilde{N}_{S}^{N \overrightarrow{\boldsymbol{\omega}} S}\right]\left[\boldsymbol{T}_{S \leftarrow D}\right]_{D}^{D} \overrightarrow{\boldsymbol{r}}^{C L} \\
& +\left[\boldsymbol{T}_{N \leftarrow D}\right]\left[\widetilde{{ }_{D}^{S} \overrightarrow{\boldsymbol{\omega}} D}\right]_{D}^{D} \overrightarrow{\boldsymbol{r}}^{C L}+\left[\boldsymbol{T}_{N \leftarrow D}\right]_{D}^{D} \dot{\overrightarrow{\boldsymbol{r}}}^{C L}+\left[\boldsymbol{T}_{N \leftarrow S}\right] \widetilde{\left.{ }_{S}^{N \overrightarrow{\boldsymbol{\omega}}^{S}}\right]}\left[\boldsymbol{T}_{S \leftarrow C L}\right]_{C L}^{C L} \overrightarrow{\boldsymbol{r}}^{C L_{m}} \\
& \left.+\left[\boldsymbol{T}_{N \leftarrow D}\right]\left[\widetilde{{ }_{D}^{S} \overrightarrow{\boldsymbol{\omega}} D}\right]\left[\boldsymbol{T}_{D \leftarrow C L}\right]_{C L}^{C L} \overrightarrow{\boldsymbol{r}}^{C L_{m}}+\left[\boldsymbol{T}_{N \leftarrow C L}\right] \widetilde{\left[{ }_{C L} \overrightarrow{\boldsymbol{\omega}} C L\right.}\right]_{C L}^{C L} \overrightarrow{\boldsymbol{r}}^{C L_{m}}
\end{aligned}
$$


Similarly,

$$
\begin{aligned}
& { }_{N}^{N} \overrightarrow{\boldsymbol{v}}^{C R_{m}}=\frac{\mathrm{d}}{\mathrm{d} t}\left({ }_{N}^{N} \overrightarrow{\boldsymbol{r}}^{C R_{m}}\right) \\
& =\frac{\mathrm{d}}{\mathrm{d} t}\left({ }_{N}^{N} \overrightarrow{\boldsymbol{r}}^{S}\right)+\frac{\mathrm{d}}{\mathrm{d} t}\left(\left[\boldsymbol{T}_{N \leftarrow S}\right]_{S}^{S} \overrightarrow{\boldsymbol{r}}^{D}\right)+\frac{\mathrm{d}}{\mathrm{d} t}\left(\left[\boldsymbol{T}_{N \leftarrow S}\right]\left[\boldsymbol{T}_{S \leftarrow D}\right]_{D}^{D} \overrightarrow{\boldsymbol{r}}^{C R}\right) \\
& +\frac{\mathrm{d}}{\mathrm{d} t}\left(\left[\boldsymbol{T}_{N \leftarrow S}\right]\left[\boldsymbol{T}_{S \leftarrow D}\right]\left[\boldsymbol{T}_{D \leftarrow C R}\right]_{C R}^{C R} \overrightarrow{\boldsymbol{r}}^{C R_{m}}\right) \\
& ={ }_{N}^{N} \dot{\overrightarrow{\boldsymbol{r}}}^{S}+\left[\dot{\boldsymbol{T}}_{N \leftarrow S}\right]_{S}^{S} \overrightarrow{\boldsymbol{r}}^{D}+\left[\boldsymbol{T}_{N \leftarrow S}\right]_{S}^{S} \dot{\overrightarrow{\boldsymbol{r}}}^{D}+\left[\dot{\boldsymbol{T}}_{N \leftarrow S}\right]\left[\boldsymbol{T}_{S \leftarrow D}\right]_{D}^{D} \overrightarrow{\boldsymbol{r}}^{C R} \\
& +\left[\boldsymbol{T}_{N \leftarrow S}\right]\left[\dot{\boldsymbol{T}}_{S \leftarrow D}\right]_{D}^{D} \overrightarrow{\boldsymbol{r}}^{C R}+\left[\boldsymbol{T}_{N \leftarrow S}\right]\left[\boldsymbol{T}_{S \leftarrow D}\right]_{D}^{D} \dot{\overrightarrow{\boldsymbol{r}}}^{C R} \\
& +\left[\dot{\boldsymbol{T}}_{N \leftarrow S}\right]\left[\boldsymbol{T}_{S \leftarrow D}\right]\left[\boldsymbol{T}_{D \leftarrow C R}\right]_{C R}^{C R} \overrightarrow{\boldsymbol{r}}^{C R_{m}}+\left[\boldsymbol{T}_{N \leftarrow S}\right]\left[\dot{\boldsymbol{T}}_{S \leftarrow D}\right]\left[\boldsymbol{T}_{D \leftarrow C R}\right]_{C R}^{C R} \overrightarrow{\boldsymbol{r}}^{C R_{m}} \\
& +\left[\boldsymbol{T}_{N \leftarrow S}\right]\left[\boldsymbol{T}_{S \leftarrow D}\right]\left[\dot{\boldsymbol{T}}_{D \leftarrow C R}\right]_{C R}^{C R} \overrightarrow{\boldsymbol{r}}^{C R_{m}}+\left[\boldsymbol{T}_{N \leftarrow S}\right]\left[\boldsymbol{T}_{S \leftarrow D}\right]\left[\boldsymbol{T}_{D \leftarrow C R}\right]_{C R}^{C R} \dot{\overrightarrow{\boldsymbol{r}}} C R_{m} \\
& ={ }_{N}^{N} \dot{\overrightarrow{\boldsymbol{r}}}^{S}+\left[\boldsymbol{T}_{N \leftarrow S}\right]\left[\widetilde{{ }_{S}^{N} \overrightarrow{\boldsymbol{\omega}} S}\right]_{S}^{S} \overrightarrow{\boldsymbol{r}}^{D}+\left[\boldsymbol{T}_{N \leftarrow S}\right]_{S}^{S} \dot{\overrightarrow{\boldsymbol{r}}}^{D} \\
& \left.+\left[\boldsymbol{T}_{N \leftarrow S}\right] \widetilde{{ }_{S}^{N} \overrightarrow{\boldsymbol{\omega}}^{S}}\right]\left[\boldsymbol{T}_{S \leftarrow D}\right]_{D}^{D} \overrightarrow{\boldsymbol{r}}^{C R}+\left[\boldsymbol{T}_{N \leftarrow S}\right]\left[\boldsymbol{T}_{S \leftarrow D}\right]\left[\widetilde{S_{D} \overrightarrow{\boldsymbol{\omega}}^{D}}\right]_{D}^{D} \overrightarrow{\boldsymbol{r}}^{C R} \\
& +\left[\boldsymbol{T}_{N \leftarrow S}\right]\left[\boldsymbol{T}_{S \leftarrow D}\right]_{D}^{D} \dot{\overrightarrow{\boldsymbol{r}}} C R+\left[\boldsymbol{T}_{N \leftarrow S}\right]\left[\widetilde{N_{S}^{N} \overrightarrow{\boldsymbol{\omega}} S}\right]\left[\boldsymbol{T}_{S \leftarrow D}\right]\left[\boldsymbol{T}_{D \leftarrow C R}\right]_{C R}^{C R} \overrightarrow{\boldsymbol{r}}^{C R_{m}} \\
& +\left[\boldsymbol{T}_{N \leftarrow S}\right]\left[\boldsymbol{T}_{S \leftarrow D}\right]\left[\widetilde{S_{D}^{S} \overrightarrow{\boldsymbol{\omega}}^{D}}\right]\left[\boldsymbol{T}_{D \leftarrow C R}\right]_{C R}^{C R} \overrightarrow{\boldsymbol{r}}^{C R_{m}} \\
& +\left[\boldsymbol{T}_{N \leftarrow S}\right]\left[\boldsymbol{T}_{S \leftarrow D}\right]\left[\boldsymbol{T}_{D \leftarrow C R}\right]\left[\widetilde{D_{C R}^{[\overrightarrow{\boldsymbol{\omega}} C R}}\right]_{C R}^{C R} \overrightarrow{\boldsymbol{r}}^{C R_{m}} \\
& +\left[\boldsymbol{T}_{N \leftarrow S}\right]\left[\boldsymbol{T}_{S \leftarrow D}\right]\left[\boldsymbol{T}_{D \leftarrow C R}\right]_{e R}^{C R} \dot{\overrightarrow{\boldsymbol{p}}} C R_{m}-0 \\
& ={ }_{N}^{N} \dot{\overrightarrow{\boldsymbol{r}}}^{S}+\left[\boldsymbol{T}_{N \leftarrow S}\right]\left[\widetilde{{ }_{S}^{N} \overrightarrow{\boldsymbol{\omega}}^{S}}\right]_{S}^{S} \overrightarrow{\boldsymbol{r}}^{D}+\left[\boldsymbol{T}_{N \leftarrow S}\right]_{S}^{S} \dot{\overrightarrow{\boldsymbol{r}}}^{D}+\left[\boldsymbol{T}_{N \leftarrow S}\right]\left[\widetilde{{ }_{S}^{N} \overrightarrow{\boldsymbol{\omega}}^{S}}\right]\left[\boldsymbol{T}_{S \leftarrow D}\right]_{D}^{D} \overrightarrow{\boldsymbol{r}}^{C R} \\
& +\left[\boldsymbol{T}_{N \leftarrow D}\right]\left[\widetilde{S_{D}^{S} \overrightarrow{\boldsymbol{\omega}}^{D}}\right]_{D}^{D} \overrightarrow{\boldsymbol{r}}^{C R}+\left[\boldsymbol{T}_{N \leftarrow D}\right]_{D}^{D} \dot{\overrightarrow{\boldsymbol{r}}}^{C R}+\left[\boldsymbol{T}_{N \leftarrow S}\right]\left[\widetilde{{ }_{S}^{N} \overrightarrow{\boldsymbol{\omega}}^{S}}\right]\left[\boldsymbol{T}_{S \leftarrow C R}\right]_{C R}^{C R} \overrightarrow{\boldsymbol{r}}^{C R_{m}}
\end{aligned}
$$

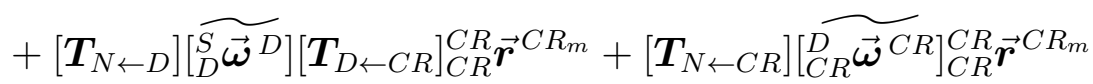

Equations 3.40 through 3.47 complete the definition of the polygons included in the SRAMSS environment. At this point the relevant kinematic expressions and quantities have been developed such that they are available for the application of Kane's method for deriving the governing equations of motion for the system. 


\section{Chapter 4}

\section{System Dynamics}

This chapter follows the development of the dynamics components required to complete the governing equations for the system using Kane's method, as outlined in Section 2.2. Ultimately, the objective of this chapter is to obtain the terms which form the active forces $\overrightarrow{\boldsymbol{R}}^{k}$ and moments $\overrightarrow{\boldsymbol{T}}^{k}$, the translational inertia forces $\overrightarrow{\boldsymbol{R}}^{* k}$ and angular inertia moments $\overrightarrow{\boldsymbol{T}}^{* k}$ for all bodies in the system.

As presented in Section 2.2, the governing equations of motion expressed in terms of $N_{g c}$ number of generalized coordinates in Kane's method as

$$
F_{i}+F_{i}^{*}=0, \quad i=1, \ldots, N_{g c}
$$

Vector formulation of Equation 2.11 for $N_{B}$ number of bodies, yields the following expressions for the active forces and inertial forces for the $k$ th body:

$$
\begin{aligned}
\boldsymbol{F} & =\sum_{k=1}^{N_{B}}\left(\left[{ }_{k}^{N} V^{k}\right]^{T} \overrightarrow{\boldsymbol{R}}^{k}+\left[{ }_{k}^{N} W^{k}\right]^{T} \overrightarrow{\boldsymbol{T}}^{k}\right) \\
\boldsymbol{F}^{*} & =\sum_{k=1}^{N_{B}}\left(\left[{ }_{k}^{N} V^{k}\right]^{T} \overrightarrow{\boldsymbol{R}}^{* k}+\left[{ }_{k}^{N} W^{k}\right]^{T} \overrightarrow{\boldsymbol{T}}^{* k}\right)
\end{aligned}
$$

For the given system, the aircraft is modelled as a single rigid body representing the airframe, coupled to a flexible dynamic finite element model representing the skidtype landing gear. Directly applying Kane's method would treat each DFE node as an independent body with properties associated with it based on conventional finite element formulation, and with its own governing equations. This method requires the development of the externally applied active forces, but also the internal forces acting between connected DFE nodes. For dense finite element meshes, this method proves to be analytically challenging. Alternatively, as will be shown in Section 4.1, the 
dynamic finite element model can be assembled separately using the 'direct method', then manipulated to fit the form of Equations 2.13 and 2.14 for direct integration into the governing equations.

\subsection{Dynamic Finite Element Model}

For reasons presented in Section 2.2, the skid-type landing gear is suited to dynamic finite element representation, and is therefore chosen as the method to model the compliant skid-type landing gear. Manipulation of the standard DFE formulation into a form usable in Kane's presents a novel method for the mass-coupling of rigid and flexible bodies in a system.

In general, a DFE model takes the form

$$
[\boldsymbol{M}] \ddot{\boldsymbol{q}}_{n}+[\boldsymbol{C}] \dot{\boldsymbol{q}}_{n}+[\boldsymbol{K}] \boldsymbol{q}_{n}=\boldsymbol{F}\left(\dot{\boldsymbol{q}}_{n}, \boldsymbol{q}_{n}\right)
$$

where $[\boldsymbol{M}]$ is the total structure consistent mass matrix, $[\boldsymbol{C}]$ is the total structure proportional damping matrix, $[\boldsymbol{K}]$ is the total structure stiffness matrix, and $\boldsymbol{F}\left(\dot{\boldsymbol{q}}_{n}, \boldsymbol{q}_{n}\right)$ is the vector of externally applied nodal forces acting on the DFE model. The construction of the stiffness, mass, and damping matrices is presented below.

\section{Stiffness Matrix}

The stiffness matrix is constructed using the direct method [51]. That is, to apply known principles to individual elements then assemble them into the matrices governing the complete finite element model. Limited to simple elements, the stiffness matrix is obtained through known mechanics of materials formulas relating node displacements and node loads.

The general formula for the local element stiffness matrix $\left[\boldsymbol{K}_{l}\right]$ is derived from the expression for strain energy per unit of volume $U_{0}$ in a flexible material integrated over the total volume of the element [51]:

$$
\begin{aligned}
U & =\frac{1}{2} \boldsymbol{\delta}^{T} \int \boldsymbol{\beta}^{T} \boldsymbol{\epsilon} \boldsymbol{\beta} \mathrm{d} V \boldsymbol{\delta} \\
& =\frac{1}{2} \boldsymbol{\delta}^{T} \boldsymbol{k} \boldsymbol{\delta}
\end{aligned}
$$

with

$$
\boldsymbol{k}=\int \boldsymbol{\beta}^{T} \boldsymbol{\epsilon} \boldsymbol{\beta} \mathrm{d} V
$$


where $\boldsymbol{\delta}$ is a vector of the nodal displacements along each DoF, $\boldsymbol{\epsilon}$ the constitutive, or material property matrix, and $\boldsymbol{\beta}$ the strain-displacement matrix resulting from the partial differentiation of polynomial interpolation shape functions $\boldsymbol{N}$ with respect to the nodal DoFs.

The landing gear is modelled using general 2D beam elements which resists, axial, shear, and bending loads. As shown in Figure 4.1, the general 2D beam elements have 6 DoFs. The element is composed of the superposition of a bar element which resists axial loads, and a simple 2D beam element which resists shear and bending loads.

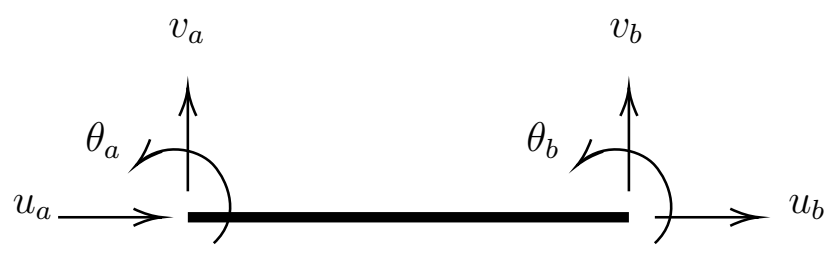

Figure 4.1: 2D beam element degrees of freedom.

For the general 2D beam element, $\boldsymbol{\delta}$ are

$$
\boldsymbol{\delta}=\left\{\begin{array}{l}
u_{a} \\
v_{a} \\
\theta_{a} \\
u_{b} \\
v_{b} \\
\theta_{b}
\end{array}\right\}
$$

Due to superposition of element types, the shape functions $\boldsymbol{N}$ for the general $2 \mathrm{D}$ beam element, which agrees with the DoFs in $\boldsymbol{\delta}$, is

$$
\boldsymbol{N}=\left[\begin{array}{cccccc}
\frac{L-x}{L} & 0 & 0 & \frac{x}{L} & 0 & 0 \\
0 & 1-\frac{3 x^{2}}{L^{2}}+\frac{2 x^{3}}{L^{3}} & x-\frac{2 x^{2}}{L}+\frac{x^{3}}{L^{2}} & 0 & \frac{3 x^{2}}{L^{2}}-\frac{2 x^{3}}{L^{3}} & -\frac{x^{2}}{L}+\frac{x^{3}}{L^{2}}
\end{array}\right]
$$

where the top row is the bar-element contribution, and the bottom row is the simple $2 \mathrm{D}$ beam element contribution. Differentiation $\frac{\mathrm{d}}{\mathrm{d}^{2} x}$ yields the strain-deflection matrix $\boldsymbol{\beta}:$

$$
\boldsymbol{\beta}=\frac{\mathrm{d}^{2}}{\mathrm{~d} x^{2}} \boldsymbol{N}=\left[\begin{array}{cccccc}
\frac{-1}{L} & 0 & 0 & \frac{1}{L} & 0 & 0 \\
0 & -\frac{6}{L^{2}}+\frac{12 x}{L^{3}} & -\frac{4}{L}+\frac{6}{L^{2}} & 0 & \frac{6}{L^{2}}-\frac{12 x}{L^{3}} & -\frac{2}{L}+\frac{6}{L^{2}}
\end{array}\right]
$$


and, again with element superposition, the material property matrix $\boldsymbol{\epsilon}$ :

$$
\boldsymbol{\epsilon}=\left[\begin{array}{cc}
A E & 0 \\
0 & E I
\end{array}\right]
$$

where $L, A, E$, and $I$ are the element length, cross-sectional area, Young's modulus, and second moment of area respectively.

With these matrices, computing Equation 4.2 yields the general 2D beam element stiffness matrix in the local frame of reference:

$$
\left[\boldsymbol{K}_{l}\right]=\int_{0}^{L} \boldsymbol{\beta}^{T} \boldsymbol{\epsilon} \boldsymbol{\beta} \mathrm{d} x=\left[\begin{array}{cccccc}
\frac{A E}{L} & 0 & 0 & -\frac{A E}{L} & 0 & 0 \\
0 & \frac{12 E I}{L^{3}} & \frac{6 E I}{L^{2}} & 0 & -\frac{12 E I}{L^{3}} & \frac{6 E I}{L^{2}} \\
0 & \frac{6 E I}{L^{2}} & \frac{4 E I}{L} & 0 & -\frac{6 E I}{L^{2}} & \frac{2 E I}{L} \\
-\frac{A E}{L} & 0 & 0 & \frac{A E}{L} & 0 & 0 \\
0 & -\frac{12 E I}{L^{3}} & -\frac{6 E I}{L^{2}} & 0 & \frac{12 E I}{L^{3}} & -\frac{6 E I}{L^{2}} \\
0 & \frac{6 E I}{L^{2}} & \frac{2 E I}{L} & 0 & -\frac{6 E I}{L^{2}} & \frac{4 E I}{L}
\end{array}\right]
$$

Equation 4.7 presents the stiffness matrix for a single general 2D beam element, yet the landing gear DFE model is composed of many elements. Since a DFE node may connect more than one element, the frames of reference of connecting beams must be consistently axis-aligned to determine the deflection at the nodes connecting multiple elements. In this work, these aligned frames of reference for each DFE node are the $j$ th DFE node frames $n_{j}$, as presented in Section 2.3. These frames of reference are all consistently axis-aligned with the global frame $G$. The $G$ frame serves as the global finite element model frame of reference, and isolates the description of the DFE model from the airframe's coordinate system $H$ which could potentially see transient effects in future work.

Obtaining the total structure stiffness matrix $[\boldsymbol{K}]$ requires the assembly of all defined elements. This is achieved by transforming the arbitrarily-oriented element at angle $\Theta_{a b}$ to the global frame, spanning from node $a$ to node $b$, from local frames of references $n_{a}^{\prime}$ and $n_{b}^{\prime}$ to globally aligned frames $n_{a}$ and $n_{b}$ as shown in Figure 4.2.

The transformation matrix describing the transformation global DoFs to local 


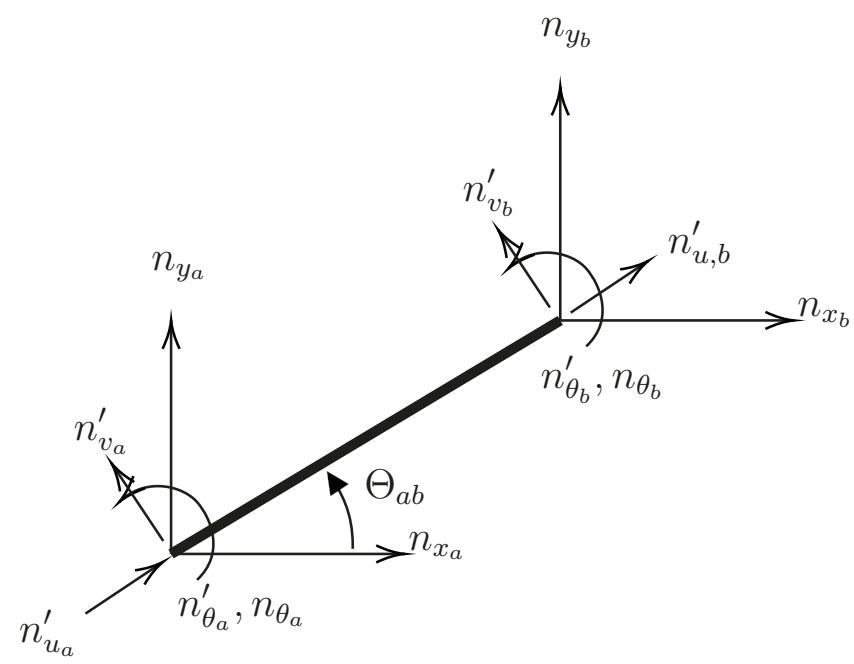

Figure 4.2: Arbitrarily-oriented 2D beam element frames of reference.

DoFs is

$$
\begin{aligned}
{\left[\boldsymbol{T}_{n_{a b}^{\prime} \leftarrow n_{a b}}\right]=} & {\left[\begin{array}{cc}
{\left[\boldsymbol{T}_{a^{\prime} \leftarrow a}\right]} & {[0]} \\
{[0]} & {\left[\boldsymbol{T}_{b^{\prime} \leftarrow b}\right]}
\end{array}\right] } \\
= & {\left[\begin{array}{cccccc}
\cos \left(\Theta_{a b}\right) & -\sin \left(\Theta_{a b}\right) & 0 & 0 & 0 & 0 \\
\sin \left(\Theta_{a b}\right) & \cos \left(\Theta_{a b}\right) & 0 & 0 & 0 & 0 \\
0 & 0 & 1 & 0 & 0 & 0 \\
0 & 0 & 0 & \cos \left(\Theta_{a b}\right) & -\sin \left(\Theta_{a b}\right) & 0 \\
0 & 0 & 0 & \sin \left(\Theta_{a b}\right) & \cos \left(\Theta_{a b}\right) & 0 \\
0 & 0 & 0 & 0 & 0 & 1
\end{array}\right] }
\end{aligned}
$$

Therefore the relationship between globally-aligned and local DoFs is

$$
\begin{aligned}
\boldsymbol{\delta}_{l} & =\left[\boldsymbol{T}_{n_{a b}^{\prime} \leftarrow n_{a b}}\right] \boldsymbol{\delta}_{G} \\
\boldsymbol{\delta}_{G} & =\left[\boldsymbol{T}_{n_{a b}^{\prime} \leftarrow n_{a b}}\right]^{T} \boldsymbol{\delta}_{l}
\end{aligned}
$$

The transformation matrix can be used to determine the globally aligned stiffness matrix. The transpose of the transformation matrix is used since it is a homogenous rotational transformation matrix where $[\boldsymbol{A}]^{-1}=[\boldsymbol{A}]^{T}$. In transforming the local forces and deflection to global quantities, we have

$$
\begin{aligned}
{\left[\boldsymbol{K}_{l}\right] \boldsymbol{\delta}_{l} } & =\boldsymbol{F}_{l} \\
{\left[\boldsymbol{K}_{l}\right]\left[\boldsymbol{T}_{n_{a b}^{\prime} \leftarrow n_{a b}}\right] \boldsymbol{\delta}_{G} } & =\left[\boldsymbol{T}_{n_{a b}^{\prime} \leftarrow n_{a b}}\right] \boldsymbol{F}_{G} \\
{\left[\boldsymbol{T}_{n_{a b}^{\prime} \leftarrow n_{a b}}\right]^{T}\left[\boldsymbol{K}_{l}\right]\left[\boldsymbol{T}_{n_{a b}^{\prime} \leftarrow n_{a b}}\right] \boldsymbol{\delta}_{G} } & =\left[\boldsymbol{T}_{n_{a b}^{\prime} \leftarrow n_{a b}}\right]^{T}\left[\boldsymbol{T}_{n_{a b}^{\prime} \leftarrow n_{a b}}\right] \boldsymbol{F}_{G} \\
{\left[\boldsymbol{T}_{n_{a b}^{\prime} \leftarrow n_{a b}}\right]^{T}\left[\boldsymbol{K}_{l}\right]\left[\boldsymbol{T}_{n_{a b}^{\prime} \leftarrow n_{a b}}\right] \boldsymbol{\delta}_{G} } & =\boldsymbol{F}_{G}
\end{aligned}
$$


By inspection, the general 2D beam element stiffness matrix expressed in the globallyaligned frame is obtained in Equation 4.12.

$$
\left[\boldsymbol{K}_{G, a b}\right]=\left[\boldsymbol{T}_{n_{a b}^{\prime} \leftarrow n_{a b}}\right]^{T}\left[\boldsymbol{K}_{l, a b}\right]\left[\boldsymbol{T}_{n_{a b}^{\prime} \leftarrow n_{a b}}\right]
$$

Further, the element stiffness matrix spanning nodes $a$ and $b$, expressed in the global frame, is partitioned to obtain sub-matrices which isolate the influence of both nodes on the displacement in each DoF.

$$
\begin{array}{r}
{\left[\boldsymbol{K}_{G}\right]\left\{\begin{array}{l}
\boldsymbol{\delta}_{a} \\
\boldsymbol{\delta}_{b}
\end{array}\right\}=\left\{\begin{array}{l}
\boldsymbol{F}_{a} \\
\boldsymbol{F}_{b}
\end{array}\right\}} \\
{\left[\begin{array}{ll}
\boldsymbol{k}_{G, a a} & \boldsymbol{k}_{G, a b} \\
\boldsymbol{k}_{G, b a} & \boldsymbol{k}_{G, b b}
\end{array}\right]\left\{\begin{array}{l}
\boldsymbol{\delta}_{a} \\
\boldsymbol{\delta}_{b}
\end{array}\right\}=\left\{\begin{array}{l}
\boldsymbol{F}_{a} \\
\boldsymbol{F}_{b}
\end{array}\right\}}
\end{array}
$$

Equation 4.12 is applied to each element of the DFE model, then the stiffness matrix is partitioned as per Equation 4.13. These partitions are assembled directly to obtain the total structure stiffness matrix $[\boldsymbol{K}]$, which is known as the 'direct method'.

$$
[\boldsymbol{K}]=\left[\begin{array}{cccc}
\boldsymbol{k}_{11} & \boldsymbol{k}_{1 j_{c}} & \cdots & \boldsymbol{k}_{1 N_{n}} \\
\boldsymbol{k}_{j_{r} 1} & \boldsymbol{k}_{j_{r} j_{c}} & & \vdots \\
\vdots & & \ddots & \vdots \\
\boldsymbol{k}_{N_{n} 1} & \cdots & \cdots & \boldsymbol{k}_{N_{n} N_{n}}
\end{array}\right]
$$

where each element $\boldsymbol{k}_{j_{r} j_{c}}$, with row index $r$ and column index $c$, of the total structure stiffness matrix $[\boldsymbol{K}]$ is the summation of all element globally-aligned stiffness matrix partitions contributing to the stiffness of the given node.

$$
\boldsymbol{k}_{j_{r} j_{c}}=\sum_{a=j_{r}, b=j_{c}} \boldsymbol{k}_{G, a b}, \quad j_{r}, j_{c}=1, \ldots, N_{n}
$$

The associated nodal displacement vector is $\boldsymbol{q}_{n}$ from Equation 2.21). For the system at hand, it is defined as $\boldsymbol{\delta}_{n}$. Since the forces generated through deflection are due to nodal displacements, it suffices to define the nodal displacements to be the difference of generalized coordinates of the nodes with respect to the global frame, and the undeformed coordinates of the nodes with respect to the global frame, such that

$$
\boldsymbol{\delta}_{n}=\left\{\begin{array}{c}
\boldsymbol{\delta}_{n_{1}} \\
\vdots \\
\boldsymbol{\delta}_{n_{j}}
\end{array}\right\}=\left\{\begin{array}{c}
\boldsymbol{q}_{n_{1}} \\
\vdots \\
\boldsymbol{q}_{n_{j}}
\end{array}\right\}-\left\{\begin{array}{c}
\boldsymbol{q}_{n_{1}, 0} \\
\vdots \\
\boldsymbol{q}_{n_{j}, 0}
\end{array}\right\}=\left\{\begin{array}{c}
{ }_{G}^{G} \overrightarrow{\boldsymbol{r}}^{n_{1}} \\
{ }_{G}^{G} \overrightarrow{\boldsymbol{\theta}}^{n_{1}} \\
\vdots \\
{ }_{G}^{G} \overrightarrow{\boldsymbol{r}}^{n_{j}} \\
{ }_{G}^{G} \overrightarrow{\boldsymbol{\theta}}^{n_{j}}
\end{array}\right\}-\left\{\begin{array}{c}
{ }_{G}^{G} \overrightarrow{\boldsymbol{r}}^{n_{1}}{ }_{0} \\
{ }_{G}^{G} \overrightarrow{\boldsymbol{\theta}}^{n_{1}}{ }_{0} \\
\vdots \\
{ }_{G}^{G} \overrightarrow{\boldsymbol{r}}^{n_{j}}{ }_{0}{ }_{G} \overrightarrow{\boldsymbol{\theta}}^{n_{j}}{ }_{0}
\end{array}\right\}, j=1, \ldots, N_{n}
$$


where ${ }_{G}^{G} \overrightarrow{\boldsymbol{r}}^{n_{j}}$ is the undeformed position of the $j$ th DFE node relative to the global DFE frame $G$, expressed in $G$; and $G_{G}^{G} \overrightarrow{\boldsymbol{\theta}}^{n_{j}}$ is the undeformed orientation of the $j$ th DFE node with respect to $G$, expressed in $G$. In the undeformed configuration, nodal angular displacements are all zero such that

$$
{ }_{G}^{G} \overrightarrow{\boldsymbol{\theta}}^{n_{j}} \equiv 0
$$

Even though the governing equations are expressed in the $k$ th body frame, since the transformations from the node body frame $n_{j}$ to global DFE frame $G$ is identity, generalized coordinates may be used directly. For simulation purposes, it is preferable to use a separate vector for the undeformed DFE node generalized coordinates rather than the vector of initial conditions for the generalized coordinates of the DFE nodes to allow for deformed landing gear initial conditions.

\section{Mass Matrix}

The element mass matrix $\left[\boldsymbol{M}_{l}\right]$ in the local frame is derived in a similar manner to the development of the element stiffness matrix $\left[\boldsymbol{K}_{l}\right]$. Shape functions $\boldsymbol{N}$ are used in both cases. For the element mass matrix, Cook [51] uses inertial forces in virtual work arguments to obtain a general formula similar to Equation 4.2.

$$
\begin{aligned}
\boldsymbol{m} & =\rho \int \boldsymbol{N}^{T} \boldsymbol{N} d V \\
& =\rho A \int \boldsymbol{N}^{T} \boldsymbol{N} d x
\end{aligned}
$$

where $\rho$ and $A$ are the element density and cross-sectional area perpendicular to the longitudinal axis respectively. Equation 4.18 generates the 'consistent' mass matrix as it uses the same shape functions as the element stiffness matrix. Equation 4.18 yields

$$
\left[\boldsymbol{M}_{l}\right]=\frac{\rho A L}{420}\left[\begin{array}{cccccc}
140 & 0 & 0 & 70 & 0 & 0 \\
0 & 156 & 22 L & 0 & 54 & -13 L \\
0 & 22 L & 4 L^{2} & 0 & -13 L & -3 L^{2} \\
70 & 0 & 0 & 140 & 0 & 0 \\
0 & 54 & 13 L & 0 & 156 & -22 L \\
0 & -13 L & -3 L^{2} & 0 & -22 L & 4 L^{2}
\end{array}\right]
$$

Using the same method as Equation 4.11 and equating $[\boldsymbol{M}] \ddot{\boldsymbol{q}}=\boldsymbol{F}$, the globallyaligned element mass matrix takes the form

$$
\left[\boldsymbol{M}_{G}\right]=\left[\boldsymbol{T}_{n_{a b}^{\prime} \leftarrow n_{a b}}\right]^{T}\left[\boldsymbol{M}_{l}\right]\left[\boldsymbol{T}_{n_{a b}^{\prime} \leftarrow n_{a b}}\right]
$$


The globally-aligned element mass matrices are assembled into the total structure consistent mass matrix $[\boldsymbol{M}]$ in the same way as the stiffness matrix.

The associated nodal accelerations vector is $\ddot{\boldsymbol{q}}_{n}$ per Equation 2.21. Since the governing equations for the DFE nodes are expressed in their respective body frames, but accelerations require definition with respect to the inertial frame, the associated accelerations vector is assembled from the superposition of Equation 3.11 for all DFE nodes. Then the nodal accelerations vector becomes

$$
\ddot{\boldsymbol{q}}_{n}=\left(\left[\boldsymbol{V}_{n}\right] \dot{\boldsymbol{u}}+\boldsymbol{Z}_{n}\right)
$$

where $\left[\boldsymbol{V}_{n}\right]$ is the array of all translational and angular partial velocity matrices for all nodes in the DFE model, assembled as:

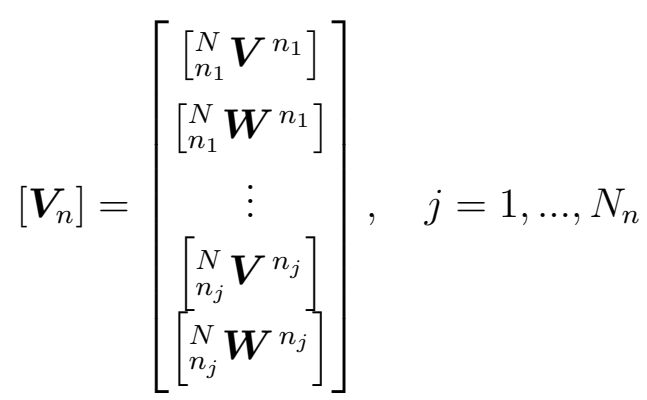

and $\boldsymbol{Z}_{n}$ is the column array of all remainder acceleration terms in the governing translational and angular acceleration equations for all DFE nodes in the form

$$
\boldsymbol{Z}_{n}=\left\{\begin{array}{c}
\overrightarrow{\boldsymbol{Z}}_{n_{1}} \\
\overrightarrow{\boldsymbol{Y}}_{n_{1}} \\
\vdots \\
\overrightarrow{\boldsymbol{Z}}_{n_{j}} \\
\overrightarrow{\boldsymbol{Y}}_{n_{j}}
\end{array}\right\}, \quad j=1, \ldots, N_{n}
$$

Expression of the mass matrix as a consistent mass matrix, and related nodal accelerations in the form of Equation 4.21 is advantageous for two reasons. First, the consistent mass matrix is able to capture nodal inertial phenomena in both translational and angular degrees of freedom. Second, expression of the accelerations using the partial velocity compact formulation allows direct assembly into Kane's governing equations, and subsequently the isolation of the generalized accelerations vector $\dot{\boldsymbol{u}}$.

\section{Proportional Damping Matrix}

Damping in structures arising from internal phenomena is difficult to mathematically characterize. However, damping forces in rigid structures often comprise less than 
$10 \%$ of forces in the system, and thus can be ideally modelled as viscous damping [51]. Mode superposition, assuming proportional damping, can be used to construct the total damping in a structure since element damping matrices cannot be easily formulated [50]. For this work, an explicitly-evaluated damping matrix is required to avoid recalculation of the total structure damping matrix $[\boldsymbol{C}]$ for each system solution evaluation.

The proportional damping matrix, as its name implies, is proportional to the mass matrix $[\boldsymbol{M}]$ and stiffness matrix $[\boldsymbol{K}]$. As presented in Equation 2.23 the total structure proportional damping matrix is

$$
[\boldsymbol{C}]=\alpha_{0}[\boldsymbol{M}]+\alpha_{1}[\boldsymbol{K}]
$$

where Equation 2.23, is the expansion of Equation 2.22 for the first two terms. Using the first two terms to generate proportional damping is often referred to as 'Rayleigh damping'. The proportionality constants $\alpha$ are obtained by solving the simultaneous equations in Equation 2.24 for the range of frequencies of interest $\omega_{1}$ to $\omega_{2}$ with associated damping ratios $\xi_{1}$ and $\xi_{2}[51]$ :

$$
\xi_{1}=\frac{\alpha_{0}}{2 \omega_{1}}+\frac{\alpha_{1} \omega_{1}}{2} \quad \xi_{2}=\frac{\alpha_{0}}{2 \omega_{2}}+\frac{\alpha_{1} \omega_{2}}{2}
$$

The nodal velocities $\dot{\boldsymbol{q}}_{n}$ are constructed from the generalized speeds associated with the DFE nodes such that

$$
\dot{\boldsymbol{q}}_{n}=\left[\boldsymbol{V}_{n}\right] \boldsymbol{u}
$$

\section{Complete Dynamic Finite Element Model}

Assembling the finite element matrices and associated nodal vectors yields a system of the form:

$$
[\boldsymbol{M}]\left(\left[\boldsymbol{V}_{n}\right] \dot{\boldsymbol{u}}+\boldsymbol{Z}_{n}\right)+[\boldsymbol{C}]\left[\boldsymbol{V}_{n}\right] \boldsymbol{u}+[\boldsymbol{K}] \boldsymbol{\delta}_{n}={ }_{n}^{n} \boldsymbol{F}
$$

where $\dot{\boldsymbol{u}}$ is the vector of generalized accelerations; the local time derivatives of the vector of generalized speeds $\boldsymbol{u}$, and ${ }_{n}^{n} \boldsymbol{F}$ is the vector of externally-applied nodal forces.

For the generic skid-equipped aircraft shown in Figure 2.2, the DFE-modelled skids are attached to the rigid airframe of the aircraft. The interface forces must be considered in the formulation of Kane's equations for both the aircraft's airframe and the flexible DFE landing gear model. In Equation 4.25, the external nodal forces ${ }_{n}^{n} \boldsymbol{F}$ can be separated into the known externally applied nodal forces ${ }_{n}^{n} \boldsymbol{F}_{k}$, and the unknown 
interface nodal forces ${ }_{n}^{n} \boldsymbol{F}_{u}$ which act at the nodes interfacing with the aircraft's rigid body.

$$
[\boldsymbol{M}]\left(\left[\boldsymbol{V}_{n}\right] \dot{\boldsymbol{u}}+\boldsymbol{Z}_{n}\right)+[\boldsymbol{C}]\left[\boldsymbol{V}_{n}\right] \boldsymbol{u}+[\boldsymbol{K}] \boldsymbol{\delta}_{n}={ }_{n}^{n} \boldsymbol{F}_{k}+{ }_{n}^{n} \boldsymbol{F}_{u}
$$

For the DFE nodes, Equation 4.26 can be manipulated into the form of Equations 2.13 and 2.14 as required for Kane's method, resulting in

$$
\begin{aligned}
\boldsymbol{F} & \left.=\left[\boldsymbol{V}_{n}\right]^{T}{ }_{n}^{n} \boldsymbol{F}_{k}+{ }_{n}^{n} \boldsymbol{F}_{u}-[\boldsymbol{C}]\left[\boldsymbol{V}_{n}\right] \boldsymbol{u}-[\boldsymbol{K}] \boldsymbol{\delta}_{n}\right) \\
& =\left[\boldsymbol{V}_{n}\right]^{T}\left({ }_{n}^{n} \boldsymbol{F}_{k}-[\boldsymbol{C}]\left[\boldsymbol{V}_{n}\right] \boldsymbol{u}-[\boldsymbol{K}] \boldsymbol{\delta}_{n}\right)+\left[\boldsymbol{V}_{u}\right]^{T}{ }_{n}^{n} \boldsymbol{F}_{u} \\
\boldsymbol{F}^{*} & =-\left[\boldsymbol{V}_{n}\right]^{T}\left([\boldsymbol{M}]\left(\left[\boldsymbol{V}_{n}\right] \dot{\boldsymbol{u}}+\boldsymbol{Z}_{n}\right)\right)
\end{aligned}
$$

In effect, the bracketed terms in the first line of Equation 4.27 are the active translational and angular forces $\boldsymbol{R}^{n}+\boldsymbol{T}^{n}$ acting on all DFE nodes. The bracketed terms in Equation 4.28 constitute the translational and angular inertia forces $\boldsymbol{R}^{* n}+\boldsymbol{T}^{* n}$ associated with the DFE nodes.

The known external nodal forces vector ${ }_{n}^{n} \boldsymbol{F}_{k}$ is the sum of all known external nodal forces, expressed

$$
{ }_{n}^{n} \boldsymbol{F}_{k}=\left\{\begin{array}{c}
{ }_{n_{1}} \overrightarrow{\boldsymbol{F}}_{k} \\
{ }_{n_{1}} \overrightarrow{\boldsymbol{M}}_{k} \\
\vdots \\
{ }_{1} \\
{ }_{n_{j}} \overrightarrow{\boldsymbol{F}}_{k} \\
{ }_{n_{j}} \overrightarrow{\boldsymbol{M}}_{k} \\
n_{j}
\end{array}\right\}=\left\{\begin{array}{c}
{ }_{n_{1}} \overrightarrow{\boldsymbol{F}}^{c}{ }_{k}+{ }_{{ }_{1}}^{n_{1}} \overrightarrow{\boldsymbol{F}}^{d}{ }_{k}+{ }_{n_{1}}^{n_{1}} \overrightarrow{\boldsymbol{F}}^{g}{ }_{k} \\
{ }_{n_{1}} \overrightarrow{\boldsymbol{M}}^{c}{ }_{k}+{ }_{n_{1}}^{n_{1}} \overrightarrow{\boldsymbol{M}}^{d}{ }_{k}+{ }_{n_{1}}^{n_{1}} \overrightarrow{\boldsymbol{M}}^{g}{ }_{k} \\
\vdots \\
n_{1} \\
{ }_{n_{j}} \overrightarrow{\boldsymbol{F}}^{c}{ }_{k}+{ }_{n_{j}}^{n_{j}} \overrightarrow{\boldsymbol{F}}^{d}{ }_{k}+{ }_{n_{j}}^{n_{j}} \overrightarrow{\boldsymbol{F}}^{g}{ }_{k} \\
{ }_{n_{j}} \overrightarrow{\boldsymbol{M}}^{c}{ }_{k}+{ }_{n_{j}}^{n_{j}} \overrightarrow{\boldsymbol{M}}^{d}{ }_{k}+{ }_{n_{j}} \overrightarrow{\boldsymbol{M}}^{g}{ }_{k}
\end{array}\right\}, \quad j=1, \ldots, N_{n}
$$

where the known nodal external forces are denoted with superscripts $c$ for contact forces, $d$ for external damper forces, and $g$ for gravitational forces. These known forces are obtained through individual models developed in the following sections of Chapter 4 .

The unknown interface nodal forces vector ${ }_{n}^{n} \boldsymbol{F}_{u}$ is expressed as a separate active forces term since it represents the unknown internal forces at the interface of the airframe and landing gear which must be solved. The set of DFE nodes with indices $j=j_{u}$ are nodes which interface with the airframe, such that the unknown force vector is

$$
{ }_{n}^{n} \boldsymbol{F}_{u}=\left\{\begin{array}{c}
c_{1}^{n_{1}} \overrightarrow{\boldsymbol{F}}_{n_{1}} \\
n_{1} \overrightarrow{\boldsymbol{M}}_{u} \\
\vdots \\
n_{1} \\
n_{j} \overrightarrow{\boldsymbol{F}}_{u} \\
n_{j} \overrightarrow{\boldsymbol{M}}_{u} \\
n_{j}
\end{array}\right\}, \quad j=1, \ldots, N_{n} \forall j=j_{u}
$$


with associated nodal partial velocities array

$$
\left[\boldsymbol{V}_{u}\right]=\left[\begin{array}{c}
{\left[\begin{array}{c}
N \\
n_{1}
\end{array} \boldsymbol{V}^{n_{1}}\right]} \\
{\left[\begin{array}{c}
N \\
n_{1}
\end{array} \boldsymbol{W}^{n_{1}}\right]} \\
\vdots \\
{\left[\begin{array}{c}
N \\
n_{j}
\end{array} \boldsymbol{V}^{n_{j}}\right]} \\
{\left[\begin{array}{l}
N \\
n_{j}
\end{array} \boldsymbol{W}^{n_{j}}\right]}
\end{array}\right], \quad j=1, \ldots, N_{n} \forall j=j_{u}
$$

constructed in the same way as Equation 4.22. The unknown interface forces are solved along with the unknown generalized accelerations, as will be shown in Chapter 5 .

wo In summary, manipulation of the DFE formulation into the form of Equations 4.27 and 4.28 , the forcing of $\left[\boldsymbol{T}_{G \leftarrow n_{j}}\right]=\equiv[I]$, and the separation of the nodal forces into ${ }_{n}^{n} \boldsymbol{F}_{k}$, and ${ }_{n}^{n} \boldsymbol{F}_{u}$ comprise the novel way of mass-coupling rigid and flexible bodies in Kane's method. The final consideration for integration of DFE modelling into Kane's method is presented in Section 4.4.1

\subsection{Contact Dynamics Model}

Transient intermittent contact states will arise due to the stiff nature of the skidtype landing gear. To accurately model this phenomena, a contact model which can characterize the complex contact states must be implemented. The contact dynamics must be able to detect colliding objects, and render an appropriately-oriented dynamic response for contact restitution. The implemented model leverages unit vectors normal and parallel to the polygon surfaces, generated for the Separation axis theorem collision detection algorithm, to apply normal and friction forces atthe contact surface. The contact dynamics model implemented in SRAMSS constitutes a novel and effective application of the Separation Axis Theorem [33] beyond simple collision detection into a general contact dynamics model.

\section{Contact Detection}

As it relates to the aircraft, the DFE model nodes act as the contact points evaluated for contact response. Contact force vectors ${ }_{n_{j}}^{n_{j}} \overrightarrow{\boldsymbol{F}}_{k}{ }_{k}$ are generated for each contacting node $n_{j_{c}}$, which are then assembled into the known external nodal forces vector ${ }_{n}^{n} \boldsymbol{F}_{k}$ 
in Equation 4.29. The set of DFE nodes with indices $j=j_{c}$ are nodes evaluated for contact response.

The objects with which the aircraft interacts, are often simple polygons with flat surfaces and well-defined geometries. As previously described in Section 2.3, the number of polygons is limited to the four polygons describing the ship deck, RSD deck, and two wedge clamps. based on the collision detection review presented in 1.3, these characteristics lend themselves to a narrow-phase, BVH contact detection algorithms [31, 32]. The limited number of polygons is small enough to skip broadphase detection and proceed directly to evaluating all polygons for contact in the narrow-phase. Since the defined polygons are physical representations, BVH is better suited than SPR as the Bounding volumes can exactly take the form of the defined polygons. The SAT is effective in this regard, and is implemented in the SRAMSS environment.

Simply, the SAT aims to determine separation of polygons evaluated for contact along an arbitrary axis as shown in Figure 4.3; inability to find such an axis implies contact.

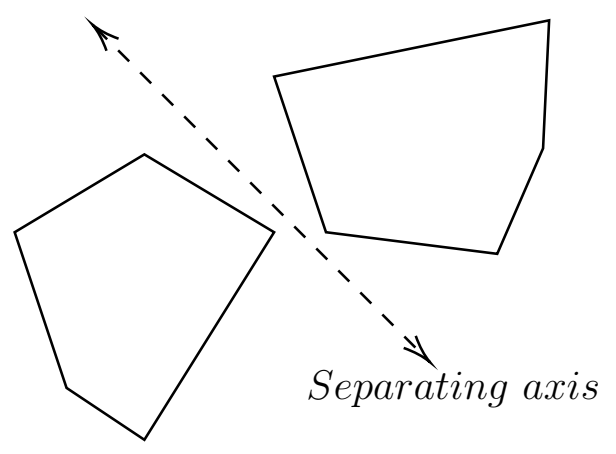

Figure 4.3: Arbitrary axis separating two polygons.

To evaluate two polygons for separation, the SAT requires outward-pointing normal axes ${ }_{N}^{P} \hat{\boldsymbol{s}}_{m}$ for each surface $s_{m}$ of polygon $P$. As shown in Figure 4.4, separation at surface ${ }^{P} s_{1}$ of polygon $P$ is determined by projecting all vertices of evaluated polygons $P$ and $Q$ to surface normal ${ }_{N}^{P} \hat{\boldsymbol{s}}_{1}$. Separation occurs where there is no overlap in the projection of the vertices from both polygons. This process is repeated for each surface of polygon $P$. If no separation can be found along any surface normal, the two polygons are determined to be in contact.

For this work, to simplify the SAT algorithm and increase computational efficiency, the DFE nodes are treated as points, and only the DFE nodes $n_{j}$ with indices $j$ 


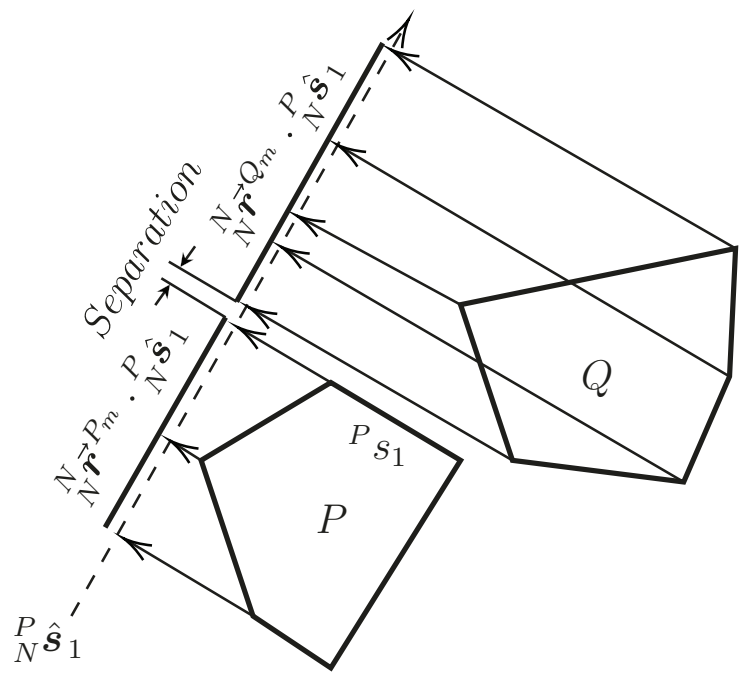

Figure 4.4: Separation of polygons $P$ and $Q$ along surface normal axis ${ }_{N}^{P} \hat{\boldsymbol{s}}_{1}$.

belonging to the set $j_{c}$ are evaluated for contact. The considerations reduce the number of pairs evaluated for contact response. Additionally, only the forces acting on contact nodes are required since the polygon motions are prescribed. It also reduces the number of point projection operations since only a single point is required to be projected to the normal axis for the DFE node evaluated for contact.

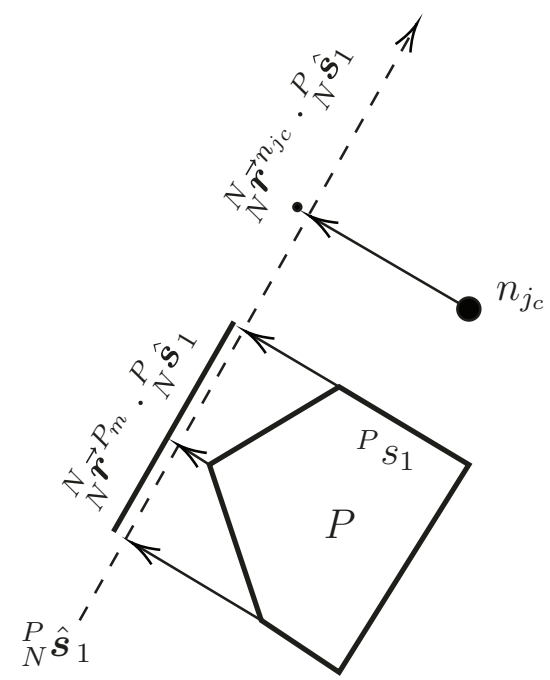

Figure 4.5: Separation between DFE node $n_{j_{c}}$ and defined polygon $P$.

The outward-facing normal axes ${ }_{N}^{P} \hat{\boldsymbol{s}}_{m}$ for the ship deck polygon $S$, RSD deck $D$, and wedge clamps $C L$ and $C D$ are unit vectors developed using the left-hand normal where the polygon vertices are defined in a clockwise order. The vertex positions are taken from the kinematic development captured in Equations 3.40, 3.42, 3.44, 
and 3.45. The ship deck is shown as an example in Figure 4.6.

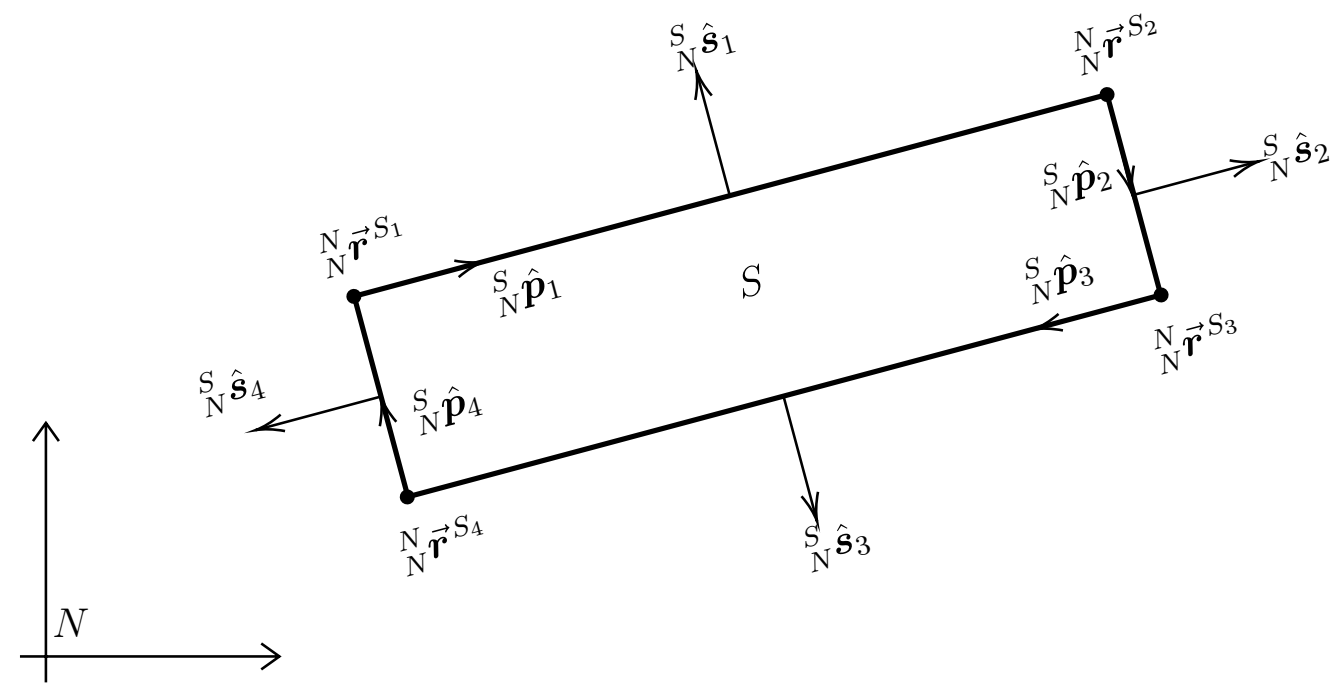

Figure 4.6: Surface normal axes ${ }_{N}^{S} \hat{\boldsymbol{s}}_{m}$ for the ship polygon $S$.

For $N_{v, P}$ vertices in a polygon, the polygon is composed of $N_{s, P}=N_{v, P}$ surfaces. Therefore the surface unit vectors for each polygon are

$$
\begin{aligned}
& { }_{N}^{S} \hat{\boldsymbol{s}}_{m}=\left[\begin{array}{cc}
0 & -1 \\
1 & 0
\end{array}\right]\left(\frac{\left({ }_{N}^{N} \overrightarrow{\boldsymbol{r}}^{S_{m+1}}-{ }_{N}^{N} \overrightarrow{\boldsymbol{r}}^{S_{m}}\right)}{\left\|_{N}^{N} \boldsymbol{r}^{S_{m+1}}-{ }_{N}^{N} \overrightarrow{\boldsymbol{r}}^{S_{m}}\right\|}\right) \quad m=1, \ldots, N_{v, S} \\
& { }_{N}^{D} \hat{\boldsymbol{s}}_{m}=\left[\begin{array}{cc}
0 & -1 \\
1 & 0
\end{array}\right]\left(\frac{\left({ }_{N}^{N} \overrightarrow{\boldsymbol{r}}^{D_{m+1}}-{ }_{N}^{N} \overrightarrow{\boldsymbol{r}}^{D_{m}}\right)}{\left\|{ }_{N}^{N} \overrightarrow{\boldsymbol{r}}^{D_{m+1}}-{ }_{N}^{N} \overrightarrow{\boldsymbol{r}}^{D_{m}}\right\|}\right) \quad m=1, \ldots, N_{v, D} \\
& { }_{N}^{C L} \hat{\boldsymbol{s}}_{m}=\left[\begin{array}{cc}
0 & -1 \\
1 & 0
\end{array}\right]\left(\frac{\left({ }_{N}^{N} \overrightarrow{\boldsymbol{r}}^{C L_{m+1}}-{ }_{N}^{N} \overrightarrow{\boldsymbol{r}}^{C L_{m}}\right)}{\|{ }_{N}^{N} \overrightarrow{\boldsymbol{r}}^{C L_{m+1}}-{ }_{N}^{N} \overrightarrow{\boldsymbol{r}}^{C L_{m} \|}}\right) \quad m=1, \ldots, N_{v, C L} \\
& { }_{N}^{C R} \hat{\boldsymbol{s}}_{m}=\left[\begin{array}{cc}
0 & -1 \\
1 & 0
\end{array}\right]\left(\frac{\left({ }_{N}^{N} \overrightarrow{\boldsymbol{r}}^{C R_{m+1}}-{ }_{N}^{N} \overrightarrow{\boldsymbol{r}}^{C R_{m}}\right)}{\left\|{ }_{N}^{N} \overrightarrow{\boldsymbol{r}}^{C R_{m+1}}-{ }_{N}^{N} \overrightarrow{\boldsymbol{r}}^{C R_{m}}\right\|}\right) \quad m=1, \ldots, N_{v, C R}
\end{aligned}
$$

For $m=N_{v, P}$, the superscript $m+1$ equals 1 . It is also important to note that the expression in parenthesis in equations 4.32 through 4.35 are the surface parallel unit vectors (or surface parallel axes) ${ }_{N}^{P} \hat{\boldsymbol{p}}_{m}$.

The contact detection algorithm is developed in detail in Section 5.2.2 of Chapter 5. Nevertheless, assuming surface ${ }^{P} s_{m}$ is the surface where node $n_{j_{c}}$ has come into contact with polygon $P$, the relationship in Equation 4.36 is satisfied.

$$
{ }_{N}^{N} \overrightarrow{\boldsymbol{r}}^{n_{j_{c}}} \cdot{ }_{N}^{P} \hat{\boldsymbol{s}}_{m} \leq \max \left({ }_{N}^{N} \boldsymbol{r}^{P_{m}} \cdot{ }_{N}^{P} \hat{\boldsymbol{s}}_{m}\right), \quad m=1, \ldots, N_{v, P}
$$

or simply

$$
{ }_{N}^{N} \boldsymbol{r}^{n_{j_{c}}} \cdot{ }_{N}^{P} \hat{\boldsymbol{s}}_{m} \leq\left({ }_{N}^{N} \boldsymbol{r}^{P_{m}} \cdot{ }_{N}^{P} \hat{\boldsymbol{s}}_{m}\right)
$$


since the vertex ${ }_{N}^{N} \overrightarrow{\boldsymbol{r}}^{P_{m}}$ is the maximum value as it defines surface ${ }^{P} s_{m}$ evaluated for contact.

Equation 4.36 and 4.37 imply penetration of the contacting node $n_{j_{c}}$ into surface ${ }^{P} s_{m}$ since the positive direction of ${ }_{N}^{P} \hat{\boldsymbol{s}}_{m}$ is outwards. This is the basis for contact response.

\section{Contact Response}

The normal and parrallel vectors generated in the SAT model, can be re-used to generate the oriented dynamic response due to collision. The polygon vertices and contact node points can be projected to surface normal axis ${ }_{N}^{P} \hat{\boldsymbol{s}}_{m}$ such that a penalty function can be used to apply a contact restitution force normal to surface ${ }^{P} s_{m}$. In other words, a penalty function is used to generate normal forces when in contact with surface ${ }^{P} s_{m}$. The relative sliding velocity of a contact node along ${ }^{P} s_{m}$ can be used to generate a sliding friction force.

Though any penalty functions can be used for collision restitution, a virtual linear spring-damper penalty function is used in the planar case of SRAMSS to apply a restitution force which is proportional to penetration depth and penetration velocity, as shown in Figure 4.7. The penalty function is expressed

$$
{ }_{n_{j_{c}}} F^{P}= \begin{cases}-K_{p} \Delta_{p}-C_{p} \dot{\Delta}_{p}, & { }_{N}^{N} \overrightarrow{\boldsymbol{r}}^{n_{j_{c}}} \cdot{ }_{N}^{P} \hat{\boldsymbol{s}}_{m} \leq\left({ }_{N}^{N} \boldsymbol{\boldsymbol { r }}^{P_{m}} \cdot{ }_{N}^{P} \hat{\boldsymbol{s}}_{m}\right) \\ 0, & { }_{N}^{N} \overrightarrow{\boldsymbol{r}}^{n_{j_{c}}} \cdot{ }_{N}^{P} \hat{\boldsymbol{s}}_{m}>\left({ }_{N}^{N} \overrightarrow{\boldsymbol{r}}^{P_{m}} \cdot{ }_{N}^{P} \hat{\boldsymbol{s}}_{m}\right)\end{cases}
$$

where ${ }_{\hat{\boldsymbol{s}}_{m}}^{n_{j_{c}}} F^{P}$ is the restitution force applied by polygon $P$ on node $n_{j_{c}}$ along the positive direction of ${ }_{N}^{P} \hat{\boldsymbol{s}}_{m}, \Delta_{p}$ is the surface penetration depth of $n_{j_{c}}$ along ${ }_{N}^{P} \hat{\boldsymbol{s}}_{m}$, and $\dot{\Delta}_{p}$ is the relative surface penetration velocity of $n_{j_{c}}$ along ${ }_{N}^{P} \hat{\boldsymbol{s}}_{m}$. The values $K_{p}$ and $C_{p}$ are the penetration stiffness and damping coefficients respectively. The surface penetration depth is given as the difference between vertex ${ }_{N}^{N} \overrightarrow{\boldsymbol{r}}^{P_{m}}$ and contact node ${ }_{N}^{N} \overrightarrow{\boldsymbol{r}}^{n_{j_{c}}}$ as projected to ${ }_{N}^{P} \hat{\boldsymbol{s}}_{m}$, expressed as

$$
\Delta_{p}=\left({ }_{N}^{N} \overrightarrow{\boldsymbol{r}}^{n_{j_{c}}}-{ }_{N}^{N} \overrightarrow{\boldsymbol{r}}^{P_{m}}\right) \cdot{ }_{N}^{P} \hat{\boldsymbol{s}}_{m}
$$

Since the vertex ${ }_{N}^{N} \overrightarrow{\boldsymbol{r}}^{P_{m}}$ belongs to surface ${ }^{P} s_{m}$, it serves as the surface reference point along ${ }_{N}^{P} \hat{\boldsymbol{s}}_{m}$ to calculate penetration depth.

For the relative penetration velocity, a physical reference point on surface ${ }^{P} s_{m}$ is required. This point is defined to be ${ }_{N}^{s_{m}} \overrightarrow{\boldsymbol{r}}^{n_{j_{c}}}$, the position of node $n_{j_{c}}$ as projected 


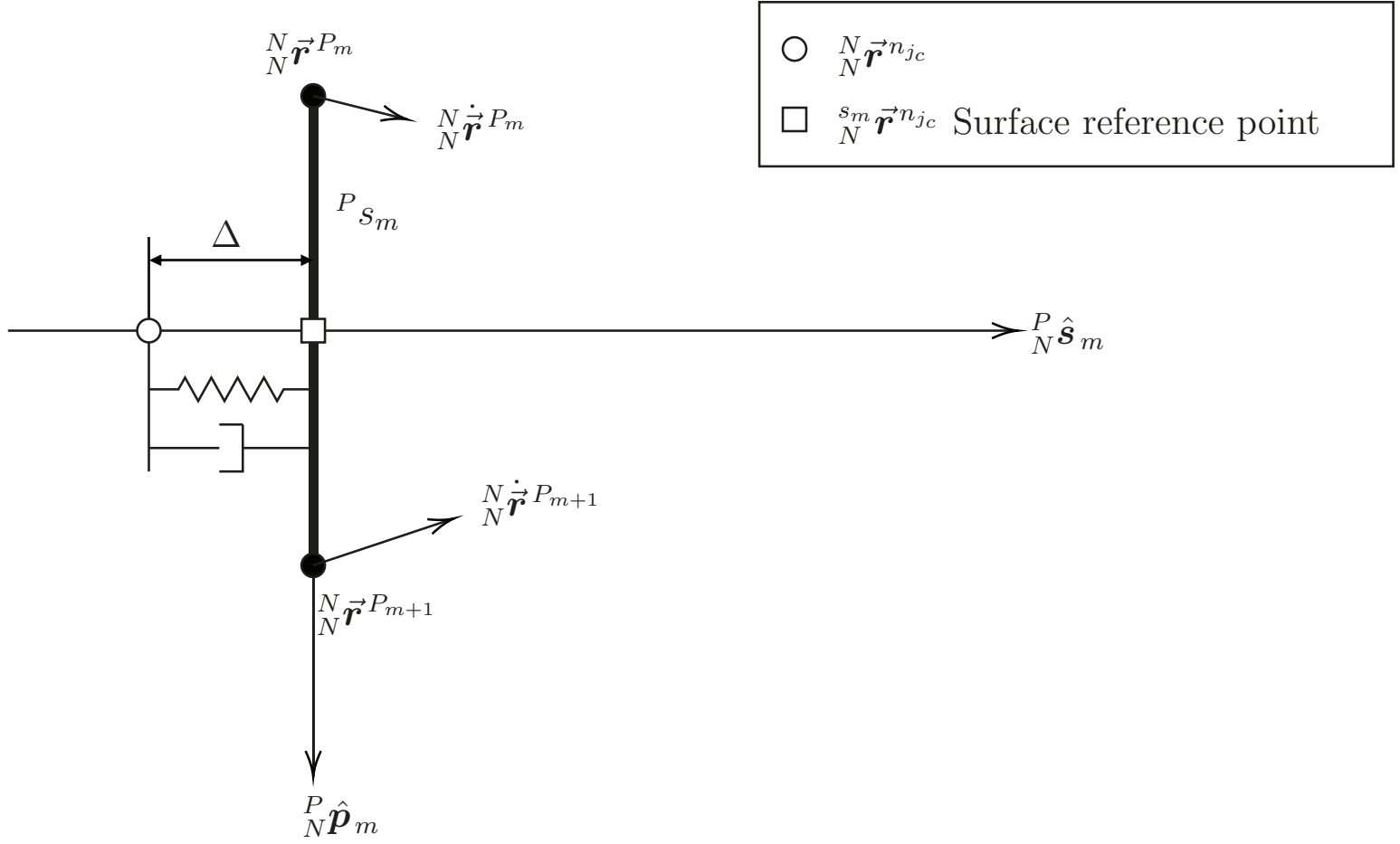

Figure 4.7: Surface penetration penalty function diagram for contact node $n_{j_{c}}$ in collision with surface ${ }^{P} s_{m}$

to the surface ${ }^{P} s_{m}$ with respect to the inertial frame, and expressed in the inertial frame. In terms of the vertices of polygon $P,{ }_{N}^{s_{m}} \overrightarrow{\boldsymbol{r}}^{n_{j_{c}}}$ is

$$
{ }_{N}^{s_{m}} \overrightarrow{\boldsymbol{r}}^{n_{j_{c}}}={ }_{N}^{N} \overrightarrow{\boldsymbol{r}}^{P_{m}}+\left[\left({ }_{N}^{N} \boldsymbol{\boldsymbol { r }}^{n_{j_{c}}}-{ }_{N}^{N} \overrightarrow{\boldsymbol{r}}^{P_{m}}\right) \cdot{ }_{N}^{P} \hat{\boldsymbol{p}}_{m}\right]{ }_{N}^{P} \hat{\boldsymbol{p}}_{m}
$$

Then, the velocity at the projected point ${ }_{N}^{s_{m}} \overrightarrow{\boldsymbol{r}}^{n_{j_{c}}}$ is

$$
{ }_{N}^{s_{m}} \dot{\overrightarrow{\boldsymbol{r}}} n_{j_{c}}={ }_{N}^{N} \dot{\overrightarrow{\boldsymbol{r}}} P_{m}+\left(\frac{\left\|{ }_{N}^{s_{m}} \overrightarrow{\boldsymbol{r}}^{n_{j c}}-{ }_{N}^{N} \overrightarrow{\boldsymbol{r}}^{P_{m}}\right\|}{\left\|{ }_{N}^{N} \overrightarrow{\boldsymbol{r}}^{P_{m+1}}-{ }_{N}^{N} \overrightarrow{\boldsymbol{r}}^{P_{m}}\right\|}\right)\left({ }_{N}^{N} \dot{\overrightarrow{\boldsymbol{r}}}^{P_{m+1}}-{ }_{N}^{N} \dot{\overrightarrow{\boldsymbol{r}}} P_{m}\right)
$$

Now, the relative penetration velocity can be determined similarly to the penetration depth by calculating relative velocity between the contact node and its surface projection as

$$
\dot{\Delta}_{p}=\left({ }_{N}^{N} \dot{\overrightarrow{\boldsymbol{r}}} n_{j_{c}}-{ }_{N}^{s_{m}} \dot{\overrightarrow{\boldsymbol{r}}} n_{j_{c}}\right) \cdot{ }_{N}^{P} \hat{\boldsymbol{s}}_{m}
$$

The equations required for the surface normal forces have been developed. Development of the equations governing contact frictional forces remain.

A frictional slider model is employed to generate friction forces along the penetrated surface ${ }^{P} s_{m}$. Such models require a reference point, an additional statevariable, from which friction model quantities can be calculated. In this work, these 
reference points are called 'Object Contact Points' (OCPs). The behaviour of OCPs is governed by specific rules:

- There is one OCP ${ }_{N}^{P} \overrightarrow{\boldsymbol{o}}^{n_{j_{c}}}$ per contact node $n_{j_{c}}$ per defined polygon $P$. This enables the generation of dynamic responses for simultaneous contact of multiple polygons.

- While not in contact with polygon $P$, the OCP for $n_{j_{c}}$ is determined by the projection of ${ }_{N}^{N} \overrightarrow{\boldsymbol{r}}^{n_{j_{c}}}$ onto the closest surface ${ }^{P} s_{m}$.

- Once in contact with polygon $P$, the OCP is determined to be the initial point of contact with polygon $P$.

- During contact with polygon $P$, the OCP's position on the surface ${ }^{P} s_{m}$ is only shifted when the maximum allowable friction force is exceeded. the OCP is shifted to generate a frictional force exactly equal to the maximum allowable value, hence the frictional slider.

Outside of contact with polygon $P$, the OCP located as

$$
{ }_{N}^{P} \overrightarrow{\boldsymbol{o}}^{n_{j_{c}}}={ }_{N}^{N} \overrightarrow{\boldsymbol{r}}^{P_{m}}+\left[\left({ }_{N}^{N} \overrightarrow{\boldsymbol{r}}^{n_{j_{c}}}-{ }_{N}^{N} \overrightarrow{\boldsymbol{r}}^{P_{m}}\right) \cdot{ }_{N}^{P} \hat{\boldsymbol{p}}_{m}\right]{ }_{N}^{P} \hat{\boldsymbol{p}}_{m}
$$

It is necessary to express the position of the OCP relative to the polygon frame $P$. For a polygon undergoing transient motion, the OCP must follow with the polygon, yet must not shift along the surface since the maximum friction value may not have been exceeded. Thus, the OCP must follow the motion of the polygon between shifts during contact.

$$
{ }_{P}^{P} \overrightarrow{\boldsymbol{o}}^{n_{j_{c}}}=\left[\boldsymbol{T}_{N \leftarrow P}\right]^{T}\left({ }_{N}^{P} \overrightarrow{\boldsymbol{o}}^{n_{j_{c}}}-{ }_{N}^{N} \overrightarrow{\boldsymbol{r}}^{P}\right)
$$

where $\left[\boldsymbol{T}_{N \leftarrow P}\right]^{T}$ is the inverse of the transformation from the polygon frame $P$ through the chain of polygon frames to the inertial frame, and ${ }_{N}^{N} \overrightarrow{\boldsymbol{r}}^{P}$ is the position of polygon frame $P$ with respect to the inertial frame, and expressed in the inertial frame. For oriented dynamic response, since all other vectors are with respect to, and expressed in the inertial frame, the general expression for an OCP is

$$
{ }_{N}^{P} \overrightarrow{\boldsymbol{o}}^{n_{j_{c}}}={ }_{N}^{N} \overrightarrow{\boldsymbol{r}}^{P}+\left[\boldsymbol{T}_{N \leftarrow P}\right]_{P}^{P} \overrightarrow{\boldsymbol{o}}^{n_{j_{c}}}
$$

With the OCPs defined, the frictional-slider model can be developed. The LuGre friction model is adapted for use in SRAMSS. This model is capable of modelling static, dynamic, and viscous friction, and pre-sliding micro-displacements. For this work, viscous friction is not modelled as it is not conceptually applicable to the UAS dynamic interface problem. The remaining frictional terms are modelled by 
representing surface asperities with a 'bristle' model as shown in Figure 4.8. The deflection $\Delta_{f}$, and rate of deflection $\dot{\Delta}_{f}$ of the bristle are the force generating elements of the LuGre model.

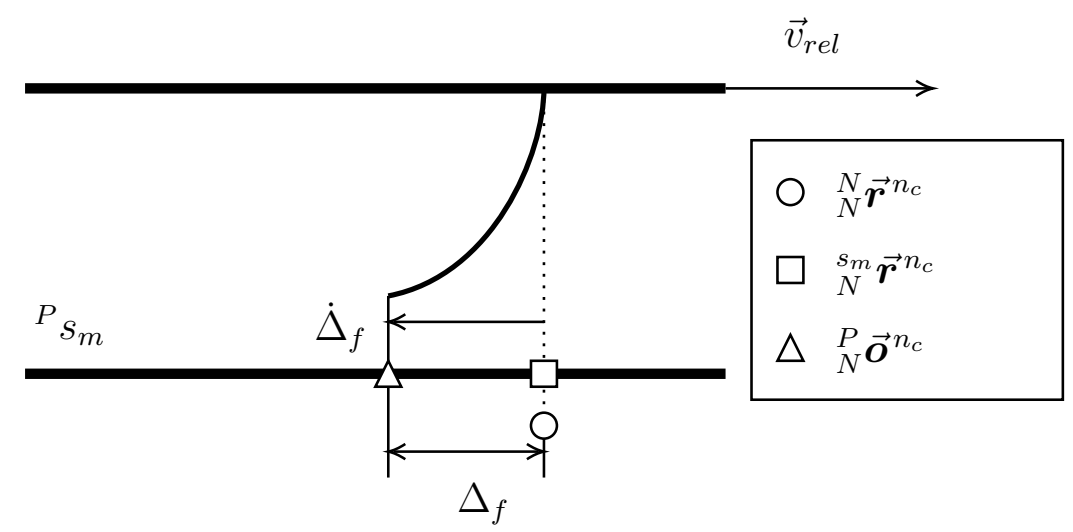

Figure 4.8: LuGre friction bristle model.

The deflection of the bristle $\Delta_{f}$ is determined by the distance between the OCP and the contacting node projected to surface ${ }^{P} s_{m}$ such that

$$
\Delta_{f}=\left({ }_{N}^{P} \overrightarrow{\boldsymbol{o}}^{n_{j_{c}}}-{ }_{N}^{s_{m}} \overrightarrow{\boldsymbol{r}}^{n_{j_{c}}}\right) \cdot{ }_{N}^{P} \hat{\boldsymbol{p}}_{m}
$$

and the rate of deflection of the bristle $\dot{\Delta}_{f}$ is

$$
\dot{\Delta}_{f}=-v_{r e l}\left(1-\frac{\left|\Delta_{f}\right|}{\Delta_{f, \max }}\right)
$$

where $v_{r e l}$, is the relative velocity of $n_{j_{c}}$ with respect to ${ }_{N}^{P} \overrightarrow{\boldsymbol{o}}^{n_{j_{c}}}$, which represents the sliding velocity along ${ }^{P} s_{m}$. Equation 4.47 ensures that the rate of bristle deflection $\dot{\Delta}_{f} \equiv 0$ at maximum bristle deflection $\Delta_{f, \max }$. The relative sliding velocity $v_{r e l}$ is calculated

$$
\begin{aligned}
& v_{r e l}=\left({ }_{N}^{N} \dot{\overrightarrow{\boldsymbol{r}}}^{n_{j_{c}}}-{ }_{N}^{P} \dot{\overrightarrow{\boldsymbol{o}}}^{n_{j_{c}}}\right) \cdot{ }_{N}^{P} \hat{\boldsymbol{p}}_{m} \\
& =\left({ }_{N}^{N} \dot{\overrightarrow{\boldsymbol{r}}} n_{j_{c}}-\left[{ }_{N}^{N} \dot{\overrightarrow{\boldsymbol{r}}} P_{m}+\frac{\left\|{ }_{N}^{P} \dot{\overrightarrow{\boldsymbol{o}}}^{n_{j_{c}}}-{ }_{N}^{N} \overrightarrow{\boldsymbol{r}}^{P_{m}}\right\|}{\left\|{ }_{N}^{N} \overrightarrow{\boldsymbol{r}}^{P_{m+1}}-{ }_{N}^{N} \overrightarrow{\boldsymbol{r}}^{P_{m}}\right\|}\left({ }_{N}^{N} \dot{\overrightarrow{\boldsymbol{r}}} P_{m+1}-{ }_{N}^{N} \dot{\overrightarrow{\boldsymbol{r}}} P_{m}\right)\right]\right) \cdot{ }_{N}^{P} \hat{\boldsymbol{p}}_{m}
\end{aligned}
$$

The maximum deformation of the bristle $\Delta_{f, \max }$ is governed by a Stribeck curve which dictates the dominance of static $F_{s}$ or coulomb $F_{d}$ friction forces as a function of relative sliding velocity $v_{r e l}[40]$. The Stribeck curve used for the LuGre model is

$$
\Delta_{f, \max }=\frac{F_{d}+\left(F_{s}-F_{d}\right)^{-\left(\left|v_{r e l} / v_{s}\right|\right)^{2}}}{K_{f}}
$$


with $v_{s}$ the Stribeck velocity threshold under which static friction dominates, and $K_{f}$ is the stiffness of the bristle[40]. For $\Delta_{f} \geq \Delta_{f, \max }$ then $\Delta_{f}=\Delta_{f, \max }$ in Equation 4.47.

Static and coulomb friction values are obtained from the normal force ${ }_{\hat{\boldsymbol{s}}_{m}}^{n_{j_{c}}} F^{P}$ calculated in Equation 4.38, such that

$$
\begin{aligned}
& F_{s}=\mu_{s}{ }_{\hat{\boldsymbol{s}}_{m}}^{n_{j_{c}}} F^{P} \\
& F_{d}=\mu_{d}{ }_{\hat{\boldsymbol{s}}_{m}}^{n_{j_{c}}} F^{P}
\end{aligned}
$$

where $\mu_{s}$ and $\mu_{d}$ are the static and dynamic coefficients of friction, respectively.

Ultimately the friction force generated on contact node $n_{j_{c}}$ sliding along surface $P_{s_{m}}$ is

$$
{ }_{\hat{\boldsymbol{p}}_{m}}^{n_{j_{c}}} F^{P}= \begin{cases}K_{f} \Delta_{f}-C_{f r i c} \dot{\Delta}_{f}, & \Delta_{f}<\Delta_{f, \max } \\ K_{f} \Delta_{f, \max }, & \Delta_{f} \geq \Delta_{f, \max }\end{cases}
$$

where $C_{f}$ is the bristle damping coefficient.

The complete set of forces which constitute the oriented dynamic response are

\begin{tabular}{|c|c|c|c|c|c|c|}
\hline & Direction & Quantity & \multicolumn{4}{|c|}{ Governing Formula } \\
\hline Normal Force & ${ }_{N}^{P} \hat{\boldsymbol{s}}_{m}$ & ${ }_{\hat{\boldsymbol{s}}_{m}}^{n_{j_{c}}} F^{P}$ & $=K_{p}$ & $\Delta_{p}$ & $-C_{p}$ & $\dot{\Delta}_{p}$ \\
\hline Equation Index & & $(4.38)$ & & $(4.39)$ & & $(4.42)$ \\
\hline Friction Force & ${ }_{N}^{P} \hat{\boldsymbol{p}}_{m}$ & $\begin{array}{l}n_{j_{c}} \\
\hat{\boldsymbol{p}}_{m}\end{array} F^{P}$ & $=K_{f}$ & $\Delta_{f}$ & $-C_{f}$ & $\dot{\Delta}_{f}$ \\
\hline Equation Index & & $(4.52)$ & & $(4.46)^{1}$ & & $(4.47)$ \\
\hline
\end{tabular}
presented in Table 4.1 .

Table 4.1: Contact forces summary

${ }^{1}$ : At maximum bristle deflection $\Delta_{f}=\Delta_{f, \max }, \dot{\Delta}_{f}=0$

The normal and friction forces are scalar values oriented along their respective axes. The complete oriented dynamic response on contact node $n_{j_{c}}$ due to contact with polygon $P$ on surface ${ }^{A} S_{m}$ is then

$$
{ }_{N}^{n_{j_{c}}} \overrightarrow{\boldsymbol{F}}^{P}={ }_{N}^{P} \hat{\boldsymbol{s}}_{m}{ }_{\hat{\boldsymbol{s}}_{m}}^{n_{j_{c}}} F^{P}+{ }_{N}^{P} \hat{\boldsymbol{p}}_{m}{ }_{\hat{\boldsymbol{p}}_{m}}^{n_{j_{c}}} F^{P}
$$

The external nodal forces vector, and subsequent assembly into the governing equations of Kane's method require that the forces acting on a body be expressed in the body's frame. therefore Equation 4.53, expressed in $n_{j_{c}}$ 's frame, becomes:

$$
\begin{aligned}
& { }_{n_{j_{c}}}^{n_{j_{c}}} \overrightarrow{\boldsymbol{F}}^{P}=\left[\boldsymbol{T}_{n_{j_{c}} \leftarrow N}\right]_{N}^{n_{j_{c}}} \overrightarrow{\boldsymbol{F}}^{P}
\end{aligned}
$$

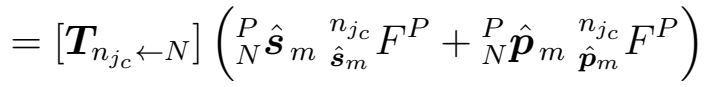


In the SRAMSS environment, there are four polygons representing the ship deck $S$, RSD deck $D$, and wedge clamps $C L$ and $C R$. The external nodal forces vector due to contact ${ }_{n}^{n} \boldsymbol{F}_{k}^{c}$ is assembled from the contact force contributions from each polygon on all contact nodes:

$$
{ }_{n}^{n} \boldsymbol{F}_{k}^{c}=\left\{\begin{array}{c}
{ }_{n_{1}} \overrightarrow{\boldsymbol{F}}^{c} \\
n_{1} \\
{ }_{n_{1}} \overrightarrow{\boldsymbol{M}}^{c} \\
\vdots \\
n_{1} \\
n_{n_{j}} \overrightarrow{\boldsymbol{F}}^{c} \\
{ }_{n_{j}} \overrightarrow{\boldsymbol{M}}^{c}
\end{array}\right\}
$$

where

$$
\begin{aligned}
&\left\{\begin{array}{c}
{ }_{n_{j}} \overrightarrow{\boldsymbol{F}}^{c} \\
n_{j} \\
n_{j} \overrightarrow{\boldsymbol{M}}^{c}
\end{array}\right\}=\left\{\begin{array}{c}
n_{n_{j}} \overrightarrow{\boldsymbol{F}}^{S}+{ }_{n_{j}}^{n_{j}} \overrightarrow{\boldsymbol{F}}^{D}+{ }_{n_{j}}^{n_{j}} \overrightarrow{\boldsymbol{F}}^{C L}+{ }_{n_{j}}^{n_{j}} \overrightarrow{\boldsymbol{F}}^{C R} \\
0
\end{array}\right\} \\
& j=1, \ldots, N_{n} \forall j=j_{c}
\end{aligned}
$$

otherwise

$$
\left\{\begin{array}{c}
n_{j} \overrightarrow{\boldsymbol{F}}^{c} \\
n_{j} \\
n_{j} \\
n_{j} \overrightarrow{\boldsymbol{M}}^{c}
\end{array}\right\}=\left\{\begin{array}{l}
0 \\
0
\end{array}\right\}, \quad j=1, \ldots, N_{n} \forall j \neq j_{c}
$$

\subsection{Aerodynamic Models}

Inherent to the system being simulated, aerodynamic forces acting-on, or generated by the aircraft are phenomena that must be considered. There are two major aerodynamic phenomena which are modelled in SRAMSS. The first being aerodynamic drag forces exerted on the airframe due to relative wind velocity. The second being forces and moments acting on the airframe due to the uneven thrust generated by advancing and retreating rotor blades in apparent wind. Additionally, due to the spin of the rotor blades, there are gyroscopic effects acting on the aircraft body. However, for the planar case they are not applicable.

In this work, there is no characterization of the flow field in the aircraft landing zone. This simplification eliminates the need to characterize the influence of the ship's superstructure, rotor downwash, rotor wake, and other non-uniform flow behaviours. The following sections develop the aerodynamic models present in SRAMSS. 


\subsubsection{Aerodynamic Body Drag Forces}

Similar to DYNAFACE ${ }^{\circledR}$ [22] and SSMASH [21], SRAMSS models aerodynamic drag forces due to apparent wind acting at the airframe by using equivalent projected flatplate areas $\boldsymbol{A}$ which allows simplifications in the aerodynamic drag equations by treating the drag coefficient as unity [52]

$$
\boldsymbol{A}_{e q}=\left\{\begin{array}{c}
A_{e q, x} \\
A_{e q, y} \\
A_{e q, z}
\end{array}\right\}
$$

The aerodynamic body drag forces are applied at the airframe's centre of pressure (CP). Therefore, wind velocity relative to this point is required. Orientation of the wind vector is assumed to be uniform across the ship deck. First, consider the wind velocity in the inertial frame:

$$
{ }_{N}^{N} \overrightarrow{\boldsymbol{v}}^{W}=\left\{\begin{array}{l}
v_{\text {wind,x }} \\
v_{\text {wind,y }} \\
v_{\text {wind, }, z}
\end{array}\right\}
$$

Next, the velocity of the airframe's CP with respect to the inertial frame is required. The CP is assumed to be at a fixed distance ${ }_{H}^{H} \overrightarrow{\boldsymbol{r}}^{C P}$ from the airframe's CoG, the $H$-frame origin. The CP velocity is then expressed as:

$$
\begin{aligned}
& { }_{N}^{N} \overrightarrow{\boldsymbol{v}}^{C P}=\frac{\mathrm{d}}{\mathrm{d} t}\left({ }_{N}^{N} \overrightarrow{\boldsymbol{r}}^{C P}\right) \\
& =\frac{\mathrm{d}}{\mathrm{d} t}\left({ }_{N}^{N} \overrightarrow{\boldsymbol{r}}^{H}\right)+\frac{\mathrm{d}}{\mathrm{d} t}\left(\left[\boldsymbol{T}_{N \leftarrow H}\right]_{H}^{H} \overrightarrow{\boldsymbol{r}}^{C P}\right) \\
& ={ }_{N}^{N} \dot{\overrightarrow{\boldsymbol{r}}}^{H}+\left[\dot{\boldsymbol{T}}_{N \leftarrow H}\right]_{H}^{H} \overrightarrow{\boldsymbol{r}}^{C P}+\left[\boldsymbol{T}_{N \leftarrow H}\right]_{H}^{H} \dot{\overrightarrow{\boldsymbol{r}}}^{C P} \\
& ={ }_{N}^{N} \dot{\overrightarrow{\boldsymbol{r}}}^{H}+\left[\boldsymbol{T}_{N \leftarrow H}\right]\left[\widetilde{N_{H}^{N \overrightarrow{\boldsymbol{\omega}}^{H}}}\right]_{H}^{H} \overrightarrow{\boldsymbol{r}}^{C P}+\left[\boldsymbol{T}_{N \leftarrow H}\right]_{H}^{H} \dot{\overrightarrow{\boldsymbol{p}}} C \boldsymbol{P}^{-} \\
& ={ }_{N}^{N} \dot{\overrightarrow{\boldsymbol{r}}}^{H}+\left[\boldsymbol{T}_{N \leftarrow H}\right]\left[\widetilde{{ }_{H}^{N \overrightarrow{\boldsymbol{\omega}} H}}\right]_{H}^{H} \overrightarrow{\boldsymbol{r}}^{C P}
\end{aligned}
$$

Equations 4.59 and 4.60 are both measured relative to the inertial frame, and expressed in the inertial frame. Therefore the wind velocity relative to the $\mathrm{CP}$ can be expressed:

$$
\begin{aligned}
{ }_{N}^{C P} \overrightarrow{\boldsymbol{v}}^{W} & ={ }_{N}^{N} \overrightarrow{\boldsymbol{v}}^{W}-{ }_{N}^{N} \overrightarrow{\boldsymbol{v}}^{C P} \\
& ={ }_{N}^{N} \overrightarrow{\boldsymbol{v}}^{W}-{ }_{N}^{N} \dot{\overrightarrow{\boldsymbol{r}}}^{H}+\left[\boldsymbol{T}_{N \leftarrow H}\right]\left[\widetilde{{ }_{H}^{N} \overrightarrow{\boldsymbol{\omega}}^{H}}\right]_{H}^{H} \overrightarrow{\boldsymbol{r}}^{C P}
\end{aligned}
$$


Body forces acting on the airframe in $H$ require expression of Equation 4.61 in the $H$ frame. Therefore Equation 4.61 becomes

$$
{ }_{H}^{C P} \overrightarrow{\boldsymbol{v}}^{W}=\left[\boldsymbol{T}_{H \leftarrow N}\right]_{N}^{N} \overrightarrow{\boldsymbol{v}}^{W}-\left[\boldsymbol{T}_{H \leftarrow N}\right]_{N}^{N} \dot{\overrightarrow{\boldsymbol{r}}}^{H}+\left[\widetilde{{ }_{H}^{N} \overrightarrow{\boldsymbol{\omega}}^{H}}\right]_{H}^{H} \overrightarrow{\boldsymbol{r}}^{C P}
$$

Then, the aerodynamic body drag forces and moments acting on the helicopter can be expressed:

$$
\begin{aligned}
{ }_{H}^{H} \overrightarrow{\boldsymbol{F}}^{W} & =\left.\frac{1}{2} \rho_{a i r} \boldsymbol{A}_{e q} \circ{ }_{H}^{C P} \overrightarrow{\boldsymbol{v}}^{W} \circ\right|_{H} ^{C P} \overrightarrow{\boldsymbol{v}}^{W} \mid \\
{ }_{H}^{H} \overrightarrow{\boldsymbol{M}}^{W} & =\left[{ }_{H}^{H} \overrightarrow{\boldsymbol{r}}^{C P}\right]_{H}^{H} \overrightarrow{\boldsymbol{F}}^{W}
\end{aligned}
$$

where ' $O$ ' is the Hadamard operator denoting element-wise multiplication, and the square of velocity is taken to be the value of velocity multiplied by its absolute value to retain vector orientation.

\subsubsection{Aerodynamic Rotor Disc Forces}

Where DYNAFACE ${ }^{\circledR}$ models rotor thrust during landing transients and induced rotor forces from free-spinning rotor blades separately [22], SRAMSS unifies these two types of forces by implementing a Blade Element Model (BEM) which can characterize the thrust forces and moments on the aircraft at any point [43]. Blade element modelling is chosen since it is versatile for representing any type of rotor blade and airfoil shape by discretization of the rotor blade, and provides accuracy to the extent desired by inclusion of different rotor blade phenomena.

Similar to the discretization of the landing gear through the DFE model, the BEM discretizes the complex rotor blade profile into $N_{e}$ discrete rotor blade elements (RBEs). The $h$ th RBE $e_{h}$ is located at radial position $r_{h}$, azimuth $\psi$, and having width $\mathrm{d} r$, as illustrated in Figure 4.9.

The local airflow over an RBE is obtained to calculate the element's lift and moment. Then, azimuth-averaged elemental thrust and moment are calculated by discretization of the azimuth into $N_{\psi}$ azimuth sectors $\psi_{l}$ as shown in the BEM mesh of Figure 4.10. The averaged element values are then summed over the length of the rotor blade and multiplied by the number of rotor blades $N_{b}$ to obtain the total thrust and moment generated by the rotor disc in apparent wind. The BEM in SRAMSS is an azimuth-averaged actuator disc approach which eliminates the need to propagate a state variable for the orientation of the rotor blades. 


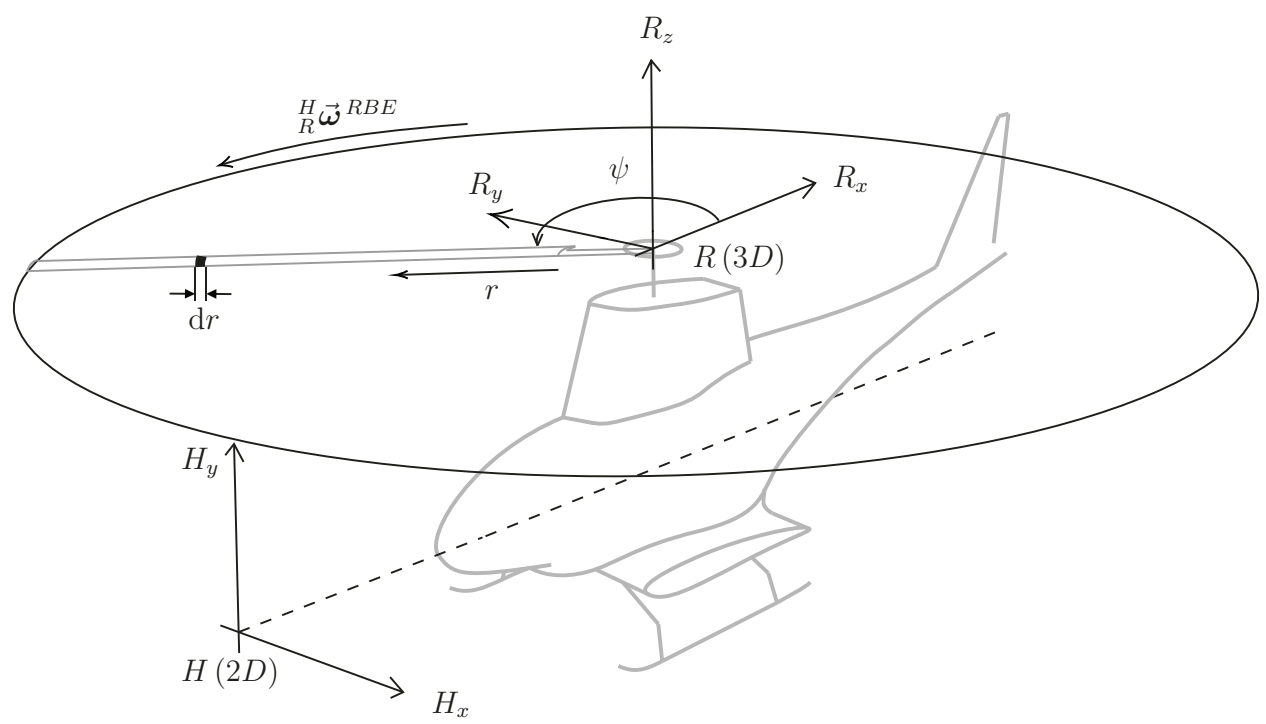

Figure 4.9: Rotor disc blade element model for generic aircraft. $(H$ frame moved forward for clarity)

Additionally, consideration is also made for the rotor blade inner and outer effective radii $R_{i}$ and $R_{o}$, outside of which the blade is considered to not be generating any significant lift. These effective radii are expressed as percentages of the physical radius of the rotor blade $R_{b}$.

The complex rotor blade profile has radially-varying parameters which affect the generation of lift. As a result, for the BEM, each RBE is prescribed specific parameter values. These radially varying parameters include:

- chord length $c_{h}$;

- airfoil thickness to chord ratio $t / c_{h}$;

- blade twist angle $\theta_{t_{h}}$;

- lift coefficient gradient $\frac{\mathrm{d} C_{L}}{\mathrm{~d} \alpha_{h l}}$ and zero-angle of attack lift coefficient $C_{L, \alpha_{0, h}}$;

- drag coefficient gradient $\frac{\mathrm{d} C_{D}}{\mathrm{~d} \alpha_{h l}}$ and zero-angle of attack lift coefficient $C_{D, \alpha_{0, h}}$.

Each RBE is also dependent on rotor disc prescribed parameters:

- collective pitch setting angle $\theta_{\text {coll }}$;

- maximum cyclic pitch setting angle $\theta_{c y c, \max }$ at azimuth $\psi_{\max }$.

To calculate the element thrust and moment for the $h$ th $\operatorname{RBE} e_{h}$ at $l$ th azimuth $\psi_{l}$, the element axial flow $v_{0_{h l}}$, tangential flow $v_{2_{h l}}$, and sectional flow $v_{1_{h l}}$ velocities illustrated in Figure 4.11 must be obtained.

To begin, the general expression of velocity of the $h$ th $\mathrm{RBE} e_{h}$ at $l$ th azimuth $\psi_{l}$ 


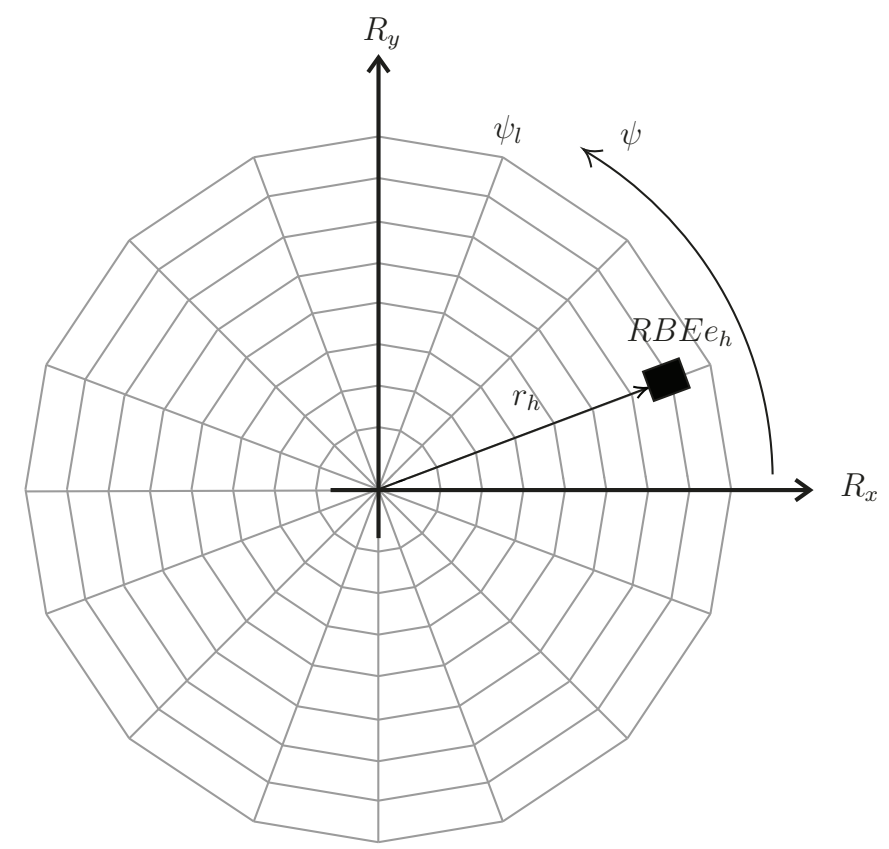

Figure 4.10: Rotor disc BEM mesh.

with respect to the inertial frame, and expressed in the inertial frame, is

$$
\begin{aligned}
& { }_{N}^{N} \overrightarrow{\boldsymbol{v}}^{e_{h} \psi_{l}}=\frac{\mathrm{d}}{\mathrm{d} t}\left({ }_{N}^{N} \overrightarrow{\boldsymbol{r}}^{e_{h} \psi_{l}}\right) \\
& =\frac{\mathrm{d}}{\mathrm{d} t}\left({ }_{N}^{N} \overrightarrow{\boldsymbol{r}}^{H}\right)+\frac{\mathrm{d}}{\mathrm{d} t}\left(\left[\boldsymbol{T}_{N \leftarrow H}\right]_{H}^{H} \overrightarrow{\boldsymbol{r}}^{R}\right)+\frac{\mathrm{d}}{\mathrm{d} t}\left(\left[\boldsymbol{T}_{N \leftarrow H}\right]\left[\boldsymbol{T}_{H \leftarrow R}\right]_{R}^{R} \overrightarrow{\boldsymbol{r}}^{e_{h} \psi_{l}}\right) \\
& ={ }_{N}^{N} \dot{\overrightarrow{\boldsymbol{r}}}^{H}+\left[\dot{\boldsymbol{T}}_{N \leftarrow H}\right]_{H}^{H} \overrightarrow{\boldsymbol{r}}^{R}+\left[\boldsymbol{T}_{N \leftarrow H}\right]_{H}^{H} \dot{\overrightarrow{\boldsymbol{r}}}^{R}+\left[\dot{\boldsymbol{T}}_{N \leftarrow H}\right]\left[\boldsymbol{T}_{H \leftarrow R}\right]_{R}^{R} \overrightarrow{\boldsymbol{r}}_{h}^{e_{h} \psi_{l}} \\
& +\left[\boldsymbol{T}_{N \leftarrow H}\right]\left[\dot{\boldsymbol{T}}_{H \leftarrow R}\right]_{R}^{R} \overrightarrow{\boldsymbol{r}}^{e_{h} \psi_{l}}+\left[\boldsymbol{T}_{N \leftarrow H}\right]\left[\boldsymbol{T}_{H \leftarrow R}\right]_{R}^{R} \dot{\overrightarrow{\boldsymbol{r}}} e_{h} \psi_{l} \\
& ={ }_{N}^{N} \dot{\overrightarrow{\boldsymbol{r}}} H+\left[\boldsymbol{T}_{N \leftarrow H}\right]\left[\widetilde{{ }_{H}^{N} \overrightarrow{\boldsymbol{\omega}}^{H}}\right]_{H}^{H} \overrightarrow{\boldsymbol{r}}^{R}+\left[\boldsymbol{T}_{N \leftarrow H}\right]_{H}^{H} \dot{\overrightarrow{\boldsymbol{r}}}^{\overbrace{}^{*}} \\
& \left.+\left[\boldsymbol{T}_{N \leftarrow H}\right] \widetilde{{ }_{H}^{N \overrightarrow{\boldsymbol{\omega}} H}}\right]\left[\boldsymbol{T}_{H \leftarrow R}\right]_{R}^{R} \overrightarrow{\boldsymbol{r}}^{e_{h} \psi_{l}} \\
& \left.+\left[\boldsymbol{T}_{N \leftarrow H}\right]\left[\boldsymbol{T}_{H \leftarrow R}\right] \widetilde{\left[{ }_{R}^{H} \overrightarrow{\boldsymbol{\omega}}^{R}\right.}\right]_{R}^{R} \overrightarrow{\boldsymbol{r}}^{e_{h} \psi_{l}}+\left[\boldsymbol{T}_{N \leftarrow H}\right]\left[\boldsymbol{T}_{H \leftarrow R}\right]_{R}^{R} \dot{\overrightarrow{\boldsymbol{p}} e \hbar \psi_{l}} \\
& \left.={ }_{N}^{N} \dot{\overrightarrow{\boldsymbol{r}}}^{H}+\left[\boldsymbol{T}_{N \leftarrow H}\right]\left[\widetilde{N_{H}^{N} \overrightarrow{\boldsymbol{\omega}}^{H}}\right]_{H}^{H} \overrightarrow{\boldsymbol{r}}^{R}+\left[\boldsymbol{T}_{N \leftarrow H}\right] \widetilde{{ }_{H}^{N \overrightarrow{\boldsymbol{\omega}}^{H}}}\right]\left[\boldsymbol{T}_{H \leftarrow R}\right]_{R}^{R} \overrightarrow{\boldsymbol{r}}^{e_{h} \psi_{l}} \\
& \left.+\left[\boldsymbol{T}_{N \leftarrow R}\right] \widetilde{\left[{ }_{R}^{H} \overrightarrow{\boldsymbol{\omega}}^{R}\right.}\right]_{R}^{R} \overrightarrow{\boldsymbol{r}}^{e_{h} \psi_{l}}, \quad h=1, \ldots, N_{e}, \quad l=1, \ldots, N_{\psi}
\end{aligned}
$$

where ${ }_{H}^{H} \boldsymbol{r}^{R}$ is the fixed position of the rotor disc frame $R$ with respect to the airframe CoG, ${ }_{R}^{R} \overrightarrow{\boldsymbol{r}} e_{h} \psi_{l}$ is the position of the $h$ th RBE $e_{h}$ on the rotor disc at azimuth $\psi_{l}$ and blade radius $r_{h}$, and ${ }_{R}^{H} \overrightarrow{\boldsymbol{\omega}}^{R}$ is the rotor disc RPM. As with the aerodynamic body drag 

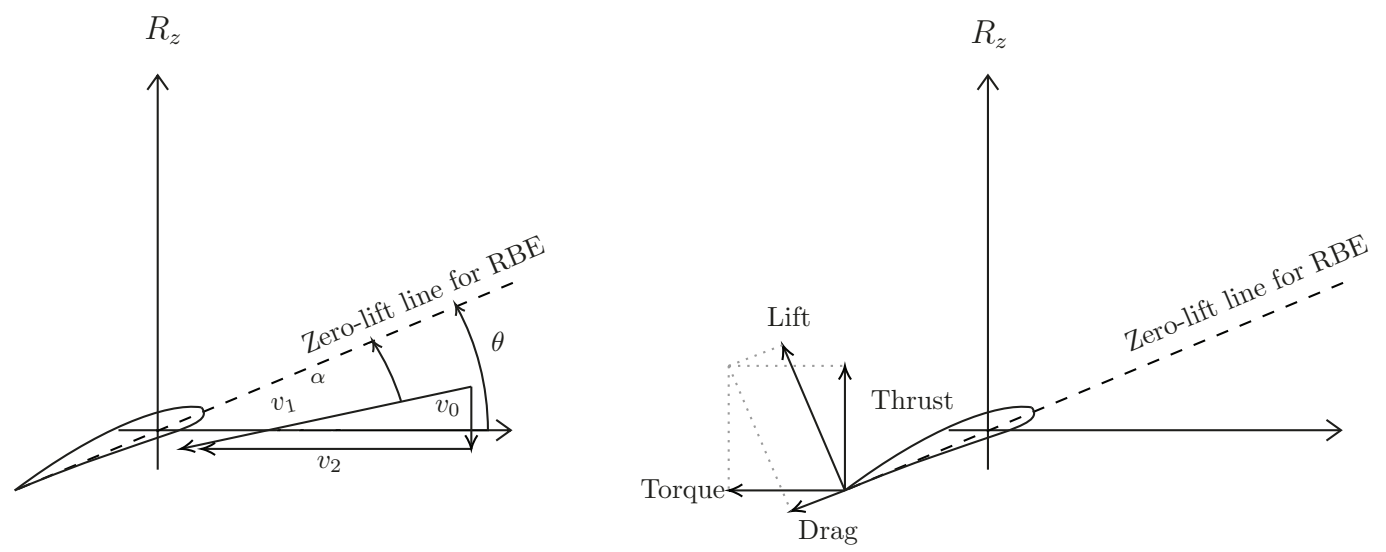

Figure 4.11: Rotor blade element airflow and forces diagram.

model, the wind velocity expressed in the inertial frame is

$$
{ }_{N}^{N} \overrightarrow{\boldsymbol{v}}^{W}=\left\{\begin{array}{c}
v_{\text {wind }, x} \\
v_{\text {wind,y }} \\
v_{\text {wind, }, z}
\end{array}\right\}
$$

Then the general expression of velocity for the $h$ th RBE at $l$ th azimuth $\psi_{l}$ with respect to the wind, and expressed in the inertial frame, is

$$
\begin{aligned}
{ }_{N}^{W} \overrightarrow{\boldsymbol{v}}^{e_{h} \psi_{l}}= & { }_{N}^{N} \overrightarrow{\boldsymbol{v}}^{e_{h} \psi_{l}}-{ }_{N}^{N} \overrightarrow{\boldsymbol{v}}^{W} \\
= & { }_{N}^{N} \dot{\overrightarrow{\boldsymbol{r}}}^{H}+\left[\boldsymbol{T}_{N \leftarrow H}\right]\left[\widetilde{N_{H}^{N} \overrightarrow{\boldsymbol{\omega}}^{H}}\right]_{H}^{H} \overrightarrow{\boldsymbol{r}}^{R} \\
& \left.+\left[\boldsymbol{T}_{N \leftarrow H}\right] \widetilde{{ }_{H}^{N} \overrightarrow{\boldsymbol{\omega}}^{H}}\right]\left[\boldsymbol{T}_{H \leftarrow R}\right]_{R}^{R} \overrightarrow{\boldsymbol{r}}^{e_{h} \psi_{l}} \\
& +\left[\boldsymbol{T}_{N \leftarrow R}\right]\left[\widetilde{H_{R}^{H} \overrightarrow{\boldsymbol{\omega}}^{R}}\right]_{R}^{R} \overrightarrow{\boldsymbol{r}}^{e_{h} \psi_{l}}-{ }_{N}^{N} \overrightarrow{\boldsymbol{v}}^{W}, \quad h=1, \ldots, N_{e}, \quad l=1, \ldots, N_{\psi}
\end{aligned}
$$

Equation 4.66 expressed in the rotor disc frame $R$ at $l$ th azimuth $\psi_{l}$ becomes

$$
\begin{aligned}
{ }_{R}^{W} \overrightarrow{\boldsymbol{v}}^{e_{h} \psi_{l}}= & {\left[\boldsymbol{T}_{R \leftarrow N}\right]_{N}^{W} \overrightarrow{\boldsymbol{v}}^{e_{h} \psi_{l}} } \\
= & {\left.\left[\boldsymbol{T}_{R \leftarrow N}\right]_{N}^{N} \dot{\overrightarrow{\boldsymbol{r}}}^{H}+\left[\boldsymbol{T}_{R \leftarrow H}\right] \widetilde{N_{H}^{N} \overrightarrow{\boldsymbol{\omega}}^{H}}\right]_{H}^{H} \overrightarrow{\boldsymbol{r}}^{R} } \\
& +\left[\boldsymbol{T}_{R \leftarrow H}\right]\left[\widetilde{\overrightarrow{\boldsymbol{\omega}}^{N}}\right]\left[\boldsymbol{T}_{H \leftarrow R}\right]_{R}^{R} \overrightarrow{\boldsymbol{r}}_{h} \psi_{l} \\
& +\left[\widetilde{{ }_{R}^{H} \overrightarrow{\boldsymbol{\omega}}^{R}}\right]_{R}^{R} \overrightarrow{\boldsymbol{r}}^{e_{h} \psi_{l}}-\left[\boldsymbol{T}_{R \leftarrow N}\right]_{N}^{N} \overrightarrow{\boldsymbol{v}}^{W}, \quad h=1, \ldots, N_{e}, \quad l=1, \ldots, N_{\psi}
\end{aligned}
$$

Now, the element flow velocities can be developed. First, the tangential flow $v_{2_{h l}}$ at the $h$ th $\mathrm{RBE}$ is then

$$
v_{2_{h l}}={ }_{R}^{W} \overrightarrow{\boldsymbol{v}}^{e_{h} \psi_{l}} \cdot\left(\frac{\hat{R}_{z} \times{ }_{R}^{R} \overrightarrow{\boldsymbol{r}}^{e_{h} \psi_{l}}}{\left\|\hat{R}_{z} \times{ }_{R}^{R} \overrightarrow{\boldsymbol{r}}^{e_{h} \psi_{l}}\right\|}\right), \quad h=1, \ldots, N_{e}, \quad l=1, \ldots, N_{\psi}
$$


The parenthetical expression in Equation 4.68 is the unit vector representing the direction of motion for the $h$ th RBE. Next, the axial flow $v_{0_{h l}}$ at the $h$ th RBE is

$$
v_{0_{h l}}=v_{i_{h l}}+v_{c_{h l}}, \quad h=1, \ldots, N_{e}, \quad l=1, \ldots, N_{\psi}
$$

where the climbing velocity $v_{c_{h l}}$ at the RBE is

$$
v_{c_{h l}}={ }_{R}^{W} \overrightarrow{\boldsymbol{v}}^{e_{h} \psi_{l}} \cdot \hat{R}_{z}, \quad h=1, \ldots, N_{e}, \quad l=1, \ldots, N_{\psi}
$$

and where the induced velocity $v_{i_{h l}}$ at the RBE is

$$
\begin{aligned}
v_{i_{h l}} & =v_{2_{h l}}\left[-(A)+\sqrt{(A)^{2}+B-C}\right] \quad h=1, \ldots, N_{e}, \quad l=1, \ldots, N_{\psi} \\
A & =\frac{\frac{\mathrm{d} C_{L}}{\mathrm{~d} \alpha_{h l}} c_{h}}{16 \pi R_{b}}+\frac{v_{c_{h l}}}{2 v_{2_{h l}}} \\
B & =\frac{\frac{\mathrm{d} C_{L} c_{h l}}{\mathrm{~d} c_{h}} \theta_{h} r}{8 \pi R_{b}^{2}} \\
C & =\frac{\frac{\mathrm{d} C_{L}}{\mathrm{~d} C_{h l}} c_{h} v_{c_{h l}}}{8 \pi R_{b} v_{2_{h l}}}
\end{aligned}
$$

The induced velocity is obtained from the solution of the quadratic formulation of equating element lift from basic lifting and momentum theories [43]. The element lift coefficient gradient $\frac{\mathrm{d} C_{L}}{\mathrm{~d} \alpha_{h l}}$ is corrected for Mach Number $M_{h l}$ in the $0 \leq M \leq 0.84$ region. The closed form correction is taken from Smith [53] as

$$
\begin{aligned}
\frac{\mathrm{d} C_{L}}{\mathrm{~d} \alpha_{h l}}= & \frac{\mathrm{d} C_{L}}{\mathrm{~d} \alpha_{h l}}\left(M=0, R e=10^{6}\right) \\
& \left\{\mu_{h l}+\frac{t / c_{h}}{1+t / c_{h}}\left[\mu_{h l}\left(\mu_{h l}-1\right)+\frac{1}{4}(\gamma+1)\left(\mu_{h l}^{2}-1\right)^{2}\right]\right\} \\
\mu_{h l}= & \left(\sqrt{1-M_{h l}^{2}}\right)^{-1} \\
\gamma= & 1.4
\end{aligned}
$$

Lastly, knowing the element axial and tangential flow velocities, the element flow velocity $v_{1_{h l}}$ is obtained by

$$
v_{1_{h l}}=\sqrt{v_{0_{h l}}^{2}+v_{2_{h l}}^{2}}
$$

In addition, the element angle of attack $\alpha_{h l}$ can be obtained from these velocities and the total blade pitch setting:

$$
\alpha_{h l}=\theta_{h l}-\phi_{h l}
$$


where

$$
\begin{aligned}
\phi_{h l} & =\tan ^{-1}\left(\frac{v_{0_{h l}}}{v_{2_{h l}}}\right) \\
\theta_{h l} & =\theta_{t_{h}}+\theta_{\text {coll }}+\theta_{c y c_{l}} \\
\theta_{c y c_{l}} & =\theta_{c y c, \text { max }} \cos \left(\psi_{l}-\psi_{\text {max }}\right)
\end{aligned}
$$

and where $\theta_{h l}$ is the element total blade pitch setting, $\phi_{h l}$ is the element flow angle relative to the rotor disc plane at $\operatorname{RBE} e_{h}$, and $\theta_{c y c, \max }$ is the maximum second harmonic cyclic pitch setting contribution at azimuth $\psi_{\max }$.

The development of Equations 4.65 through 4.79 lead to the elementary lift of an $\mathrm{RBE}$ acting at the rotor disc. The element force is transformed to an equipollent force and moment acting on the airframe in $H$ as

$$
\begin{aligned}
& { }_{R}^{R} \overrightarrow{\boldsymbol{F}}^{e_{h} \psi_{l}}=\left\{\begin{array}{c}
0 \\
0 \\
\frac{1}{2} \rho_{a i r} v_{1_{h l}}^{2} c_{h} \mathrm{~d} r\left[C_{L_{h l}} \cos (\phi)-C_{D_{h l}} \sin (\phi)\right]
\end{array}\right\} \\
& { }_{H}^{H} \overrightarrow{\boldsymbol{F}}^{e_{h} \psi_{l}}=\left[\boldsymbol{T}_{H \leftarrow R}\right]_{R}^{R} \overrightarrow{\boldsymbol{F}}^{e_{h} \psi_{l}} \\
& { }_{H}^{H} \overrightarrow{\boldsymbol{M}}^{e_{h} \psi_{l}}=\left[\widetilde{{ }_{H}^{H} \overrightarrow{\boldsymbol{r}}_{h} \psi_{l}}\right]\left[\boldsymbol{T}_{H \leftarrow R}\right]_{R}^{R} \overrightarrow{\boldsymbol{F}}^{e_{h} \psi_{l}}
\end{aligned}
$$

where

$$
\begin{aligned}
C_{L_{h l}} & =\frac{\mathrm{d} C_{L}}{\mathrm{~d} \alpha_{h l}} \alpha_{h l}+C_{L, \alpha_{0, h}} \\
C_{D_{h l}} & =\frac{\mathrm{d} C_{D}}{\mathrm{~d} \alpha_{h l}} \alpha_{h l}+C_{D, \alpha_{0, h}}
\end{aligned}
$$

where the lift coefficient $C_{L_{h}}$ and drag coefficient $C_{D_{h}}$ are expressed as linear functions proportional to the angle of attack, where $\frac{\mathrm{d} C_{L}}{\mathrm{~d} \alpha_{h l}}$ and $\frac{\mathrm{d} C_{D}}{\mathrm{~d} \alpha_{h l}}$ are the coefficient gradients corrected for Mach number, and $C_{D, \alpha_{0, h}}$ and $C_{L, \alpha_{0, h}}$ being the coefficient values at $\alpha_{h}=0$.

To remove the dependency on a propagated state-variable in calculating rotor disc thrust and moment, an azimuth-averaged approach is taken. The average force and moment generated by an RBE is taken through one full revolution around the rotor disc then summed over the length of the rotor blade because analytical formulation and integration of Equations 4.81 and 4.82 through one revolution is impractical. Hence the BEM mesh described in Figure 4.11. 
The average thrust of an $\mathrm{RBE} e_{h}$ at radius $r_{h}$ over one full rotor disc revolution $\psi=[0,2 \pi]$ is

$$
\begin{gathered}
{ }_{H}^{H} \overrightarrow{\boldsymbol{F}}_{\text {avg }}^{e_{h}}=\frac{1}{N_{\psi}} \sum_{l=1}^{N_{\psi}}{ }_{H}^{H} \overrightarrow{\boldsymbol{F}}^{e_{h} \psi_{l}} \quad h=1, \ldots, N_{e} \\
{ }_{H}^{H} \overrightarrow{\boldsymbol{M}}_{\text {avg }}^{e_{h}}=\frac{1}{N_{\psi}} \sum_{l=1}^{N_{\psi}}{ }_{H}^{H} \overrightarrow{\boldsymbol{M}}^{e_{h} \psi_{l}} \quad h=1, \ldots, N_{e}
\end{gathered}
$$

The average thrust and pitching moment is then summed over the effective length of the rotor blade, from the effective inner radius $r_{1}=R_{i} R_{b}$ to the effective outer radius $r_{N_{e}}=R_{o} R_{b}$, to determine the total thrust and pitching moment generated on the airframe by the complete BEM model:

$$
\begin{aligned}
{ }_{H}^{H} \overrightarrow{\boldsymbol{F}}^{B E M} & =N_{b} \sum_{h=1}^{N_{e}}{ }_{H}^{H} \overrightarrow{\boldsymbol{F}}^{e_{h}}{ }_{a v g} \\
{ }_{H}^{H} \overrightarrow{\boldsymbol{M}}^{B E M} & =N_{b} \sum_{h=1}^{N_{e}}{ }_{H}^{H} \overrightarrow{\boldsymbol{M}}^{e_{h}}{ }_{a v g}
\end{aligned}
$$

where $N_{b}$ is the number of rotor blades.

The aerodynamic models included in Section 4.3 have been developed in three dimensions. For the planar case of SRAMSS, the kinetic quantities expressed in Equations 4.63, 4.64, 4.88, and 4.89 are projected onto the two-dimensional plane of the simulation environment.

\subsection{Other Force-generating Elements}

Sections $4.1,4.2$, and 4.3 develop the kinetic quantities that act on either the rigidbody airframe, or the flexible-body skid landing gear. The dynamic finite element landing gear model, in conjunction with the contact dynamics model generate the primary forces acting on the landing gear. Whereas the aerodynamic drag and BEM models generate the primary forces acting on the aircraft's airframe.

Yet, there are forces which act on both sets of bodies. In Section 4.4.1 the expressions for the unknown forces at the interface between the rigid and flexible bodies will be developed. The known external damper forces acting between the rigid airframe and flexible landing gear are developed in Section 4.4.2. The gravitational forces are developed in Section 4.4.3. Finally the remaining inertial force terms are developed in Section 4.5. 


\subsubsection{Flexible-Rigid Body Interface Model}

Since the DFE landing gear model is developed separately from the rest of the aircraft, upon assembly into the final dynamic system, the internal loads at the DFE nodes interfacing to the rigid airframe must be considered as illustrated in Figure 4.12. More specifically, the forces acting on both must be equipollent since the landing gear and airframe are ultimately considered part of the same aircraft body. This represents the final consideration which must be made to combine rigid and flexible body dynamics in Kane's method

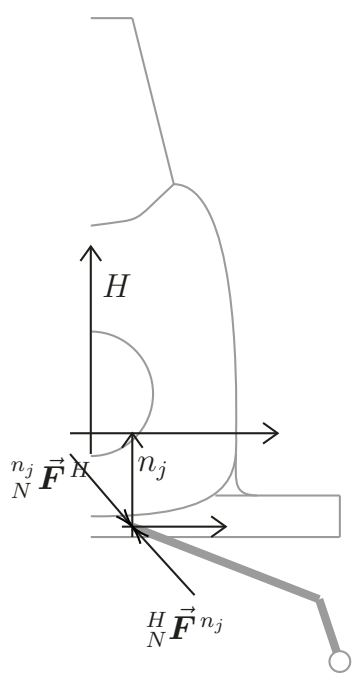

Figure 4.12: Interface of DFE nodes to the airframe.

Hence, the unknown nodal forces vector ${ }_{n}^{n} \boldsymbol{F}_{u}$ acting on the DFE nodes must also be considered to be acting on the airframe in $H$. Since the interface nodes are attached to the airframe, applying Newton's third law for interface DFE node indices $j_{u}$, must yield equal in magnitude but opposing interface forces. The forces and moments For rigid DFE node interfaces are

$$
\begin{aligned}
& { }_{H}^{H} \overrightarrow{\boldsymbol{F}}_{u}=-\sum_{j=j_{u}}\left[\boldsymbol{T}_{H \leftarrow n_{j}}\right]_{n_{j}}^{n_{j}} \overrightarrow{\boldsymbol{F}}_{u} \\
& \left.{ }_{H}^{H} \overrightarrow{\boldsymbol{M}}_{u}=-\sum_{j=j_{u}}\left(\widetilde{{ }_{H}^{H} \overrightarrow{\boldsymbol{r}}^{n_{j}}}\right]\left[\boldsymbol{T}_{H \leftarrow n_{j}}\right]_{n_{j}}^{n_{j}} \overrightarrow{\boldsymbol{F}}_{u}-\left[\boldsymbol{T}_{H \leftarrow n_{j}}\right]_{n_{j}}^{n_{j}} \overrightarrow{\boldsymbol{M}}_{u}\right)
\end{aligned}
$$

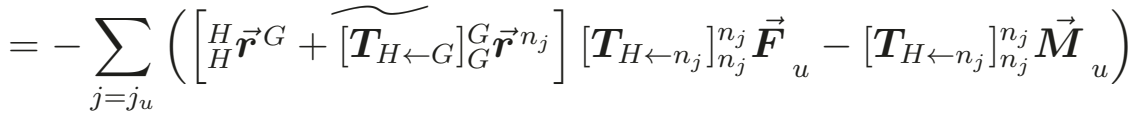

The unknown nodal forces are expressed in the nodal frames, therefore to agree with Newton's third law, the unknown forces acting on the airframe must be negative to 
satisfy $\sum \overrightarrow{\boldsymbol{F}}={ }_{n}^{H} \overrightarrow{\boldsymbol{F}}_{u}+{ }_{n_{j}}^{n_{j}} \overrightarrow{\boldsymbol{F}}_{u}=0$.

For hinged DFE node interfaces, where the interface node is free to rotate about the $z$ axis, the forces and moments are

$$
\begin{aligned}
& { }_{H}^{H} \overrightarrow{\boldsymbol{F}}_{u}=-\sum_{j=j_{u}}\left[\boldsymbol{T}_{H \leftarrow n_{j}}\right]_{n_{j}}^{n_{j}} \overrightarrow{\boldsymbol{F}}_{u} \\
& \left.{ }_{H}^{H} \overrightarrow{\boldsymbol{M}}_{u}=-\sum_{j=j_{u}}\left(\widetilde{{ }_{H}^{H} \overrightarrow{\boldsymbol{r}}^{n_{j}}}\right]\left[\boldsymbol{T}_{H \leftarrow n_{j}}\right]_{n_{j}}^{n_{j}} \overrightarrow{\boldsymbol{F}}_{u}\right)
\end{aligned}
$$

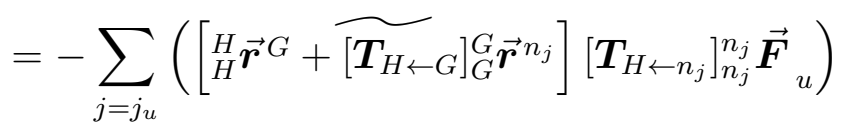

For the fixed degrees of freedom in each interface node, their generalized coordinates and speeds relative to the $G$ frame are prescribed.

\subsubsection{External Damper Model}

The structure of skid landing gear typically does not provide adequate damping for energy dissipation during contact. Often in helicopter design, this is addressed by the addition of discrete external dampers and hinged members in the skid structure configuration. Using the compression axis vector between the two mounting points of the damper, a damper force can be calculated as a directed force element then applied at both damper mount points.

For the generic aircraft used in the planar case of SRAMSS as shown in Figure 4.13, the external damper is mounted to the rigid airframe on one side, and at a DFE node on the other.

The directed force element in SRAMSS, used to model external dampers, is a simple spring-damper system for verification purposes. However, any suitable model can be used.

Given $N_{d}$ number of external dampers integrated into the aircraft, the relative position and speed along the $d$ th damper's compression axis must be determined. The position of the $d$ th airframe mount $M_{d}$ relative to the airframe's frame $H$, expressed in the $H$ frame, is ${ }_{H}^{H} \overrightarrow{\boldsymbol{r}}^{M_{d}}$. The $n_{j_{d}}$ th DFE node mount is

$$
{ }_{H}^{H} \overrightarrow{\boldsymbol{r}}^{n_{j}}={ }_{H}^{H} \overrightarrow{\boldsymbol{r}}^{G}+\left[\boldsymbol{T}_{H \leftarrow G}\right]_{G}^{G} \overrightarrow{\boldsymbol{r}}^{n_{j_{d}}}
$$

The position of the DFE node mount $n_{j_{d}}$ relative to the airframe mount $M_{d}$ becomes

$$
{ }_{H}^{M_{d}} \overrightarrow{\boldsymbol{r}}^{n_{j_{d}}}={ }_{H}^{H} \overrightarrow{\boldsymbol{r}}^{n_{j_{d}}}-{ }_{H}^{H} \overrightarrow{\boldsymbol{r}}^{M_{d}}
$$




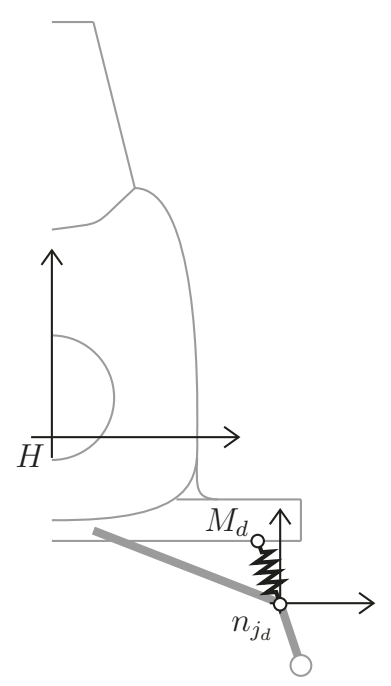

Figure 4.13: Discrete external damper mounting locations.

Differentiating Equation 4.93 yields the velocity of $n_{j_{d}}$ relative to the airframe mount $M_{d}$ :

$$
\begin{aligned}
& { }_{H}^{M_{d}} \overrightarrow{\boldsymbol{v}}^{n_{j_{d}}}=\frac{\mathrm{d}}{\mathrm{d} t}{ }^{M_{d}} \overrightarrow{\boldsymbol{r}}^{n_{j_{d}}} \\
& =\frac{\mathrm{d}}{\mathrm{d} t}\left({ }_{H}^{H} \overrightarrow{\boldsymbol{r}}^{n_{j}}\right)-\frac{\mathrm{d}}{\mathrm{d} t}\left({ }_{H}^{H} \overrightarrow{\boldsymbol{r}}^{M_{d}}\right) \\
& =\frac{\mathrm{d}}{\mathrm{d} t}\left({ }_{H}^{H} \overrightarrow{\boldsymbol{r}}^{G}+\left[\boldsymbol{T}_{H \leftarrow G}\right]_{G}^{G} \overrightarrow{\boldsymbol{r}}^{n_{j}}\right)-\frac{\mathrm{d}}{\mathrm{d} t}\left({ }_{H}^{H} \overrightarrow{\boldsymbol{r}}^{M_{d}}\right) \\
& ={ }_{H}^{H} \dot{\overrightarrow{\boldsymbol{r}}} G+\left[\dot{\boldsymbol{T}}_{H \leftarrow G}\right]_{G}^{G} \overrightarrow{\boldsymbol{r}}^{n_{j_{d}}}+\left[\boldsymbol{T}_{H \leftarrow G}\right]_{G}^{G} \dot{\overrightarrow{\boldsymbol{r}}} n_{j_{d}}-{ }_{H}^{H} \dot{\overrightarrow{\boldsymbol{r}}} M_{d}
\end{aligned}
$$

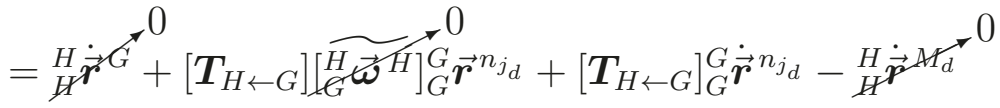

$$
\begin{aligned}
& =\left[\boldsymbol{T}_{H \leftarrow G}\right]_{G}^{G} \dot{\overrightarrow{\boldsymbol{r}}} n_{j_{d}}
\end{aligned}
$$

The unit vector expressing the orientation of the compression axis is required to obtain the relative position and velocity for the spring-damper model. The unit vector describing the orientation of $n_{j_{d}}$ with respect to $M_{d}$ is

$$
{ }_{H}^{M_{d}} \hat{\boldsymbol{r}}^{n_{j_{d}}}=\frac{{ }^{M_{d}} \overrightarrow{\boldsymbol{r}}^{n_{j_{d}}}}{\left\|\begin{array}{l}
M_{d} \\
{ }^{H}
\end{array} \overrightarrow{\boldsymbol{r}}^{n_{j_{d}}}\right\|}
$$

With Equation 4.95, the compressed damper length $\Delta_{d}$ and velocity $\dot{\Delta}_{d}$ along the $d$ th damper compression axis is

$$
\begin{aligned}
\Delta_{d} & ={ }_{H}^{M_{d}} \overrightarrow{\boldsymbol{r}}^{n_{j_{d}}} \cdot{ }_{H}^{M_{d}} \hat{\boldsymbol{r}}^{n_{j_{d}}} \\
\dot{\Delta}_{d} & ={ }_{H}^{M_{d}} \overrightarrow{\boldsymbol{v}}^{n_{j_{d}}} \cdot{ }_{H}^{M_{d}} \hat{\boldsymbol{r}}^{n_{j_{d}}}
\end{aligned}
$$


Then the damper force generated in the positive direction along the compression axis unit vector is

$$
F_{d_{d}}=-K_{d}\left(\Delta_{d}-\Delta_{d, 0}\right)-C_{d} \dot{\Delta}_{d}
$$

where $F_{d_{d}}$ is the force generated by the $d$ th external damper expressed along the compression axis ${ }_{H}^{M_{d}} \hat{\boldsymbol{r}}^{n_{j_{d}}}, K_{d}$ and $C_{d}$ are the stiffness and damping coefficients for the external damper spring-damper model, and $\Delta_{d, 0}$ is the uncompressed damper length.

Multiplied by the compression axis unit vector, the external damper forces acting on the airframe mount $M_{d}$ and on the DFE node $n_{j_{d}}$, expressed in the $H$ frame are

$$
\begin{aligned}
& { }_{H}^{M_{d}} \overrightarrow{\boldsymbol{F}}^{d_{d}}=-F_{d_{d} H}^{M_{d}} \hat{\boldsymbol{r}}^{n_{j_{d}}} \\
& { }_{H}^{n_{j_{d}}} \overrightarrow{\boldsymbol{F}}^{d_{d}}=F_{d_{d} H}{ }_{d} \hat{\boldsymbol{r}}^{n_{j_{d}}}
\end{aligned}
$$

Transforming Equations 4.100 to the nodal frame, assuming the force is acting directly at the node, yields:

$$
\begin{aligned}
n_{j_{d}} & \overrightarrow{\boldsymbol{F}}^{d_{d}} \\
n_{j_{d}} & =\left[\boldsymbol{T}_{n_{j_{d}} \leftarrow H}\right]_{H}^{n_{j_{d}}} \overrightarrow{\boldsymbol{F}}^{d_{d}} \\
{ }_{j_{j_{d}}} \overrightarrow{\boldsymbol{M}}^{d_{d}} & =0
\end{aligned}
$$

Assembly into the vector of known external nodal forces due to external dampers ${ }_{n}^{n} \boldsymbol{F}_{k}^{d}$ takes the form:

$$
{ }_{n}^{n} \boldsymbol{F}_{k}^{d}=\left\{\begin{array}{c}
{ }_{n_{1}} \overrightarrow{\boldsymbol{F}}^{d} \\
n_{1} \\
n_{1} \overrightarrow{\boldsymbol{M}}^{d} \\
{ }_{1} \\
\vdots \\
n_{j} \overrightarrow{\boldsymbol{F}}^{d} \\
n_{j} \\
n_{j} \\
n_{j} \overrightarrow{\boldsymbol{M}}^{d}
\end{array}\right\}
$$

where

$$
\left\{\begin{array}{c}
n_{j} \overrightarrow{\boldsymbol{F}}^{d} \\
n_{j_{j}} \\
n_{j} \overrightarrow{\boldsymbol{M}}^{d}
\end{array}\right\}=\left\{\begin{array}{c}
\sum^{d} \begin{array}{c}
n_{j} \\
n_{j}
\end{array} \\
0
\end{array}\right\}, \quad j=1, \ldots, N_{n} \forall j=j_{d}
$$

otherwise

$$
\left\{\begin{array}{c}
n_{n_{j}} \overrightarrow{\boldsymbol{F}}^{d} \\
n_{j} \\
n_{j} \\
n_{j}
\end{array} \overrightarrow{\boldsymbol{M}}^{d}\right\}=\left\{\begin{array}{l}
0 \\
0
\end{array}\right\}, \quad j=1, \ldots, N_{n} \forall j \neq j_{d}
$$

The external damper forces acting at the airframe mounts $M_{d}$ are transformed to 
equipollent forces and moments acting on the airframe

$$
\begin{aligned}
{ }_{H}^{H} \overrightarrow{\boldsymbol{F}}^{d} & =\sum_{d=1}^{N_{d}}{ }_{H}^{M_{d}} \overrightarrow{\boldsymbol{F}}^{d_{d}} \\
{ }_{H}^{H} \overrightarrow{\boldsymbol{M}}^{d} & =\sum_{d=1}^{N_{d}} \widetilde{\left[{ }_{H} \overrightarrow{\boldsymbol{r}}^{M_{d}}\right]_{H}^{M_{d}}} \overrightarrow{\boldsymbol{F}}^{d_{d}}
\end{aligned}
$$

\subsubsection{Gravitational Model}

The final active force required for the system is the gravitational force acting on the airframe and all DFE nodes. The gravitational forces on each body are

$$
\begin{aligned}
& { }_{H}^{H} \overrightarrow{\boldsymbol{F}}^{g}=m_{H}\left[\boldsymbol{T}_{H \leftarrow N}\right]\left\{\begin{array}{l}
0 \\
g
\end{array}\right\} \\
& { }_{n}^{n} \boldsymbol{F}_{k}^{g}=[\boldsymbol{M}]_{3 N_{n} \times 3 N_{n}}\left[\begin{array}{cc}
{\left[\boldsymbol{T}_{n_{1} \leftarrow N}\right]_{2 \times 2}} & {[0]_{2 \times 1}} \\
{[0]_{1 \times 2}} & 1 \\
\vdots & \vdots \\
{\left[\boldsymbol{T}_{n_{j} \leftarrow N}\right]_{2 \times 2}} & {[0]_{2 \times 1}} \\
{[0]_{1 \times 2}} & 1
\end{array}\right]_{3 N_{n} \times 3} \quad\left\{\begin{array}{c}
0 \\
g \\
0
\end{array}\right\} \text { for } j=1, \ldots, N_{n}
\end{aligned}
$$

where $m_{H}$ is the mass of the airframe, $[\boldsymbol{M}]$ is the total structure mass matrix of the DFE model, and $g=-9.81 \mathrm{~m} / \mathrm{s}$ is the gravitational acceleration constant. Since the gravitational acceleration vector acts in the intertial frame $N$, it must be transformed to the body frames before multiplication with the body masses.

\subsection{Inertial Forces}

At this point, Chapter 4 has developed the dynamic models required to generate the terms comprising the active forces $\overrightarrow{\boldsymbol{R}}^{k}$ and moments $\overrightarrow{\boldsymbol{T}}^{k}$, as well as inertial forces $\boldsymbol{R}^{* n}$ and moments $\boldsymbol{T}^{* n}$ acting at the DFE nodes. These are ready for introduction into Kane's equation. Only the inertial forces on the airframe remain to be developed. 
The translational and angular inertias of the airframe are

$$
\begin{aligned}
\overrightarrow{\boldsymbol{R}}^{* H} & =-m_{H H}^{N} \overrightarrow{\boldsymbol{a}}^{H} \\
& =-m_{H}\left(\left[{ }_{H}^{N} \boldsymbol{V}^{H}\right] \dot{\boldsymbol{u}}+\overrightarrow{\boldsymbol{Z}}_{H}\right) \\
\overrightarrow{\boldsymbol{T}}^{* H} & \left.=-I_{H}^{N} \overrightarrow{\boldsymbol{\alpha}}^{H}-\widetilde{{ }_{H}^{N} \overrightarrow{\boldsymbol{\omega}} H}\right]_{H H}^{N} \overrightarrow{\boldsymbol{\omega}}^{H} \\
& =-I_{H}\left(\left[{ }_{H}^{N} \boldsymbol{W}^{H}\right] \dot{\boldsymbol{u}}+\overrightarrow{\boldsymbol{Y}}_{H}\right)
\end{aligned}
$$

To re-iterate, the translational and angular inertias for all nodes, extracted from Equation 4.28, are

$$
\boldsymbol{R}^{* n}+\boldsymbol{T}^{* n}=-[\boldsymbol{M}]\left(\left[\boldsymbol{V}_{n}\right] \dot{\boldsymbol{u}}+\boldsymbol{Z}_{n}\right)
$$

This completes the kinetic development of the planar case of SRAMSS. The following section summarizes the active and inertial kinetic quantities.

\subsection{Summary of Kinetic Quantities}

The SRAMSS simulation environment comprises many dynamic models which act on various parts of the aircraft. Table 4.2 lists all inertial and active force terms acting on the airframe and DFE nodes. Each term or vector includes the associated equation number assigned during the developments in Chapter 4.

The structure of dynamic model interactions can be summarized as follows. First, the entire aircraft response is modelled by a mass-coupled rigid airframe and flexible dynamic finite element skid landing gear representation through a novel integration of DFE modelling in Kane's method. The two categories of bodies are mass coupled by the interface force model which is represented by an internal force compiled to the active force and moment term acting on the aircraft, and to the nodal forces vector acting at the DFE nodes. Second, rotor disc thrust, body drag forces, gravitational forces, and external damper forces are compiled into the active force and torque vectors acting on the rigid airframe. Third, The contact dynamics model which includes linear penalty normal forces and LuGre friction forces due to polygon contact, the DFE model gravitational forces, and external damper forces are compiled into the known external forces acting at the DFE nodes. The summation of the known external force and unknown interface forces constitute the total active forces and torques acting on the DFE node bodies. 
Table 4.2: Complete system forces summary

\begin{tabular}{|c|c|c|c|c|c|c|}
\hline \multirow[b]{2}{*}{ Forces } & \multicolumn{4}{|c|}{ Airframe } & \multicolumn{2}{|c|}{ DFE nodes } \\
\hline & $\overrightarrow{\boldsymbol{R}}^{* H}$ & $\overrightarrow{\boldsymbol{T}}^{* H}$ & $\overrightarrow{\boldsymbol{R}}^{H}$ & $\overrightarrow{\boldsymbol{T}}^{H}$ & $\boldsymbol{R}^{* n}+\boldsymbol{T}^{* n}$ & $\boldsymbol{R}^{n}+\boldsymbol{T}^{n}$ \\
\hline \multicolumn{7}{|l|}{ Inertial } \\
\hline Inertia & $\begin{array}{c}\overrightarrow{\boldsymbol{R}}^{* H} \\
(4.110)\end{array}$ & $\begin{array}{c}\overrightarrow{\boldsymbol{T}}^{* H} \\
(4.111)\end{array}$ & - & - & $\begin{array}{c}\boldsymbol{R}^{* n}+\boldsymbol{T}^{* n} \\
(4.112)\end{array}$ & - \\
\hline \multicolumn{7}{|l|}{ Active } \\
\hline Damping & - & - & - & - & - & $\begin{array}{c}-[\boldsymbol{C}]\left[\boldsymbol{V}_{n}\right] \boldsymbol{u} \\
(4.27)\end{array}$ \\
\hline Stiffness & - & - & - & - & - & $\begin{array}{c}-[\boldsymbol{K}] \boldsymbol{\delta}_{n} \\
(4.27)\end{array}$ \\
\hline Contact & - & - & - & - & - & $\begin{array}{c}{ }_{n}^{n} \boldsymbol{F}_{k}^{c} \\
(4.55)\end{array}$ \\
\hline Aero. drag & - & - & $\begin{array}{l}{ }_{H}^{H} \overrightarrow{\boldsymbol{F}}^{W} \\
(4.63)\end{array}$ & $\begin{array}{c}{ }_{H}^{H} \overrightarrow{\boldsymbol{M}}^{W} \\
\quad(4.64)\end{array}$ & - & - \\
\hline Rotor Disc & - & - & $\begin{array}{c}{ }_{H}^{H} \overrightarrow{\boldsymbol{F}}^{B E M} \\
\quad(4.88)\end{array}$ & $\begin{array}{c}{ }_{H}^{H} \overrightarrow{\boldsymbol{M}}^{B E M} \\
\quad(4.89)\end{array}$ & - & - \\
\hline Damper & - & - & $\begin{array}{c}{ }_{H}^{H} \overrightarrow{\boldsymbol{F}}^{d} \\
(4.106)\end{array}$ & $\begin{array}{c}{ }_{H}^{H} \overrightarrow{\boldsymbol{M}}^{d} \\
(4.107)\end{array}$ & - & $\begin{array}{c}{ }_{n}^{n} \boldsymbol{F}_{k}^{d} \\
(4.103)\end{array}$ \\
\hline Gravity & - & - & $\begin{array}{c}{ }_{H}^{H} \overrightarrow{\boldsymbol{F}}^{g} \\
(4.108)\end{array}$ & - & - & $\begin{array}{c}{ }_{n}^{n} \boldsymbol{F}_{k}^{g} \\
(4.109)\end{array}$ \\
\hline Interface & - & - & $\begin{array}{l}{ }_{H}^{H} \overrightarrow{\boldsymbol{F}}_{u} \\
(4.90)\end{array}$ & $\begin{array}{l}{ }_{H}^{H} \overrightarrow{\boldsymbol{M}}_{u} \\
(4.91)\end{array}$ & - & $\begin{array}{c}{ }_{n}^{n} \boldsymbol{F}_{u} \\
(4.30)\end{array}$ \\
\hline
\end{tabular}




\section{Chapter 5}

\section{System Assembly and Simulation Architecture}

Chapters 3 and 4 presented the development of the kinematic and kinetic terms required to develop the dynamical system according to Kane's method. This chapter will present the assembly of the developed terms into a single dynamical system, and manipulation of the system into a form suitable for numerical solutions and state propagation.

Section 5.1 develops the assembly of the system and its manipulation into a suitable form. Then, Section 5.2 covers the simulation architecture used in SRAMSS, with an emphasis on how the states are propagated, how contact events are handled, and the general file structure of SRAMSS.

\subsection{System Assembly}

As presented in Section 2.2, Kanes's method for the formulation of the governing dynamical equations is stated

$$
\boldsymbol{F}+\boldsymbol{F}^{*}=0
$$

with

$$
\begin{aligned}
\boldsymbol{F} & =\sum_{k=1}^{N_{B}}\left(\left[{ }_{k}^{N} V^{k}\right]^{T} \overrightarrow{\boldsymbol{R}}^{k}+\left[{ }_{k}^{N} W^{k}\right]^{T} \overrightarrow{\boldsymbol{T}}^{k}\right) \\
\boldsymbol{F}^{*} & =\sum_{k=1}^{N_{B}}\left(\left[{ }_{k}^{N} V^{k}\right]^{T} \overrightarrow{\boldsymbol{R}}^{* k}+\left[{ }_{k}^{N} W^{k}\right]^{T} \overrightarrow{\boldsymbol{T}}^{* k}\right)
\end{aligned}
$$


where $N_{B}$ is the number of bodies in the system. While the aircraft and its landing gear are considered a single physical body, the developments in Chapters 3 and 4 consider them to be individual flexible and rigid bodies. Equations 4.30 and 4.90 serve to unify the separate bodies into a single body with rigid and flexible characteristics. Separation into these smaller 'sub-bodies' allows the landing gear DFE model to be developed separately, and eases the integration of infinitely configurable landing gear into the governing equations.

The generic aircraft simulated in SRAMSS comprises one rigid body centred at the $H$ frame representing the airframe, and $N_{n}$ number of equivalent nodal bodies from the DFE model centred respectively at the $n_{j}$ frames representing the skid landing gear.

For the airframe body in $H$, and with the forces summary in Table 4.2 in Section 4.6, Equations 2.13 and 2.14 become

$$
\begin{aligned}
& \boldsymbol{F}^{H}=\left[{ }_{H}^{N} \boldsymbol{V}^{H}\right]^{T} \overrightarrow{\boldsymbol{R}}^{H}+\left[{ }_{H}^{N} \boldsymbol{W}^{H}\right]^{T} \overrightarrow{\boldsymbol{T}}^{H} \\
& =\left[{ }_{H}^{N} \boldsymbol{V}^{H}\right]^{T}\left({ }_{H}^{H} \overrightarrow{\boldsymbol{F}}^{W}+{ }_{H}^{H} \overrightarrow{\boldsymbol{F}}^{R B E}+{ }_{H}^{H} \overrightarrow{\boldsymbol{F}}^{d}+{ }_{H}^{H} \overrightarrow{\boldsymbol{F}}^{g}+{ }_{H}^{H} \overrightarrow{\boldsymbol{F}}_{u}\right) \\
& +\left[{ }_{H}^{N} \boldsymbol{W}^{H}\right]^{T}\left({ }_{H}^{H} \overrightarrow{\boldsymbol{M}}^{W}+{ }_{H}^{H} \overrightarrow{\boldsymbol{M}}^{R B E}+{ }_{H}^{H} \overrightarrow{\boldsymbol{M}}^{d}+{ }_{H}^{H} \overrightarrow{\boldsymbol{M}}_{u}\right) \\
& =\left[{ }_{H}^{N} \boldsymbol{V}^{H}\right]^{T}\left({ }_{H}^{H} \overrightarrow{\boldsymbol{F}}^{W}+{ }_{H}^{H} \overrightarrow{\boldsymbol{F}}^{R B E}+{ }_{H}^{H} \overrightarrow{\boldsymbol{F}}^{d}+{ }_{H}^{H} \overrightarrow{\boldsymbol{F}}^{g}\right) \\
& +\left[{ }_{H}^{N} \boldsymbol{W}^{H}\right]^{T}\left({ }_{H}^{H} \overrightarrow{\boldsymbol{M}}^{W}+{ }_{H}^{H} \overrightarrow{\boldsymbol{M}}^{R B E}+{ }_{H}^{H} \overrightarrow{\boldsymbol{M}}^{d}\right) \\
& -\left[\left[{ }_{H}^{N} \boldsymbol{V}^{H}\right]^{T}\left[{ }_{H}^{N} \boldsymbol{W}^{H}\right]^{T}\right]\left(\left[\boldsymbol{\tau}_{H \leftarrow n_{u}}\right]_{n}^{n} \boldsymbol{F}_{u}\right)
\end{aligned}
$$

and

$$
\begin{aligned}
\boldsymbol{F}^{* H}= & {\left[{ }_{H}^{N} \boldsymbol{V}^{H}\right]^{T} \overrightarrow{\boldsymbol{R}}^{* H}+\left[{ }_{H}^{N} \boldsymbol{W}^{H}\right]^{T} \overrightarrow{\boldsymbol{T}}^{* H} } \\
= & -\left[{ }_{H}^{N} \boldsymbol{V}^{H}\right]^{T}\left(m_{H}{ }_{H}^{N} \overrightarrow{\boldsymbol{a}}^{H}\right)-\left[{ }_{H}^{N} \boldsymbol{W}^{H}\right]^{T}\left(I_{H}{ }_{H}^{N} \overrightarrow{\boldsymbol{\alpha}}^{H}\right) \\
= & -\left[{ }_{H}^{N} \boldsymbol{V}^{H}\right]^{T}\left(m_{H}\left(\left[{ }_{H}^{N} \boldsymbol{V}^{H}\right] \dot{\boldsymbol{u}}+\overrightarrow{\boldsymbol{Z}}_{H}\right)\right) \\
& -\left[{ }_{H}^{N} \boldsymbol{W}^{H}\right]^{T}\left(I_{H}\left(\left[{ }_{H}^{N} \boldsymbol{W}^{H}\right] \dot{\boldsymbol{u}}+\overrightarrow{\boldsymbol{Y}}_{H}\right)\right) \\
= & -\left[{ }_{H}^{N} \boldsymbol{V}^{H}\right]^{T} m_{H}\left[{ }_{H}^{N} \boldsymbol{V}^{H}\right] \dot{\boldsymbol{u}}-\left[{ }_{H}^{N} \boldsymbol{V}^{H}\right]^{T} m_{H} \overrightarrow{\boldsymbol{Z}}_{H} \\
& -\left[{ }_{H}^{N} \boldsymbol{W}^{H}\right]^{T} I_{H}\left[{ }_{H}^{N} \boldsymbol{W}^{H}\right] \dot{\boldsymbol{u}}-\left[{ }_{H}^{N} \boldsymbol{W}^{H}\right]^{T} I_{H} \overrightarrow{\boldsymbol{Y}}_{H}
\end{aligned}
$$

where $\left[\boldsymbol{\tau}_{H \leftarrow n_{u}}\right]$ is the matrix representation of all coefficients to each ${ }_{n_{j}}^{n_{j}} \overrightarrow{\boldsymbol{F}}_{u}$ in Equations 4.90 and 4.91, expressed

$$
\begin{aligned}
{\left[\boldsymbol{\tau}_{H \leftarrow n_{u}}\right]=} & {\left[\begin{array}{ccccc}
{\left[\boldsymbol{T}_{H \leftarrow n_{1}}\right]} & {[0]_{2 \times 1}} & \ldots & {\left[\boldsymbol{T}_{H \leftarrow n_{j}}\right]} & {[0]_{2 \times 1}} \\
{\left[\widehat{{ }_{H}^{H} \boldsymbol{\boldsymbol { r }}^{n_{1}}}\right]} & 1 & \ldots & {\left[\widehat{{ }_{H}^{H} \boldsymbol{\boldsymbol { r }}_{j}}\right]} & 1
\end{array}\right], } \\
& j=1, \ldots, N_{n} \forall j=j_{u}
\end{aligned}
$$


for rigidly fixed nodes, or

$$
\begin{gathered}
{\left[\boldsymbol{\tau}_{H \leftarrow n_{u}}\right]=\left[\begin{array}{ccc}
{\left[\boldsymbol{T}_{H \leftarrow n_{1}}\right]} & \ldots & {\left[\boldsymbol{T}_{H \leftarrow n_{j}}\right]} \\
{[0]_{2 \times 1}} & \ldots & {[0]_{2 \times 1}}
\end{array}\right],} \\
j=1, \ldots, N_{n} \forall j=j_{u}
\end{gathered}
$$

for hinged interface nodes. The matrix $\left[\boldsymbol{\tau}_{H \leftarrow n_{u}}\right]$ transforms the unknown nodal forces acting on the interface DFE nodes into equivalent but opposed forces ${ }_{H}^{H} \overrightarrow{\boldsymbol{F}}_{u}$ and moments ${ }_{H}^{H} \overrightarrow{\boldsymbol{M}}_{u}$ acting on the airframe as shown in the development of Equation 5.1.

In Equation 5.1, the unknown interface forces acting on the helicopter are expressed in terms of the unknown external nodal forces ${ }_{n}^{n} \boldsymbol{F}_{u}$ such that it can be isolated in the manipulation of the final dynamical system.

For the DFE node bodies in frames $n_{j}$, since the terms in Table 4.2 are vectors of nodal forces acting on all nodes, in all degrees of freedom, Equations 2.13 and 2.14 are written compactly as

$$
\begin{aligned}
\boldsymbol{F}^{n} & =\left[\boldsymbol{V}_{n}\right]^{T}\left(\boldsymbol{R}^{n}+\boldsymbol{T}^{n}\right) \\
& =\left[\boldsymbol{V}_{n}\right]^{T}\left({ }_{n}^{n} \boldsymbol{F}_{k}+{ }_{n}^{n} \boldsymbol{F}_{u}-[\boldsymbol{C}]\left[\boldsymbol{V}_{n}\right] \boldsymbol{u}-[\boldsymbol{K}] \boldsymbol{\delta}_{n}\right) \\
& =\left[\boldsymbol{V}_{n}\right]^{T}\left({ }_{n}^{n} \boldsymbol{F}_{k}-[\boldsymbol{C}]\left[\boldsymbol{V}_{n}\right] \boldsymbol{u}-[\boldsymbol{K}] \boldsymbol{\delta}_{n}\right)+\left[\boldsymbol{V}_{u}\right]_{n}^{T}{ }_{n}^{n} \boldsymbol{F}_{u}
\end{aligned}
$$

and

$$
\begin{aligned}
\boldsymbol{F}^{* n} & =\left[\boldsymbol{V}_{n}\right]^{T}\left(\boldsymbol{R}^{* n}+\boldsymbol{T}^{* n}\right) \\
& =-\left[\boldsymbol{V}_{n}\right]^{T}\left([\boldsymbol{M}]\left(\left[\boldsymbol{V}_{n}\right] \dot{\boldsymbol{u}}+\boldsymbol{Z}_{n}\right)\right) \\
& =-\left[\boldsymbol{V}_{n}\right]^{T}[\boldsymbol{M}]\left[\boldsymbol{V}_{n}\right] \dot{\boldsymbol{u}}-\left[\boldsymbol{V}_{n}\right]^{T}[\boldsymbol{M}] \boldsymbol{Z}_{n}
\end{aligned}
$$

For the system at hand, by Equation 2.13, the total active forces are the summation of Equations 5.1 and 5.4, such that in incorporates the airframe body and DFE node 
bodies as

$$
\begin{aligned}
& \boldsymbol{F}=\boldsymbol{F}^{H}+\boldsymbol{F}^{n} \\
& =\left[{ }_{H}^{N} \boldsymbol{V}^{H}\right]^{T}\left({ }_{H}^{H} \overrightarrow{\boldsymbol{F}}^{W}+{ }_{H}^{H} \overrightarrow{\boldsymbol{F}}^{R B E}+{ }_{H}^{H} \overrightarrow{\boldsymbol{F}}^{d}+{ }_{H}^{H} \overrightarrow{\boldsymbol{F}}^{g}\right) \\
& +\left[{ }_{H}^{N} \boldsymbol{W}^{H}\right]^{T}\left({ }_{H}^{H} \overrightarrow{\boldsymbol{M}}^{W}+{ }_{H}^{H} \overrightarrow{\boldsymbol{M}}^{R B E}+{ }_{H}^{H} \overrightarrow{\boldsymbol{M}}^{d}\right) \\
& -\left[\left[{ }_{H}^{N} \boldsymbol{V}^{H}\right]^{T}\left[{ }_{H}^{N} \boldsymbol{W}^{H}\right]^{T}\right]\left(\left[\boldsymbol{\tau}_{H \leftarrow n_{u}}\right]_{n}^{n} \boldsymbol{F}_{u}\right) \\
& +\left[\boldsymbol{V}_{n}\right]^{T}\left({ }_{n}^{n} \boldsymbol{F}_{k}-[\boldsymbol{C}]\left[\boldsymbol{V}_{n}\right] \boldsymbol{u}-[\boldsymbol{K}] \boldsymbol{\delta}_{n}\right)+\left[\boldsymbol{V}_{u}\right]^{T}{ }_{n}^{n} \boldsymbol{F}_{u} \\
& =\left[{ }_{H}^{N} \boldsymbol{V}^{H}\right]^{T}\left({ }_{H}^{H} \overrightarrow{\boldsymbol{F}}^{W}+{ }_{H}^{H} \overrightarrow{\boldsymbol{F}}^{R B E}+{ }_{H}^{H} \overrightarrow{\boldsymbol{F}}^{d}+{ }_{H}^{H} \overrightarrow{\boldsymbol{F}}^{g}\right) \\
& +\left[{ }_{H}^{N} \boldsymbol{W}^{H}\right]^{T}\left({ }_{H}^{H} \overrightarrow{\boldsymbol{M}}^{W}+{ }_{H}^{H} \overrightarrow{\boldsymbol{M}}^{R B E}+{ }_{H}^{H} \overrightarrow{\boldsymbol{M}}^{d}\right) \\
& +\left[\boldsymbol{V}_{n}\right]^{T}\left({ }_{n}^{n} \boldsymbol{F}_{k}-[\boldsymbol{C}]\left[\boldsymbol{V}_{n}\right] \boldsymbol{u}-[\boldsymbol{K}] \boldsymbol{\delta}_{n}\right) \\
& +\left(\left[\boldsymbol{V}_{u}\right]^{T}-\left[\left[{ }_{H}^{N} \boldsymbol{V}^{H}\right]^{T}\left[{ }_{H}^{N} \boldsymbol{W}^{H}\right]^{T}\right]\left[\boldsymbol{\tau}_{H \leftarrow n_{u}}\right]\right){ }_{n}^{n} \boldsymbol{F}_{u} \\
& \boldsymbol{F}=\boldsymbol{F}_{k}+[\boldsymbol{D}] \boldsymbol{F}_{u}
\end{aligned}
$$

and the total inertial forces as the summation of Equations 5.2 and 5.5:

$$
\begin{aligned}
\boldsymbol{F}^{*}= & \boldsymbol{F}^{* H}+\boldsymbol{F}^{* n} \\
= & -\left[{ }_{H}^{N} \boldsymbol{V}^{H}\right]^{T} m_{H}\left[{ }_{H}^{N} \boldsymbol{V}^{H}\right] \dot{\boldsymbol{u}}-\left[{ }_{H}^{N} \boldsymbol{V}^{H}\right]^{T} m_{H} \overrightarrow{\boldsymbol{Z}}_{H} \\
& -\left[{ }_{H}^{N} \boldsymbol{W}^{H}\right]^{T} I_{H}\left[{ }_{H}^{N} \boldsymbol{W}^{H}\right] \dot{\boldsymbol{u}}-\left[{ }_{H}^{N} \boldsymbol{W}^{H}\right]^{T} I_{H} \overrightarrow{\boldsymbol{Y}}_{H} \\
& -\left[{ }_{\boldsymbol{V}_{n}}\right]^{T}[\boldsymbol{M}]\left[\boldsymbol{V}_{n}\right] \dot{\boldsymbol{u}}-\left[\boldsymbol{V}_{n}\right]^{T}[\boldsymbol{M}] \boldsymbol{Z}_{n}
\end{aligned}
$$

Equation 5.7 can be rearranged to isolate the generalized accelerations vector $\dot{\boldsymbol{u}}$,

$$
\boldsymbol{F}^{*}=[\boldsymbol{A}] \dot{\boldsymbol{u}}+\boldsymbol{B}
$$

such that

$$
\begin{aligned}
\boldsymbol{F}^{*}= & -\left(m_{H}\left[{ }_{H}^{N} \boldsymbol{V}^{H}\right]^{T}\left[{ }_{H}^{N} \boldsymbol{V}^{H}\right]+\left[{ }_{H}^{N} \boldsymbol{W}^{H}\right]^{T} I_{H}\left[{ }_{H}^{N} \boldsymbol{W}^{H}\right]+\left[V_{n}\right]^{T}[M]\left[\boldsymbol{V}_{n}\right]\right) \dot{\boldsymbol{u}} \\
& -m_{H}\left[{ }_{H}^{N} \boldsymbol{V}^{H}\right]^{T} \overrightarrow{\boldsymbol{Z}}_{H}-I_{H}\left[{ }_{H}^{N} \boldsymbol{W}^{H}\right]^{T} \overrightarrow{\boldsymbol{Y}}_{H}-\left[\boldsymbol{V}_{n}\right]^{T}[\boldsymbol{M}] \boldsymbol{Z}_{n}
\end{aligned}
$$


Now, Equations 5.9 and 5.7 can be assembled as Equation 2.11, then solved for $\dot{\boldsymbol{u}}$.

$$
\begin{aligned}
& \boldsymbol{F}+\boldsymbol{F}^{*}=0 \\
& \boldsymbol{F}+[\boldsymbol{A}] \dot{\boldsymbol{u}}+\boldsymbol{B}=0 \\
& {[\boldsymbol{A}] \dot{\boldsymbol{u}} }=-\boldsymbol{B}-\boldsymbol{F} \\
& {[\boldsymbol{A}] \dot{\boldsymbol{u}} }=-\boldsymbol{B}-\boldsymbol{F}_{k}-[\boldsymbol{D}] \boldsymbol{F}_{u} \\
& {[\boldsymbol{A}] \dot{\boldsymbol{u}}=-\boldsymbol{C}-[\boldsymbol{D}] \boldsymbol{F}_{u} } \\
& {[\boldsymbol{A}]\left\{\begin{array}{l}
\dot{\boldsymbol{u}}_{P} \\
\dot{\boldsymbol{u}}_{H} \\
\dot{\boldsymbol{u}}_{G} \\
\dot{\boldsymbol{u}}_{n}
\end{array}\right\}=-\boldsymbol{C}-[\boldsymbol{D}] \boldsymbol{F}_{u} }
\end{aligned}
$$

with the respective partitions

$$
\begin{gathered}
{[\boldsymbol{A}]=\left[\begin{array}{cccc}
{\left[\boldsymbol{A}_{11}\right]_{12 \times 12}} & {\left[\boldsymbol{A}_{12}\right]_{12 \times 3}} & {\left[\boldsymbol{A}_{13}\right]_{12 \times 3}} & {\left[\boldsymbol{A}_{14}\right]_{12 \times 3 N_{n}}} \\
{\left[\boldsymbol{A}_{21}\right]_{3 \times 12}} & {\left[\boldsymbol{A}_{22}\right]_{3 \times 3}} & {\left[\boldsymbol{A}_{23}\right]_{3 \times 3}} & {\left[\boldsymbol{A}_{24}\right]_{3 \times 3 N_{n}}} \\
{\left[\boldsymbol{A}_{31}\right]_{3 \times 12}} & {\left[\boldsymbol{A}_{32}\right]_{3 \times 3}} & {\left[\boldsymbol{A}_{33}\right]_{3 \times 3}} & {\left[\boldsymbol{A}_{34}\right]_{3 \times 3 N_{n}}} \\
{\left[\boldsymbol{A}_{41}\right]_{3 N_{n} \times 12}} & {\left[\boldsymbol{A}_{42}\right]_{3 N_{n} \times 3}} & {\left[\boldsymbol{A}_{43}\right]_{3 N_{n} \times 3}} & {\left[\boldsymbol{A}_{44}\right]_{3 N_{n} \times 3 N_{n}}}
\end{array}\right]} \\
\dot{\boldsymbol{u}}=\left\{\begin{array}{c}
\left\{\dot{\boldsymbol{u}}_{P}\right\}_{12 \times 1} \\
\left\{\dot{\boldsymbol{u}}_{H}\right\}_{3 \times 1} \\
\left\{\dot{\boldsymbol{u}}_{G}\right\}_{3 \times 1} \\
\left\{\dot{\boldsymbol{u}}_{n}\right\}_{3 N_{n} \times 1}
\end{array}\right\} \\
\boldsymbol{C}=\left\{\begin{array}{c}
\left\{\boldsymbol{C}_{1}\right\}_{12 \times 1} \\
\left\{\boldsymbol{C}_{2}\right\}_{3 \times 1} \\
\left\{\boldsymbol{C}_{3}\right\}_{3 \times 1} \\
\left\{\boldsymbol{C}_{4}\right\}_{3 n_{n} \times 1}
\end{array}\right\} \\
\boldsymbol{F}_{u}=\left\{\boldsymbol{F}_{u}\right\}_{3 N_{u} \times 1} \\
{\left[\begin{array}{l}
{\left[\boldsymbol{D}_{1}\right]_{12 \times 3 N_{u}}} \\
{\left[\boldsymbol{D}_{2}\right]_{3 \times 3 N_{u}}} \\
{\left[\boldsymbol{D}_{3}\right]_{3 \times 3 N_{u}}} \\
{\left[\boldsymbol{D}_{4}\right]_{3 \times 3 N_{u}}}
\end{array}\right]}
\end{gathered}
$$

where $N_{u}$ is the number of DFE nodes interfacing with the airframe. Since $\dot{\boldsymbol{u}}_{P}$ is 
prescribed, and $\dot{\boldsymbol{u}}_{G}=0$, the system presented in Equation 5.10 reduces to

$$
\begin{aligned}
{\left[\begin{array}{ll}
{\left[\boldsymbol{A}_{22}\right]} & {\left[\boldsymbol{A}_{24}\right]} \\
{\left[\boldsymbol{A}_{42}\right]} & {\left[\boldsymbol{A}_{44}\right]}
\end{array}\right]\left\{\begin{array}{l}
\dot{\boldsymbol{u}}_{H} \\
\dot{\boldsymbol{u}}_{n}
\end{array}\right\}=-\left\{\begin{array}{l}
\boldsymbol{C}_{2} \\
\boldsymbol{C}_{4}
\end{array}\right\}-\left[\begin{array}{l}
{\left[\boldsymbol{D}_{2}\right]} \\
{\left[\boldsymbol{D}_{4}\right]}
\end{array}\right] \boldsymbol{F}_{u}-\left[\begin{array}{l}
{\left[\boldsymbol{A}_{21}\right]} \\
{\left[\boldsymbol{A}_{41}\right]}
\end{array}\right]\left\{\dot{\boldsymbol{u}}_{P o l y}\right\} } \\
{\left[\boldsymbol{A}^{\prime}\right]\left\{\begin{array}{l}
\dot{\boldsymbol{u}}_{H} \\
\dot{\boldsymbol{u}}_{n}
\end{array}\right\}=-\boldsymbol{C}^{\prime}-\left[\boldsymbol{D}^{\prime}\right] \boldsymbol{F}_{u} }
\end{aligned}
$$

For this work, the last term of Equation 5.16 can be eliminated since the contributions of the ship, RSD deck, and wedge clamp motions are accounted for through the contact forces since the polygons are not part of the aircraft's chain of frames.

The system in Equation 5.16 includes $\dot{\boldsymbol{u}}$ which contains known interface node accelerations $\dot{\boldsymbol{u}}_{n_{j}}=0, \forall j=j_{u}$. The vector of known nodal accelerations is equivalent in size to $\boldsymbol{F}_{u}$, therefore Equation 5.16 can be manipulated to express the solution vector containing the unknown generalized accelerations and unknown interface forces such that

$$
\begin{aligned}
& {\left[\begin{array}{llll}
{\left[\boldsymbol{A}_{H}^{\prime}\right]} & {\left[\boldsymbol{A}_{1}^{\prime}\right]} & \ldots & {\left[\boldsymbol{A}_{j}^{\prime}\right.}
\end{array}\right]\left\{\begin{array}{l}
\dot{\boldsymbol{u}}_{H} \\
\dot{\boldsymbol{u}}_{n}
\end{array}\right\}=-\boldsymbol{C}^{\prime}-\left[\boldsymbol{D}^{\prime}\right] \boldsymbol{F}_{u}, \quad j=1, \ldots, N_{n}} \\
& {\left[\begin{array}{ll}
{\left[\boldsymbol{A}^{\prime \prime}\right]} & {\left[\boldsymbol{D}^{\prime}\right.}
\end{array}\right]\left\{\begin{array}{l}
\dot{\boldsymbol{u}}_{H} \\
\dot{\boldsymbol{u}}_{n}^{\prime} \\
\boldsymbol{F}_{u}
\end{array}\right\}=-\boldsymbol{C}^{\prime}-\left[\overline{\boldsymbol{A}^{\prime \prime}}\right]\left\{\overline{\dot{\boldsymbol{u}}_{n}^{\prime}}\right\}^{0}} \\
& {\left[\boldsymbol{A}^{\prime \prime \prime}\right]\left\{\begin{array}{c}
\dot{\boldsymbol{u}}_{H} \\
\dot{\boldsymbol{u}}_{n}^{\prime} \\
\boldsymbol{F}_{u}
\end{array}\right\}=-\boldsymbol{C}^{\prime}}
\end{aligned}
$$

where: $\quad\left[\boldsymbol{A}^{\prime \prime}\right]=\left[\boldsymbol{A}_{a}^{\prime}\right] \forall a \neq j_{u}$

$$
\dot{\boldsymbol{u}}_{n}^{\prime}=\dot{\boldsymbol{u}}_{n_{j}} \forall j \neq j_{u}
$$

Equation 5.17 is the final linear system of equations in the form $\left[\boldsymbol{M}_{\text {sys }}\right] \boldsymbol{x}_{\text {sys }}=\boldsymbol{F}_{\text {sys }}$, and can be solved using numerical linear equation solvers. The size of the system to be solved is dependent on the number of unknown generalized accelerations and the size of the unknown interface forces vector. Once the solution $\boldsymbol{x}_{\text {sys }}$ is obtained, the interface force elements are removed from the system solution vector. The nonprescribed generalized coordinate time-derivatives are included for state-propagation 
purposes, such that the state derivative vector $\dot{\boldsymbol{x}}$ becomes

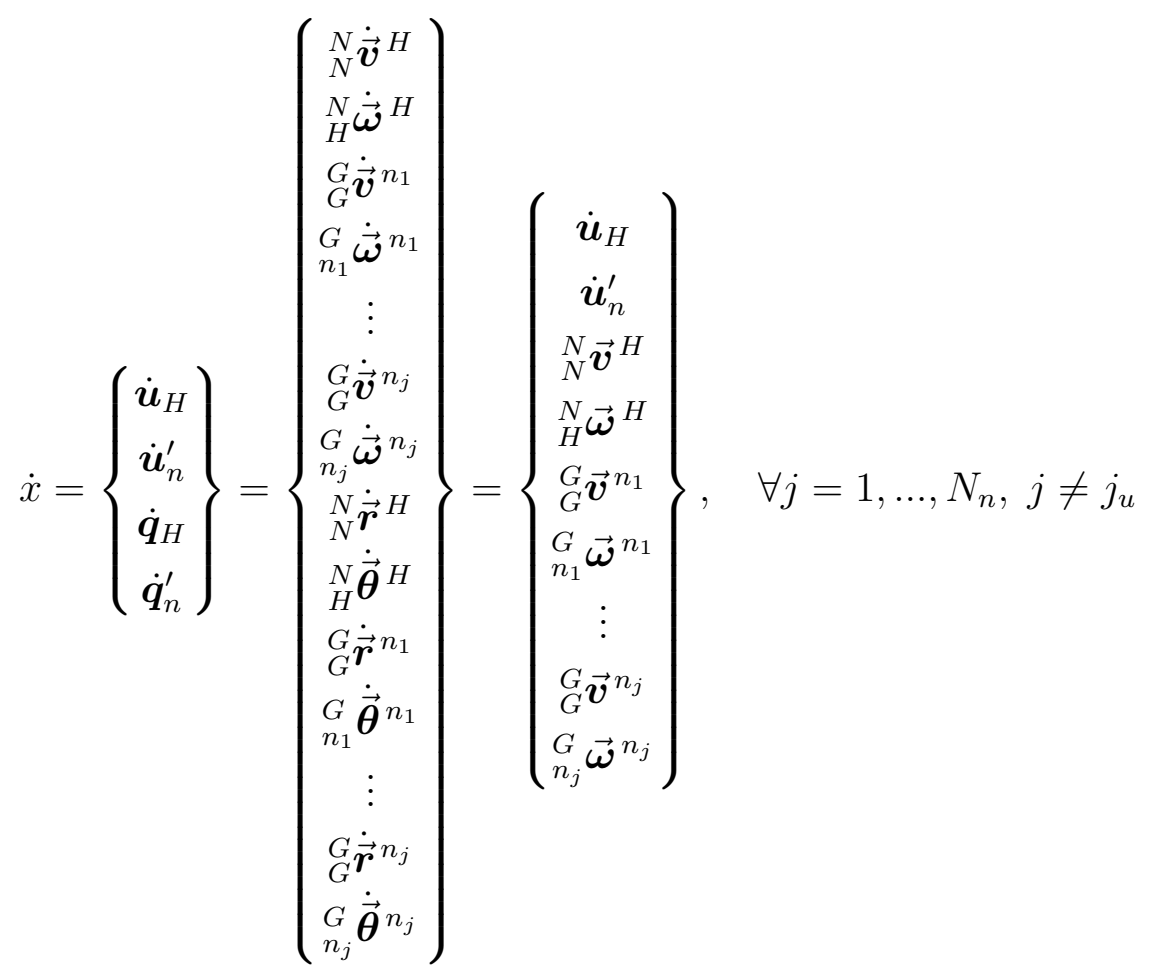

The solution vector in Equation 5.18 is propagated forward in time using numerical integration methods described in Section 5.2.1.

\subsection{Simulation Architecture}

SRAMSS was initially written in the interpreted programming language MATLAB ${ }^{\circledR}$ for its extensive mathematical libraries, plotting capabilities, and ease of code modification and verification. Once the architecture of SRAMSS was established and verified, the code was ported to the Intel ${ }^{\circledR}$ Fortran compiled language. Fortran is well suited to the large number of array and vector manipulations required by the SRAMSS code. The compiled Fortran code provides computational speeds orders of magnitude faster than MATLAB ${ }^{\circledR}$. Depending on the simulation scenario, these speed increases reduced simulation run-time to near-real-time values. In non-stiff state-propagation regimes, SRAMSS well exceeded the real-time threshold. Such performance was achieved on an Intel ${ }^{\circledR}$ i5-6600k CPU clocked at 4.10 Ghz. Extensive optimizations could potentially have SRAMSS cross the real-time threshold in all cases; a desirable characteristic for integration into real-time interactive simulation environments. 
While the dynamical system in SRAMSS can be reduced to a system of linear equations as presented in Section 5.1, the dynamics involved are highly non-linear. Intermittent contact requires the reformulation of the linear system at each internal time-step. In effect, SRAMSS is a 'switched' hybrid system that switches between in-contact, and no-contact dynamic system formulations [54]. while both regimes are linear and continuous, the switch between regimes is not. Further, in the case of the DFE model, they are also numerically stiff. Specific techniques are used to optimize performance and increase fidelity in modelling the included dynamic phenomena.

\subsubsection{State Propagation}

For state-propagation in SRAMSS, multiple considerations are made in the selection of the numerical integrator.

The first thing to be considered is the numerical-stiffness of the system developed in SRAMSS. The inclusion of a DFE model into the system causes numerical stiffness; the DFE model eigenvalues are significantly different in order of magnitude compared to those of the overall system. Large nodal forces and small nodal displacements would require extremely small time-steps for generic solvers. Secondary contributions to system stiffness arise from the unilateral contact forces applied to the rigid skidlanding gear which significantly change the system dynamics on contact. Third, the system is relatively non-stiff when out of contact with the environment. However, the selection of a stiff solver to address the stiffness considerations is inefficient in the 'no-contact' regime.

Based on these considerations, the 'DLSODAR.for' ordinary differential equation (ODE) solver was chosen for the Fortran version of SRAMSS [55, 56]. DLSODAR is a solver which automatically switches between a non-stiff explicit 'Adams' method and a stiff implicit BDF method, using an algorithm developed by Petzhold [57]. This addresses the third consideration by choosing the appropriate solver based on the current apparent stiffness of the system. In both cases, the implemented solvers are linear-multistep methods which increase efficiency by usage of previous state information. The BDF solver is particularly suited to the stiff contact regimes where the DFE model is undergoing transient deformation.

Additionally, DLSODAR provides 'root-finding' capabilities from Hiebert and Shampine [58], which are leveraged to address the second consideration. In the transitions between no-contact and in-contact states for the contacting nodes, the dynamics 
of the system explicitly change due to unilateral contact. Zero contact forces are generated in the 'no-contact' regime ${ }_{n}^{n} \boldsymbol{F}_{k}^{c}=0$, whereas the in-contact regime generates a non-zero contact force ${ }_{n}^{n} \boldsymbol{F}_{k}^{c} \neq 0$. The root function provided to DLSODAR by SRAMSS models the contact states of the contact nodes $n_{j_{c}}$. The contact states are formulated such that a root is found at contact or release there from. This root function allows the termination of the current solution time-step $\Delta t_{\text {sol }}$ at the time of contact event (or release) $t_{e}$ as shown in Figure 5.1. This termination, resulting in shorter solution time-step $\Delta t_{e}$, separates the stiff in-contact regime from the non-stiff no-contact regimes. DLSODAR is then able to switch between solvers which results in improved simulation performance around these transitions since increasingly small solver internal time-steps are avoided around these discontinuities.

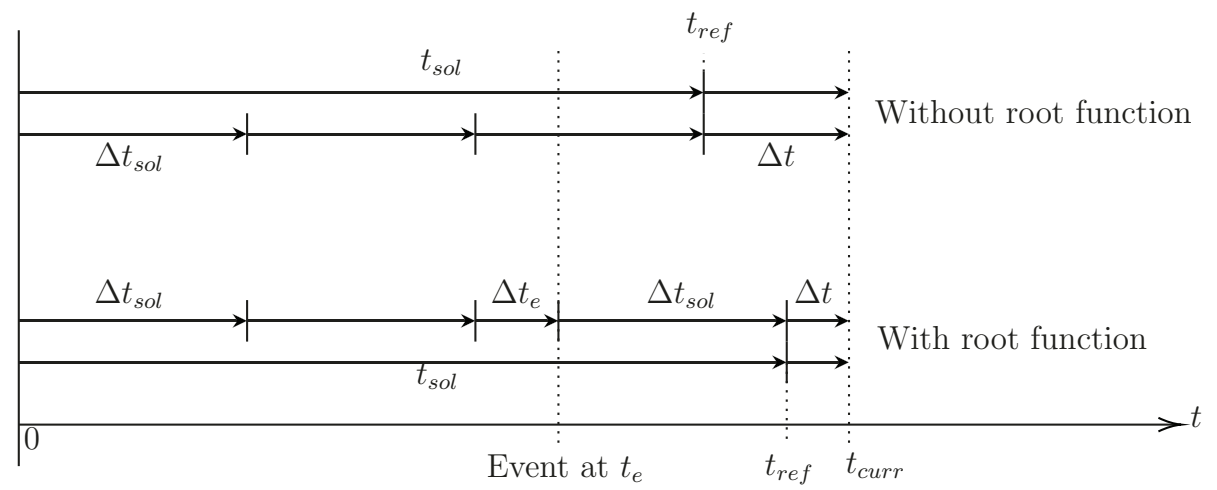

Figure 5.1: State propagation with root-finding step termination.

The root function algorithm employed in SRAMSS for contact event handling is developed in the following section.

\subsubsection{Contact Event Handling}

The instance where contact or release from contact occurs is called an 'event'. Timestep termination at these events makes SRAMSS' implementation of the DLSODAR solver partially event-driven. Detection of these events is handled by the contact detection algorithm implemented in SRAMSS. The algorithm is integrated into the SAT contact dynamics model to provide appropriate surface determination for dynamic response, and contact event detection. In each case, a scalar value is calculated. The index $m$ of polygon contact surface ${ }^{P} s_{m}$ is determined to apply contact forces in the correct direction. A scalar-valued root function ${ }^{P} g^{n_{j_{c}}}(t)$, representing the contactpair of node $n_{j_{c}}$ and polygon $P$, crosses zero at a contact event to enable solution 
time-step termination at $\Delta t_{e}$.

\section{Appropriate Surface Detection}

In Section 4.2 of Chapter 4, it was assumed that surface ${ }^{P} s_{m}$ was in contact with node $n_{j_{c}}$. The objective of the contact detection algorithm is to determine the value of index $m$ corresponding to the contact surface ${ }^{P} s_{m}$ of polygon $P$. The SAT algorithm inherently provides a straight-forward method for detecting surface collision by use of the surface normal axes.

Contact detection using the SAT first requires a binary penetration state matrix (PSM) $\left[{ }^{P} \boldsymbol{A}^{n_{j c}}(t)\right]$ which indicates penetration (no axis of separation) of contact node $n_{j_{c}}$ along surface ${ }^{P} s_{m}$ of polygon $P$ at time $t$ such that

$$
\begin{aligned}
& {\left[{ }^{P} \boldsymbol{A}^{n_{j_{c}}}(t)\right]=\left[\begin{array}{c}
\boldsymbol{A}_{s_{1}} \\
\vdots \\
\boldsymbol{A}_{s_{m}}
\end{array}\right]= \begin{cases}\boldsymbol{A}_{s_{m}}=0, & { }_{N}^{N} \overrightarrow{\boldsymbol{r}}^{n_{j_{c}}} \cdot{ }_{N}^{P} \hat{\boldsymbol{s}}_{m}>\left({ }_{N}^{N} \overrightarrow{\boldsymbol{r}}^{P_{m}} \cdot{ }_{N}^{P} \hat{\boldsymbol{s}}_{m}\right) \\
\boldsymbol{A}_{s_{m}}=1, & { }_{N}^{N} \overrightarrow{\boldsymbol{r}}^{n_{j_{c}}} \cdot{ }_{N}^{P} \hat{\boldsymbol{s}}_{m} \leq\left({ }_{N}^{N} \boldsymbol{r}^{A_{m}} \cdot{ }_{N} \hat{\boldsymbol{s}}_{m}\right)\end{cases} } \\
& m=1, \ldots N_{s, P}
\end{aligned}
$$

By Equation 4.37, penetration can be obtained by the projection of the contact node $n_{j_{c}}$ to the separation axis of surface ${ }^{P} s_{m}$. Since the surface normals ${ }_{N}^{P} \hat{\boldsymbol{s}}_{m}$ point outwards, there is no penetration along ${ }^{P} s_{m}\left(\boldsymbol{A}_{s_{m}}=0\right)$ when the projected node exceeds the projected polygon vertex, and penetration otherwise $\left(\boldsymbol{A}_{s_{m}}=1\right)$.

Next, by the existence of an arbitrary axis separating two objects as shown in Figure 4.3, contact only occurs when such an axis cannot be found. During contact, all surfaces are penetrated, $\left[{ }^{P} \boldsymbol{A}^{n_{j_{c}}}(t)\right]=1$. For the application of an appropriatelyoriented dynamic response, the surface involved in collision must be determined. The desired surface can be determined through monitoring of the transient penetration state changes in $\left[{ }^{P} \boldsymbol{A}^{n_{j_{c}}}(t)\right]$. Let the binary penetration state change matrix (PSCM) $\left[{ }^{P} \boldsymbol{B}^{n_{j_{c}}}(t)\right]$ indicate the change in penetration with surface ${ }^{P} s_{m}$ between reference time $t_{\text {ref }}$ and desired time $t$, such that

$$
\begin{aligned}
{\left[{ }^{P} \boldsymbol{B}^{n_{j_{c}}}(t)\right] } & =\left[{ }^{P} \boldsymbol{A}^{n_{j_{c}}}(t)\right]-\left[{ }^{P} \boldsymbol{A}^{n_{j_{c}}}\left(t_{r e f}\right)\right] \\
{\left[{ }^{P} \boldsymbol{B}^{n_{j_{c}}}\left(t_{c u r r}\right)\right] } & =\left[{ }^{P} \boldsymbol{A}^{n_{j_{c}}}\left(t_{c u r r}\right)\right]-\left[{ }^{P} \boldsymbol{A}^{n_{j_{c}}}\left(t_{\text {sol }}\right)\right]
\end{aligned}
$$


where

$$
\left[{ }^{P} \boldsymbol{B}^{n_{j_{c}}}(t)\right]=\left[\begin{array}{c}
\boldsymbol{B}_{s_{1}} \\
\vdots \\
\boldsymbol{B}_{s_{m}}
\end{array}\right], \quad \boldsymbol{B}_{s_{m}}=\left\{\begin{array}{cl}
1, & \text { into penetration } \\
0, & \text { no change } \\
-1, & \text { out of penetration }
\end{array}=1, \ldots, N_{s, P}\right.
$$

In the case of the linear multistep solver DLSODAR which aims to propagate the solution by a time-step increment of $\Delta t_{s o l}, t_{r e f}$ is chosen as the start time of the solver time-step $t_{\text {sol }}$. This reference time coincides with a solution propagated fully through $\Delta t_{\text {sol }}$ where the contact state has not changed, or the early termination at $\Delta t_{e}$, the time where an event was detected and the PSM takes on a new penetration state configuration.

The desired time is taken as $t_{c u r r}$ the time currently being evaluated by the solver. Usage of these definitions of times for the PSCM eliminates errors arising from failed internal time-steps, and aligns with the objective of using the root-finding function to start new solution time-steps at changes in collision states.

Along with the PSM and PSCM, a contact surface matrix (CSM) $\left[{ }^{P} \boldsymbol{D}^{n_{j_{c}}}(t)\right]$ is required. The CSM indicates which surface ${ }^{P} s_{m}$ is in contact at time $t$. The CSM determines if a change in penetration state indicated by the PSCM constitutes contact, or release from contact, with a polygon surface. The CSM at time $t$ is constructed

$$
\left[{ }^{P} \boldsymbol{D}^{n_{j_{c}}}(t)\right]=\left[\begin{array}{c}
\boldsymbol{D}_{s_{1}} \\
\vdots \\
\boldsymbol{D}_{s_{m}}
\end{array}\right], \quad \boldsymbol{D}_{s_{m}}=\left\{\begin{array}{ll}
0, & \text { not contact surface } \\
1, & \text { contact surface }
\end{array}=1, \ldots, N_{s, P}\right.
$$

For a given solver step $\Delta t_{s o l},\left[{ }^{P} \boldsymbol{D}^{n_{j_{c}}}\left(t_{s o l}\right)\right]$ is required to indicate the contact state at the beginning of the current solution time-step, and to determine if $\left[{ }^{P} \boldsymbol{B}^{n_{j_{c}}}\left(t_{c u r r}\right)\right]$ indicates a change with respect to the contact surface during the current solver step $\Delta t_{\text {sol }}$. The CSM at time $t_{\text {curr }}$ is then determined by the following algorithm:

$$
\begin{aligned}
\boldsymbol{D}_{s_{m}}\left(t_{c u r r}\right) & = \begin{cases}1, & \sum\left[{ }^{P} \boldsymbol{A}^{n_{j_{c}}}\left(t_{c u r r}\right)\right]=N_{s, P} \wedge \boldsymbol{B}_{s_{m}}\left(t_{c u r r}\right)=1 \\
\boldsymbol{D}_{s_{m}}\left(t_{\text {sol }}\right), & \sum\left[{ }^{P} \boldsymbol{A}^{n_{j_{c}}}\left(t_{c u r r}\right)\right]=N_{s, P} \wedge \boldsymbol{B}_{s_{m}}\left(t_{c u r r}\right)=0 \\
0, & \text { otherwise }\end{cases} \\
m & =1, \ldots, N_{s, P}
\end{aligned}
$$


where at the current time $t_{\text {curr }}$ if the summation of all elements of $\left[{ }^{P} \boldsymbol{A}^{n_{j_{c}}}\left(t_{c u r r}\right)\right] \neq$ $N_{s, P}$, then a separation axis exists since not all surfaces are penetrated, and the node is not in contact with any surface. As a result the CSM takes on the value $\left[{ }^{A} \boldsymbol{D}^{n_{j_{c}}}\left(t_{\text {curr }}\right)\right]=0$; no surface is in contact. If $\sum\left[{ }^{P} \boldsymbol{A}^{n_{j_{c}}}\left(t_{\text {curr }}\right)\right]=N_{s, P}$, then the PSCM element where $\boldsymbol{B}_{s_{m}}\left(t_{\text {curr }}\right)=1$ indicates a node coming into contact with surface ${ }^{P} s_{m}$ since the last penetration state $\boldsymbol{A}_{s_{m}}$ to change into penetration $\left(\boldsymbol{B}_{s_{m}}\left(t_{c u r r}\right)=1\right)$ is the contact surface. Otherwise the CSM maintains its $\Delta t_{\text {sol }}$ value since the PSM has not changed, i.e. the contact node remains in its current penetration state of ${ }^{P} s_{m}$. For $B_{m}\left(t_{c u r r}\right)=-1, \sum\left[{ }^{P} \boldsymbol{A}^{n_{j c}}\left(t_{c u r r}\right)\right]=N_{s, P}$ cannot be satisfied since -1 indicates coming out of penetration of surface ${ }^{P} s_{m}$ and the existence of a separation axis as before.

Then, for a CSM with $r$ elements, the index $m$ of the contact surface ${ }^{P} s_{m}$ of polygon $P$ is determined

$$
\left[{ }^{P} \boldsymbol{D}^{n_{j_{c}}}\left(t_{c u r r}\right)\right]=\left[\begin{array}{c}
\boldsymbol{D}_{s_{1}} \\
\vdots \\
\boldsymbol{D}_{s_{r}}
\end{array}\right], \text { where } \boldsymbol{D}_{s_{r}}\left(t_{c u r r}\right)=1: m=r
$$

If $\left[{ }^{P} \boldsymbol{D}^{n_{j_{c}}}\left(t_{c u r r}\right)\right]=0$ then $m$ cannot be found. Therefore node $n_{j_{c}}$ is not in contact with polygon $P$ and ${ }_{n_{j c}}^{n_{j c}} \overrightarrow{\boldsymbol{F}}^{P}=0$. Otherwise, where index $m$ of the contacted surface is found, application of the contact dynamics model along the appropriate surface for dynamic response is then possible.

This, the first objective of the contact detection algorithm provides the index $m$ of the contact surface ${ }^{P} s_{m}$ when in contact, which can then be fed to the contact dynamics model presented in Section 4.2 to apply the appropriately-oriented dynamic response. The precise time $t_{e}$ at which the contact state changes, and thusly the application of the contact forces, remains to be determined.

\section{Event root function}

As previously stated, the dynamics in SRAMSS change drastically when a change in contact state for node $n_{j_{c}}$ occurs. Providing a set of root functions ${ }^{P} g^{n_{j_{c}}}(t)$ for all contact-pairs to the DLSODAR solver allows for early termination of the solution time-step at $\Delta t_{e}$. The solution is propagated to $t_{e}=t_{\text {sol }}+\Delta t_{e}$, where $t_{e}$ is the contact event time where one of the provided root functions crosses zero.

Each root function in the set provided to DLSODAR crosses zero at the eventtime where a contact-pair changes contact state. Definition of the root functions in 
this manner splits the unilateral dynamics into two separate and smooth regimes. On one side, in the 'no-contact' regime, the contact forces for the $j_{c}$ th contact node

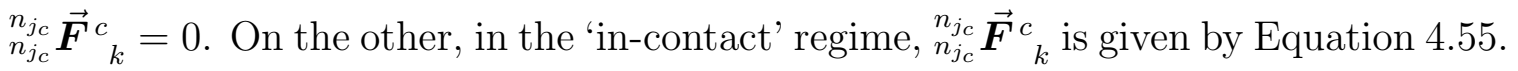
At initial contact of surface ${ }^{A} S_{m}, \Delta_{p}$ from Equation 4.39 equals zero. Therefore the root function can be defined using distances to the contact surface ${ }^{P} s_{m}$.

For the in-contact regime, the indice $m$ is known, thus the root function can be expressed using the penetration depth ${ }^{s_{m}} \Delta_{p}$ along surface normal ${ }_{N}^{P} \hat{\boldsymbol{s}}_{m}$. For the nocontact regime, the distance must be assumed. Initial contact is assumed to occur at the closest surface to node $n_{j_{c}}$. The closest surface is determined by the minimum positive value of ${ }^{s_{m}} \Delta_{p}$ for all surfaces $m=1, \ldots, N_{s, P}$ belonging to polygon $P$, given by Equation 4.39. The root function for a contact pair is then expressed as

$$
{ }^{P} g^{n_{j_{c}}}\left(t_{\text {curr }}\right)= \begin{cases}{ }^{s_{m}} \Delta_{p}, & \boldsymbol{D}_{S_{m}}\left(t_{\text {curr }}\right)=1 \\ \min \left({ }^{s_{m}} \Delta_{p}>0\right), & {\left[{ }^{P} \boldsymbol{D}^{\left.n_{j_{c}}\left(t_{\text {curr }}\right)\right]=0}\right.}\end{cases}
$$

This Section completes the definitions of the contact dynamics, and numerical integration method used in SRAMSS. The next section presents an overview of the SRAMSS architecture as a whole.

\subsubsection{Subroutine Architecture}

SRAMSS can be described as a variable-output-time-step simulation which propagates the solution vector forwards in time by use of an event-driven linear multistep ODE solver. Figure 5.2 shows a high-level representation of the SRAMSS software architecture.

Per note 1 in Figure 5.2, SRAMSS requires the following input files, the contents of which are described in Appendix A.1:

- Simulation.inp: Simulation parameters;

- Output_options.inp: flag file for output files desired;

- ICs.inp: generalized coordinate and speed initial conditions;

- Properties_helo.inp: Helicopter properties;

- Properties_contact_mech.inp: contact mechanics properties;

- Properties_FE_map.inp: DFE node-element mapping, and elemental properties;

- Properties_FE_IC.inp: Undeformed DFE node mesh ${ }_{G}^{G} \overrightarrow{\boldsymbol{r}}^{n_{j}}{ }_{0}$ and ${ }_{G}^{G} \overrightarrow{\boldsymbol{\theta}}^{n_{j}}$;

- Properties_FE_Mmat.inp: externally-generated DFE mass matrix (optional); 


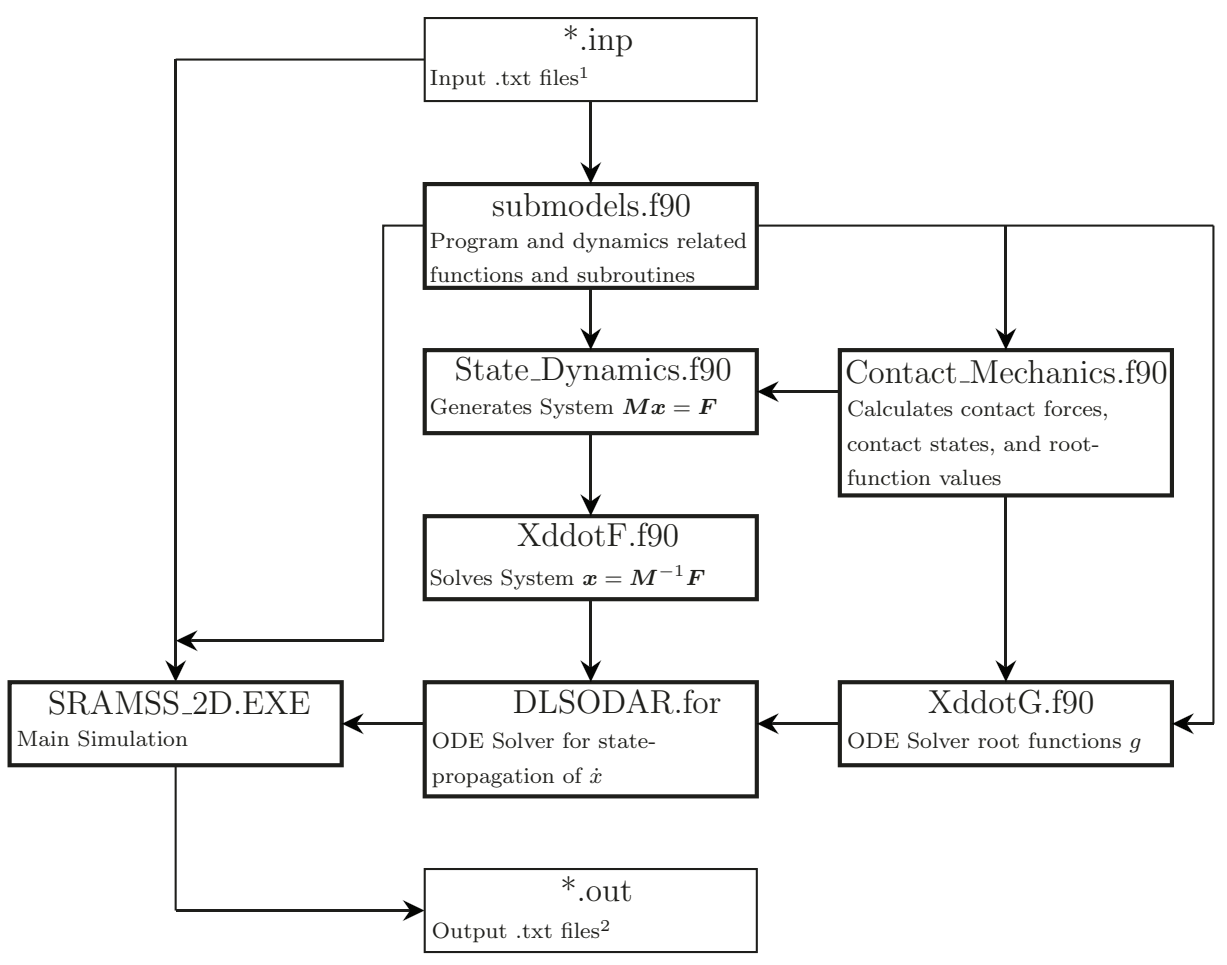

Figure 5.2: SRAMSS program architecture.

- Properties_FE_Kmat.inp: externally-generated total structure stiffness matrix (optional);

- Properties_FE_Cmat.inp: externally-generated total structure proportional damping matrix (optional);

- Polygon_ship.inp: Ship polygon vertex positions ${ }_{S}^{S} \overrightarrow{\boldsymbol{r}} S_{m}$;

- Polygon_rsd.inp: RSD deck polygon vertex positions ${ }_{D}^{D} \overrightarrow{\boldsymbol{r}} D_{m}$;

- Polygon_clampleft.inp: Left wedge clamp polygon vertex positions ${ }_{C L}^{C L} \overrightarrow{\boldsymbol{r}} C L_{m}$;

- Polygon_clampright.inp: Right wedge clamp polygon vertex positions ${ }_{C R}^{C R} \overrightarrow{\boldsymbol{r}}^{C R_{m}}$;

- Prescribed_ship.inp: prescribed motion for S-frame;

- Prescribed_rsd.inp: prescribed motion for D-frame;

- Prescribed_clampleft.inp: prescribed motion for $C L$-frame;

- Prescribed_clampright.inp: prescribed motion for CR-frame; and

- Prescribed_aero.inp: prescribed aerodynamics including wind velocity ${ }_{N}^{N} \overrightarrow{\boldsymbol{v}}^{W}$, rotor RPM ${ }_{R}^{H} \overrightarrow{\boldsymbol{\omega}}^{R}$, collective pitch setting $\theta_{\text {coll }}$, and cyclic pitch settings $\theta_{c y c, \text { max }}$ and $\psi_{\max }$.

Per note 2 in Figure 5.2, SRAMSS can currently generate the following output files where appropriately flagged in Output_options.inp, the contents of which are described 
in Appendix A.2:

- GEN_SOLUTION.out: ouput time-stamped solution vectors of generalized speeds and coordinates;

- POLY_SHIP.out: output time-stamped ship polygon vertices ${ }_{N}^{N} \overrightarrow{\boldsymbol{r}}^{S_{m}}$;

- POLY_RSD.out: output time-stamped RSD deck polygon vertices ${ }_{N}^{N} \overrightarrow{\boldsymbol{r}}^{D_{m}}$;

- $P O L Y_{-} L_{-} C L A M P$.out: output time-stamped left wedge clamp polygon vertices ${ }_{N}^{N} \overrightarrow{\boldsymbol{r}}^{C L_{m}}$;

- $P O L Y_{-} R_{-} C L A M P$.out: output time-stamped right wedge clamp polygon vertices ${ }_{N}^{N} \overrightarrow{\boldsymbol{r}}^{C R_{m}}$;

- OCP_SHIP.out: output time-stamped ship polygon OCP for all contact nodes ${ }_{N}^{S} \overrightarrow{\boldsymbol{o}}^{n_{j_{c}}}$

- OCP_RSD.out: output time-stamped RSD deck OCP for all contact nodes ${ }_{N}^{D} \overrightarrow{\boldsymbol{o}}^{n_{j_{c}}}$;

- OCP_L_CLAMP.out: output time-stamped left wedge clamp OCP for all contact nodes ${ }_{N}^{C L} \overrightarrow{\boldsymbol{o}}^{n_{j c}}$;

- $O C P_{-} R_{-} C L A M P$.out: output time-stamped right wedge clamp OCP for all contact nodes ${ }_{N}^{C R} \overrightarrow{\boldsymbol{o}}^{n_{j_{c}}}$

- FORCE_CONTACT.out: output time-stamped contact forces for all contact $\operatorname{nodes}{ }_{N}^{n_{j_{c}}} \boldsymbol{F}_{k}^{c}$;

- FORCE_INDUCED.out: output time-stamped rotor disc induced forces ${ }_{H}^{H} \overrightarrow{\boldsymbol{F}}^{B E M}$ and ${ }_{H}^{H} \overrightarrow{\boldsymbol{M}}^{B E M}$; and

- SIM_STATS.out: output time-stamped simulation and 'DLSODAR.for' statistics. 


\section{Chapter 6}

\section{SRAMSS Verification and Validation}

Dynamic simulation packages such as Dynaface ${ }^{\circledR}$, SSMASH, and SRAMSS, serve a unique purpose in cost-effectively performing faithful dynamic interface analyses without the need for full-scale experiments. Yet the accuracy of these packages is bounded by the fidelity of implemented virtual models. Accuracy must then be measured. For SRAMSS, this involves the verification of proper implementation of model formulas and algorithms, and the validation of simulation results against experimental data and known physical theories and laws. For the planar case of SRAMSS developed in this work, validation is made mostly by comparison with theories and laws since the planar simulation environment cannot capture the out-of-plane effect present in fully-spatial commercially available dynamic interface packages.

This chapter discusses the verification and validation of SRAMSS. The generic aircraft used for simulation test cases, and the description of these test cases follow in Sections 6.1 and 6.2 respectively. Results from the test cases are discussed in Section 6.3. Finally, Section 6.4 provides other general remarks and observations made for SRAMSS.

\subsection{Generic Aircraft Description}

To ensure consistency between the test cases presented in the following section, a sin-

gle generic aircraft is defined. The generic aircraft definition and parameters remain constant for all test cases, unless a given test case is specifically evaluating the effect 
of a parameter variation. For example, the DFE model mesh may vary for a test case to observe convergence as the DFE model mesh is refined.

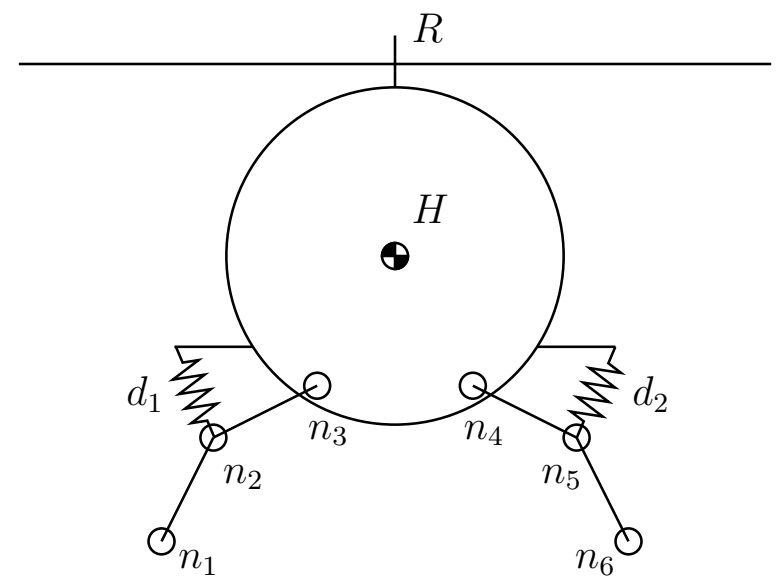

Figure 6.1: Generic aircraft used in verification an validation test cases.

The generic aircraft used for verification purposes is as shown in Figure 6.1. The aircraft body is composed of a single rigid-body $H$ representing the airframe, and a 6-node DFE model representing the left and right legs of a skid-type landing gear. In addition, the generic aircraft has two discrete external dampers attached between the middle nodes of the DFE landing gear legs and the airframe's winglets. There is also a rotor disc $R$ with rectangular blades located on the top-side of the airframe.

For the complete description of the aircraft,

- Table 6.1 presents the physical parameters of the aircraft;

- Table 6.2 describes the functionality of each DFE node by node index $j$;

- Table 6.3 and Figure 6.2 present the rotor disc BEM model parameters; and

- Table 6.4 presents the contact dynamics properties.

From Figure 6.2, the Mach number effects from Equation 4.72 are ignored by setting the thickness ratio to zero. Since the drag coefficient is set to zero, rotor blade drag is also effectively not considered. 
Table 6.1: Generic aircraft parameters

\begin{tabular}{lcc}
\hline Parameter & Variable & Value \\
\hline Airframe Parameters & & \\
Airframe mass & $m_{H}$ & $50 \mathrm{~kg}$ \\
Airframe mass moment of inertia & $I_{H}$ & $6.25 \mathrm{~kg} \cdot \mathrm{m}^{2}$ \\
Discrete damper spring coefficient & $K_{d}$ & $2.5 \mathrm{X} 10^{4} \mathrm{~N} / \mathrm{m}$ \\
Discrete damper damping coefficient & $C_{d}$ & $500 \mathrm{Ns} / \mathrm{m}$ \\
Equivalent flat plate area & $\boldsymbol{A}_{e q}$ & $\{222\}^{\mathrm{T}} \mathrm{m}^{2}$ \\
Centre of pressure & ${ }_{H} \overrightarrow{\boldsymbol{r}}^{C P}$ & $\{0-0.50\}^{\mathrm{T}} \mathrm{m}$ \\
\hline DFE Model Landing Gear Parameters & & \\
Number of nodes & $N_{n}$ & 6 \\
Number of elements & & 4 \\
Element Young's modulus & $E$ & $6.9 \mathrm{X} 10^{10} \mathrm{~Pa}$ \\
Element second moment of area & $I$ & $5.00 \mathrm{X} 10^{-7} \mathrm{~m}^{4}$ \\
Element density & $\rho$ & $2710 \mathrm{~kg} / \mathrm{m}^{3}$ \\
Element cross-section & $A$ & $1.2 \times 10^{-4} \mathrm{~m}^{2}$ \\
Rayleigh damping lower frequency & $\omega_{1}$ & $0 \mathrm{rad} / \mathrm{s}$ \\
Rayleigh damping lower damping ratio & $\xi_{1}$ & 0.15 \\
Rayleigh damping upper frequency & $\omega_{2}$ & $6.5 \mathrm{X} 10^{5} \mathrm{rad} / \mathrm{s}$ \\
Rayleigh damping upper damping ratio & $\xi_{2}$ & 0.15 \\
Skid-tube cross-section radius & & $0.01 \mathrm{~m}$ \\
\hline
\end{tabular}

Table 6.2: DFE node functionality for generic aircraft

\begin{tabular}{ccc}
\hline Node Type & Node Variable & Set of Indices \\
\hline All & $n j$ & $j=\{1,2,3,4,5,6\}$ \\
Contact & $n_{j_{c}}$ & $j_{c}=\{1,6\}$ \\
Interface & $n_{j_{u}}$ & $j_{u}=\{3,4\}$ \\
Damper & $n_{j_{d} n}$ & $j_{n}=\{2,5\}$ \\
\hline
\end{tabular}


Table 6.3: Rotor disc blade element model parameters

\begin{tabular}{lcc}
\hline Parameter & Variable & Value \\
\hline Number of rotor blades & $N_{b}$ & 4 \\
Number of blade elements & $N_{e}$ & 80 \\
Number of rotor disc sectors & $N_{\psi}$ & 20 \\
Rotor blade radius & $R_{b}$ & $3 \mathrm{~m}$ \\
Inner effective rotor disc radius & $R_{i}$ & 0.2 \\
Outer effective rotor disc radius & $R_{o}$ & 0.9 \\
Blade chord length & $c$ & Fig.6.2 \\
Blade thickness ratio & $t / c$ & \\
Blade twist & $\theta_{t}$ & \\
Uncorrected lift coefficient gradient & $\frac{\mathrm{d} C_{L}}{\mathrm{~d} \alpha}$ & \\
Uncorrected lift coefficient at $\alpha=0$ & $C_{L, 0}$ & \\
Uncorrected drag coefficient gradient & $\frac{\mathrm{d} C_{D}}{\mathrm{~d} \alpha}$ & \\
Uncorrected drag coefficient $\alpha=0$ & $C_{D, 0}$ & $\downarrow$ \\
\hline
\end{tabular}

Table 6.4: Contact dynamics parameters

\begin{tabular}{lcc}
\hline Parameter & Variable & Value \\
\hline Penetration stiffness & $K_{p}$ & $1 \times 10^{6} \mathrm{~N} / \mathrm{m}$ \\
Penetration damping & $C_{p}$ & $3130 \mathrm{Ns} / \mathrm{m}$ \\
Friction micro-displacement stiffness & $K_{f}$ & $3 \times 10^{3} \mathrm{~N} / \mathrm{m}$ \\
Friction micro-displacement damping & $C_{f}$ & $600 \mathrm{Ns} / \mathrm{m}$ \\
Stribeck velocity threshold & $v_{s}$ & $0.001 \mathrm{~m} / \mathrm{s}$ \\
Dynamic friction coefficient & $\mu_{d}$ & 0.4 \\
Static friction coefficient & $\mu_{s}$ & 0.9 \\
\hline
\end{tabular}



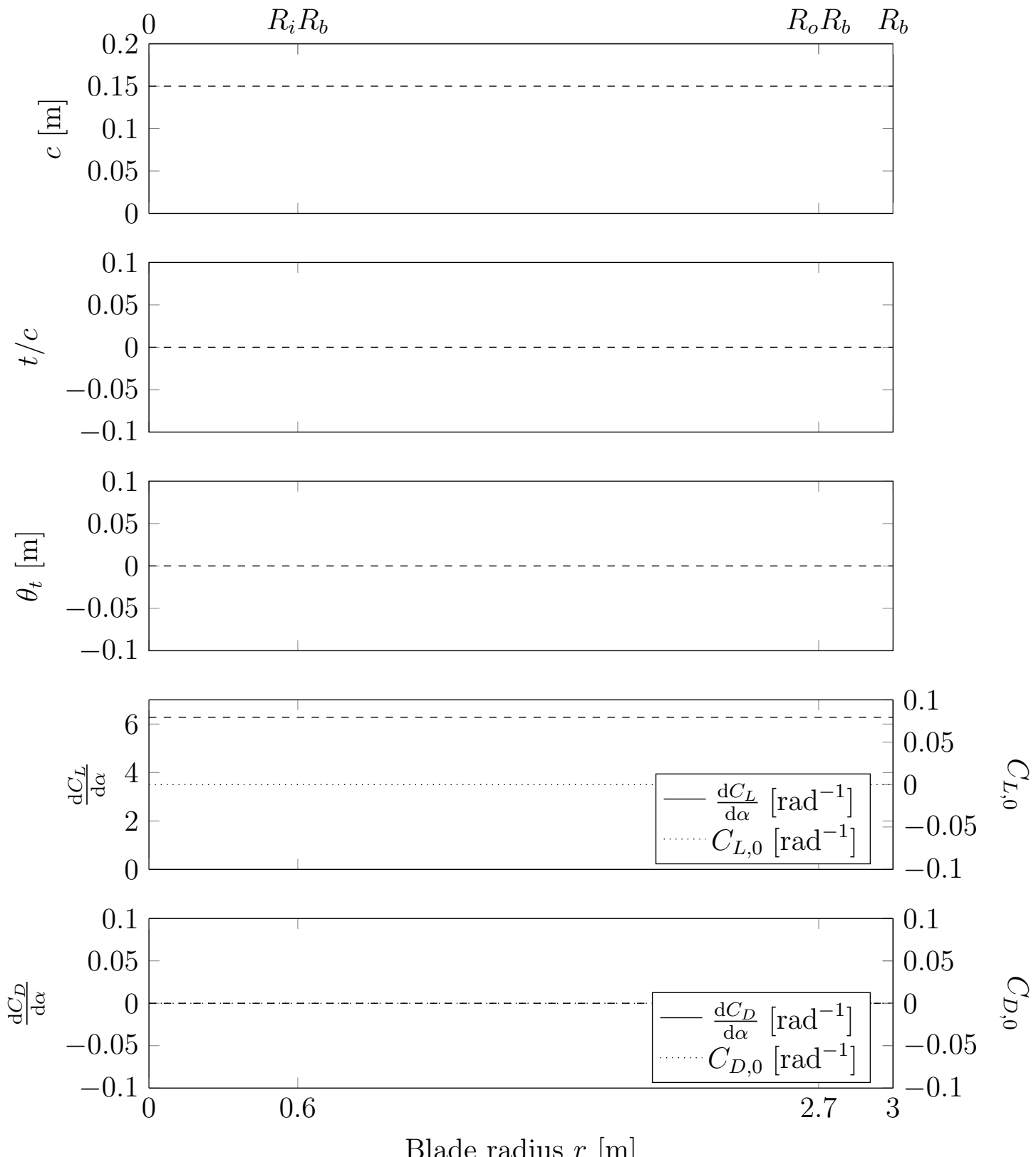

Figure 6.2: Generic aircraft blade profile. 


\subsection{Test Cases Description}

While the 2D version of SRAMSS discussed in this work cannot capture the out-ofplane effects present in a complete 3D simulation, it can be used to verify planar test cases to verify the equations of motion and models included in SRAMSS.

SRAMSS is composed of many complex models which generate a wide variety of dynamic phenomena. The test cases are designed to isolate and address implemented models individually such that the implementation of each model can be independently verified. Additionally, the isolation of the models allows validation against known theories and experimental data.

For this work, there are many models which must be verified:

- the correct implementation of gravity in the gravitational model;

- the forces generated in the external damper model;

- interfacing the rigid airframe to the flexible landing gear, and the dynamic response of the DFE model;

- the appropriately-oriented dynamic response of the contact dynamics model;

- the response of the aircraft in cross-winds and the induced thrust of the rotor disc in the aerodynamic models; and

- and the preliminary design of the skid securing system.

Beyond verification, some of these models can also be validated against known physical theories and experimental data. Static equilibrium can be validated when the aircraft comes to rest, gravitational acceleration can be validated in free fall, and the Rotor BEM can be validated against experimental data where blade geometry is provided since the model exists in 3D.

The test cases developed to evaluate the planar case of SRAMSS are presented in Table 6.5. 
Table 6.5: SRAMSS verification and validation test cases

\begin{tabular}{|c|c|c|c|}
\hline Test Case & Configuration & Objectives & Results \\
\hline $\begin{array}{l}\text { 1. Gravitational } \\
\text { model }\end{array}$ & & $\begin{array}{l}\text { Verify gravitational } \\
\text { acceleration and } \\
\text { numerical integration } \\
\text { implemented correctly. } \\
\text { - Verify on-deck deformed } \\
\text { steady-state posture. }\end{array}$ & $\begin{array}{l}\text { - Refer to Figure } 6.3 \text { in } \\
\text { Section } 6.3 .1 \text { for } \\
\text { acceleration plot. } \\
\text { - Refer to Table } 6.6 \text { in } \\
\text { Section } 6.3 .1 \text { for } \\
\text { deformed aircraft values. }\end{array}$ \\
\hline $\begin{array}{l}\text { 2. External damper } \\
\text { model }\end{array}$ & $\begin{array}{l}\mathrm{K} \uparrow \mathrm{C} \downarrow \\
\mathrm{K} \uparrow \mathrm{C} \uparrow \\
\mathrm{K} \downarrow \mathrm{C} \downarrow \\
\mathrm{K} \downarrow \mathrm{C} \uparrow \\
\end{array}$ & $\begin{array}{l}\text { - Verify behaviour of } \\
\text { aircraft landing with } \\
\text { varied damper stiffness } \\
\text { and damping } \\
\text { parameters. }\end{array}$ & $\begin{array}{l}\text { - Refer to Figures } 6.4 \\
\text { through } 6.6 \text { in } \\
\text { Section } 6.3 .2 \text { for } \\
\text { transient aircraft } \\
\text { behaviour. }\end{array}$ \\
\hline 3. Aircraft CoG & & $\begin{array}{l}\text { - Verify aircraft posture as } \\
\text { CoG is shifted } \\
\text { horizontally. } \\
\text { - Validate load } \\
\text { distribution on landing } \\
\text { gear and vertical static } \\
\text { equilibrium. }\end{array}$ & $\begin{array}{l}\text { - Refer to Figures } 6.7 \\
\text { and } 6.8 \text { in Section } 6.3 .3 \\
\text { for airframe roll angles. } \\
\text { - Refer to Figure } 6.9 \text { in } \\
\text { Section } 6.3 .3 \text { for landing } \\
\text { gear load distribution } \\
\text { and static equilibrium } \\
\text { validation. }\end{array}$ \\
\hline $\begin{array}{l}4.1 \text { Fixed DFE } \\
\text { landing gear }\end{array}$ & & $\begin{array}{l}\text { - Verify fixed interface } \\
\text { node behavior. } \\
\text { - Verify implementation of } \\
\text { DFE model and } \\
\text { damping matrix. } \\
\text { - Verify convergence of } \\
\text { DFE model with fixed } \\
\text { skid landing gear. }\end{array}$ & $\begin{array}{l}\text { - Refer to Figure } 6.11 \text { in } \\
\text { Section } 6.3 .4 \text { for aircraft } \\
\text { transient behaviour. }\end{array}$ \\
\hline
\end{tabular}


Table 6.5: (continued)

\begin{tabular}{|c|c|c|c|}
\hline Test Case & Configuration & Objectives & Results \\
\hline $\begin{array}{l}4.2 \text { Hinged DFE } \\
\text { landing gear }\end{array}$ & & $\begin{array}{l}\text { - Verify hinged interface } \\
\text { node behavior. } \\
\text { - Verify convergence of } \\
\text { DFE model with hinged, } \\
\text { externally-dampened } \\
\text { landing gear. } \\
\text { - Compare dominance of } \\
\text { external damping of } \\
\text { hinged landing gear } \\
\text { against fixed landing } \\
\text { gear. }\end{array}$ & $\begin{array}{l}\text { - Refer to Figure } 6.13 \text { in } \\
\text { Section } 6.3 .4 \text { for aircraft } \\
\text { transient behaviour. }\end{array}$ \\
\hline $\begin{array}{l}5.1 \text { Contact normal } \\
\text { forces }\end{array}$ & $\mathrm{F}_{\mathrm{N}}$ & $\begin{array}{l}\text { - Verify normal force } \\
\text { generation while at rest } \\
\text { on polygons. } \\
\text { - Validate normal force } \\
\text { generation in static } \\
\text { conditions. }\end{array}$ & $\begin{array}{l}\text { - Refer to Table } 6.9 \text { in } \\
\text { Section } 6.3 .5 \text { for } \\
\text { equilibrium force } \\
\text { analysis. }\end{array}$ \\
\hline $\begin{array}{l}5.2 \text { Contact friction } \\
\text { forces }\end{array}$ & & $\begin{array}{l}\text { Verify the behaviour of } \\
\text { LuGre model. } \\
\text { - Compare LuGre friction } \\
\text { model to simple } \\
\text { Coulomb model for } \\
\text { progressively-increasing } \\
\text { ship roll angle. }\end{array}$ & $\begin{array}{l}\text { - Refer to Figure } 6.15 \text { in } \\
\text { Section } 6.3 .5 \text { for sliding } \\
\text { velocity plot. } \\
\text { - Refer to Figure } 6.16 \text { in } \\
\text { Section } 6.3 .5 \text { for } \\
\text { transient friction forces } \\
\text { plot. }\end{array}$ \\
\hline $\begin{array}{l}\text { 5.3 Oriented } \\
\text { dynamic response }\end{array}$ & & $\begin{array}{l}\text { Verify appropriately } \\
\text { oriented dynamic } \\
\text { response of the contact } \\
\text { dynamics model. } \\
\text { - Verify aircraft-polygon } \\
\text { interaction behaves as } \\
\text { predicted. }\end{array}$ & $\begin{array}{l}\text { - Refer to Figure } 6.18 \\
\text { through } 6.20 \text { in } \\
\text { Section } 6.3 .5 \text { for aircraft } \\
\text { behaviour plot in } \\
\text { different simulation } \\
\text { scenarios. }\end{array}$ \\
\hline
\end{tabular}


Table 6.5: (continued)

\begin{tabular}{|c|c|c|c|}
\hline Test Case & Configuration & Objectives & Results \\
\hline $\begin{array}{l}6.1 \text { Aerodynamic } \\
\text { body drag forces }\end{array}$ & $\vec{\overrightarrow{ }}$ & $\begin{array}{l}\text { - Verify aerodynamic drag } \\
\text { forces in horizontal } \\
\text { direction due to relative } \\
\text { wind. } \\
\text { - Verify aerodynamic drag } \\
\text { forces in vertical } \\
\text { direction due to air } \\
\text { resistance. }\end{array}$ & $\begin{array}{l}\text { - Refer to Figure } 6.21 \text { in } \\
\text { Section } 6.3 .6 \text { for } \\
\text { translational velocities } \\
\text { plot. } \\
\text { - } \text { Refer to Figure } 6.22 \text { in } \\
\text { Section } 6.3 .6 \text { for vertical } \\
\text { acceleration plot. }\end{array}$ \\
\hline $\begin{array}{l}6.2 \text { Rotor thrust } \\
\text { without wind }\end{array}$ & $\mathrm{T} \uparrow$ & $\begin{array}{l}\text { - Verify that the BEM is } \\
\text { implemented correctly. } \\
\text { - Verify aircraft will } \\
\text { generate uniform thrust } \\
\text { in zero wind conditions. }\end{array}$ & $\begin{array}{l}\text { - Refer to Figure } 6.23 \text { in } \\
\text { Section } 6.3 .6 \text { for thrust } \\
\text { and vertical } \\
\text { displacement plots. } \\
\text { - Refer to Figure B.1 in } \\
\text { Section B.1 for the } \\
\text { time-varying BEM } \\
\text { parameters. }\end{array}$ \\
\hline $\begin{array}{l}\text { 6.3 Rotor thrust } \\
\text { with head-wind }\end{array}$ & $\frac{\overbrace{0}^{\mathrm{T}})^{\mathrm{M}}}{\text { Headwind }}$ & $\begin{array}{l}\text { - Continuation of BEM } \\
\text { verification. } \\
\text { - Verify non-uniform } \\
\text { thrust and moment } \\
\text { generation in headwinds. }\end{array}$ & $\begin{array}{l}\text { - Refer to Figure } 6.24 \text { in } \\
\text { Section } 6.3 .6 \text { for thrust, } \\
\text { rolling moment, and } \\
\text { airframe displacement } \\
\text { plots. } \\
\text { - Refer to Figure B.2 in } \\
\text { Section B.2 for the } \\
\text { time-varying BEM } \\
\text { parameters. }\end{array}$ \\
\hline $\begin{array}{l}6.4 \text { Rotor thrust } \\
\text { with head-wind } \\
\text { and cyclic } \\
\text { compensation }\end{array}$ & $\frac{\theta_{\text {cyc }}(\psi) \neq 0}{\text { Headwind }}$ & $\begin{array}{l}\text { - Continuation of BEM } \\
\text { verification. } \\
\text { - Verify cyclic pitch } \\
\text { setting compensation on } \\
\text { retreating blades side } \\
\text { generates enough thrust } \\
\text { to counter rolling } \\
\text { moment on aircraft in } \\
\text { the same test } \\
\text { environment as } \\
\text { Sub-case } 6.3 \text {. } \\
\text { - Refer to Figure B.3 in } \\
\text { Section B.3 for the } \\
\text { time-varying BEM } \\
\text { parameters. }\end{array}$ & $\begin{array}{l}\text { - Refer to Figure } 6.25 \text { in } \\
\text { Section } 6.3 .6 \text { for thrust, } \\
\text { rolling moment, and } \\
\text { airframe displacement } \\
\text { plots. }\end{array}$ \\
\hline
\end{tabular}


Table 6.5: (continued)

\begin{tabular}{|c|c|c|c|c|}
\hline & Test Case & Configuration & Objectives & Results \\
\hline & BEM validation & & $\begin{array}{l}\text { Validate BEM model } \\
\text { against CFD simulations } \\
\text { and experimentally } \\
\text { obtained data } \\
\text { - Refer to Figure C. } 3 \text { and } \\
\text { Table C. } 1 \text { in Section C.2 } \\
\text { for the BEM parameters. }\end{array}$ & $\begin{array}{l}\text { - Refer to Figure } 6.26 \text { in } \\
\text { Section } 6.3 .6 \text { for blade } \\
\text { sectional thrust } \\
\text { coefficients comparison. } \\
\text { - Refer to } \\
\text { Figures } 6.286 .27 \text { and in } \\
\text { Section } 6.3 .6 \text { aircraft } \\
\text { figure of merit } \\
\text { comparison. }\end{array}$ \\
\hline 7.1 & $\begin{array}{l}\text { Static aircraft } \\
\text { securing }\end{array}$ & & $\begin{array}{l}\text { - Verify implementation of } \\
\text { RSD deck securing } \\
\text { device. } \\
\text { - Verify aircraft's response } \\
\text { to securing mechanism } \\
\text { under static conditions. }\end{array}$ & $\begin{array}{l}\text { - Refer to Figures } 6.30 \\
\text { through } 6.32 \text { in } \\
\text { Section } 6.3 .7 \text { for securing } \\
\text { performance in static } \\
\text { scenarios. }\end{array}$ \\
\hline 7.2 & $\begin{array}{l}\text { Dynamic aircraft } \\
\text { securing }\end{array}$ & & $\begin{array}{l}\text { - Verify aircraft's response } \\
\text { to securing mechanism } \\
\text { under dynamic } \\
\text { conditions. }\end{array}$ & $\begin{array}{l}\text { - Refer to Figures } 6.34 \\
\text { and } 6.35 \text { in Section } 6.3 .7 \\
\text { for securing performance } \\
\text { in dynamic scenarios. } \\
\text { - Refer to Figures } 6.36 \\
\text { and } 6.37 \text { in Section } 6.3 .7 \\
\text { for securing device } \\
\text { contact node loads. }\end{array}$ \\
\hline 8. & $\begin{array}{l}\text { Comprehensive } \\
\text { Dynamic } \\
\text { Phenomena }\end{array}$ & $\leftarrow$ & $\begin{array}{l}\text { - Verify aircraft behavior } \\
\text { under fully-dynamic } \\
\text { conditions. } \\
\text { - Verify collision behavior } \\
\text { with multiple objects } \\
\text { (RSD and ship deck). } \\
\text { - Compare impact of DFE } \\
\text { and BEM mesh } \\
\text { refinements. } \\
\text { - RSD deck and aircraft } \\
\text { have non-zero initial } \\
\text { velocity conditions. } \\
\text { - Refer to Figure D.1 } \\
\text { and D.2 in Section D for } \\
\text { the time-varying BEM } \\
\text { parameters, and polygon } \\
\text { prescribed motion. }\end{array}$ & $\begin{array}{l}\text { - Refer to Figure } 6.38 \text { in } \\
\text { Section } 6.3 .8 \text { for } \\
\text { significant simulation } \\
\text { event animations. } \\
\text { - Refer to Figure } 6.39 \text { in } \\
\text { Section } 6.3 .8 \text { for airframe } \\
\text { response. } \\
\text { - Refer to Figures } 6.40 \\
\text { through } 6.41 \text { in } \\
\text { Section } 6.3 .8 \text { for securing } \\
\text { performance and contact } \\
\text { node loads in dynamic } \\
\text { scenarios. } \\
\text { - Refer to Figure } 6.39 \text { in } \\
\text { Section } 6.3 .8 \text { for rotor } \\
\text { disc forces. }\end{array}$ \\
\hline
\end{tabular}




\subsection{Test Cases Verification and Validation}

The following sections present the observations and results for each set of test cases, and discuss their relevance to verification and validation of the planar case of SRAMSS.

\subsubsection{Case 1: Gravitational Model}

The first test case is a level drop from $0.8 \mathrm{~m}$ of the generic aircraft towards the RSD deck with zero-velocity initial conditions. Aerodynamic models and contact friction models are turned off for this test case. Figure 6.3 shows the airframe's CoG position as the solid line, acceleration as the dot-dashed line, and gravitational acceleration as the dotted line. While in the air, the aircraft maintains a downwards acceleration of $9.81 \mathrm{~m} / \mathrm{s}^{2}$. When the aircraft's CoG falls below 0.5500 metres (in contact with RSD deck) the acceleration value deviates from $9.81 \mathrm{~m} / \mathrm{s}^{2}$. At steady-state, the acceleration settles to $0 \mathrm{~m} / \mathrm{s}^{2}$. This test verifies the correct implementation of gravity.

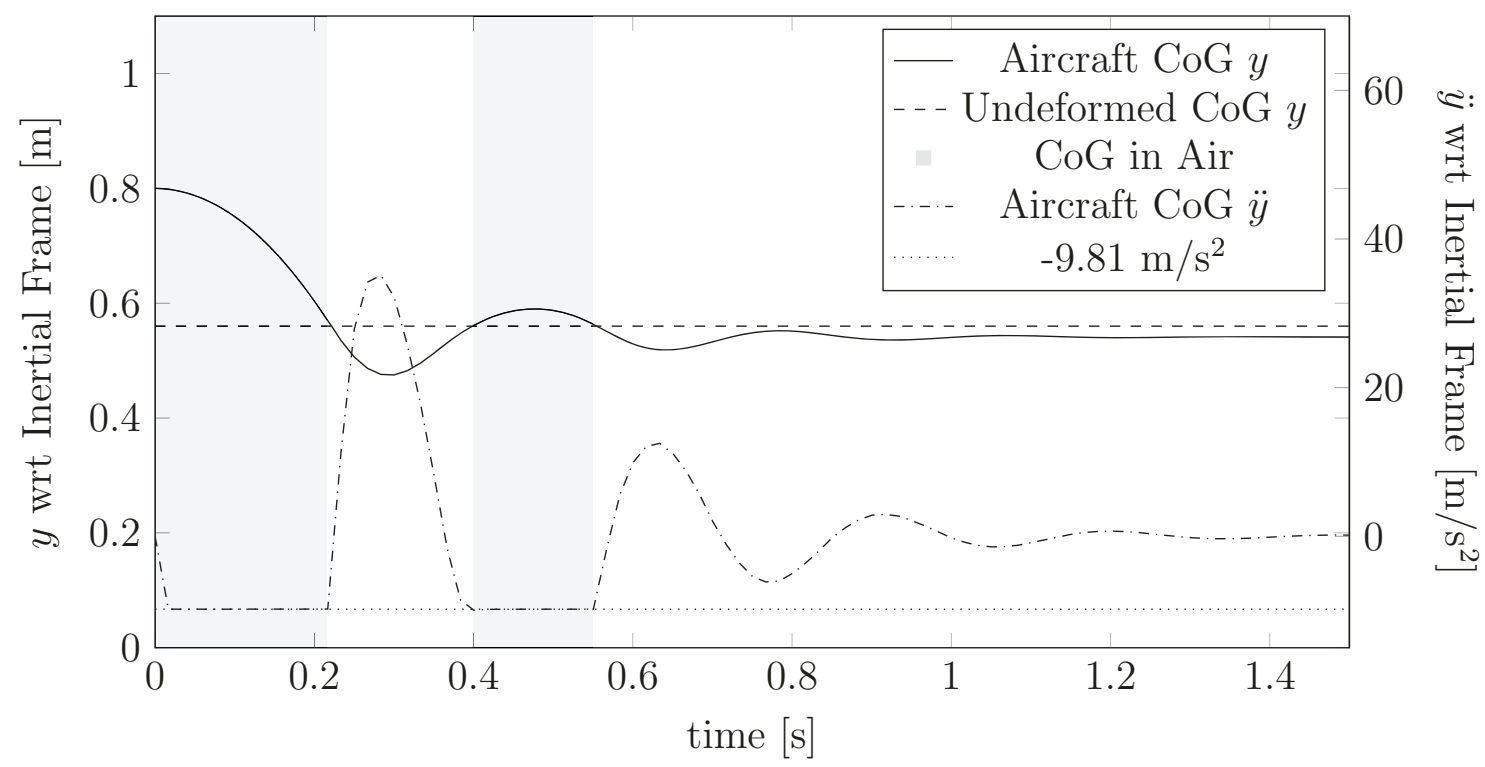

Figure 6.3: Aircraft $\mathrm{CoG}$ y-position and y-acceleration with respect to the inertial frame. The dashed line is the height of the aircraft body CoG resting on a level 0.1 metres thick RSD deck with respect to the inertial frame, with an undeformed landing-gear.

The root-finding function implemented into the ODE solver is also verified to be implemented correctly as the deviations in acceleration occur exactly at times where contact and release from contact occur in the raw generalized solution data. 
Once steady state is achieved as the aircraft rests on the RSD deck, as shown in Table 6.6, the increase in distances of the landing gear feet (in the $\mathrm{x}$ direction) from undeformed to steady-state confirms an outward-splayed stance of the landing gear. The resting height of the CoG at steady-state also decreased which is expected from a splayed landing gear deforming under the weight of the aircraft.

Table 6.6: Case 1 Undeformed versus steady-state helicopter posture

\begin{tabular}{lrr}
\hline DoF & Underformed $[\mathbf{m}]$ & Steady-state $[\mathbf{m}]$ \\
\hline aircraft CoG x & 0.0000 & $8.08 \mathrm{E}-7$ \\
aircraft CoG y & 0.5500 & 0.5411 \\
Left Foot x & -0.3500 & -0.3687 \\
Left Foot y & 0.1100 & 0.1098 \\
Right Foot x & 0.3500 & 0.3687 \\
Right Foot y & 0.1100 & 0.1098 \\
\hline
\end{tabular}

Case 1 verifies that the gravitational model from Equations 4.108 and 4.109 is implemented correctly such that a free-falling aircraft with no air-resistance falls downwards (with respect to the inertial frame) with an acceleration equal to the gravitational acceleration constant $g$. The test case also indicates proper detection of node contact with the RSD deck polygon, and appropriately-oriented upwards normal forces to maintain impenetrability of the polygon.

\subsubsection{Case 2: External Damper Model}

With Case 1 having verified contact response with the RSD deck, more complex test cases can be performed. The discrete external dampers play an important role during the aircraft's touch-down phase. It is expected that variation in damper constants $K_{d}$ and $C_{d}$ will generate different transient responses during touchdown. For Case 2, the aerodynamic models and contact friction model remain turned off to isolate the aircraft collision response from the external dampers. Figures 6.4 through 6.6 show the transient response of the airframe and contact nodes as the stiffness and damping parameters of the external dampers are varied. The parameters used for Case 2 are presented in Table 6.7.

As expected, in Figure 6.4 the higher stiffness coefficient $K_{d} \uparrow$ generates a contact response with larger magnitudes in the oscillations during contact for all bodies and 
Table 6.7: Case 2 Discrete damper parameter variations

\begin{tabular}{lr}
\hline Parameter & Value \\
\hline$K_{d} \uparrow$ & $40000 \mathrm{~N} / \mathrm{m}$ \\
$K_{d} \downarrow$ & $25000 \mathrm{~N} / \mathrm{m}$ \\
$C_{d} \uparrow$ & $500 \mathrm{Ns} / \mathrm{m}$ \\
$C_{d} \downarrow$ & $200 \mathrm{Ns} / \mathrm{m}$ \\
\hline
\end{tabular}

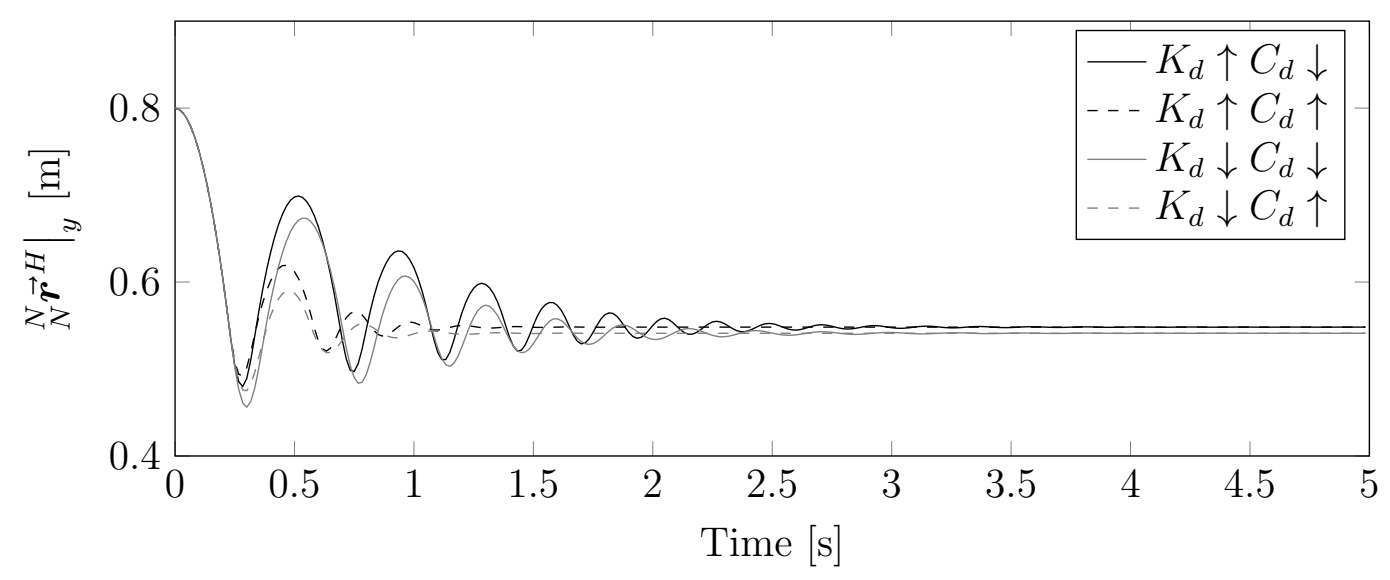

Figure 6.4: Case 2 Airframe vertical response due to discrete external linear damper property variation.

for higher damping coefficient $C_{d} \uparrow$ steady-state was achieved faster due to greater damping response to collision. Further, the steady-state height of the airframe CoG in Figure 6.4 is lower for $K_{d} \downarrow$ which agrees with the notion that with a lower stiffness coefficient, the external damper, and therefore the landing gear, must deflect more to generate an equal amount of force.

Overall, the variation in stiffness and damping parameters show the expected responses. As observed in Figure 6.4, the variation in the damping coefficient affects the damping ratio from apparent log decrements, and the variation of the stiffness coefficient affects the frequency of the oscillations.

The touch-down responses presented in Case 2 verify that the linear external dampers behave as expected when their stiffness and damping parameters are modified, and the symmetrical response at the contact nodes in Figure 6.6 indicates that the general formulas for the external damper forces (Equations 4.106 and 4.103) are implemented correctly for arbitrary external damper configuration. 


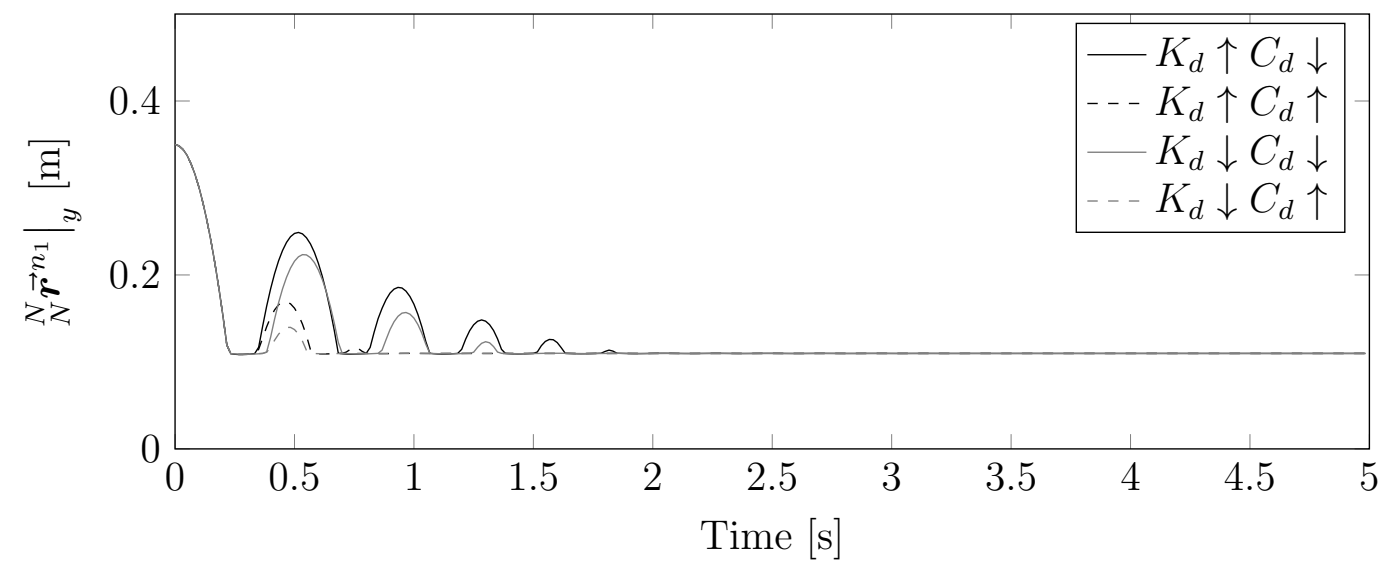

Figure 6.5: Case 2 Landing gear vertical response due to discrete external linear damper property variation.

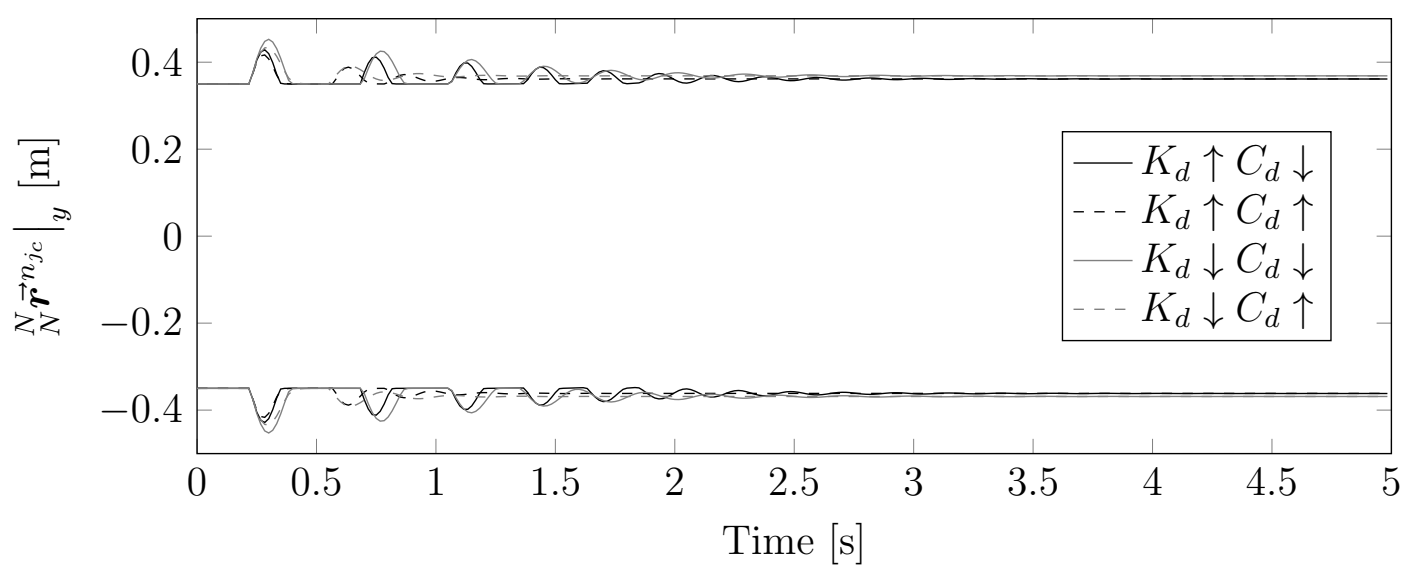

Figure 6.6: Landing gear horizontal response due to discrete external linear damper property variation. Upper data set corresponds to the right landing gear foot $n_{1}$, while the lower data set corresponds to the left landing gear foot $n_{6}$. 


\subsubsection{Case 3: Aircraft Centre of Gravity}

The previous test cases verified level aircraft response for an airframe with a CoG centered between the two landing gear legs. For Case 3, the airframe CoG is horizontally shifted to verify steady-state roll posture, and static equilibrium for asymmetrical loading at the landing gear feet. The aerodynamic models and contact friction model remain turned off for Case 3 .

Figure 6.7 shows the transient roll angle response of the airframe for each CoG position as the aircraft settles on the RSD. Figure 6.8 presents the steady-state roll posture of the airframe as a function of the horizontal shift in position of the airframe CoG.

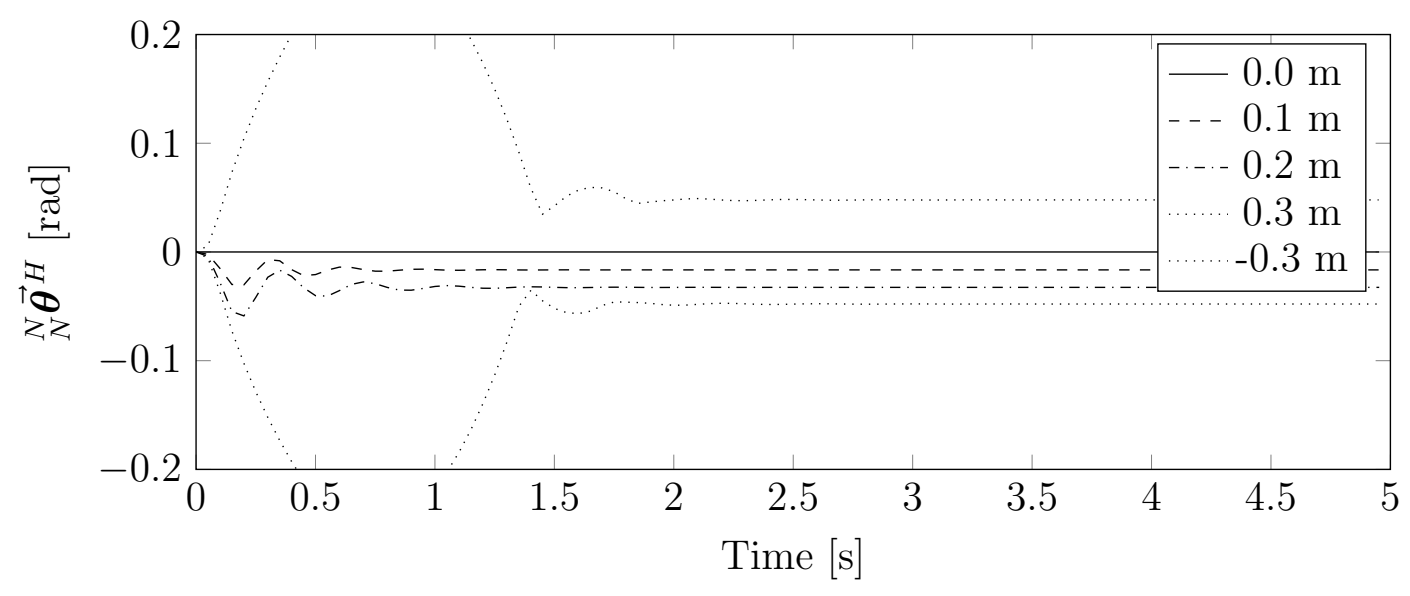

Figure 6.7: Airframe roll-angle response to shifted CoGs. Significant initial roll angles in the $+/-0.3 \mathrm{~m}$ offsets result from the aircraft rolling onto a single landing gear foot upon initial contact with the ground.

The results from Figures 6.7 and 6.8 show that the airframe roll angle at steady-state increases as the airframe CoG is shifted. This verifies the expected behavior for the generic aircraft. As the CoG is shifted further over one side of the hinged landing gear, that landing gear leg assumes more of the load which increases damper compression and lowers that side of the aircraft, resulting in airframe roll. Notably in Figure 6.8, the roll angles at $\pm 0.3 \mathrm{~m}$ are identical, further verifying the implementation of the damper model and hinged airframe interface.

Preliminary validation of the normal forces against basic statics theory is also feasible. Data presented in Figure 6.9 shows that static equilibrium in the vertical direction in inertial frame is satisfied. Airframe and landing gear weights are calculated 


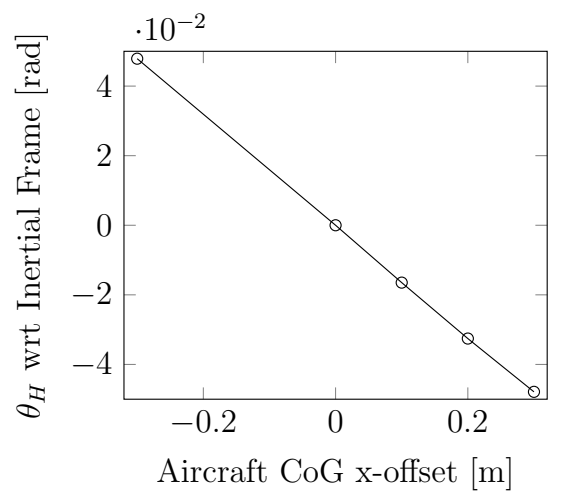

\begin{tabular}{cc}
\hline x-offset $[\mathrm{m}]$ & $\theta_{H}$ Roll [rad] \\
\hline-0.3000 & 0.0479 \\
0.0000 & $5.74 \mathrm{E}-10$ \\
0.1000 & -0.0165 \\
0.2000 & -0.0326 \\
0.3000 & -0.0479 \\
\hline
\end{tabular}

(b) Tabulated Data

(a) Plotted Data

Figure 6.8: Case 3 Steady-state aircraft roll versus airframe CoG x-offset.

from Equations 4.108 and 4.109 transformed to the inertial frame.

In summary, Case 3 verifies the effects of airframe CoG offset on the posture of the aircraft. The airframe rolls in the expected direction as the CoG is shifted. Case 3 also validates the gravitational model and the contact normal forces model under static equilibrium conditions. 


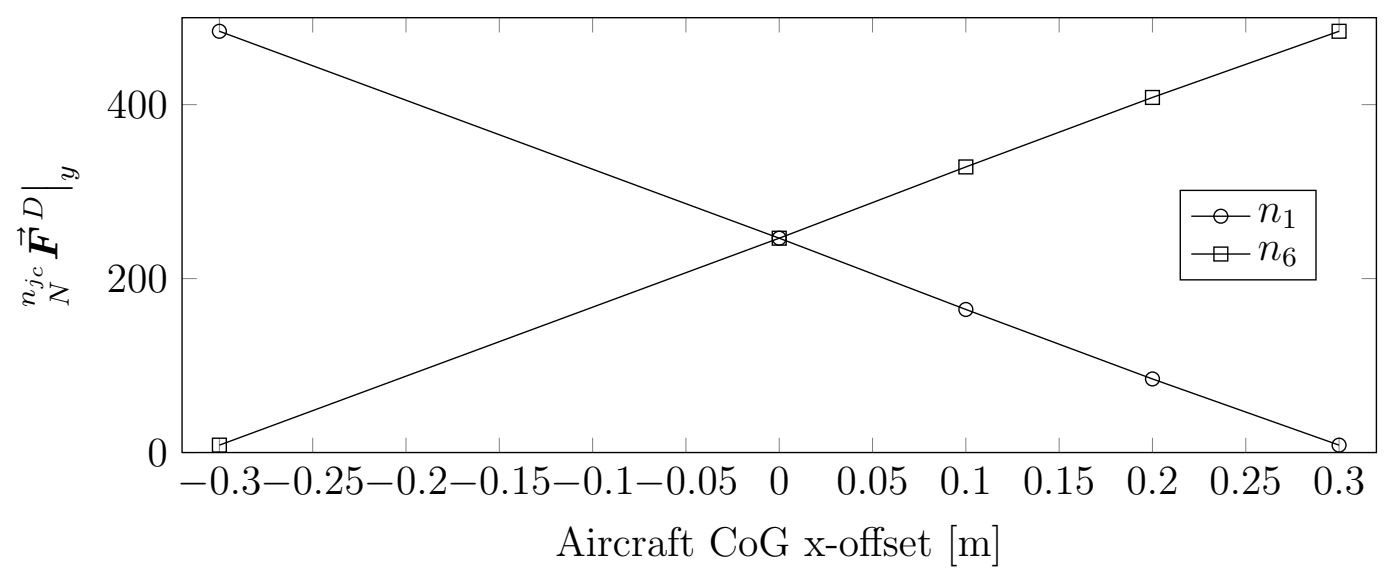

(a) Plotted data

\begin{tabular}{l|rrrrrr}
\hline CoG Offset [m] & & -0.3 & 0.0 & 0.1 & 0.2 & 0.3 \\
\hline$\left.{ }_{N} \vec{F}^{D}\right|_{y}[\mathbf{N}]$ & + & 485.71 & 246.42 & 164.05 & 83.71 & 7.13 \\
$\left.n_{6} \vec{F}^{D}\right|_{y}[\mathbf{N}]$ & + & 7.13 & 246.42 & 328.79 & 409.13 & 485.71 \\
Airframe [N] & - & 490.50 & 490.50 & 490.50 & 490.50 & 490.50 \\
L.G. $[\mathbf{N}]$ & - & 2.34 & 2.34 & 2.34 & 2.34 & 2.34 \\
\hline$\sum F_{y}[\mathbf{N}]$ & $=$ & 0.00 & 0.00 & 0.00 & 0.00 & 0.00 \\
\hline
\end{tabular}

(b) Tabulated Data

Figure 6.9: Case 3 Contact node normal forces versus airframe CoG offset.

\subsubsection{Case 4: Landing Gear DFE Model}

This test case verifies the implementation of the DFE model for fixed and hinged landing gear. The sub-cases for each interface configuration are composed of four level-drop simulations from $0.8 \mathrm{~m}$, where contact friction, and aerodynamic models are turned off. For these sub-cases, the DFE model parameters are modified to exaggerate the behavior of the landing gear during contact. Modified parameters are presented in Table 6.8.

Between each drop test the DFE mesh is also progressively refined. The generic aircraft's landing gear DFE mesh has 6-node, 10-node, 14-node, and 18-node configurations as shown in Figure 6.10. For the hinged configurations, the external dampers are attached to the middle node on each side. 
Table 6.8: Modified DFE model parameters for use in Case 4

\begin{tabular}{lcc}
\hline Parameter & Variable & Value \\
\hline Element young's modulus & $E$ & $6.9 \times 10^{10} \mathrm{~Pa}$ \\
Element second moment of area & $I$ & $5.44 \times 10^{-9} \mathrm{~m}^{4}$ \\
Element density & $\rho$ & $2710 \mathrm{~kg} / \mathrm{m}^{3}$ \\
Element cross section & $A$ & $3.02 \times 10^{-4} \mathrm{~m}^{2}$ \\
Rayleigh damping lower frequency & $\omega_{1}$ & $0 \mathrm{rad} / \mathrm{s}$ \\
Rayleigh damping lower damping Ratio & $\xi_{1}$ & 0.05 \\
Rayleigh damping upper Frequency & $\omega_{2}$ & mesh dependent \\
Rayleigh damping upper damping ratio & $\xi_{2}$ & 0.10 \\
Skid-tube cross-section radius & & $0.01 \mathrm{~m}$ \\
\hline
\end{tabular}

\section{Case 4.1 Fixed interface}

Figures 6.11 plots the transient vertical behavior of the airframe and left contact node during the drop tests for the fixed landing gear. The right foot contact node has the same magnitude of values as the left foot. The plots show that there is energy dissipation in the system between each impact with the RSD deck. This verifies that the proportional damping matrix $[\boldsymbol{C}]$ is working as intended.

Between all DFE meshes, it is difficult to determine if the results converge as is expected for h-method (mesh density) refinement. Yet, if only the 6-, 10-, and 18-node meshes are considered, Figure 6.11 indicates the beginning of convergence in results as the mesh density increases. This is most probably due to the alignment of the common nodes between these DFE meshes as shown in Figure 6.12. The mode shapes of coarser meshes can be expressed as the linear combination of the mode shapes of finer meshes. Moreover, the total structure proportinal damping matrix is derived from the superposition of mode damping. Hence, the observed converging behavior as the DFE model is refined from 6, 10, to 18 nodes. The 14-node model has a different set of eigenvalues due to mesh construction, therefore different mode shapes, and more importantly, slightly different values for $\omega_{1}$ and $\omega_{2}$ as a result. 


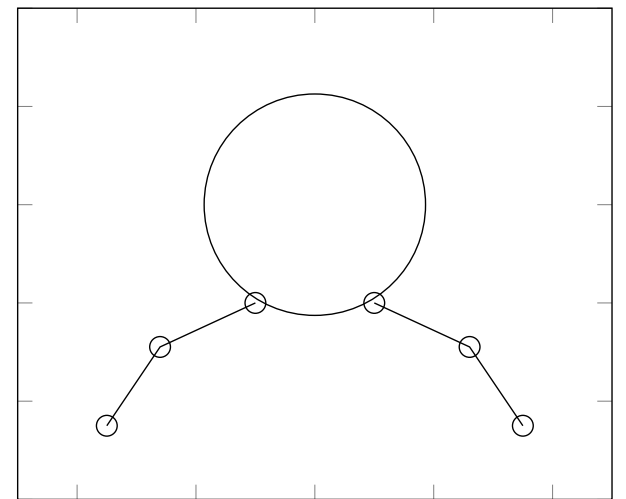

6 nodes, $N_{n}=6$

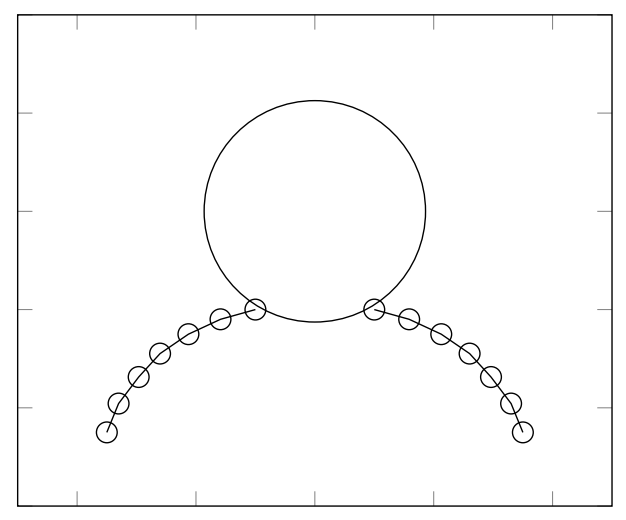

14 nodes, $N_{n}=14$

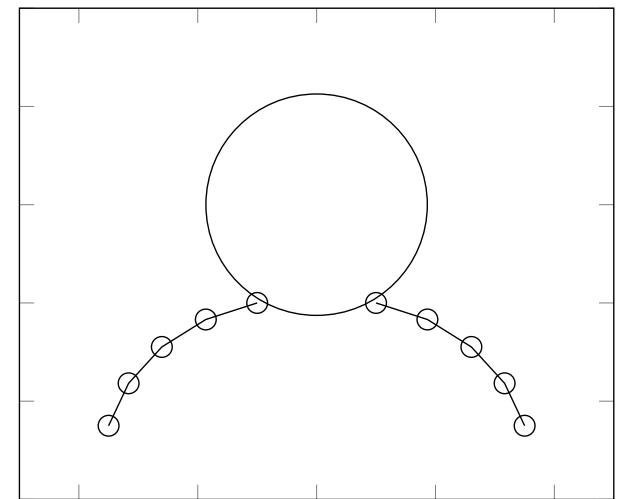

10 nodes, $N_{n}=10$

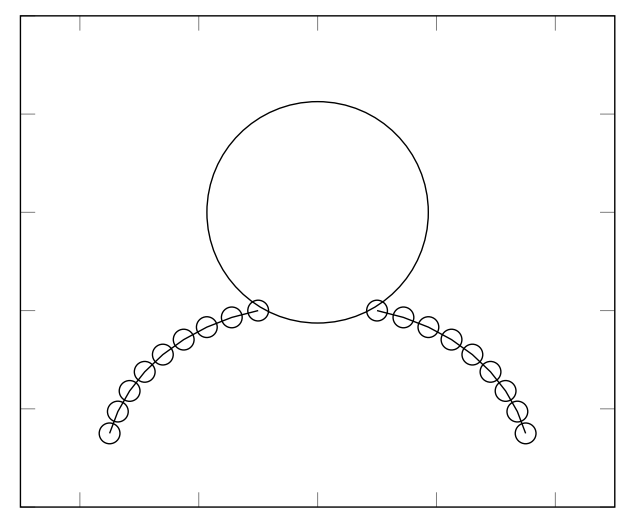

18 nodes, $N_{n}=18$

Figure 6.10: Case 4 DFE model mesh configurations. 

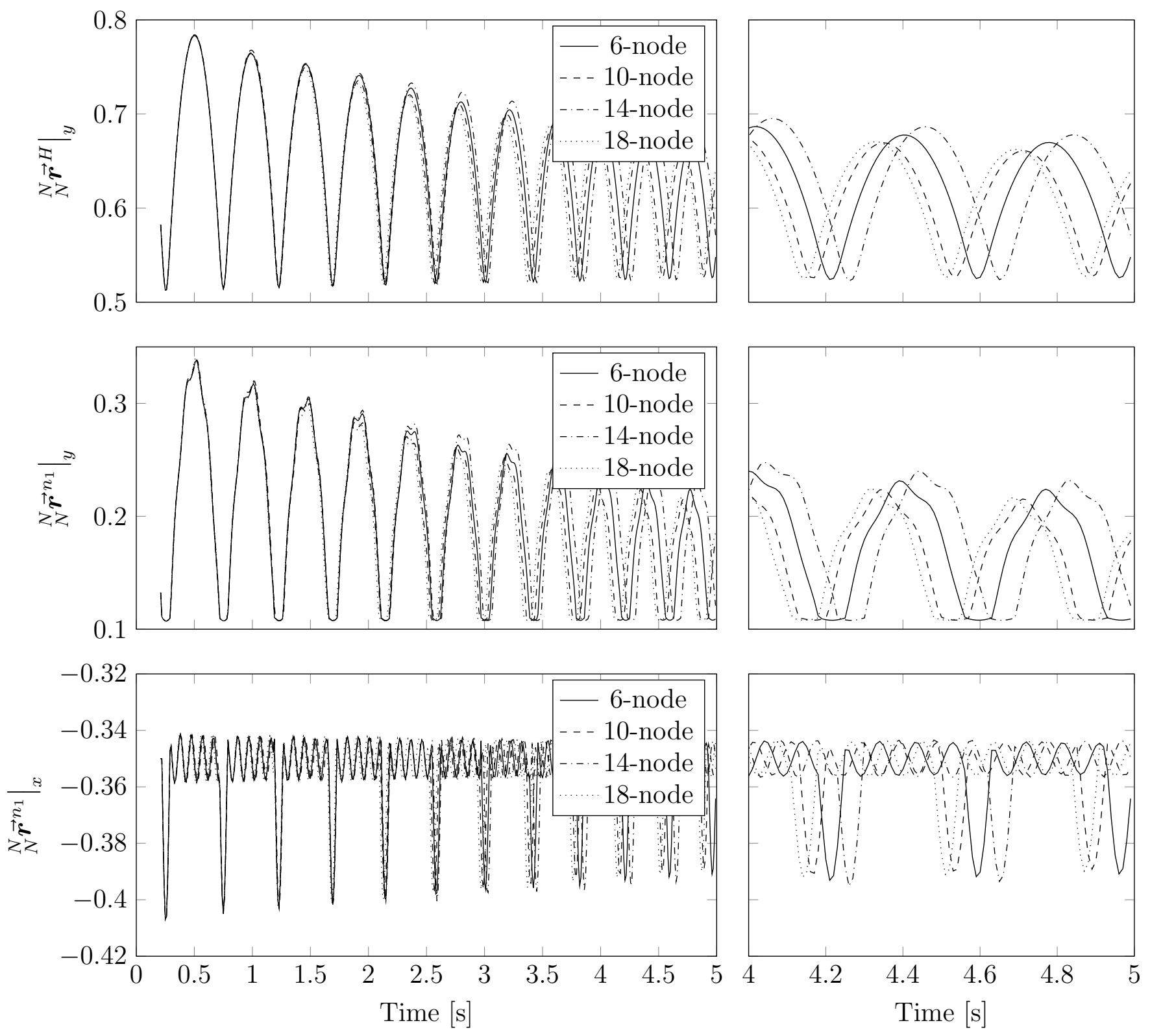

Figure 6.11: Case 4.1 Aircraft response for fixed landing gear with DFE model mesh refinements.

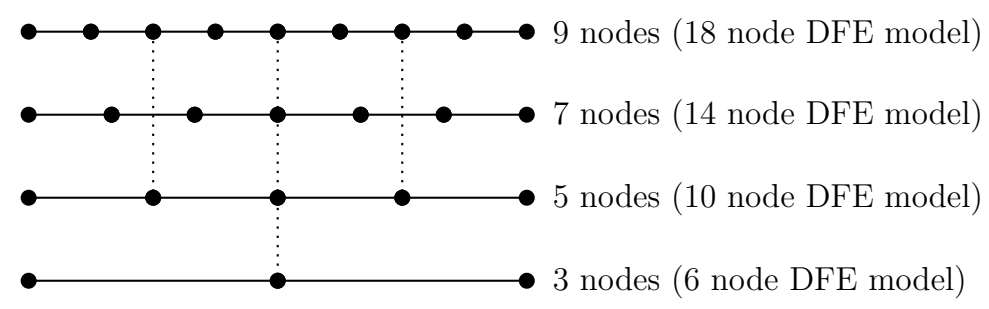

Figure 6.12: Case 4.1 DFE model node construction. 


\section{Case 4.2 Hinged interface}

Figures 6.13 plots the transient vertical behavior of the airframe and left contact node during the drop tests for the hinged landing gear. As the left column of plots show, the energy is dissipated faster through the compression of the external dampers compared to fixed interface landing gear. Due to the hinged interface, the pivoting of the landing gear about this point dominates the deflection in the DFE model. As such, it is not as sensitive to the definition of the DFE mesh as the fixed interface in Sub-case 4.1. Figure 6.13 shows that the results have effectively converged for the 10-, 14-, and 18-node meshes. Therefore, with external dampers, coarser meshes can be used to increase simulation performance.

Sub-cases 4.1 and 4.2 verify the implementation of the DFE model for the skid-type landing gear. First, the aircraft response has verified the correct implementation of Equations 4.90 and 4.91 for fixed and hinged interfaces, and the complete assembly and manipulation of rigid and flexible body dynamics of Section 5.1. Second, the sub-cases have shown that energy is dissipated with each successive impact, which has verified the implementation of a dynamic finite element model which includes damping component. Lastly, the sub-cases have verified the expected convergence through h-method refinement. Though, as Figure 6.11 has shown, fixed-interface landing gear are sensitive to their DFE mesh definitions. 

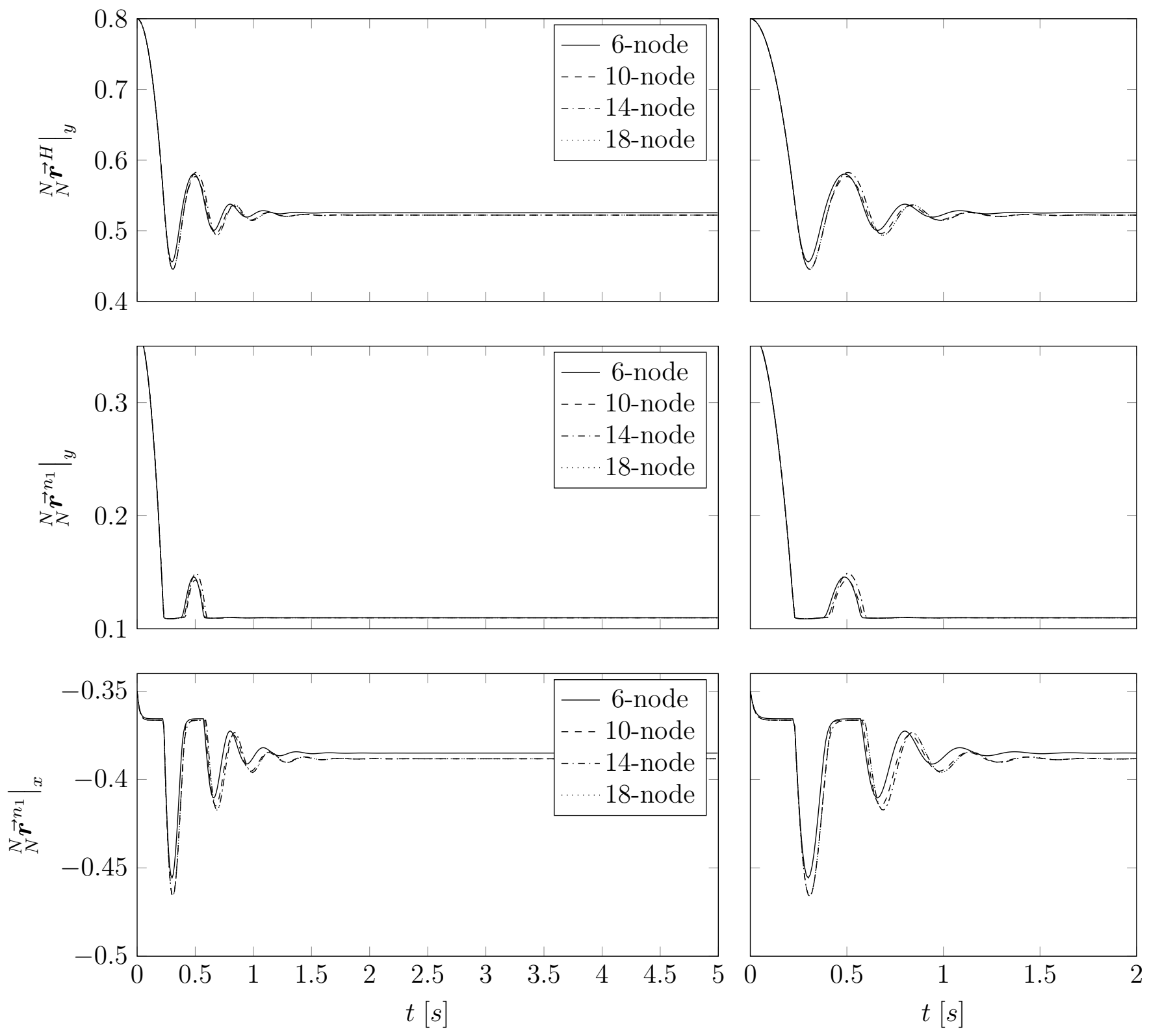

Figure 6.13: Case 4.2 Aircraft response for hinged landing gear with DFE model mesh refinements. 


\subsubsection{Case 5: Contact Dynamics Model}

The contact dynamics model is the core model in SRAMSS. It forms the basis for aircraft-ship interactions and thereby enabling dynamic interface analysis. Case 5 is divided into three sub-cases which independently evaluate normal forces, friction forces, and finally the complete oriented dynamic response.

\section{Case 5.1 Contact Normal Forces}

Similarly to Case 3, Sub-case 5.1 verifies and validates the generation of normal forces under static conditions. Four simulations are performed with configurations as shown in Figure 6.14. For each simulation, the aircraft is dropped onto the ship, and sufficient time is allowed to achieve steady-state rest on deck. Once more, the friction and aerodynamic models are turned off for these simulations.

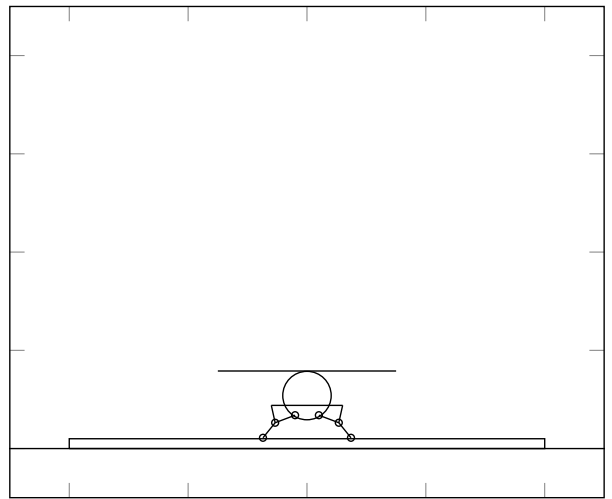

Top Side RSD deck

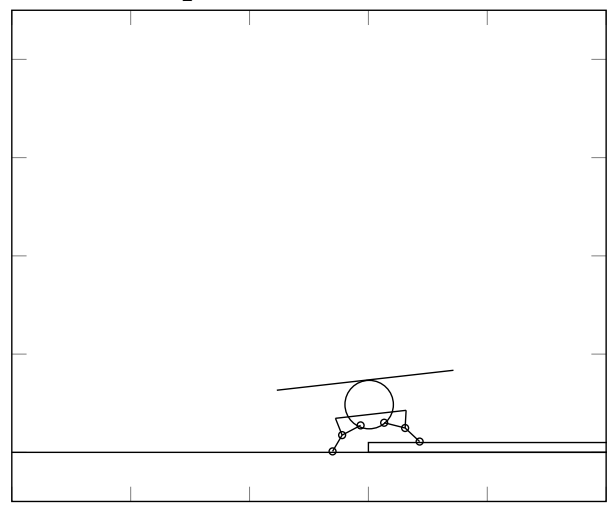

Left Side RSD/Ship deck

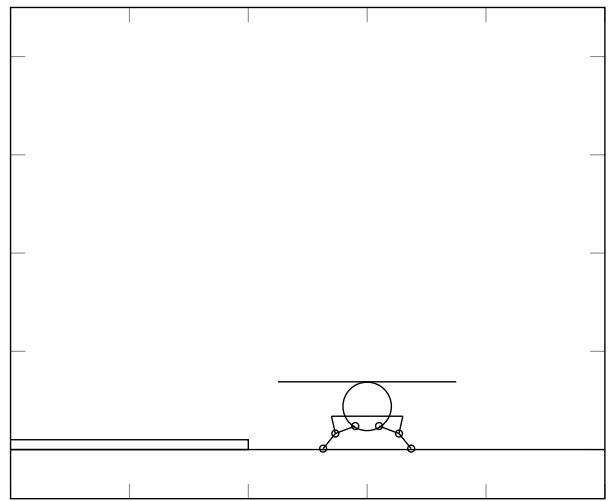

Top Side Ship deck

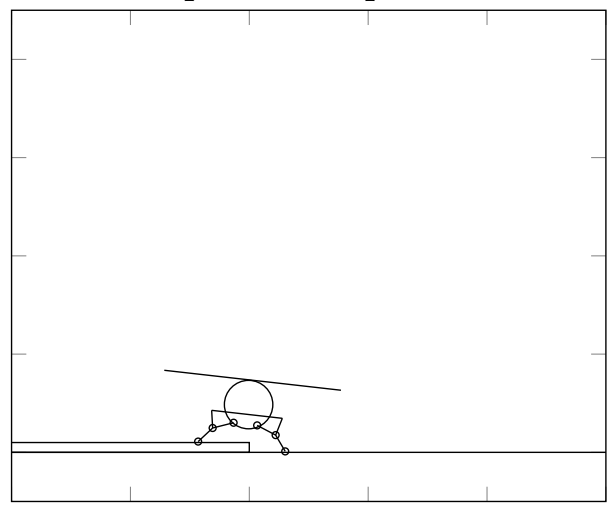

Right Side RSD/Ship deck

Figure 6.14: Case 5.1 Steady-state simulation configurations for normal force evaluation. 
After 5 seconds of elapsed simulated time, the aircraft is considered to be at rest on deck. At this time, the contact normal forces are evaluated. For each simulation, the contact normal forces are listed in Table 6.9. Once more, static equilibrium is validated.

Table 6.9: Case 5.1 Contact normal forces at static equilibrium

\begin{tabular}{l|rrrrr}
\hline Test & & RSD deck & Ship deck & L RSD/ship & R RSD/Ship \\
\hline$\left.n_{1} \overrightarrow{\boldsymbol{F}}^{c}\right|_{y}[\mathbf{N}]$ & + & 246.42 & 246.42 & 280.01 & 212.82 \\
$\left.n_{6} \overrightarrow{\boldsymbol{F}}^{c}\right|_{y}[\mathbf{N}]$ & + & 246.42 & 246.42 & 212.82 & 280.01 \\
Airframe [N] & - & 490.50 & 490.50 & 490.50 & 490.50 \\
L.G. [N] & - & 2.34 & 2.34 & 2.34 & 2.34 \\
\hline$\sum F_{y}[\mathbf{N}]$ & $=$ & 0.00 & 0.00 & 0.00 & 0.00 \\
\hline
\end{tabular}

\section{Case 5.2 Contact Friction Forces}

As part of the oriented dynamic response to contact, a friction force is applied to the landing gear nodes parallel to the contact surface. The flexibility of the contact mechanics algorithm in SRAMSS allows many friction models to be used. Currently, the LuGre model has been implemented. The LuGre model can also be reduced to a simple Coulomb frictional slider model by equating $\mu_{s}=\mu_{d}$.

In the Sub-case 5.2 simulations, for a generic aircraft at rest on deck, the ship's roll angle is increased through the point where the aircraft begins to slide across the ship deck. This case verifies that the generic aircraft begins to slide at a ship roll angle ${ }_{N}^{N} \overrightarrow{\boldsymbol{\theta}}^{S}=\tan ^{-1} \mu$.

For Sub-case 5.2, friction parameters have been modified to capture the desired phenomena before the aircraft's roll-over point is reached. The modified parameters of Table 6.1 are found in Table 6.10.

Given the parameters from Table 6.10, the aircraft is predicted to roll at ${ }_{N}^{N} \overrightarrow{\boldsymbol{\theta}}^{S}=$ -0.3806 radians using the LuGre model, and ${ }_{N}^{N} \overrightarrow{\boldsymbol{\theta}}^{S}=-0.1974$ radians using the Coulomb model.

Figure 6.15 shows the relative sliding velocity of the leading landing gear foot, DFE node $n_{6}$. As expected, the aircraft begins sliding at a larger ship roll angle for the LuGre model since it is able to model static friction. Figure 6.15 also confirms the 
Table 6.10: Modified aircraft simulation parameters for use in Sub-case 5.2

\begin{tabular}{lcc}
\hline Parameter & Variable & Value \\
\hline Friction micro-displacement stiffness & $K_{f}$ & $1 \times 10^{6} \mathrm{~N} / \mathrm{m}$ \\
Friction micro-displacement damping & $C_{f}$ & $1 \times 10^{4} \mathrm{Ns} / \mathrm{m}$ \\
Stribeck velocity threshold & $v_{s}$ & $0.01 \mathrm{~m} / \mathrm{s}$ \\
Dynamic friction coefficient (LuGre) & $\mu_{d}$ & 0.2 \\
Static friction coefficient (LuGre) & $\mu_{s}$ & 0.4 \\
Dynamic friction coefficient (Coulomb) & $\mu_{d}$ & 0.2 \\
Static friction coefficient (Coulomb) & $\mu_{s}$ & 0.2 \\
\hline
\end{tabular}

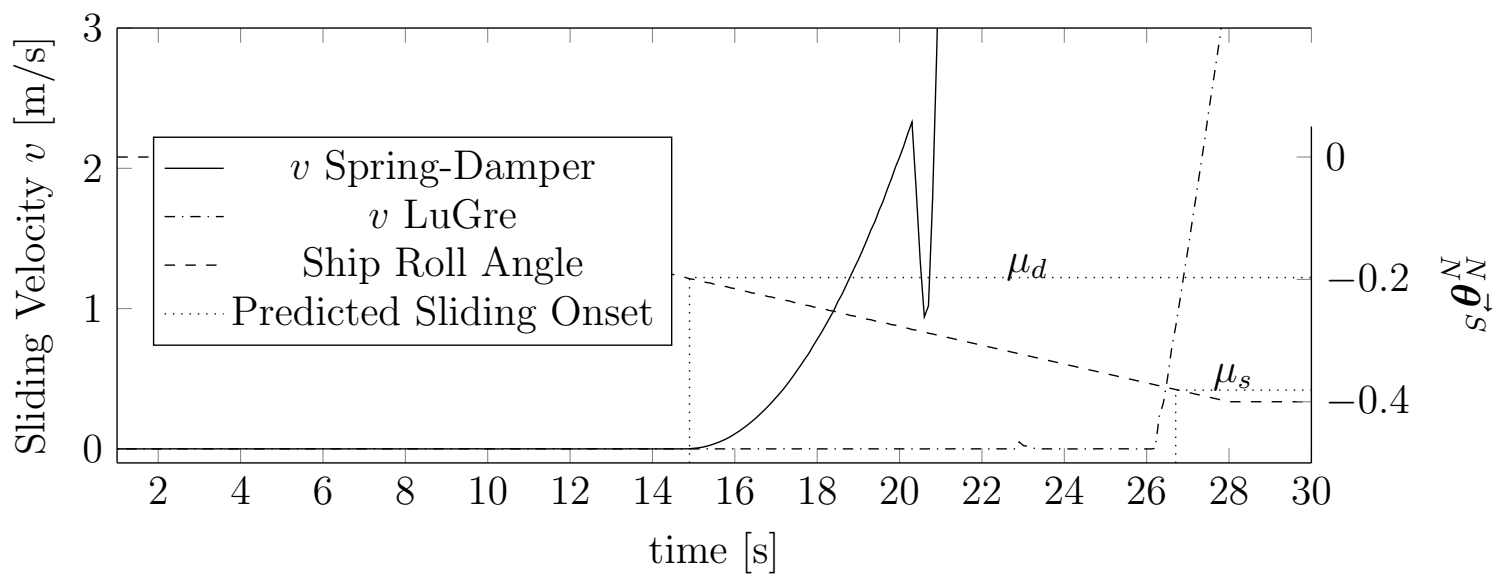

Figure 6.15: Case 5.2 Sliding velocity of leading (right) landing gear foot along the RSD deck as the roll angle of the ship increases.

predicted roll angle -0.1974 radians for the Coulomb model. The drop in Coulomb sliding velocity after 20 seconds is an artifact of the aircraft falling off the ship deck.

However, the LuGre model lets the aircraft slip at -0.3724 radians, earlier than predicted. The trailing foot $\left(n_{1}\right)$ exhibits stick-slip behavior as indicated by the oscillating friction value in Figure 6.16. These oscillations are the result of weight transfer from the trailing to leading foot $\left(n_{6}\right)$ due to a reduction in normal force at the trailing foot. This is apparent from the decreasing values of $F_{d}$ and $F_{s}$ for $n_{1}$, and increase for $n_{6}$. The drop in friction force on $n_{1}$ shifts the aircraft, which increases the friction force generated by the frictional slider of $n_{6}$. Immediately before slipping around 26 seconds, the third weight transfer to $n_{6}$ saturates $F_{L u G r e}$ to $F_{s}$. The maximum frictional slider distance (bristle deflection) $\Delta_{f, \max }$ allowed by Stribeck equation (Eq. 4.49) is achieved. No greater frictional force can be generated to maintain the 

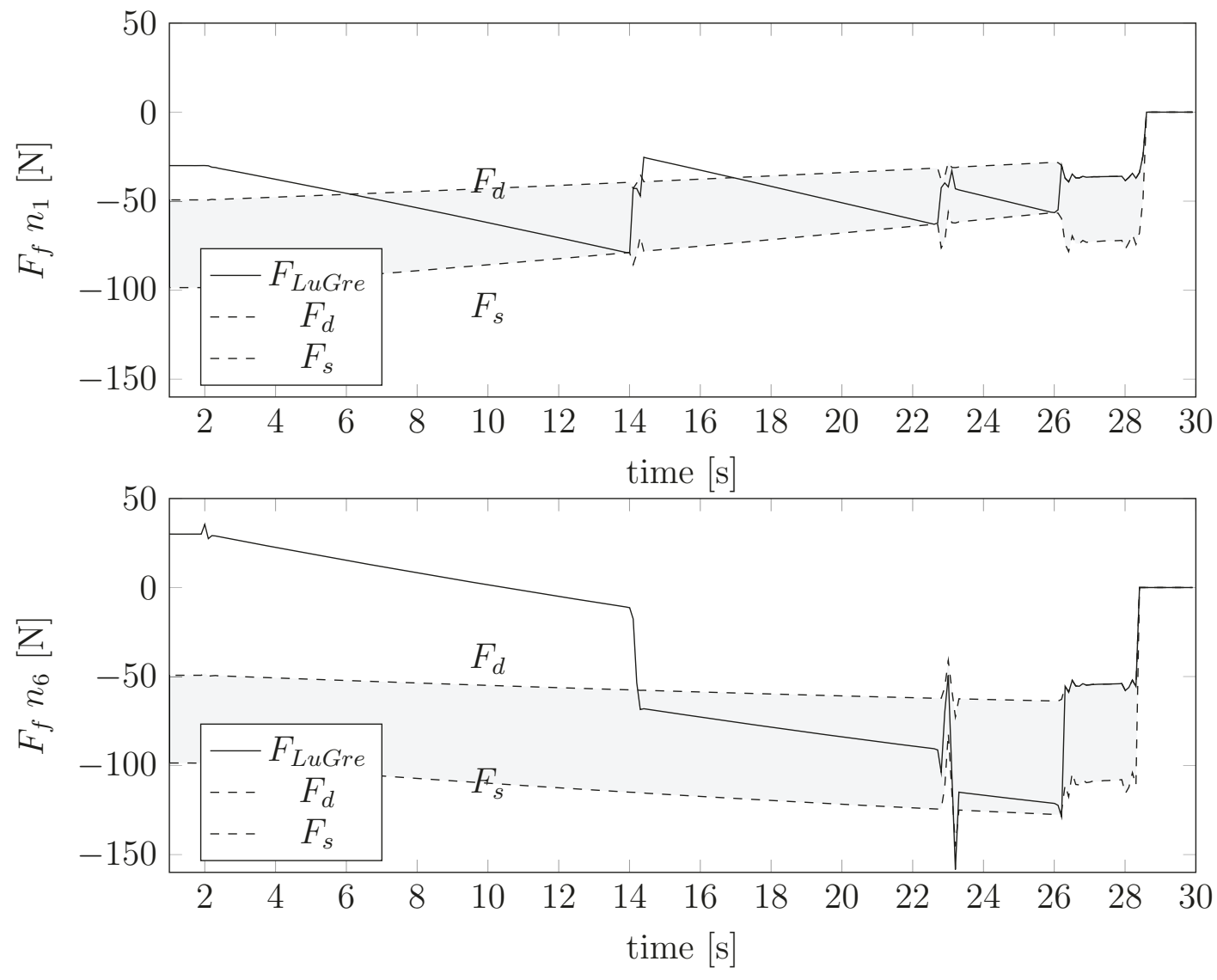

Figure 6.16: Case 5.2 Trailing $\left(n_{1}\right)$ and leading $\left(n_{6}\right)$ landing gear feet frictional forces

airfcraft's position on deck, thus it begins to slide pre-maturely.

While the LuGre model does not exactly verify the prediction, the predicted value does not consider transient phenomena which dynamically varies the upper limit of static friction. In any case, the implementation of the LuGre model can still be verified. Through the Stribeck equation, the maximum friction force is dynamically tuned to exhibit static and dynamic friction phenomena. In Figure $6.16, F_{\text {LuGre }}$ is allowed to grow as required to maintain the aircraft's position. Once $F_{\text {LuGre }}$ saturates to the maximum allowable static friction $F_{s}$, sliding occurs. At the onset of sliding, $F_{\text {LuGre }}$ immediately drops to the dynamic friction value $F_{d}$. This verifies that both static and dynamic friction phenomena of the LuGre model are implemented correctly.

The parameters used for this sub-case are arbitrary, and tuned to visually show the desired phenomena. True parameters can only be determined through extensive experimental testing. Nevertheless, the LuGre model is verified to capture the friction phenomena predicted. 


\section{Case 5.3 Oriented dynamic response}

For Case 5, the generation for normal and friction forces has been verified. Response to contact on surfaces other than the top sides of polygons, and when polygons are prescribed non-zero motion remain to be verified.

Oriented dynamic response is verified through three simulations. Each simulation is configured to involve different interactions with a moving RSD deck. A visual description of the simulations is presented in Figure 6.17.

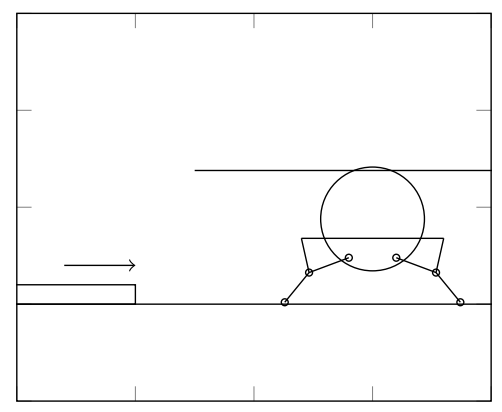

RSD push

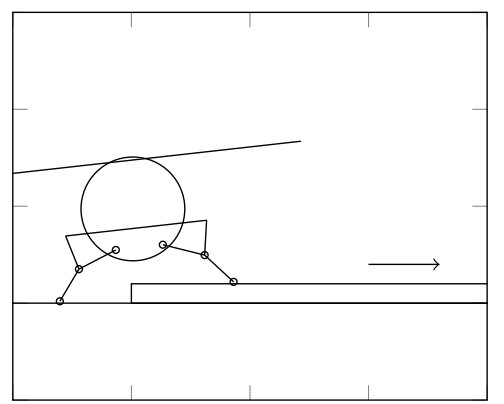

RSD drag

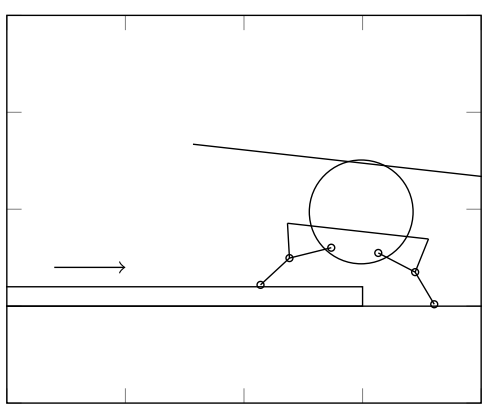

RSD hook

Figure 6.17: Case 5.3 Oriented dynamic response simulation configurations.

For the first simulation, the aircraft starts at rest on the ship deck. The RSD deck is prescribed translational velocity of $0.3 \mathrm{~m} / \mathrm{s}$ starting at 2 seconds. For this simulation, the aircraft's response is predicted to be pushed along the ship deck through appropriately-oriented normal forces from the side of the RSD deck acting at $n_{1}$, .

As shown in Figure 6.18, the aircraft is dropped onto the deck where it comes to rest. At 2 seconds, the RSD deck begins translation. Shortly thereafter, the RSD deck comes into contact with the left foot $\left(n_{1}\right)$. The normal forces generated by the side of the RSD deck overcome the friction forces of the ship deck acting at the contact nodes which allows the RSD deck to push the aircraft along. The 'push' is evidenced by the overlapping 'RSD Right Surface' and $\left.{ }_{N}^{N} \overrightarrow{\boldsymbol{r}}^{n_{1}}\right|_{x}$ plot lines. At 9.4 seconds, the aircraft begins to fall off the ship deck, indicated by the large drop in $y$-position in Figure 6.18. This simulation verifies the physically-expected response of the aircraft.

The second simulation has the aircraft at rest with the left foot $\left(n_{1}\right)$ on the ship deck and right foot $\left(n_{6}\right)$ on the RSD deck. Again, the RSD deck is prescribed translational velocity of $0.3 \mathrm{~m} / \mathrm{s}$ starting at 2 seconds. Here, it is expected that the aircraft's response is to be dragged along by friction forces until the aircraft has slipped off the 


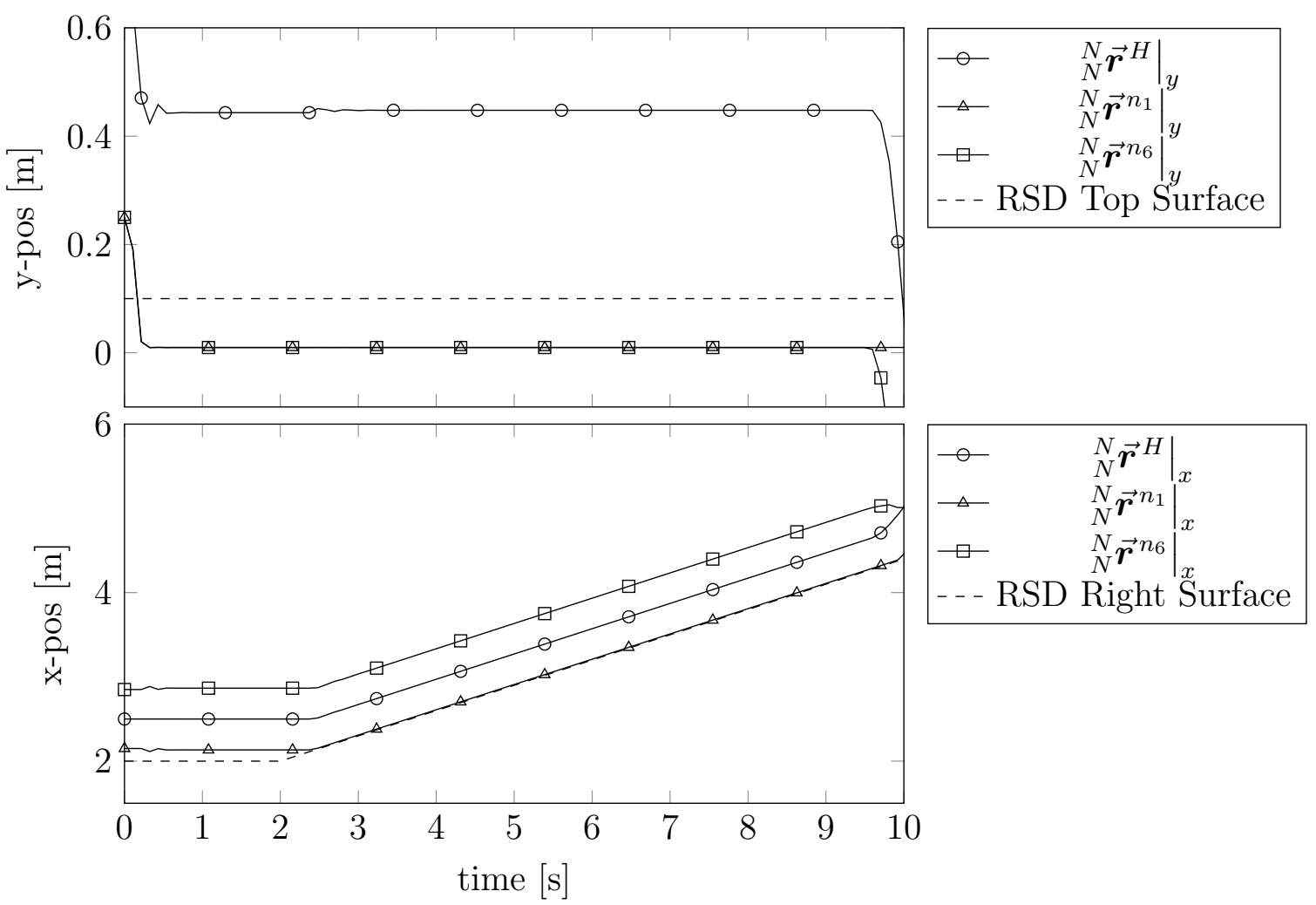

Figure 6.18: Case 5.3 Position of airframe and contact nodes in the 'RSD push' scenario.

RSD deck. The slipping is predicted by the weight shift over the left foot, the nodal forces in Table 6.9 support this prediction.

The aircraft response prediction is verified in Figure 6.19. As the RSD deck starts moving, the posture of the landing gear is expanded by friction forces until equilibrium is reached through the external dampers. The drop in $y$-position for the airframe and $n_{6}$ just before 4 seconds indicates that the aircraft has slipped off the RSD deck onto the ship deck. From 2 seconds until the drop off the RSD deck, the translational motion of the RSD deck drags the aircraft along. Due to the higher normal forces on $n_{1}$, and the relative sliding velocity between $n_{6}$ and the RSD deck, the friction forces on $n_{1}$ are greater. The larger friction force at $n_{1}$ allows the aircraft to slide along the ship deck, but not at the same velocity as the translation of the RSD deck. This difference in translational velocities causes $n_{6}$ to eventually fall off the RSD deck as previously stated.

For the third simulation of Sub-case 5.3, the aircraft is at rest with the left foot $\left(n_{6}\right)$ on the ship deck and right foot $\left(n_{1}\right)$ on the RSD deck. Again, the RSD deck 


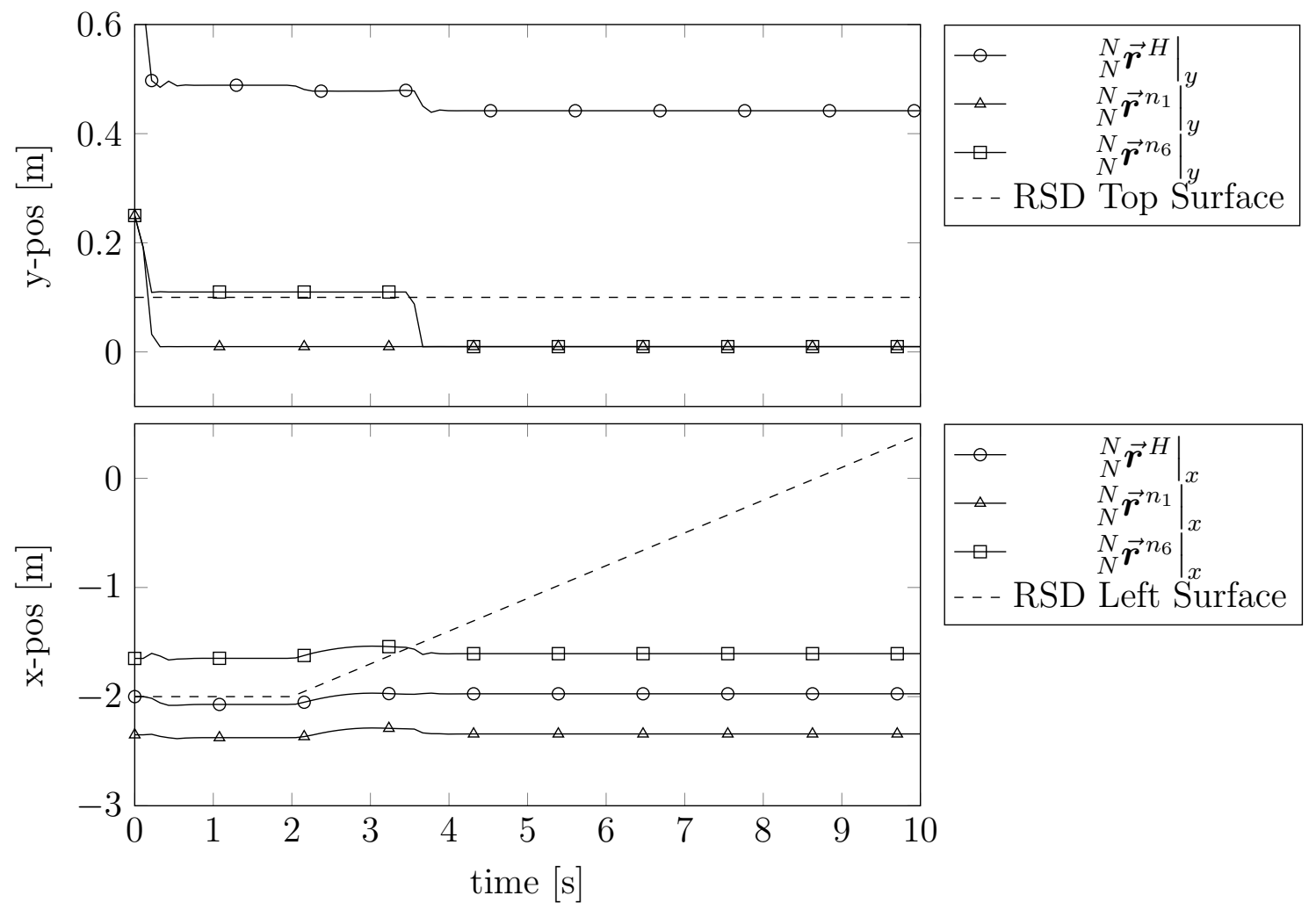

Figure 6.19: Case 5.3 Position of airframe and contact nodes in the 'RSD drag' scenario.

is prescribed translational velocity of $0.3 \mathrm{~m} / \mathrm{s}$ starting at 2 seconds. Opposite to the second case, the predicted response is such that $n_{1}$ slides atop the RSD deck until the side of the RSD deck comes into contact with $n_{6}$. At this point, $n_{6}$ is hooked into the corner formed by the ship deck and side of the RSD deck. This 'hook' will drag the aircraft along at the same rate as the prescribed translational motion of the RSD deck.

Figure 6.20 shows the aircraft coming to rest with the left foot $\left(n_{6}\right)$ on the ship deck and right foot $\left(n_{1}\right)$ on the RSD deck. At the onset of RSD deck translation, the aircraft exhibits a slight translational motion due to the difference in frictional forces. Around 3 seconds, the right side of the RSD deck comes into contact with $n_{6}$ resting on the ship deck, the 'hook', and begins to drag the aircraft along. The 'hook' is evidenced by the overlapping 'RSD Right Surface' and $\left.{ }_{N}^{N} \overrightarrow{\boldsymbol{r}}^{n_{6}}\right|_{x}$ plot lines. The aircraft response expressed in this simulation verifies the predictions made.

Through the simulations performed in Sub-cases 5.1, 5.2, and 5.3, the contact dynamics model is verified to be implemented correctly. Regardless of the contact 


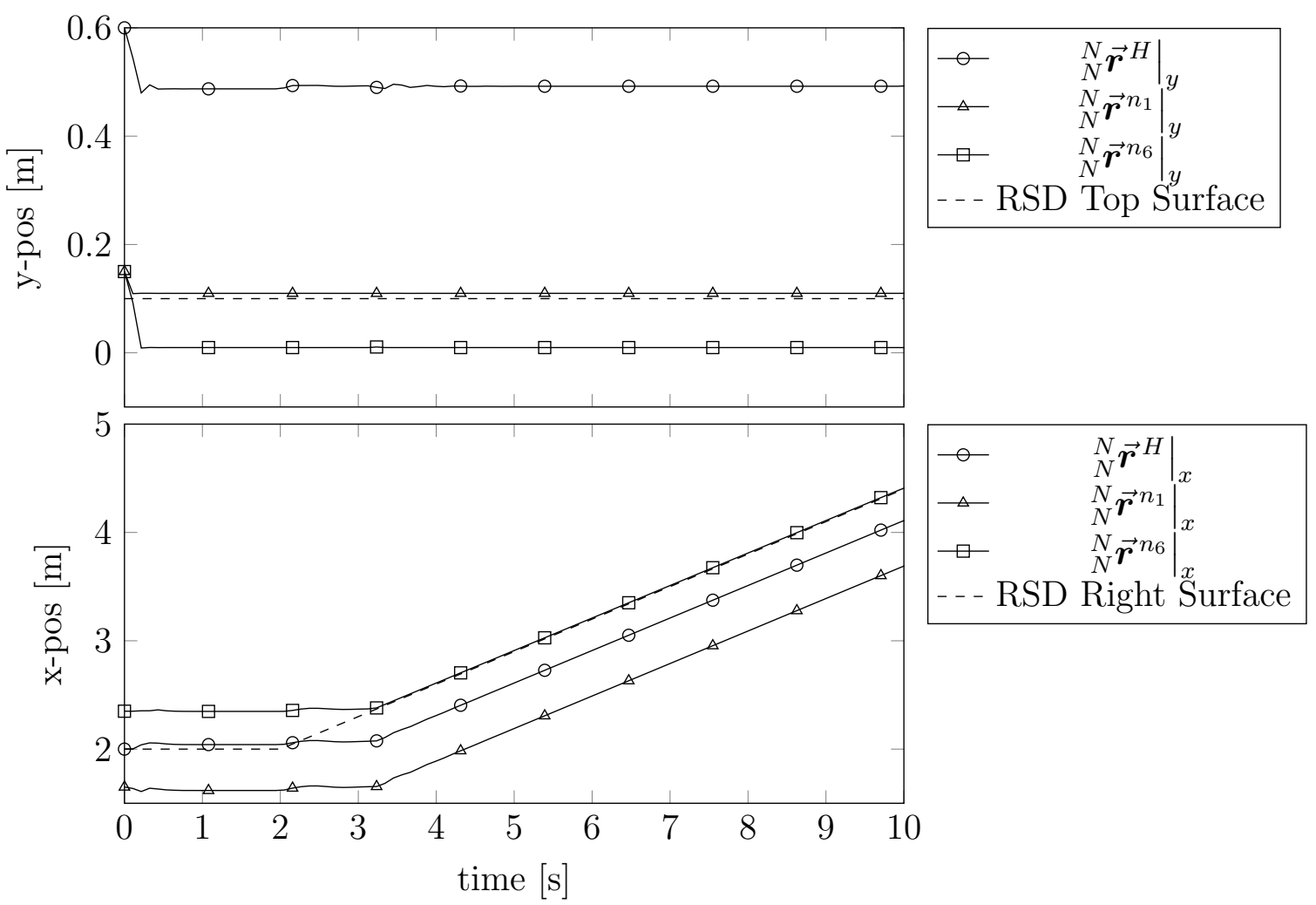

Figure 6.20: Case 5.3 Position of airframe and contact nodes in the 'RSD hook' scenario.

surface, the normal and friction forces are appropriately-oriented such that predictable aircraft response is observed in the simulations.

\subsubsection{Case 6: Aerodynamics Models}

Case 6 focuses on the verification of the aerodynamic body drag models, and BEM model for rotor thrust. This case is divided into four sub-cases verifying the aerodynamic drag model; rotor thrust without wind; rotor thrust with cross-wind; and rotor thrust with cross-wind and cyclic setting compensation. Additionally, there is preliminary validation of the BEM model against published CFD data.

\section{Case 6.1 Aerodynamic Body Drag Model}

In this sub-case, implementation of the aerodynamic model is verified. The aerodynamic body drag model, presented in Equations 4.58 through 4.64, is developed in three dimensions. For the planar verification of the model, drag forces induced by 
relative wind velocity in the $N$ frame $x$ and $y$ directions are evaluated.

For the $x$-direction, cross-winds are applied to the aircraft at rest on the RSD deck. For this test, the friction model is turned off to isolate the effects of aerodynamic drag. Between 1.0 and 1.5 seconds in simulation time, the cross-wind velocity is linearly increased from $0 \mathrm{~m} / \mathrm{s}$ to the prescribed value.

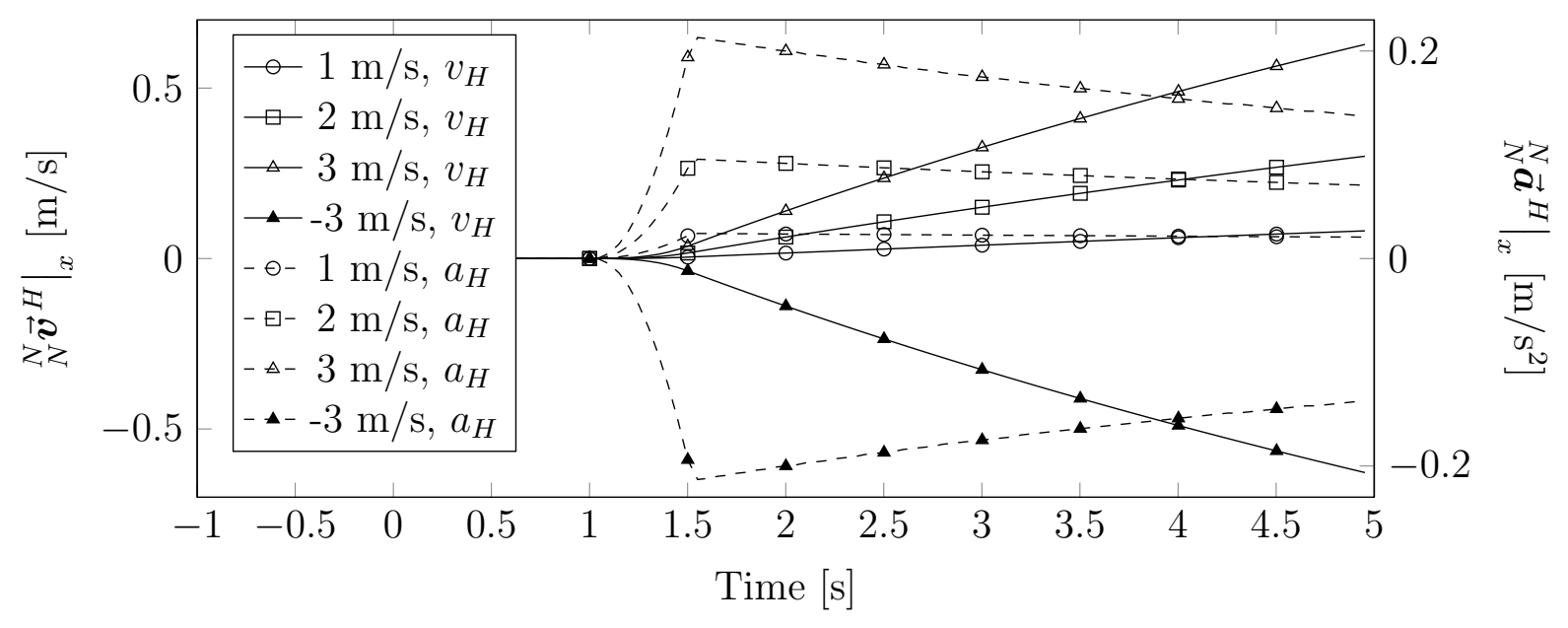

Figure 6.21: Case 6.1 Aircraft horizontal sliding velocity due to induced drag.

The sliding velocity and acceleration of the aircraft under different cross-wind velocity conditions is presented in Figure 6.21. As expected, an increase in the wind velocity increases the rate of sliding velocity of the aircraft. The aircraft exhibits large initial accelerations at the onset of sliding. As the aircraft's sliding velocity increases, its acceleration decreases since the relative wind speed with respect to the $\mathrm{CP}$ is decreasing.

A simple force balance verifies that the aerodynamic drag formula in Equation 4.63 is implemented correctly. For a cross-wind speed of $3 \mathrm{~m} / \mathrm{s}$ at simulated time of $3 \mathrm{sec}-$ onds and total aircraft mass of $50.2383 \mathrm{~kg}$, the aircraft's sliding velocity is $0.3262 \mathrm{~m} / \mathrm{s}$ with an acceleration of $0.1745 \mathrm{~m} / \mathrm{s}^{2}$.

$$
\sum F_{x}=m a_{x}
$$

where

$$
\begin{aligned}
m a_{x} & =\left(m_{H}+[M]_{x}\right) a_{x}=(50.2383 \mathrm{~kg})\left(0.1745 \mathrm{~m} / \mathrm{s}^{2}\right)=8.7666 \mathrm{~N} \\
\sum F_{x} & =\frac{1}{2} \rho \boldsymbol{A}_{x}\left(\left.{ }_{H}^{C P} \overrightarrow{\boldsymbol{v}}^{W}\right|_{x}\right)^{2} \\
& =(0.5)\left(1.225 \mathrm{~kg} / \mathrm{m}^{3}\right)\left(2 \mathrm{~m}^{2}\right)(3 \mathrm{~m} / \mathrm{s}-0.3262 \mathrm{~m} / \mathrm{s})^{2}=8.7578 \mathrm{~N}
\end{aligned}
$$


Interpolation within raw simulation data to obtain sliding acceleration, and slight aircraft roll due to the ${ }_{H}^{H} \overrightarrow{\boldsymbol{r}} C P$ should account for the error in the calculations above.

In the $y$ direction test, the aircraft is dropped towards the RSD deck from a height of 0.8 metres. Cross-winds are set to $0 \mathrm{~m} / \mathrm{s}$ and the friction model is turned off.

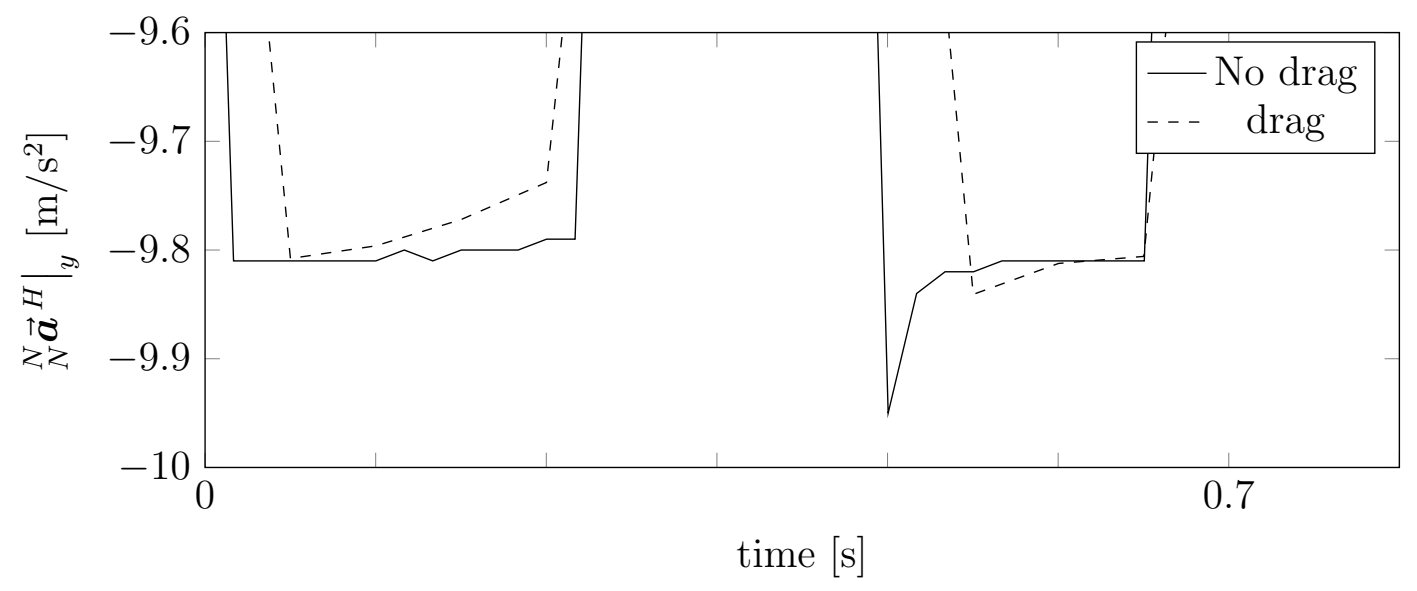

Figure 6.22: Case 6.1 Aircraft vertical acceleration due to induced drag.

Figure 6.22 shows the vertical accelerations of the airframe during drop tests with and without aerodynamic body drag. The drop without drag maintains an acceleration equal to gravitational acceleration, whereas the induced drag reduces the vertical acceleration of the aircraft in the other case.

The magnitude and orientation of drag forces in the simulations of Sub-case 6.1 verify that Equation 4.63 is implemented correctly in SRAMSS.

\section{Case 6.2 Rotor Thrust Without Wind}

Sub-cases 6.2 through 6.4 evaluate the implementation of the BEM model for rotor disc thrust and roll moment generation. In Sub-case 6.2, the rotor thrust is evaluated for an aircraft beginning at rest on the RSD deck without relative wind across the deck. The number of BEM sectors $N_{\psi}$ and rotor blade elements $N_{e}$ is varied between each simulation in Sub-case 6.2. The time-varied parameters for the BEM model are found in Figure B.1 in Appendix B.1. Further, the aerodynamic body drag model is turned off for this test to isolate thrust force and rolling moment acting on the aircraft.

Figure 6.23 shows that rotor disc RPM increase from $0 \mathrm{rad} / \mathrm{s}$ to $135 \mathrm{rad} / \mathrm{s}$ from 2 to 6 seconds increases rotor disc thrust from $0 \mathrm{~N}$ to nearly $600 \mathrm{~N}$. Since there is no relative 


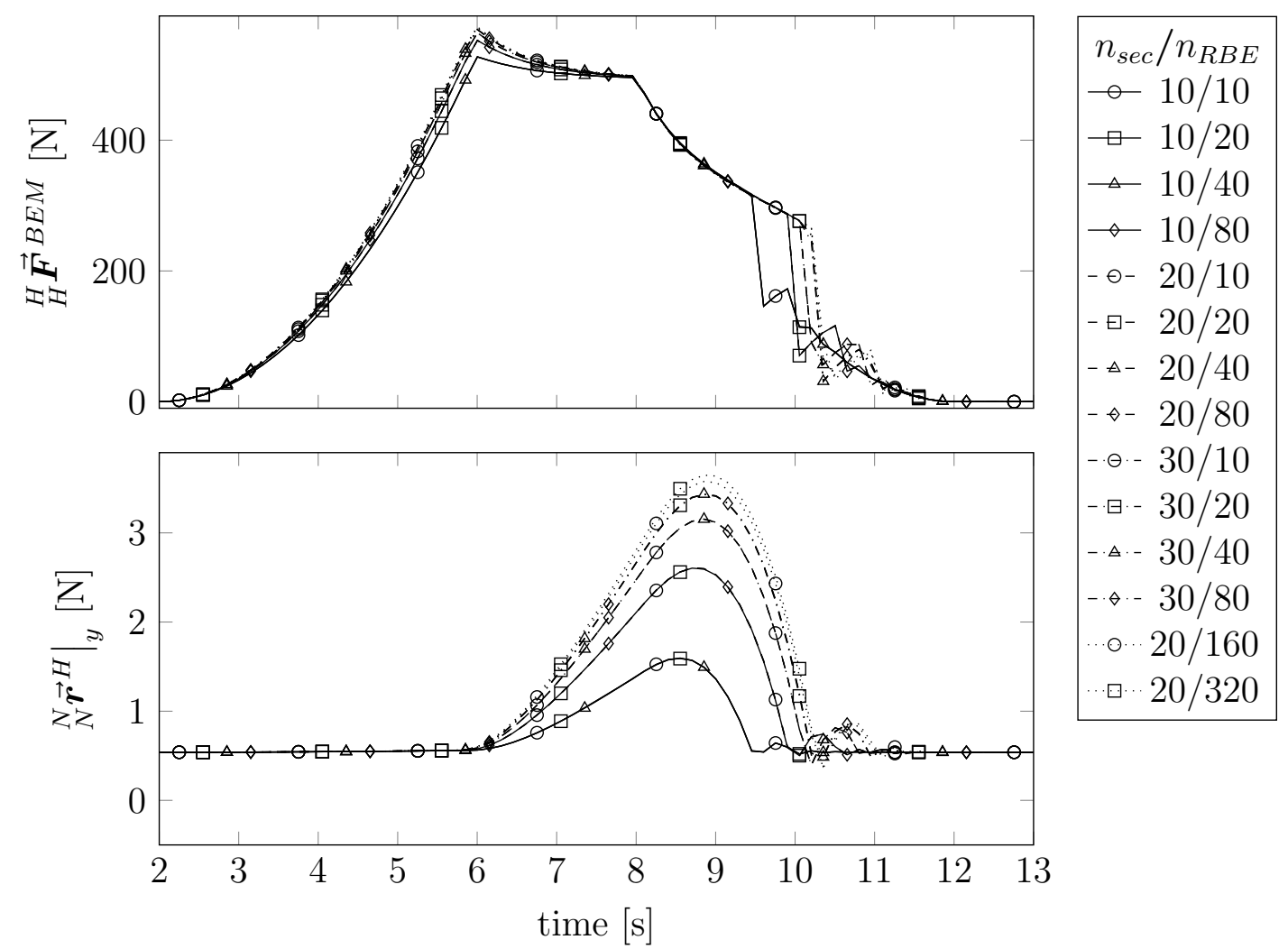

Figure 6.23: Case 6.2 Rotor disc thrust and airframe CoG height above the RSD deck.

wind, the advancing and retreating sides of the rotor disc generate equal amounts of thrust, which results in a vertical ascent by the aircraft beginning at 6 seconds. The magnitude of thrust drops as the aircraft lifts off due to an increasing axial flow through the rotor disc. The RPM is decreased to $0 \mathrm{rad} / \mathrm{s}$ from 8 to 12 seconds which allows the aircraft to touch down on the RSD desk around the 10-second mark. Strictly vertical aircraft motion, and zero rotor disc moment generation verifies uniformity in thrust generated in no relative wind conditions.

Another aspect to verify the correct implementation of the BEM model is the convergence of thrust and displacement values as the BEM mesh is refined. Interestingly, $N_{\psi} / N_{e}$ combinations $20 / 80$ and 30/80 have comparable values to 20/160 and 20/320, which could indicate an optimal parameter configuration for simulation performance with acceptable results. 


\section{Case 6.3 Rotor Thrust With Cross-wind}

This Sub-case builds on Sub-case 6.2 with the addition of a relative headwind increasing to $0.5 \mathrm{~m} / \mathrm{s}$ from 0 to 2 seconds. All other time-varying parameters are presented in Figure B.2 in Appendix B.2. A more refined set of $N_{\psi} / N_{e}$ combinations is tested. Due to the headwind, it is expected that the advancing rotor blades see an increase in thrust generation while the retreating blades see a loss in thrust generation which should cause a rolling moment on the airframe. As a result the aircraft will exhibit some translational motion relative to the ship deck.
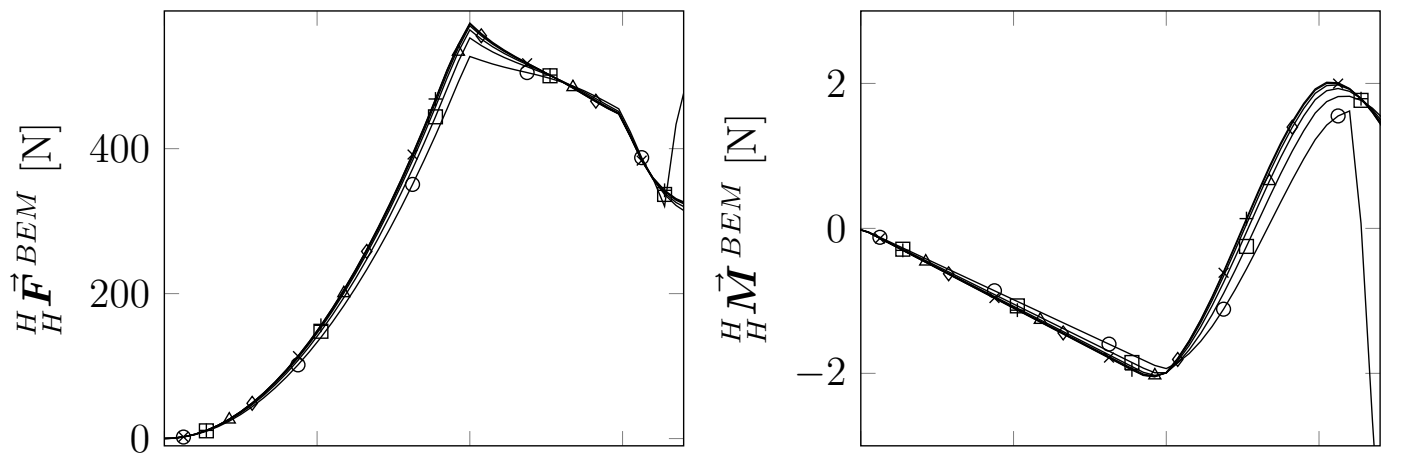

$n_{\text {sec }} / n_{R B E}$

$\multimap 20 / 10$

$\square-20 / 20$

$\triangle 20 / 40$

$\rightarrow 20 / 80$

$\times 20 / 160$

$+20 / 320$
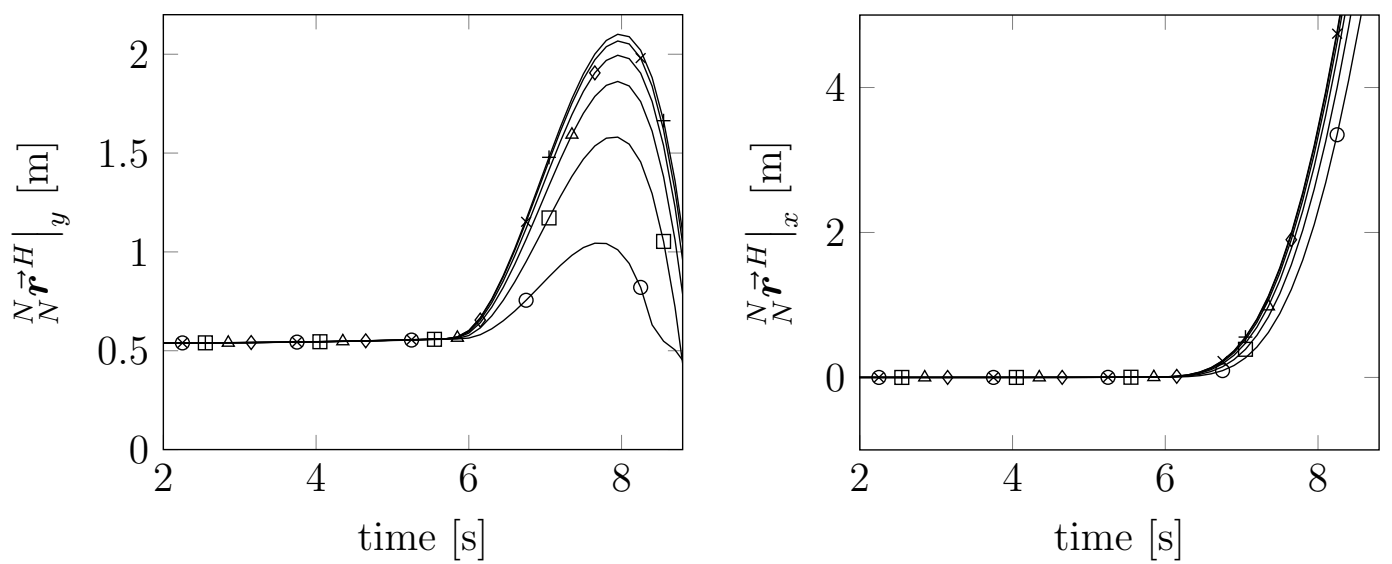

Figure 6.24: Case 6.3 Rotor disc thrust and rolling moment, and airframe CoG position relative to the RSD deck.

The uneven forces on the advancing and retreating blades are inferred from the non-zero rolling moment of the rotor disc acting on the airframe ${ }_{H}^{H} \overrightarrow{\boldsymbol{M}}^{R}$. Even so, the rotor disc generates enough thrust for the aircraft to take-off. Immediately after take-off, the rolling moment causes the aircraft to roll in the clockwise direction. This roll causes the thrust vector to develop a horizontal component relative to the inertial frame which results in the sway of the aircraft. At 8.8 seconds, the aircraft exceeds 
5 metres of horizontal displacement $\left(\left.{ }_{N}^{N} \overrightarrow{\boldsymbol{r}}^{H}\right|_{x}\right)$, which is beyond the edge of the defined ship polygon. This accounts for the large drop in vertical displacement and thrust and moment spikes as the aircraft has fallen off the ship.

Nevertheless, in the planar case, the advancing blades are on the left side of the aircraft, coming out of plane. The higher forces on the advancing side cause negative moment as predicted while on deck from 0 to 6 seconds. Once in the air, the negative rolling moment causes the aircraft to invert to the point where a positve rollingmoment is generated.

The non-zero rolling moment due to uneven rotor disc forces verifies the interaction of the rotor disc with apparent winds, and generates the expected aircraft response to the rotor disc loading. The kinematic development of the BEM model (Eqs. 4.65-4.67) is then implemented correctly.

\section{Case 6.4 Rotor Thrust With Cross-wind and Cyclic Compensation}

Sub-case 6.4 is identical to Sub-case 6.3 with the exception of the cyclic pitch setting function. This Sub-case aims to verify the correct implementation of the cyclical pitch setting for the rotor blades. The cyclical pitch setting causes second harmonic variation of the total blade pitch about one revolution of the rotor disc. The cyclical settings can be used to counteract uneven rotor disc loading. The time varying parameters for this Sub-case are found in Figure B.3 in Appendix B.3.

At 7 seconds, when the rotor has reached peak RPM, there is an increase in collective setting to 0.145 radians, and a cyclic setting of 0.005 radians at maximum azimuth $\pi / 2$. Effectively, this sets the advancing blade minimum pitch to 0.14 radians, and the retreating blade maximum pitch to 0.15 radians. This counteracts the negative roll of the aircraft by generating a large positive rolling moment as shown in Figure 6.25. This causes the aircraft to level out and limit horizontal translation as indicated in the bottom right of Figure 6.25. The aircraft is able to touch back down onto the RSD deck, similarly to Sub-case 6.2. However, since the rotor blades are still spinning and the cyclical setting compensation has subsided, the aircraft begins to exhibit a response similar to Sub-case 6.3 to the point where the aircraft translates until it falls off the ship around 11 seconds.

The response exhibited in this Sub-case verifies the proper implementation of the cyclical pitch function. Prescribing maximum cyclic pitch at the retreating blades allowed for compensation of uneven rotor disc forces due to apparent wind, and 

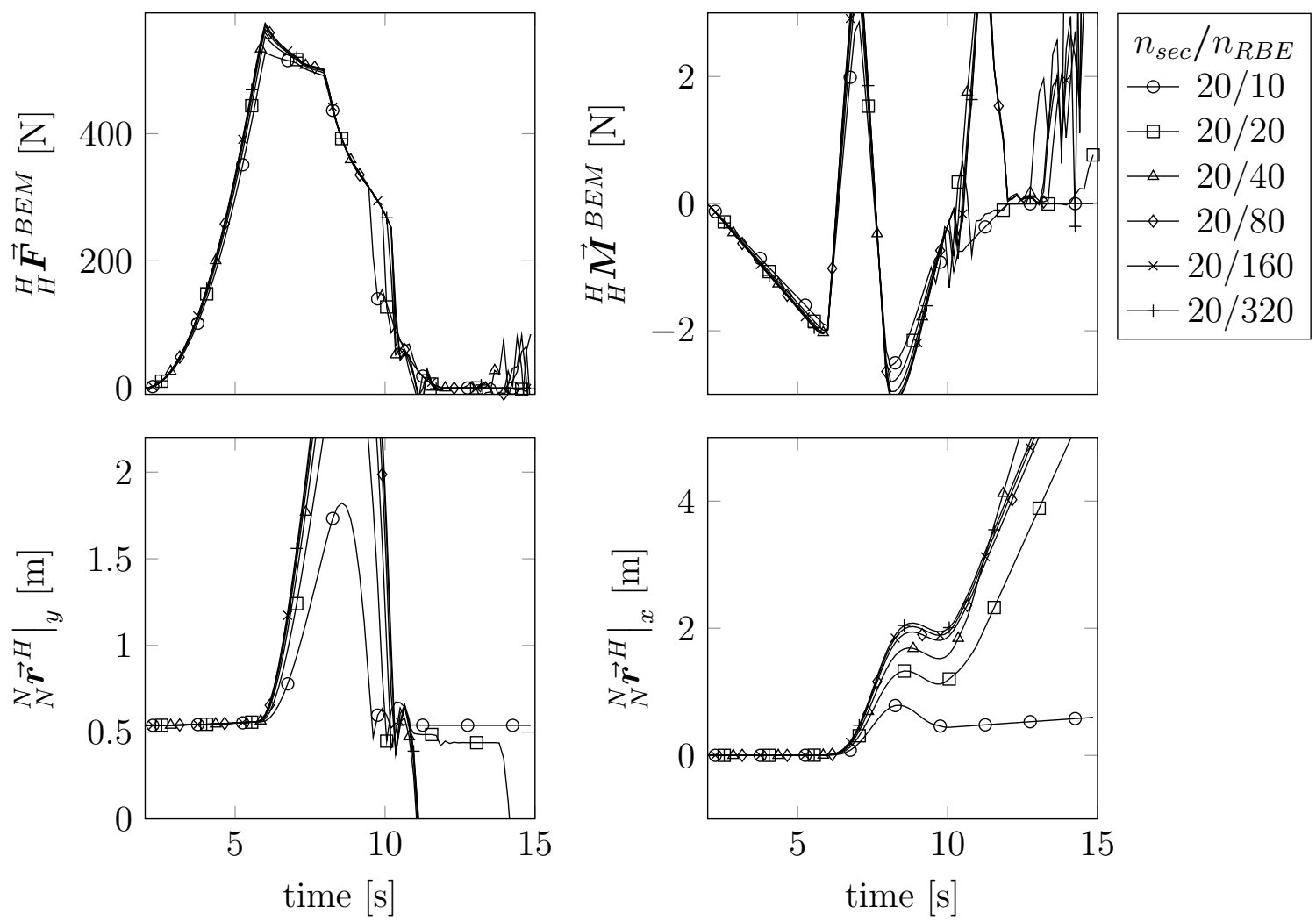

Figure 6.25: Case 6.4 Rotor disc thrust and rolling moment, and airframe CoG position relative to the $\mathrm{RSD}$ deck.

ultimately to corrects the roll of the aircraft for the time period where it is active. 


\section{Case 6.5 Blade Element Model Validation}

The BEM model implemented in SRAMSS was developed in three dimensions. While the scope of this work is mostly limited to verification of the planar version of SRAMSS, the BEM model is independently validated against published experimental data.

During the eighties, comprehensive experimental testing of the UH-60A helicopter rotor blades had been undertaken. Enough published data exists to create a blade model for SRAMSS. The BEM model implemented in SRAMSS is evaluated against CFD model data presented in Garcia and Barakos [7], and the experimental data to which it is evaluated from Balch et al [8]. The blade profile used in these experiments is presented in Figures C.1 and C.2, and the equivalent rotor blade profile used in SRAMSS is presented in Figure C.3 with additional parameters presented in Table C.1. These figures and tables are found in Appendix C. The SRAMSS blade profile was modelled from the profile presented in Garcia and Barkados since it is more detailed. $\frac{\mathrm{d} C_{L}}{\mathrm{~d} \alpha}\left(M=0, R e=10^{6}\right)$ and $\frac{\mathrm{d} C_{D}}{\mathrm{~d} \alpha}\left(M=0, R e=10^{6}\right)$ are obtained from XFoil-generated data published at Airfoiltool.com [59] and corroborated by Totah [60].

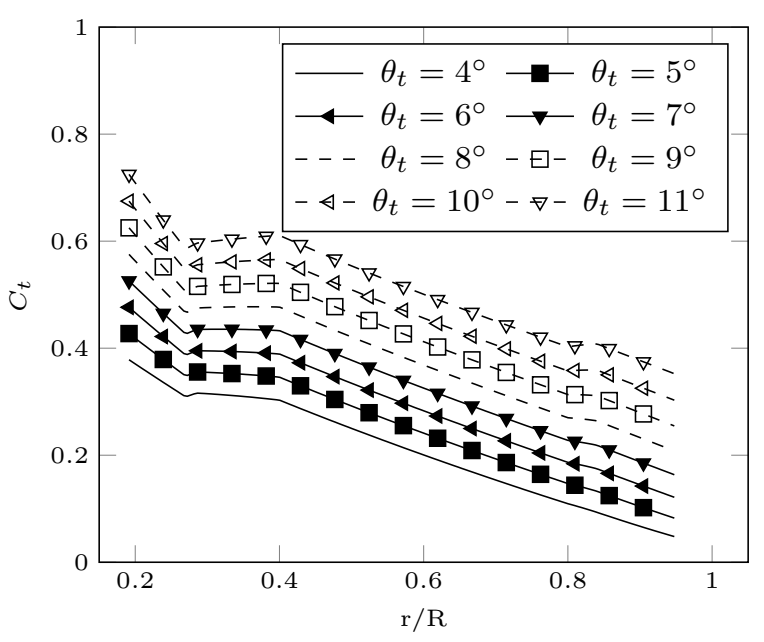

(a) SRAMSS S-76 BEM model

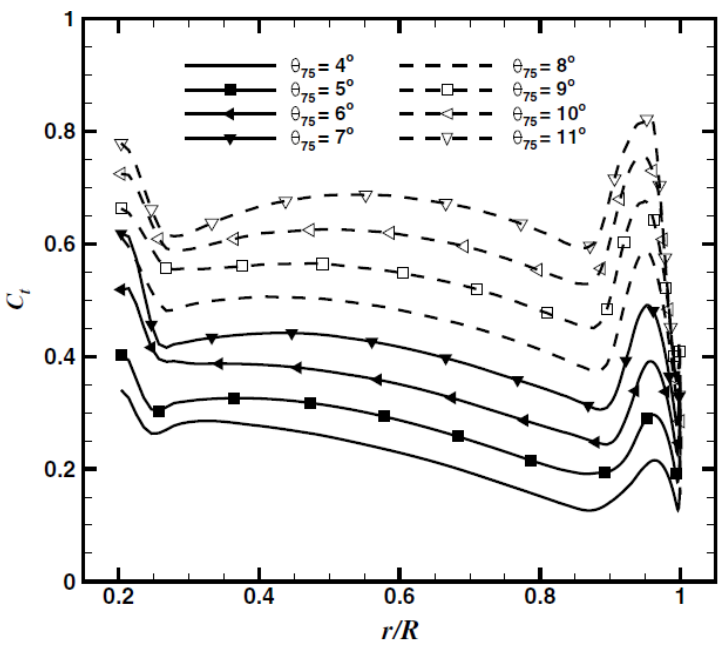

(b) Garcia and Barakos [7]

Figure 6.26: Case 6.5 Comparison of blade section thrust coefficient between SRAMSS BEM model and CFD analysis from Garcia and Barakos.

Given that the blade is divided into elements for the BEM model, the sectional thrust coefficient $C_{t}$, a non-dimensional measure of the generated thrust, is calculated 
as $C_{t}=\mathrm{d} T /\left(\rho_{\text {air }}(\Omega r)^{2} c \mathrm{~d} r\right)$. As shown in Figure 6.26, the BEM model exhibits similar blade section thrust coefficient variation to the results from Garcia and Barakos for a 1/4.71-scale S-76 rotor in hover, with blade-tip Mach number $M_{t i p}=0.65$. However, the SRAMSS BEM model overestimates $C_{t}$ near the root of the blade, and underestimates $C_{t}$ closer to the blade tip, where complex blade-tip geometries are present.

Discrepancies between the two models arise from their core differences. The SRAMSS BEM model, still in its developmental phase, is a basic lift and momentumbased elemental approach which has minimal corrections for aerodynamic phenomena. Whereas the work done by Garcia and Barakos is based in high-fidelity computational fluid dynamics (CFD). The discrepancies in the estimation of $C_{t}$ are most likely due to the simplistic calculation of the coefficient of drag for the BEM model. The linear drag coefficient model does not capture the parabolic nature of drag under different flight speeds where either parasitic drag or lift-induced drag dominate. Additionally, no effort is made to consider the drag-divergence Mach number where there is a rapid increase in drag coefficient, such as Figure 2.4 of Keys [61]. The limited consideration of drag overestimates the value of thrust in calculation per Equation 4.88, which affects $C_{t}$ accordingly.

With respect to the CFD analysis, blade wake, wing tip vortices, and wing tip shape are considerations not modelled by the SRAMSS BEM model. These ultimately affect the sectional thrust coefficient. Specifically, the wing tip modelling, and wing tip vortices are reasoned to influence the $C_{t}$ values at the wing tips according to Garcia and Barakos.

Another estimation of performance for the aircraft in hover, is the figure of merit (FoM). The BEM model is matched closest to the rectangular, flat-cap tip blade model from Garcia and Barakos. For tip Mach-numbers of $M_{t i p}=0.65$ and $M_{t i p}=$ 0.60, the FoM versus blade loading coefficient $C_{T} / \sigma$ for the BEM model and CFD analyses are given in Figures 6.27 and 6.28. The rotor thrust coefficient is $C_{T}=$ $T /\left(\rho_{\text {air }}(\Omega R)^{2} \pi R^{2}\right)$, where $\sigma$ is the rotor solidity ratio. For the BEM model, the momentum-based estimation of the FoM is

$$
\mathrm{FoM}=\frac{C_{T}^{3 / 2}}{\sqrt{2}\left(\sigma\left(C_{D 0} / 8\right)+k_{i}\left(C_{T}^{3 / 2} / \sqrt{2}\right)\right)}
$$

where $k_{i}=1.1$ is the induced power factor, and $C_{D 0}=0.01$ is the overall profile drag coefficient [7]. 


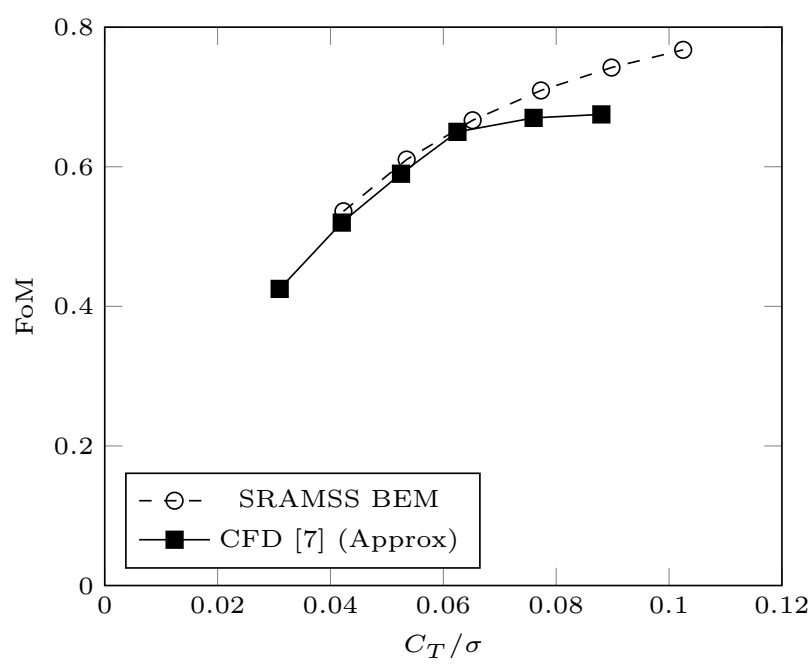

(a) SRAMSS S-76 BEM model

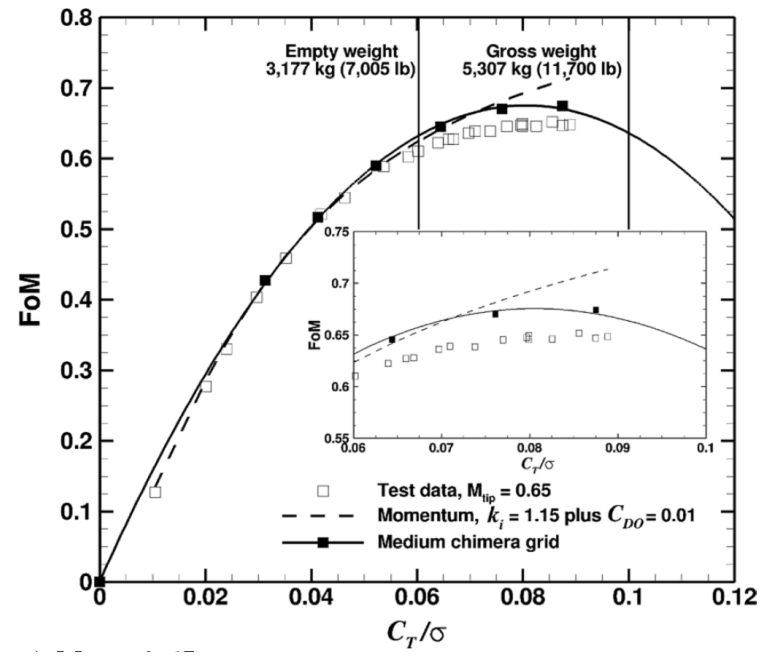

(b) Garcia and Barakos [7]

Figure 6.27: Case 6.5 Comparison of FoM for SRAMSS BEM and CFD model. $M_{\text {tip }}=$ $0.65, \theta_{t}=\left\{4^{\circ}, 5^{\circ}, 6^{\circ}, 7^{\circ}, 8^{\circ}, 9^{\circ}\right\}$.

Similar to the sectional thrust coefficients, the rotor thrust coefficient is also overestimated, which leads to an overestimation in the momentum-based FoM number. This overestimation causes a shift in the FoM values predicted by the BEM model. While there is some variation with respect to the values obtained through CFD analysis, The BEM model values align with the momentum trends from Figures 6.27b and $6.28 \mathrm{~b}$ which supports the proper implementation of the BEM model in SRAMSS.

The simulations performed for Sub-cases 6.2 through 6.4 verify that the BEM model for rotor disc forces has been implemented correctly. The rotor generates appropriately-oriented thrust vectors and roll moments to the airframe in various wind conditions.

Initial validation of the BEM model in Sub-case 6.5 reveals that the trends in performance measures are similar to CFD and experimental values, yet highlights the need for more accurate modelling of aerodynamic phenomena at the rotor disc. The accuracy required of the BEM model for dynamic interface analysis, can only be determined by the comparison of full three-dimensional simulations against experimental data. 


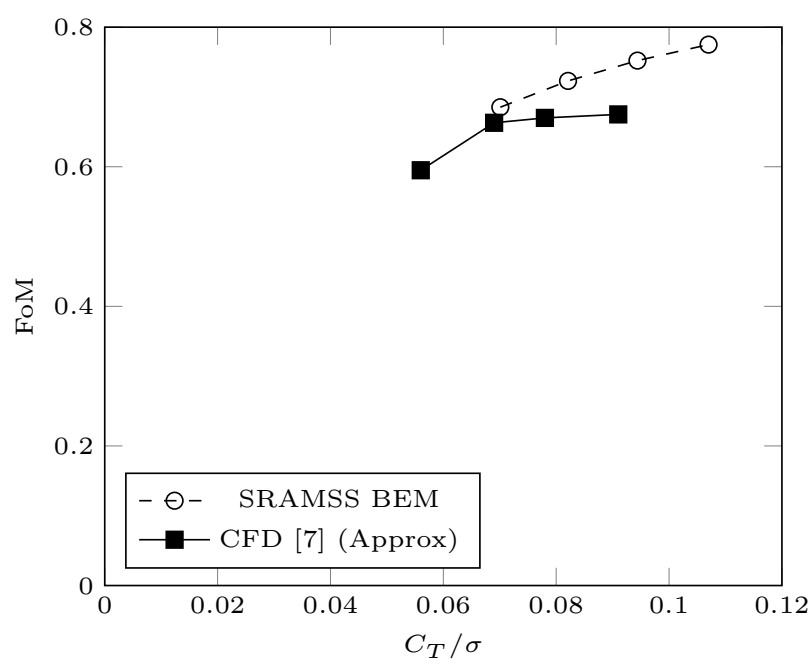

(a) SRAMSS S-76 BEM model

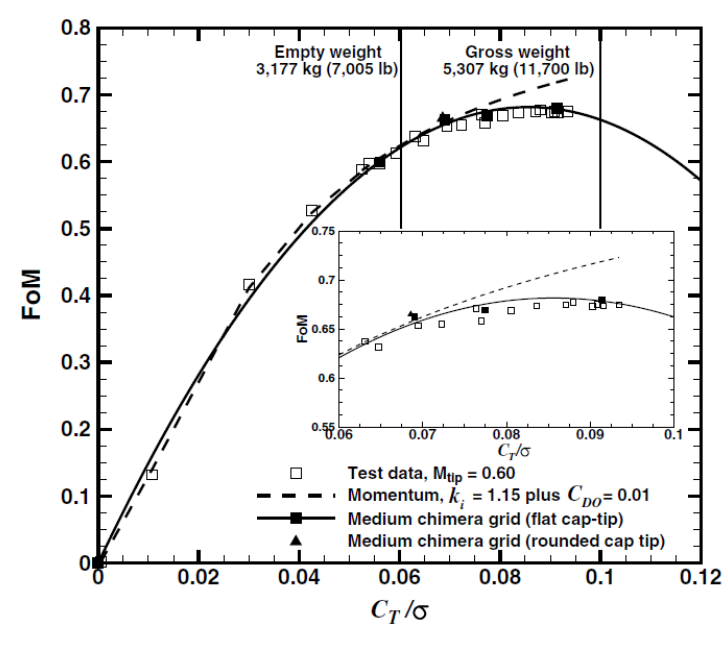

(b) Garcia and Barakos [7]

Figure 6.28: Case 6.5 Comparison of FoM for SRAMSS BEM and CFD model. $M_{\text {tip }}=$ $0.60, \theta_{t}=\left\{6.5^{\circ}, 7.5^{\circ}, 8.5^{\circ}, 9.5^{\circ}\right\}$.

\subsubsection{Case 7: Securing Concept Verification}

Having shown that the contact dynamics model generates an appropriately-oriented dynamic response to collision through the simulations performed in Case 5 in Section 6.3.5, the wedge clamps as a skid-equipped aircraft securing device can be qualified.

Case 7 is split into static and dynamic Sub-cases. First, the static Sub-case 7.1 verifies that the aircraft reacts appropriately to the securing device on a stationary ship. Second, the dynamic Sub-case 7.2 verifies the securing device's ability to secure and manoeuvre the aircraft under various prescribed ship and RSD deck motions.

\section{Case 7.1 Static Aircraft Securing}

For Sub-case 7.1 the generic aircraft is secured to the RSD deck under three different initial conditions. In the first simulations, the aircraft undergoes a level drop towards the RSD deck, while being on-centre with respect to the RSD, where it is then secured by the device. Next, the aircraft is similarly dropped, but off-centre from the RSD. Lastly, the aircraft is dropped onto a ship with a fixed roll angle. In all simulations, the securing device is 'activated' at 1 second of simulation time, and requires 0.5 seconds to spread the wedge clamps. These configurations are illustrated in Figure 6.29.

It is convenient to quantify the device's ability to secure the aircraft by evaluating 


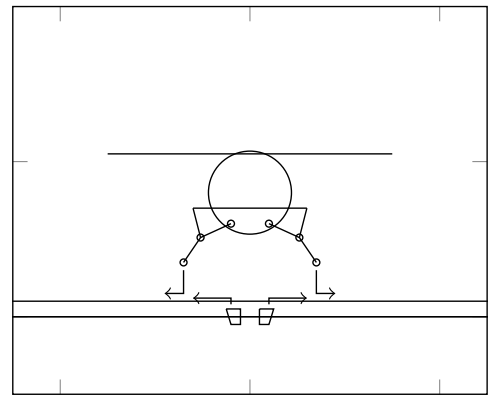

On-centre

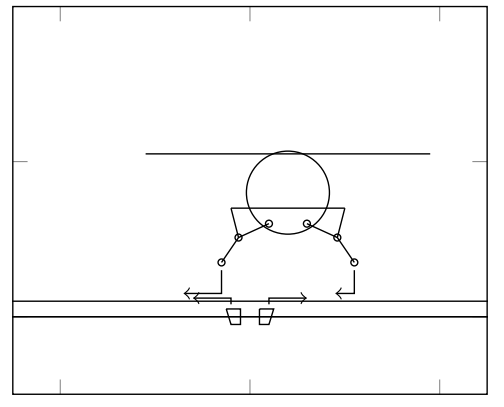

Off-centre

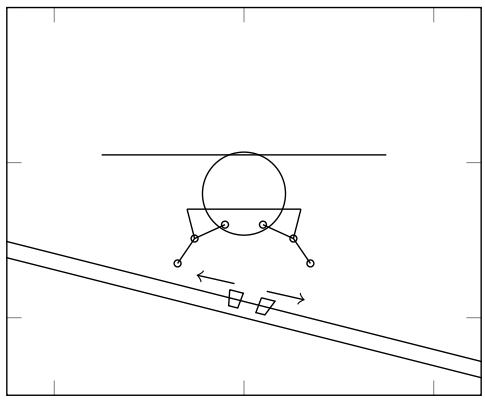

Rolled Ship

Figure 6.29: Case 7.1 Static aircraft securing simulation configurations.

the magnitude of distances of specific parts of the aircraft with respect to parts of the securing device. Three distances are used to evaluate the performance of the securing device. First, the position of the airframe relative to the RSD frame $\left\|{ }_{N}^{D} \overrightarrow{\boldsymbol{r}}^{H}\right\|$ shows the ability of the RSD deck to manoeuvre a secured aircraft. Second, the distances between the contact nodes and their respective clamping wedges $\left\|_{N}^{C L} \overrightarrow{\boldsymbol{r}}^{n_{1}}\right\|$ and $\left\|_{N}^{C R} \overrightarrow{\boldsymbol{r}}^{n_{6}}\right\|$ show the wedge clamp's ability to retain the skid-tubes. These distances quantify the aircraft's position relative to the securing device, and as such, indicates the ability of the device to retain the aircraft independent of any prescribed ship or RSD deck motion.

These distances are obtained from the kinematic developments of Chapter 3 as follows:

$$
\begin{aligned}
\left\|_{N}^{D} \overrightarrow{\boldsymbol{r}}^{H}\right\| & =\left\|{ }_{N}^{N} \overrightarrow{\boldsymbol{r}}^{H}-{ }_{N}^{N} \overrightarrow{\boldsymbol{r}}^{D}\right\| \\
\left\|_{N}^{C L} \overrightarrow{\boldsymbol{r}}^{n_{1}}\right\| & =\left\|_{N}^{N} \overrightarrow{\boldsymbol{r}}^{n_{1}}-{ }_{N}^{N} \overrightarrow{\boldsymbol{r}}^{C L}\right\| \\
\left\|_{N}^{C R} \overrightarrow{\boldsymbol{r}}^{n_{6}}\right\| & =\left\|_{N}^{N} \overrightarrow{\boldsymbol{r}}^{n_{6}}-{ }_{N}^{N} \overrightarrow{\boldsymbol{r}}^{C R}\right\|
\end{aligned}
$$

The results of the three simulations are presented in Figures 6.30 through 6.32 .

In the first two simulations, the three distances settle to the same values of $\left\|{ }_{N}^{D} \overrightarrow{\boldsymbol{r}}^{H}\right\|=0.4247$ metres, $\left\|{ }_{N}^{C L} \overrightarrow{\boldsymbol{r}}^{n_{1}}\right\|=\left\|_{N}^{C R} \overrightarrow{\boldsymbol{r}}^{n_{6}}\right\|=0.0757$ metres. For the last case, the values settle to $\left\|{ }_{N}^{D} \overrightarrow{\boldsymbol{r}}^{H}\right\|=0.4247$ metres, $\left\|{ }_{N}^{C L} \overrightarrow{\boldsymbol{r}}^{n_{1}}\right\|=0.0756$ metres, and $\left\|{ }_{N}^{C R} \overrightarrow{\boldsymbol{r}}^{n_{6}}\right\|=0.0757$ metres respectively. The difference in the last simulation is attributed to the weight of the aircraft being held at the interface of $n_{1}$ and the left wedge clamp, whereas there is only friction keeping $n_{6}$ from sliding down the inclined RSD deck.

Nevertheless, there is one significant commonality between each simulation. Be- 


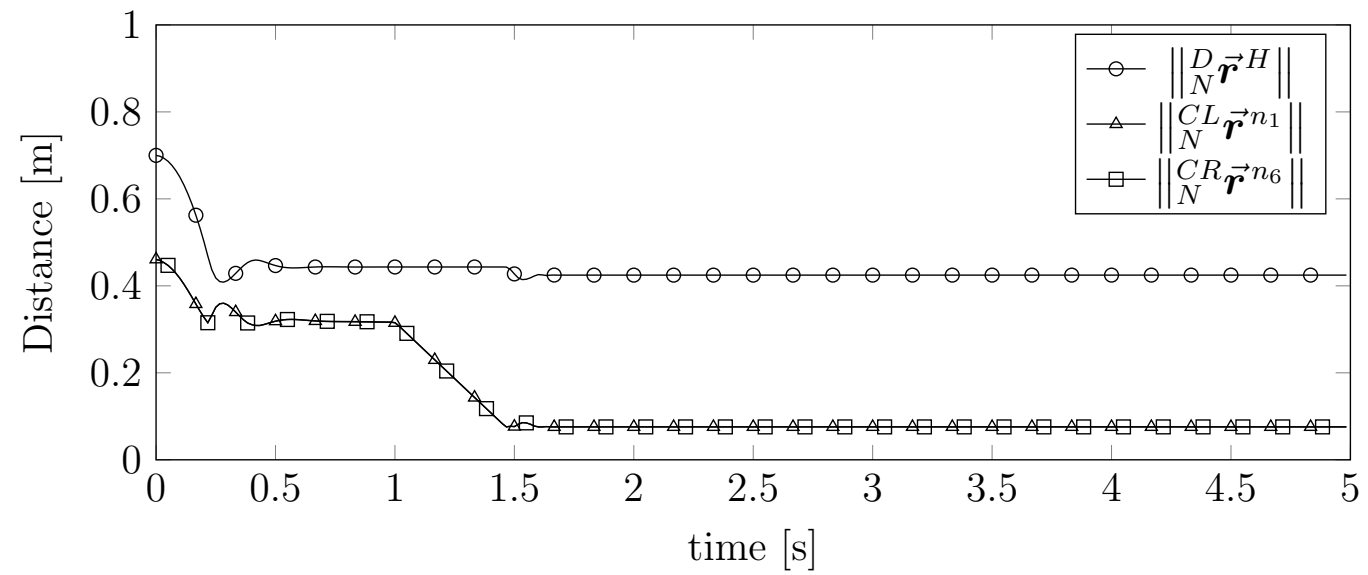

Figure 6.30: Case 7.1 Static aircraft securing in the 'On-centre' scenario.

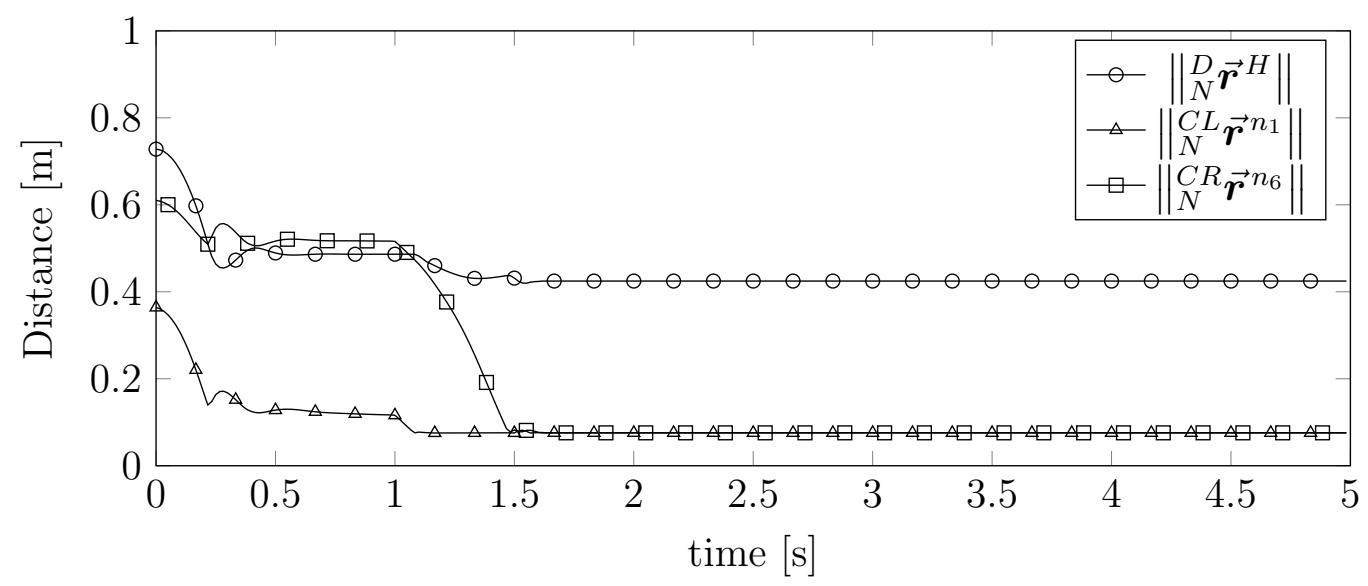

Figure 6.31: Case 7.1 Static aircraft securing in the 'Off-centre' scenario.

tween 1.0 and 1.5 seconds the three distances settle to their final values. This verifies that the aircraft is secured for the static scenarios. By extension, the securing device concept is initially verified. 


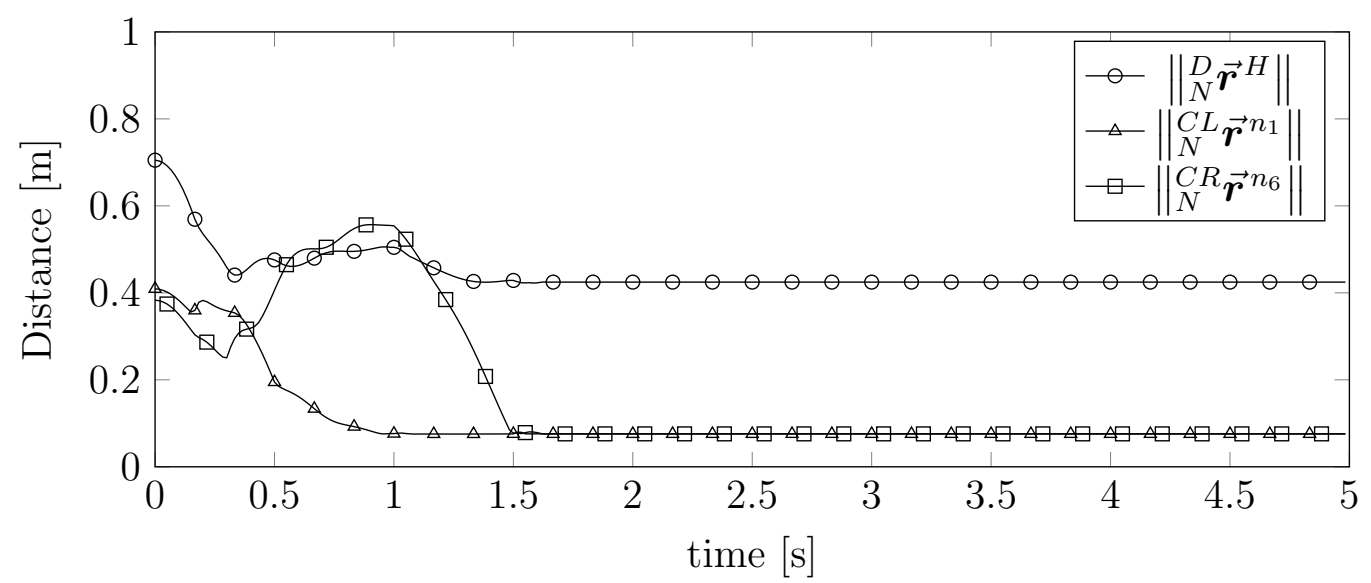

Figure 6.32: Case 7.1 Static aircraft securing in the 'Rolled Ship' scenario.

\section{Case 7.2 Dynamic Aircraft securing}

Under normal operations, the RSD is used to secure an aircraft to a moving ship in extremely dynamic conditions. A similar analysis to that of Sub-case 7.1 is performed for dynamic ship and RSD deck conditions using the same metrics to evaluate aircraft securing. For Sub-case 7.2 there are two simulations. In the first simulation, the aircraft is dropped onto a rolling ship, then is secured at 3 seconds. In the second simulation, the aircraft is secured at 1 second, then manoeuvred across the ship deck by means of the RSD deck. A more complex dynamic securing scenario is simulated for comprehensive testing in Case 8. The two simulation configurations are shown in Figure 6.33.

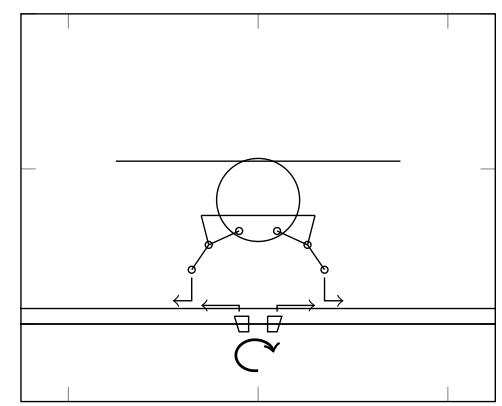

Rolling Ship

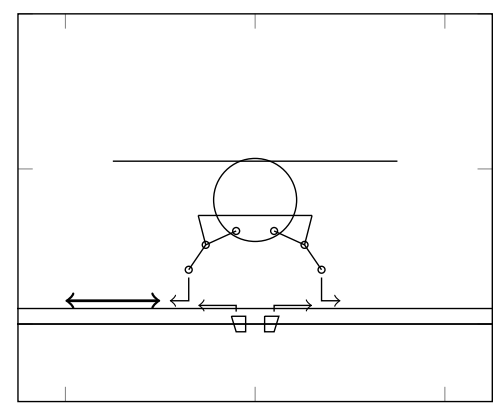

RSD Manoeuvre

Figure 6.33: Case 7.2 Dynamic aircraft securing simulation configurations.

The results of the two dynamic simulations are presented in Figures 6.34 through 6.37. As with the static tests, once the aircraft is secured, the distances are maintained at 
the expected values which verifies that the securing device is able to initially secure and keep secured the aircraft under dynamic conditions.

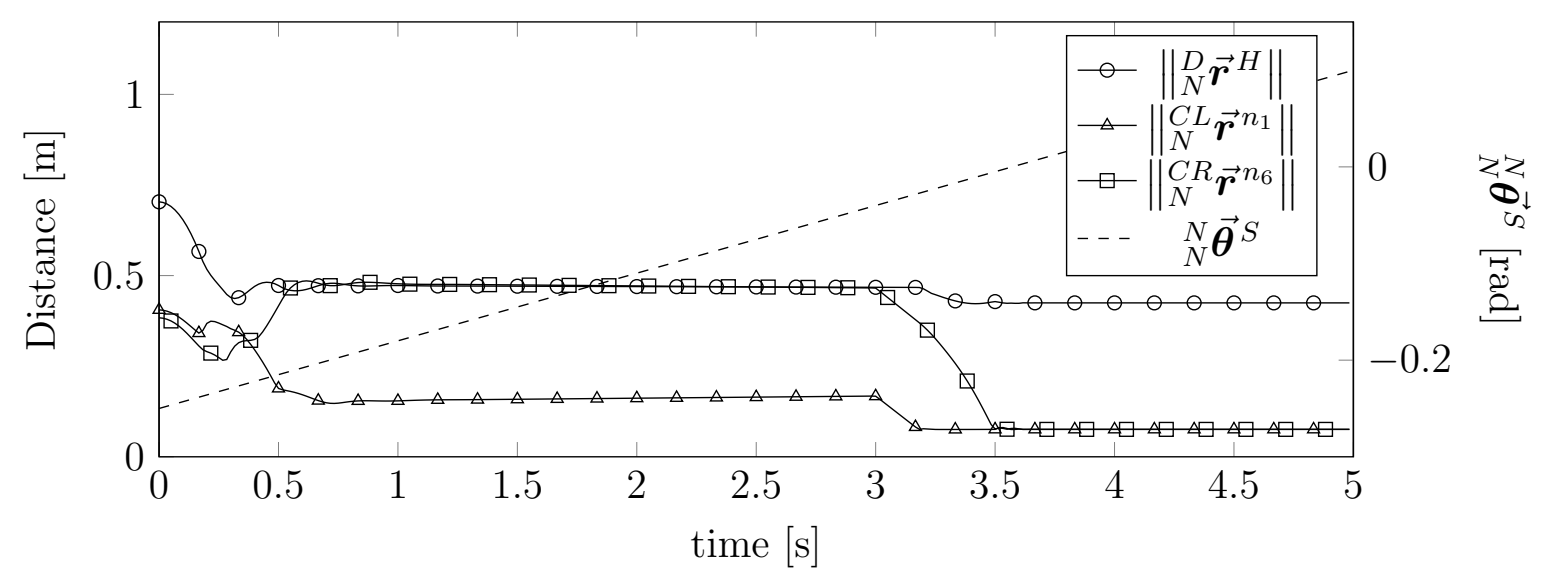

Figure 6.34: Case 7.2 Dynamic aircraft securing in the 'Rolling Ship' scenario.

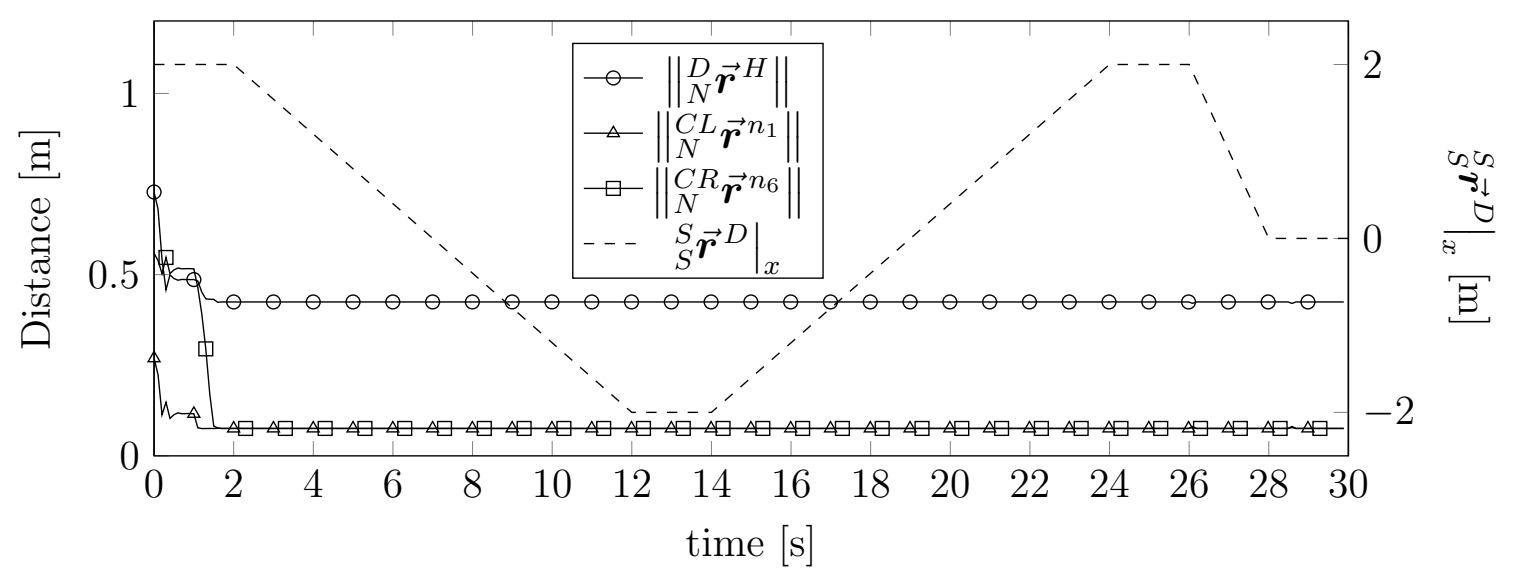

Figure 6.35: Case 7.2 Dynamic aircraft securing in the 'RSD Manoeuvre' scenario.

Figures 6.36 and 6.37 show the magnitude of the contact forces acting at the contact nodes in both tests. The data indicates that the trailing wedge clamp on deceleration of the RSD deck, and the leading wedge clamp on acceleration generate larger contact forces.

In any case, under static and dynamic conditions, Sub-cases 7.1 and 7.2 verify the implementation of the securing device conceptual design presented in this work. It further verifies the contact dynamics model, as the RSD deck, and wedge clamps are polygons defined in the SRAMSS environment. Moreover, it suggests that the concept is effective and warrants further investigation and design refinement. 


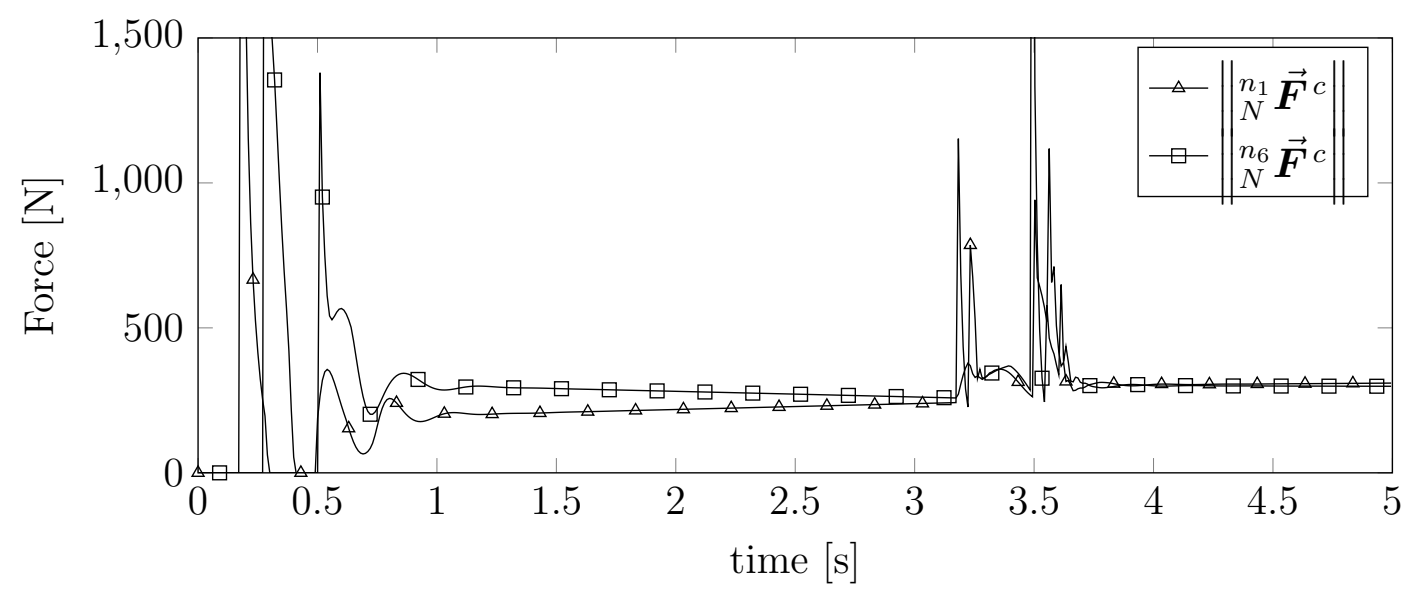

Figure 6.36: Case 7.2 Contact force magnitudes in the 'Rolling Ship' scenario.

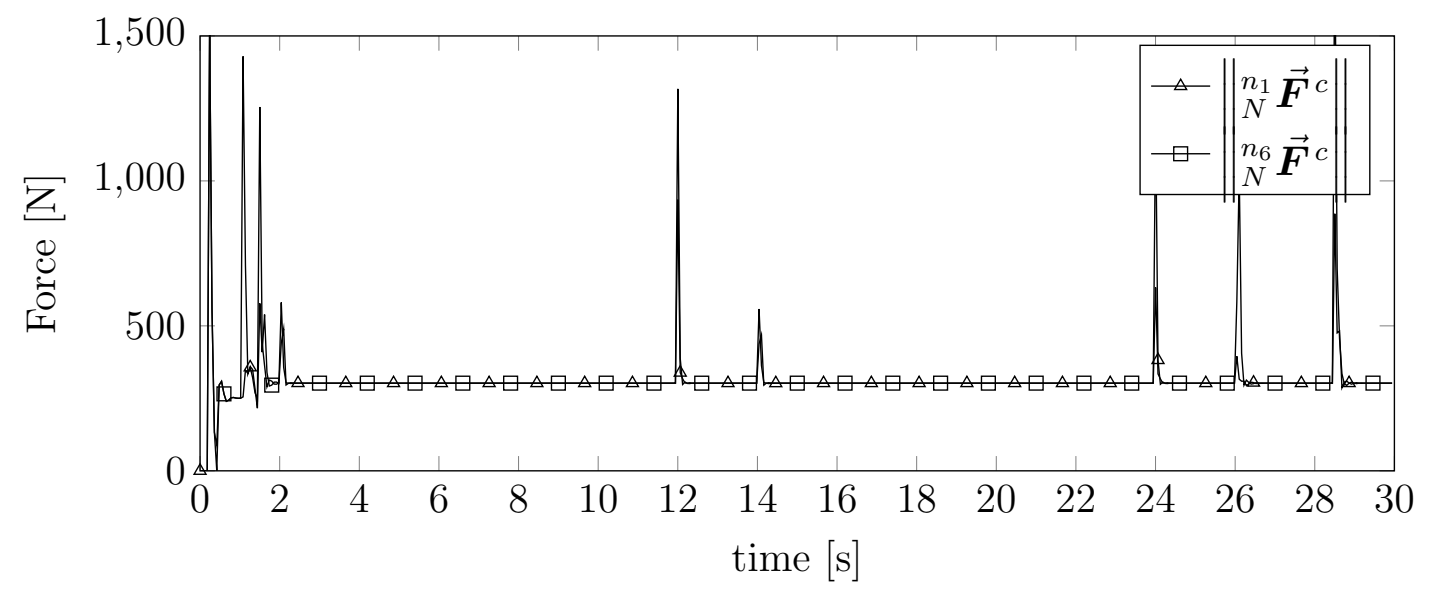

Figure 6.37: Case 7.2 Contact force magnitudes in the 'RSD Manoeuvre' scenario.

\subsubsection{Case 8: Comprehensive Phenomena Verification}

At this point, all models implemented in SRAMSS have been isolated to the extent possible for independent evaluation. All models have been verified to be properly implemented in the planar version of SRAMSS. Yet in comprehensive simulation conditions, simultaneous functioning of all models is required.

Case 8 incorporates the complete recovery flight, securing, manoeuvring, and launch phases of shipboard helicopter operations. Aircraft flight and securing system control is outside of this work, therefore prescribed aerodynamic parameters and polyogn motions were iterated to obtain the desired visual behaviour. First, the aircraft enters recovery flight where it station-keeps over a ship deck moving all three degrees of freedom, while correcting its flight attitude against head and cross-winds. 
When ship-motion velocity slows, the aircraft lands on the ship where it is then secured to the RSD deck via the wedge clamp securing device. Once secured, the aircraft is powered-down. Then, the RSD performs a translational manoeuvre of the embarked aircraft to simulate secured aircraft manoeuvring under large ship motions. Near the end of the manoeuvre, large cross-winds act on the aircraft. Finally, the aircraft begins pre-flight power-up, and is eventually released from the securing device to take-off in headwinds.

Simulation-time-varying parameters are presented in Figures D.1 and D.2 in Appendix D. The scenario is run for all combinations of $N_{n}=\{6,10,14,18\}$ by $N_{\psi}=\{20\}$ by $N_{e}=\{40,80,160\}$ over a 47 -second simulated time period.

Figure 6.38 showcases eventful timestamps in the Case 8 simulation. At 0 seconds, headwinds cause the aircraft to roll and sway to the right. The roll is overcompensated by cyclical pitch settings at 1 second which causes rolling in the opposite direction. Sway in the opposing direction is prevented by correction to the cyclical pitch settings and large cross-winds. Around 6 seconds, the aircraft lands on the now-level ship deck. The securing device and RSD deck capture the landed aircraft at 7 seconds. Between then and 10 seconds, the aircraft powers down. The RSD performs translational manoeuvres of the embarked aircraft until 33 seconds while the ship heaves, sways, and rolls. Near the end of the manoeuvres, a large gust of wind crosses the ship. Power-up of the aircraft begins at 39 seconds. Around 42 seconds, the aircraft reaches full power, with collective pitch set to its maximum value, then is immediately released. Beyond 43 seconds, the aircraft performs manoeuvres to exit the immediate area of the ship.

The transient position of the aircraft's airframe is shown in Figure 6.39. There are two major observations which can be made. First, the refinement of the BEM mesh has greater influence on aircraft response than DFE mesh refinement since the plot lines appear to be superimposed for $N_{e}$. This is expected as external-damperequipped DFE models showed little difference in response for the 10-, 14-, and 18-node meshes as illustrated in Case 4.2's Figure 6.13, and convergence of results for different BEM meshes is slower as Case 6.4's Figure 6.25 shows. Second, the discrepancy in response for combinations where $N_{e}=40$ causes the aircraft to miss the securing device (for equal prescribed motions and time-varying parameters). Interestingly, when the securing device activates, the left contact node is wedged against the right wedge clamp. Then, when the collective setting is increased to 0.14 radians after 
40 seconds, enough lift is generated to take off. However, since the left node is wedged, the generated thrust causes the aircraft to pivot about the right wedge clamps and roll over into the RSD deck as shown by the drop in $y$-position and spike in $x$-position around the 42-second mark in Figure 6.39.

Referencing to the 'complex dynamic securing scenario' mentioned in Section 6.3.7, the same analysis can be done to verify the RSD's performance for Case 8's fullydynamic scenario. Figure 6.40 shows the measured distances, while Figure 6.41 shows the magnitude of the corresponding contact forces. Figure 6.40 corroborates the observation that the $N_{e}=40$ combinations fail to secure to the RSD. For the combinations where $N_{e} \neq 40$, Figure 6.40 settles to values similar to those in Figures 6.29 through 6.35 of Case 7 under secured conditions.

With respect to the contact forces, Figure 6.41 follows the trend that the results are nearly identical for combinations where $N_{e} \neq 40$. For the tests where the aircraft has been secured to the ship between 7 and 42 seconds, there are fluctuations in the contact forces due to the shift in aircraft weight as the ship moves. For the unsecured aircraft, the fluctuations are more apparent since friction is the dominating force keeping the aircraft on deck. Correspondingly, in Figure 6.40, $\left\|{ }_{N}^{C L} \overrightarrow{\boldsymbol{r}}^{n_{1}}\right\|$ and $\left\|{ }_{N}^{C R} \overrightarrow{\boldsymbol{r}}^{n_{6}}\right\|$ are near-constant for the secured condition, while $\left\|{ }_{N}^{C L} \overrightarrow{\boldsymbol{r}}^{n_{1}}\right\|$ and $\left\|_{N}^{C R} \overrightarrow{\boldsymbol{r}}^{n_{6}}\right\|$ show slight deviations for the unsecured condition. This suggests that the aircraft is beginning to slide in the unsecured condition.

The thrust and rolling moment generated by the rotor disc are the major forces governing the response of the aircraft while not secured. Figure 6.42 shows their magnitudes for the duration of the simulations.

The roll corrections made by the aircraft in the recovery flight phase correspond to the reversals in the values for $\left.{ }_{H}^{H} \overrightarrow{\boldsymbol{M}}^{B E M}\right|_{y, 2 D}$. Between 31 and 33 seconds a large gust of wind crosses the ship. While the rotor blades are not spinning, the apparent wind across the rotor disc does generate some value for thrust and rolling-moments which affect the aircraft. In Figure 6.42, when the aircraft is released from the RSD at 42 seconds, there is a drop in thrust from roughly $500 \mathrm{~N}$ to $200 \mathrm{~N}$. For the unsecured aircraft, the thrust drops down to $200 \mathrm{~N}$ as it rolls over, then jumps to $500 \mathrm{~N}$ once inverted.

Case 8 comprehensively tests all models implemented in SRAMSS concurrently. The results presented for Case 8 match those of independently evaluated models in preceding cases, which verifies that the models are able to function properly in fully- 
dynamic environments. Moreover, Case 8 has verified that the contact dynamics model functions properly for multiple polygons in the same simulation, and for contact states where the contact node is in contact with more than one polygon at a time.

At this point, all models contained in the Planar version of SRAMSS are verified. Consequently, it lays the architectural foundation and system formulation for a future fully-spatial version of SRAMSS. 

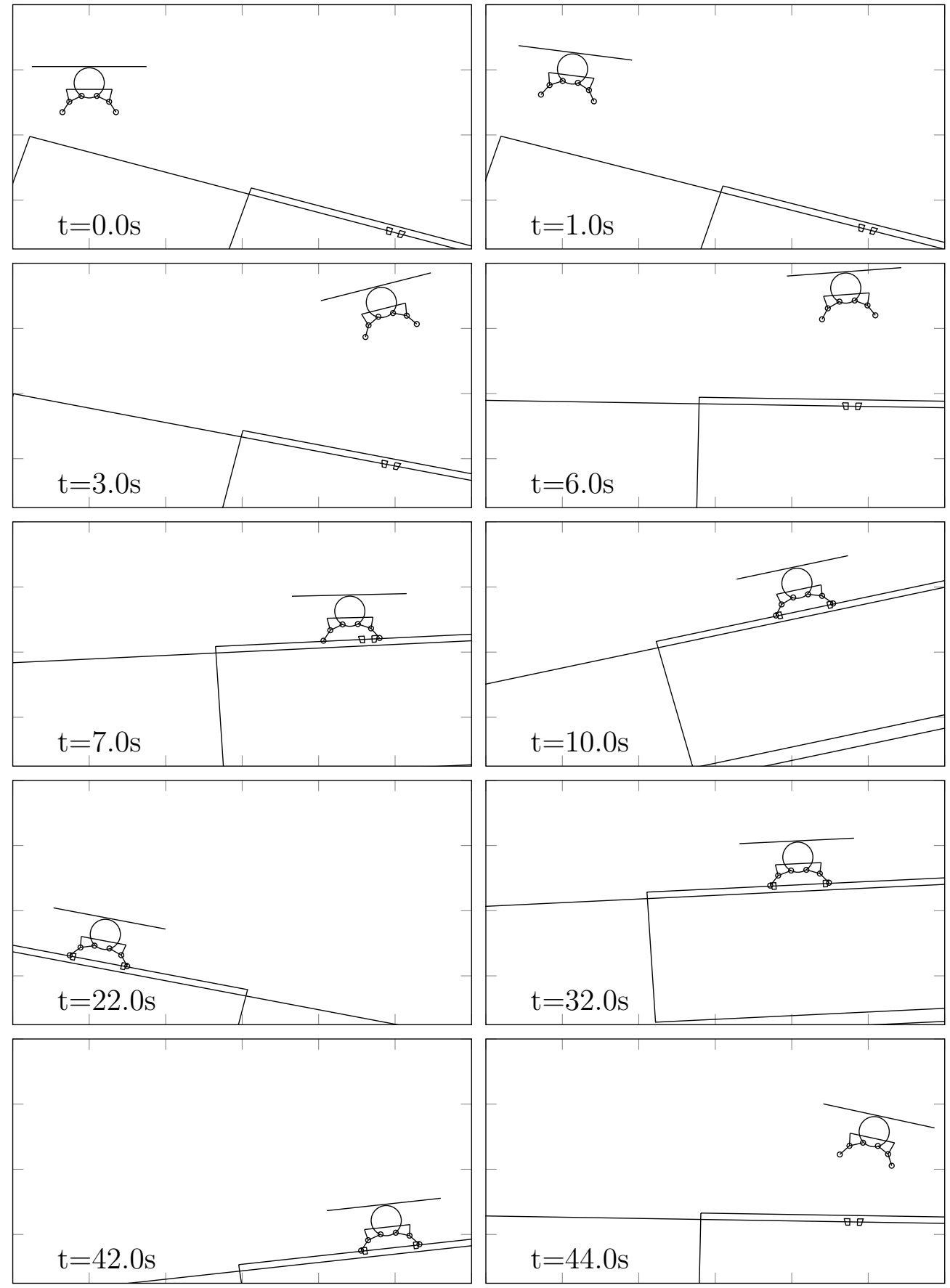

Figure 6.38: Case 8 Comprehensive simulation time-stamped event animations. 


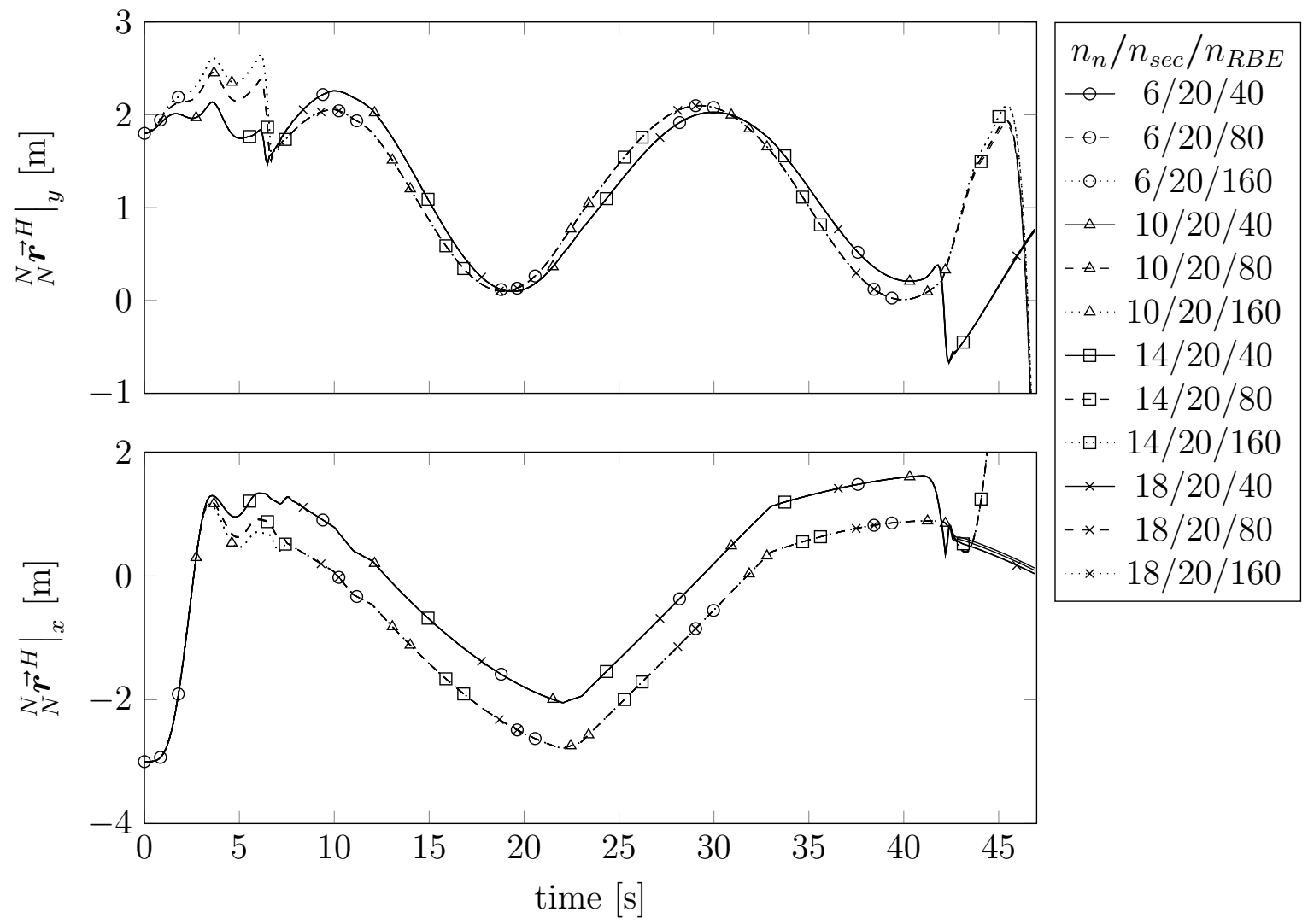

Figure 6.39: Case 8 Airframe CoG position versus time for all combinations of $n_{n} / N_{\psi} / N_{e}$ 


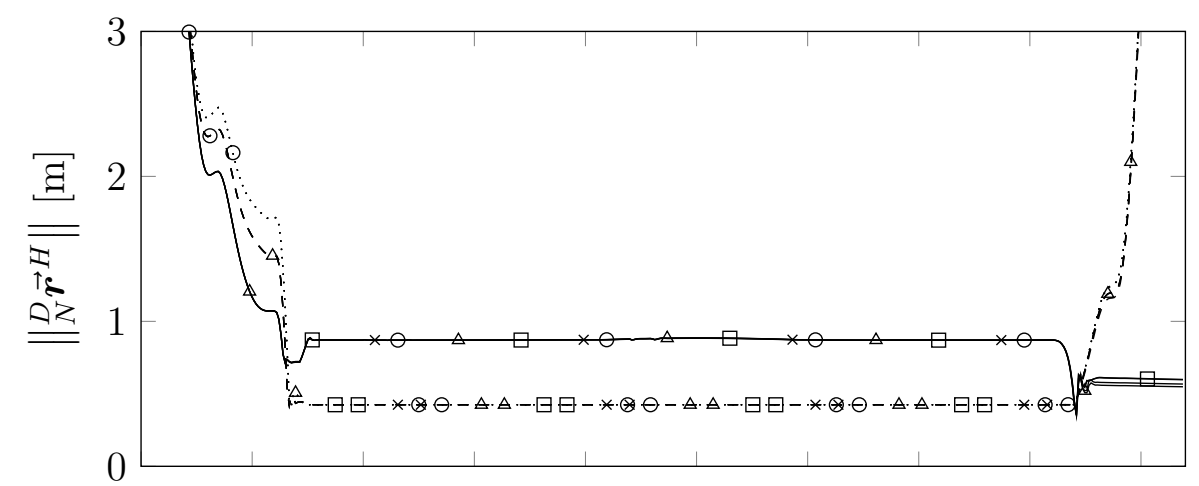

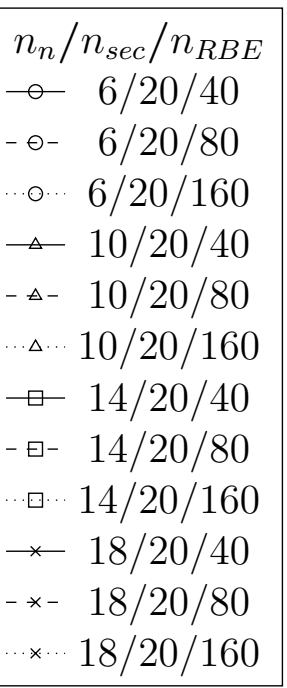
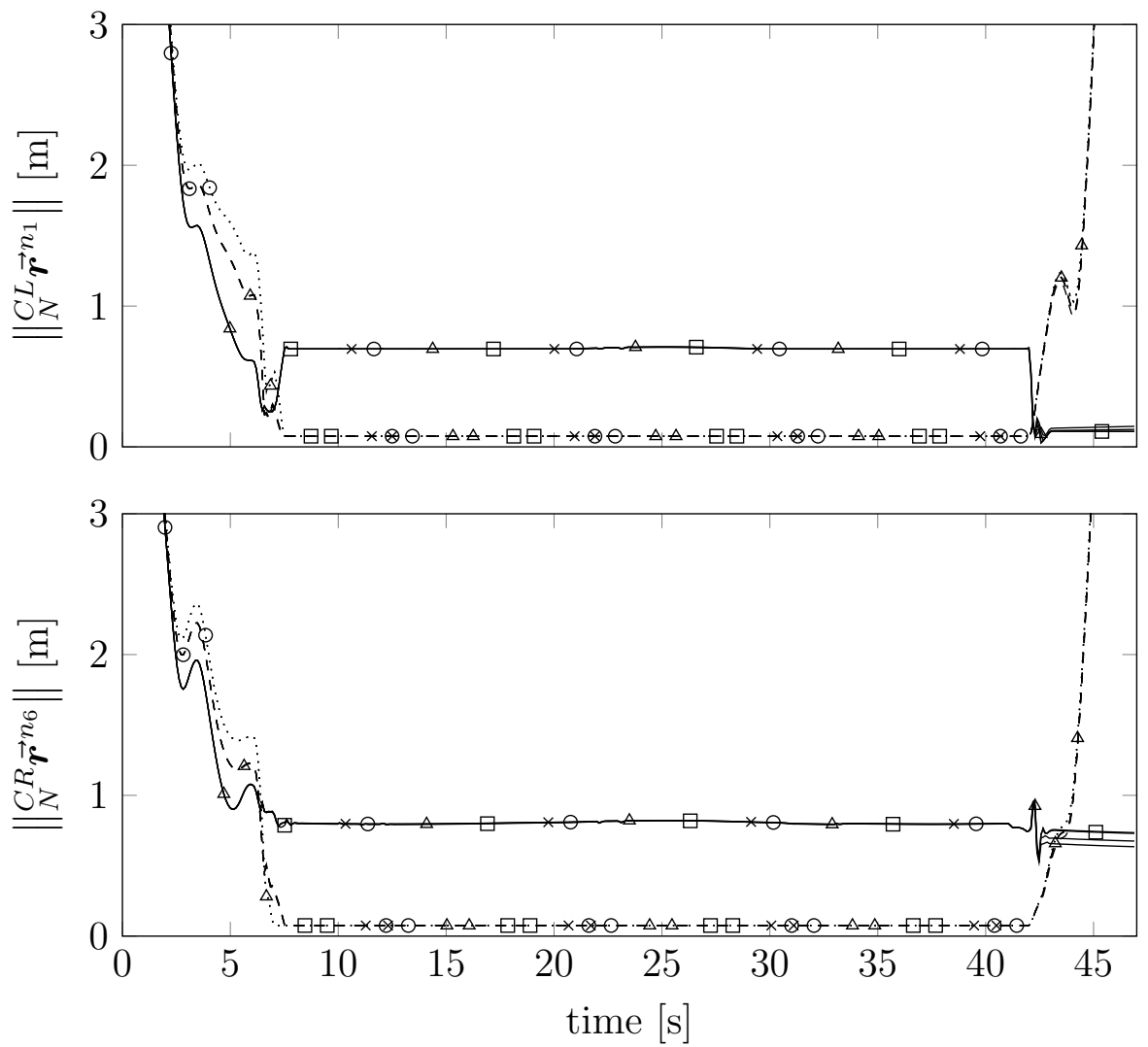

Figure 6.40: Case 8 Aircraft distances relative to RSD points. 


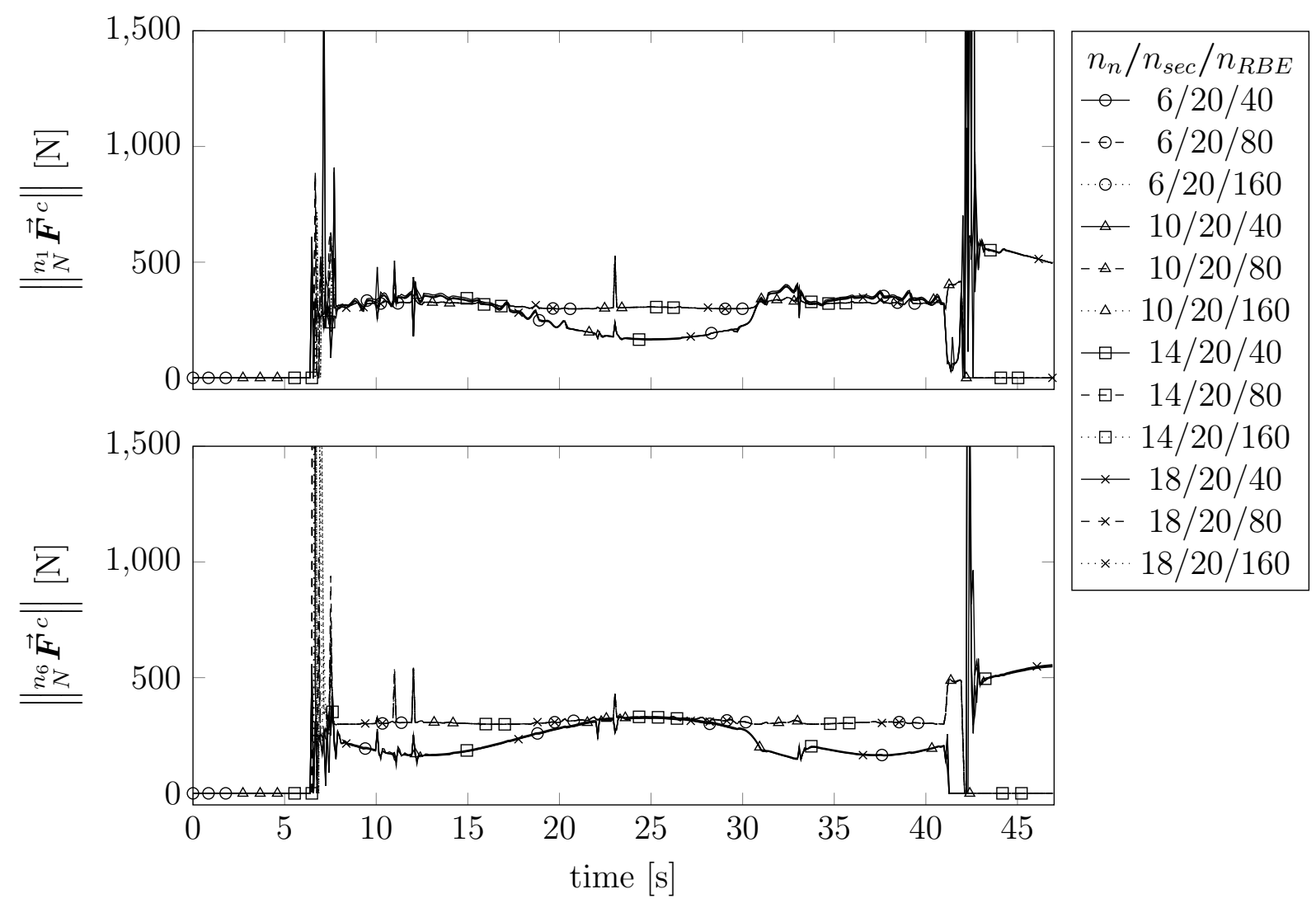

Figure 6.41: Case 8 Contact force magnitudes at each contact node. 


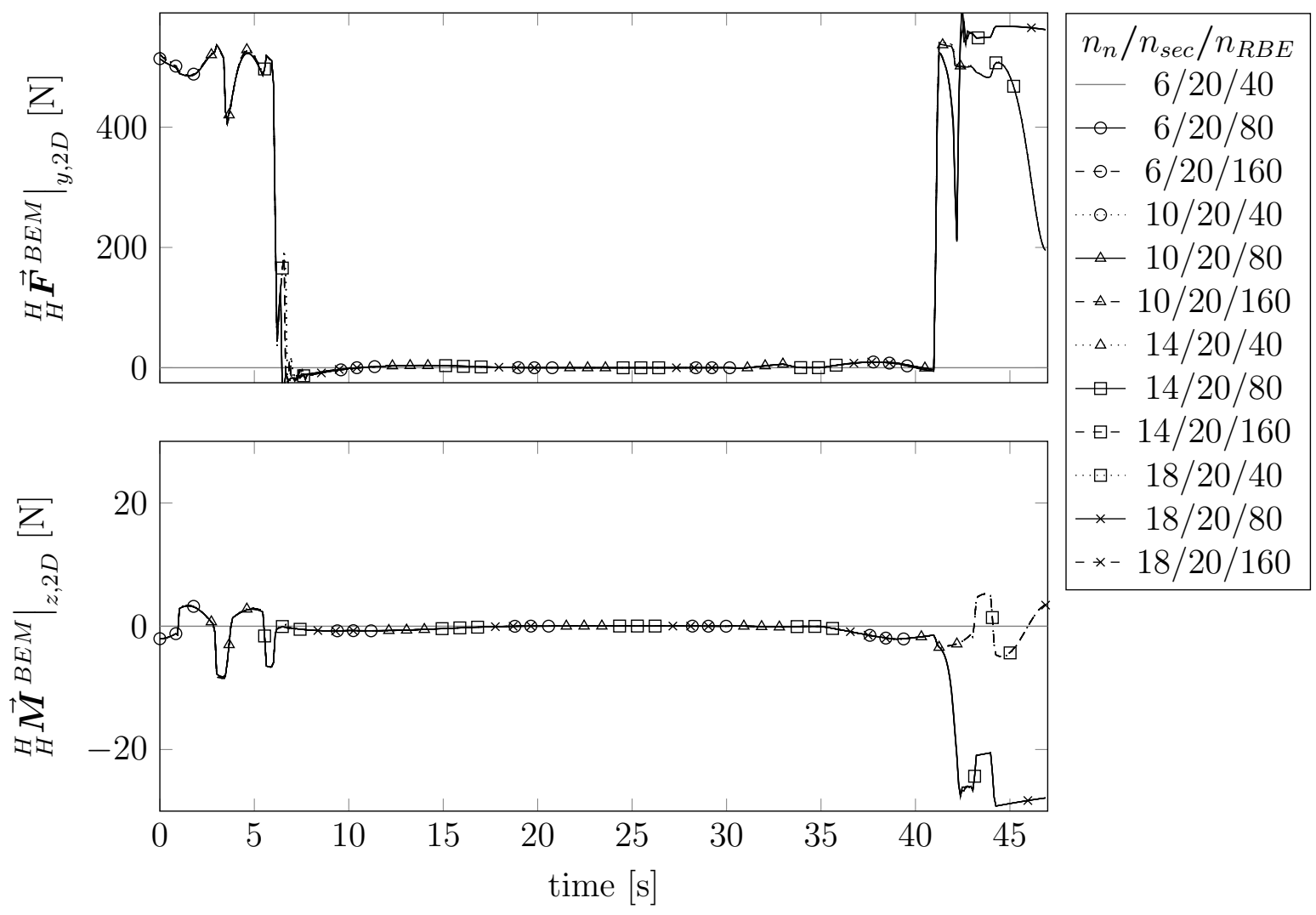

Figure 6.42: Case 8 Rotor disc force magnitudes. 


\subsection{General Remarks}

Test Cases 1 through 8 extensively analyse the models derived in Chapter 4 for proper integration into SRAMSS. Even so, there are key aspects which have not been explicitly tested in any test case, yet their proper functioning is required nonetheless.

The development of the equations of motion in Chapter 3 through to system assembly in Chapter 5, follows a particular variation of Kane's method for generating the equations of motion. Typically, an active force vector is assembled directly for each body in the system. In the case of the DFE model in SRAMSS, this would require that an active force vector be developed for each DFE node with consideration for the internal forces generated by other DFE nodes attached to it. However, the development presented in this work independently generates, then assembles the DFE model into the general system. This leaves the active force vector acting at each node to the assembly of externally-applied forces. In a system where a large body has flexible and rigid elements such as SRAMSS, an independently-generated DFE model allows direct use of any method for DFE model generation since only the $[\boldsymbol{M}],[\boldsymbol{C}]$, and $[\boldsymbol{K}]$ matrices need to be supplied for the system. Moreover, it enables infinitely-configurable and substitutable DFE models. Though, independent DFE matrix generation requires a way to interface the DFE model to the rigid components of the body. Section 4.4.1 and Section 5.1 present the methodology used to integrate the DFE model into the complete system. No test case in this chapter explicitly tests this interface model. An example which verifies this method and highlights the proper integration of the DFE model is the RSD manoeuvring simulation of Subcase 7.2. Even while the RSD is in motion, all distance magnitudes remain constant in Figure 6.35 when secured. This indicates that the rigid airframe and comparativelyflexible DFE-modelled landing gear are moving as a single body. In all other cases, the airframe and DFE responses suggest that the DFE model remains attached to the airframe in the expected way.

Specifically for the DFE model, a full-DoF 2D beam element is used. It can be argued that a simple beam element is sufficient since the axial compression of the element is negligible compared to the deflections in shear and bending. As it relates to simulation performance, time-complexity is exponential with respect to the size of the system, therefore reducing the number of DoFs would increase simulation performance. For analysis capabilities, retaining the axial DoFs would allow direct evaluation of buckling loads during simulations. This could be beneficial to the pre- 
liminary design of skid-landing gear, which often use long slender beams, suited to maritime shipboard operations.

At the observable level, the contact dynamics model has been verified to generate appropriately-oriented dynamic responses in every test case. It is required to work properly as all cases depend on ship-aircraft interaction to varying degrees. The most significant being Case 8 where all four defined polygons are in motion and the aircraft interacts with multiple polygons simultaneously. At a more fundamental level, the underlying contact detection algorithm presented in Section 5.2.2 is required to work correctly. In all test cases, surface contact was correctly detected which allowed for appropriate orientation of the dynamic response.

Furthermore, the contact dynamics formulas and algorithms used to express the response of a given surface on a contact node were developed with respect to a nonspecific generalized surface defined by two vertices in the two-dimensional Cartesian plane. The general case allows for infinitely configurable convex polygons to be defined for the system. This capability was exploited to represent major simulation elements as physical bodies. The ship deck, RSD deck, and wedge clamps were all successfully modelled using defined polygons. Additionally, the defined polygons could be considered for solution in the equations of motion, provided kinematic development to the appropriate frame of reference and force vectors are compiled. Such considerations could allow the simulation of ship response due to aircraft interaction, or even the RSD deck response in 'power-failure' or 'damaged' simulation scenarios.

Concerning the BEM model, Sub-case 6.5 indicates that the BEM model requires refinement to generate more accurate thrust profiles. Even so, the question of required BEM model fidelity remains: what accuracy is required to generate accurate aircraft response. In the case of SRAMSS, the BEM model is used to model a single rotor disc force and moment vector acting on the airframe. When the three-dimensional case of SRAMSS is implemented, the minimum accuracy required for the BEM model would only require that the simulated aircraft response matches experimental data. Such a model might not need to make considerations for more complex rotor disc phenomena such as downwash, blade wake, and vortex generation.

With respect to simulation performance, an ODE solver with root-finding capabilities is the required minimum as the contact detection algorithm leverages root functions. The choice of the DLSODAR solver also has the benefit of automatic selection of integration method based on system numerical stiffness. Simulation phases 
where the aircraft was in flight benefited from the non-stiff method as computational time fell well below the real-time threshold. While in contact, the stiff method allowed for state propagation in reasonable time.

For the most complex verification test case, Case 8 "18/20/160", Appendix E.1 lists the ratio of propagated simulation time to CPU computation time for the 6 to 10 seconds simulated time range. The range corresponds to the landing and aircraft securing phase. Values greater than 1 indicate faster than real-time solution propagations. Where the ratio is equal to 'inf', the CPU computation time was exceedingly small such that the Intel ${ }^{\circledR}$ Fortran's intrinsic 'CPU_TIME()' function considered $t$ and $t+\Delta t_{s o l}$ to be the same time. Drops in the ratio mostly occur where there is a non-smooth transition in any of the prescribed time-varying values or contact states. A smoothing function to remedy the transition points in prescribed values could yield significant simulation performance improvements. 


\section{Chapter 7}

\section{Conclusion}

As it pertains to the gap in dynamic interface analysis capabilities identified in Table 1.1, this work, the planar case of SRAMSS has demonstrated the ability to address the limitations presented by the current state-of-the-art. The kinematic and kinetic developments presented in Chapters 3 and 4 render a system formulation which, through extensive verification and validation of SRAMSS in Chapter 6, has shown extensive capabilities with respect to the simulation of securing and manoeuvring of skid-equipped rotary wing aircraft undergoing shipboard operations. The combination of rigid and flexible dynamics, oriented contact dynamics, and three-dimensional aerodynamics allows SRAMSS to include a wide range of phenomena and generate a detailed aircraft response for all phases of shipboard operations.

\subsection{Completed Work}

Resulting from achieving the objectives framed in Section 1.5, the following conclusions regarding the planar case of SRAMSS are drawn:

- Through the identification of major requirements for the shipboard securing and manoeuvring of skid-equipped rotary wing aircraft, a 'wedge clamp' mobile securing platform was conceptualized. Evaluation of SRAMSS has shown this design to be an effective securing and manoeuvring device even under large ship movement, all while being a passive member completely contained on the ship.

- A versatile contact dynamics model coupled with prescribed ship motion and aerodynamic aircraft control has enabled the complete simulation of recovery flight, securing, manoeuvring, and launch phases of shipboard helicopter oper- 
ations. The contact dynamics model is a novel application of the Separation Axis Theorem for collision detection. the SAT has been leveraged to both detect collision and generate an appropriately-oriented dynamic response. The contact dynamics model was verified to provide appropriately-oriented dynamic responses under the complex contact conditions present for stiff skid-type landing gear. Furthermore, the model was sufficiently generalized that it can incorporate any shaped convex polygons, which could be leveraged to model current and future securing and manoeuvring systems.

- The novel variant on Kane's method presented in this work has allowed the DFE model to be generated independently into the equations of motion from the rest of the system. Provided the correct DFE element mapping and element properties, the method enables the capability to model a wide range of skid and other rigid landing gear configurations. Not only this, the formulations and developed in this work to merge rigid and flexible body dynamics in Kane's method have applications beyond the scope of this work. It has shown the methodical integration of rigid and flexible components for a given body. This capability permits granularity in the selection of rigid and flexible elements when developing any complex dynamical system.

- The aerodynamic models developed in this work form a comprehensive foundation for capturing the aerodynamic phenomena present in shipboard operations. The aerodynamic body drag force model addressed the forces induced by the apparent wind over the ship deck, while the rotor disc BEMT model captured controlled rotor disc thrust and induced forces in apparent wind. The two aerodynamic models integrated into SRAMSS were verified to work correctly. Yet the BEM requires further development to provide more accurate results. Nevertheless, the BEM limits the dependency on manufacturer-provided and protected look-up tables, and is not limited by the range of data provided in these tables.

- The planar case of SRAMSS captures a wide variety of phenomena present in shipboard operations. The verification of the models implemented to capture these phenomena indicates that the simulation architecture and system formulation used for the planar case should form an appropriate base for the fully-spatial version of SRAMSS. The equations presented are sufficiently general that the development of the additional DoFs in the augmentation to three dimensions 
requires minimal re-derivation of equations. In certain areas, formula derivations become simpler as the kinematic-dynamic context of skew matrices is not needed. In other areas, additional formulae would be required for the new DoFs, such as the torsional stiffness in the DFE elements. High-level recommendations for the augmentation to three dimensions are presented in Section 7.2.

- The simulation package has been successfully written in the Intel ${ }^{\circledR}$ Fortran language. Step-wise simulation run-time has indicated the potential for simulations which run faster than real-time-a desirable trait for simulations. As such, SRAMSS could form the dynamical response component of real-time simulations for training pilots and deck crews.

\subsection{Recommendations}

The SRAMSS simulation package has the potential to provide a comprehensive simulation environment for skid-equipped rotary-wing aircraft undergoing shipboard manoeuvres. These capabilities are ultimately necessary in the study of shipboard UAS operations due to their emerging role in maritime shipboard environments. While this work develops and discusses the 2D verification of SRAMSS, the governing equations and algorithms contained within the SRAMSS simulation package are written to be easily extended to a three-dimensional simulation environment. Recommendations for the development of the three-dimensional version of SRAMSS follow below.

- The architecture of SRAMSS, including but not limited to the generation of the equations of motion, DFE model and system assembly, choice of ODE solver, collision detection, and contact dynamics algorithms should be re-used for the fully-spatial SRAMSS simulation package as this work has been verified to function correctly in comprehensive simulation test cases.

- Due to the high manoeuvrability of UAS, Euler parameters should be used to describe orientation and transformation matrix construction, rather than the currently-implemented Euler angles, to avoid potential singularities in the transformation matrices.

- Analogous to the left-normal unit vectors describing a surface in two dimensions, polygon surface normal unit vectors can be defined by the normal vector describing the surface plane in three dimensions. Similar to the use of the surface origin vertex as the maximum of projected vertices along a surface normal 
in $2 \mathrm{D}$, the first vertex in a set of vertices defining a surface can be used in 3D.

- The DFE model elements should be upgraded to 12-DoF general beam elements to capture behaviour in all degrees of freedom.

- The aerodynamics models used in the planar case have already been developed in three dimensions, and thus can be directly re-used in the full version of SRAMSS.

- Simulation statistics show a degradation in performance around simulation times where time-varying prescribed inputs undergo instantaneous change. A smoothing function to pre-process these regions could increase simulation performance.

- The inclusion of the third dimension approximately doubles the number of generalized coordinates and velocities. The increase in size of the system to be solved will considerably affect simulation performance. Investigation of code optimizations and executable parallelization will be necessary to exceed or simply retain performance at a level similar to the planar version of SRAMSS, excluding the consideration of faster computers.

- SRAMSS and SSMASH follow similar developments of the equations of motion by using Kane's method. SRAMSS's more sophisticated contact dynamics model and comprehensive aerodynamics model could form the basis for a universal dynamic interface analysis package. Integration of the wheeled-landing gear developments in SSMASH would allow the package to comprehensively evaluate aircraft regardless of landing gear type. It also presents the possibility of modelling hybrid wheeled-rigid landing gear.

- As it stands, the response of the aircraft to ship motion is of bigger concern than the opposite in regards to dynamic interface analysis. The partial velocity matrices could be reduced in size to eliminate the columns associated with prescribed ship deck, RSD deck, and wedge clamp motions. As a result, simulation performance would increase through a reduction of array size, and subsequent array manipulation time in 'XddotF.f90'.

- Nevertheless, for other applications, the ship and RSD deck response might be significant. Since the defined polygons form part of the partial velocity matrices and the polygon velocities have been developed, minimal additional derivations are required for inclusion of their response in simulations. Design failure mode and effect analysis (DFMEA) and extreme edge-case simulations would benefit 
from ship and RSD response inclusion.

\subsection{Future Work}

The planar case of SRAMSS is an intermediate step in the development of the fullyspatial version of SRAMSS. This work has demonstrated that the system formulation works as intended. Accordingly, work will be undertaken to expand the system formulation into the third dimension. There, the fully-spatial version of SRAMSS will be validated against experimental dynamic interface data collected in aerodynamic test facilities such as Carleton University's state-of-the-art WInDLab wind tunnel equipped with a 6-DoF motion platform. 


\section{References}

[1] F. Cracknell, "LAMPS III Recovery Assist, Securing and Traversing (RAST) System," DAF Indal Ltd., Mississauga, ON, Canada, SAE Technical Paper Series 811080, 1981.

[2] (2015) Naval Handling Systems. Curtiss-Wright Indal. [Online]. Available: https://s2.q4cdn.com/767595508/files/doc_downloads/indal/modeling/INDALBrochure.pdf [Accessed: 2020-06-28]

[3] J. Blackwell and R. A. Feik, "A Mathematical Model of the On-deck Helicopter/ship Dynamic Interface," Aeronautical Research Laboratory, Melbourne, Australia, Tech. Rep. ARL-AERO-TM-405, 1989.

[4] A. R. Tadros and R. G. Langlois, "Securing and Handling Helicopters on Board Ships, Essential Factors for Meeting Safety and Operational Requirements," Indal Technologies Inc., Missisauga, ON, Canada, Tech. Rep. [Online]. Available: https://www.cw-ems.com/indal/products/helicopter-securing-andtraversing/default.aspx

[5] D. R. Linn and R. G. Langlois, "Development and Experimental Validation of a Shipboard Helicopter On-Deck Maneuvering Simulation," Journal of Aircraft, vol. 43, no. 4, pp. 895-906, 2006.

[6] D. R. Langlois, D. Z. H. Zhu, and M. LaRosa, "Modelling and Simulation of Skid-equipped Shipboard Rotorcraft," Curtiss-Wright - INDAL Technologies, Mississauga, ON, Canada, Tech. Rep., 2015. [Online]. Available: https://www.cw-ems.com/indal/products/helicopter-securing-andtraversing/default.aspx 
[7] A. J. Garcia and G. Barakos, "Accurate Predictions of Rotor Hover Performance at Low and High Disc Loadings," Journal of Aircraft, vol. 55, no. 1, pp. 89-110, January 2018.

[8] D. T. Balch, A. Saccullo, and T. W. Sheehy, "Experimental study of Main Rotor Tip Geometry and Tail rotor Interactions in Hover - Volume I," Ames Research Center, Moffett Field, CA, Tech. Rep. NASA CR-166485, June 1983.

[9] R. Ernst and B. Ferrier, "Fire Scout Launch and Recovery Considerations in Unexpected Ship Roll Motion Conditions," Naval Engineers Journal, vol. 129, no. 4, pp. 87-98(12), December 2017.

[10] C. N. Manley, "Armed Drones for the Royal Canadian Navys," Canadian Forces College, Toronto, ON, Canada, Tech. Rep., 2017. [Online]. Available: https://www.cfc.forces.gc.ca/259/290/405/305/manley.pdf

[11] H. Kumar and T. Husseini. (2019) QinetiQ to Supply Unmanned Aircraft Systems to Canadian Armed Forces. [Online]. Available: https://www.navaltechnology.com/news/canadian-navy-drones-qinetiq/ [Accessed: 2020-06-27]

[12] Heligrid Helicopter Landing System. Cramm HLS. [Online]. Available: https://pdf.nauticexpo.com/pdf/cramm-yachting-systemsbv/heligrid/22833-41191.html [Accessed: 2020-06-28]

[13] (2016) Helicopter and UAV Flight Deck Securing and Handling Devices. Naval Group. [Online]. Available: https://www.naval-group.com/wp-content/uploads/2017/01/helicopterand-uav-flight-deck-securing-and-handling-devices.pdf [Accessed: 2020-06-28]

[14] R. T. Schmitke and B. W. Whitten, "SHIPMO - A FORTRAN Program to Predict Ship Motions in Waves (1981)," 1981, DREA Technical Memorandum $81 / \mathrm{C}$.

[15] G. L. Loeff and F. van Walree, "ITI Developed New Time Domain Ship Motion Program for High Sea State Conditions (SPLAShMo)," February 2006, naval Forces.

[16] R. G. Langlois, M. LaRosa, and D. A. R. Tadros, "Development, Validation, and Application of the Dynaface ${ }^{\circledR}$ Helicopter/Ship Dynamic Interface Simulation Software Package," vol. 35, San Diego, CA, 2003, edited by V. W. Ingalls. 
[17] (2016) Seakeeping Prediction Software. BMT. [Online]. Available: https://www.bmt.org/media/3646/shipmopc_seakeeping_prediction.pdf [Accessed: 2020-08-31]

[18] G. L. Loeff and F. van Walree, "Validation of Maneuvering Prediction Capabilities of FREDYN, FREDYN version 9.6," MARIN, Tech. Rep. No. 08883-45CPM, February 2006.

[19] A. R. Feldman and R. G. Langlois, "Development of an Experimental Aircraft/ship Dynamic Interface Analysis Motion Facility," in In Proc. 7th Canadian Marine Hydromechanics and Structures Conference, Halifax, NS, Canada, September 2005.

[20] —_, "Autonomous Straightening and Traversing of Shipboard Helicopters," Journal of Field Robotics, vol. 23, no. 2, pp. 123-139, February 2006.

[21] M. J. Léveillé and R. G. Langlois, "Spacial Modelling and Simulation of Ondeck Helicopter Securing and Manoeuvring," in Proceedings of the 70th Annual Forum of the American Helicopter Society, Montreal, QC, Canada, May 2014, pp. $740-749$.

[22] D. R. G. Langlois and D. A. R. Tadros, "User's Manual for the Aircraft/Ship Dynamic Interface Simulation Dynaface ${ }^{\circledR}$ Release 5.0.” Indal Technologies Inc, Mississauga, ON, Canada, Tech. Rep. ITI Report No. 99/419, January 1998.

[23] S. R. Borneman, J. McCallum, and M. LaRosa, "Extending UAV Operating Envelopes," Curtiss-Wright - INDAL Technologies, Mississauga, ON, Canada, Tech. Rep., 2015. [Online]. Available: https://www.cwems.com/indal/products/helicopter-securing-and-traversing/default.aspx

[24] R. G. Lee and S. J. Zan, "Unsteady Aerodynamic Loads On a Helicopter Fuselage In a Ship Air Wake," Montreal, QC, Canada, May 2002.

[25] S. J. Zan, "Experimental Determination of Rotor Thrust in a Ship Airwake," Journal of the American Helicopter Society, vol. 47, no. 2, pp. 100-118, April 2002.

[26] E. Alpman, L. N. Long, D. O. Bridges, and J. F. Horn, "Fully-coupled Simulations of the Rotorcraft/ship Dynamic Interface," American Helicopter Society 63rd Annual Forum, Virginia Beach, VA, U.S.A., May 2007. 
[27] A. S. Wall, S. J. Zan, R. G. Langlois, and F. F. Afagh, "Correlated Turbulence Modelling: An Advancing Fourier Series Method," Journal of Wind Engineering and Industrial Aerodynamics, vol. 123, no. Part A, pp. 155-162, December 2013.

[28] A. S. Wall, R. G. Langlois, F. F. Afagh, and S. J. Zan, "Modelling Helicopter Blade Sailing: Contributions From Ship Motion and Unsteady Airwake to Blade Dynamic Response," Toronto, Canada, April 2007.

[29] F. Khouli, A. S. Wall, F. F. Afagh, and R. G. Langlois, "Influence of Ship Motion On the Aeroelastic Response of a Froude-scaled Maritime Rotor System," vol. 54, January 2007, pp. 170-181.

[30] RotorLib FDM for $C++$, RTDynamics, Rosenheim, Germany. [Online]. Available: http://www.rtdynamics.com/products-services/simulation-andtraining/rotorlib-fdm-for-c/?doing_wp_cron=1593700957.7624619007110595703125

[31] S. Kockara, T. Halic, K. Iqbal, C. Bayrak, and R. Rowe, "Collision detection: A survey," in 2007 IEEE International Conference on Systems, Man and Cybernetics, 2007, pp. 4046-4051.

[32] M. Figueiredo, L. Marcelino, and T. Fernando, "A Survey on Collision Detection Techniques for Virtual Environments," 2002.

[33] C. Liang and X. Liu, "The Research of Collision Detection Algorithm Based on Separating axis Theorem," International Journal of Science, vol. 2, no. 10, pp. 110-114, 2015.

[34] S. Gottschalk, "Separating Axis Theorem," Department of Computer Science, UNC Chapel Hill, Tech. Rep. TR96-024, 1996.

[35] G. Gilardi and I. Sharf, "Literature Survey of Contact Dynamics Modelling," Mechanism and Machine Theory, vol. 37, no. 10, pp. 1213 - 1239, 2002.

[36] V. Lampaert, J. Swevers, and F. Al-Bender, "Experimental Comparison of Different Friction Models for Accurate Low-velocity Tracking," 2002.

[37] Y. Liu, J. Li, Z. Zhang, X. Hu, and W. Zhang, "Experimental Comparison of Five Friction Models on the Same Test-bed of the Micro Stick-slip Motion System," Mechanical Sciences, vol. 6, pp. 15-28, March 2015. 
[38] F. Marques, P. Flores, J. C. P. Claro, and H. M. Lankarani, "A Survey and Comparison of Several Friction Force Models for Dynamic Analysis of Multibody Mechanical Systems," Nonlinear Dynamics, vol. 86, no. 3, pp. 1407-1443, November 2016.

[39] E. Pennestrì, V. Rossi, P. Salvini, and P. P. Valentini, "Review and Comparison of Dry Friction Force Models," Nonlinear Dynamics, vol. 83, no. 4, pp. 17851801, March 2016.

[40] C. C. de Wit, H. Olsson, K. J. Astrom, and P. Lishinsky, "A New Model for Control of Systems with Friction," IEEE Transactions On Automatic Controls, vol. 40, no. 3, pp. 418-425, March 1995.

[41] P. R. Dahl, "A Solid Friction Model," The Aerospace Corporation, El Segundo, CA, Tech. Rep. SAMSO-TR-77-131, 1968.

[42] J. Swevers, F. Al-Bender, C. G. Ganseman, and T. Prajogo, "An Integrated Friction Model Structure with Improved Pre-sliding Behavior for Accurate Friction Compensation," IEEE Transactions On Automatic Control, vol. 45, no. 4, pp. 675-686, 2000.

[43] W. Z. Stepniewski, "Basic Theories of Rotor Aerodynamics (With Applications to Helicopters)," Boeing Vertol Company, Philadelphia, PA, Tech. Rep. N7922039, January 1979.

[44] D. V. Leza, "Development of A Blade Element Method for CFD Simulations of Helicopters Using the Actuator Disc Approach," Master's thesis, Delft University of Technology, Delft, 2018.

[45] M. J. Léveillé, "Development of a Spacial Dynamic Handling and Securing Model for Shipboard Helicopters," Master's thesis, Carleton University, Ottawa, ON, Canada, 2013.

[46] P. E. Nikravesh, Computer-Aided Analysis of Mechanical Systems. Englewood Cliffs, NJ: Prentice Hall, 1988.

[47] T. R. Kane, P. W. Likins, , and D. A. Levinson, Spacecraft Dynamics. New York: McGraw-Hill, 1983. 
[48] A. Purushotham and J. Anjeneyulu, "Kane's Method for Robotic Arm Dynamics: a Novel Approach," IOSR Journal of Mechanical and Civil Engineering (IOSRJMCE), vol. 6, no. 4, pp. 7-13, May-June 2013.

[49] E. Stoneking, "Implementation of Kane's Method for a Spacecraft Composed of Multiple Rigid Bodies," August 2013.

[50] K.-J. Bathe, Finite Element Procedures. New Jersey: Prentice Hall, 1996, p. 768.

[51] R. D. Cook, Finite Element Modelling for Stress Analysis. Canada: Wiley, 1995, pp. 230-232.

[52] B. W. McCormick, Aerodynamics, Aeronautics, and Flight Mechanics. Wiley, August 1979, p. 196.

[53] R. L. Smith, "Closed-form Equations for the Lift, Drag, and Pitching-Moment Coefficients of Airfoil Sections in Subsonic Flow," Ames Research Center, Moffett Field, CA, Tech. Rep. NASA-TM-78492, August 1978.

[54] B. Stellato, S. Ober-Blöbaum, and P. Goulart, 2017.

[55] A. C. Hindmarsh, ODEPACK.f, Center for Applied Scientific Computing, L-561, Livermore, CA, February 2008, (DLSODAR Subroutine, 12 November 2003 Version). [Online]. Available: https://people.sc.fsu.edu/ jburkardt/f77_src/odepack/odepack.html

[56] — - "A Systematized Collection of ODE Solvers," IMACS Transactions on Scientific Computation, vol. 1, pp. 55-64, 1983, r. S. Stepleman et al. edits.

[57] L. R. Petzold, "Automatic Selection of Methods for Solving Stiff and Nonstiff Systems of Ordinary Differential Equations," SIAM Journal on Scientific and Statistical Computing, vol. 4, no. 1, pp. 136-148, 1983.

[58] K. L. Hiebert and L. F. Shampine, "Implicitly Defined Output Points for Solutions of ODEs," Sandia Labs, Albuquerque, NM, Tech. Rep. SAND-80-0180, February 1980.

[59] (2020) Airfoil Tools. [Online]. Available: http://airfoiltools.com/index [Accessed: 2020-08-04] 
[60] J. J. Totah, "A Critical Assesment of UH-60 Main Rotor Blade Airfoil Data," Ames Research Center, Moffett Field, CA, Tech. Rep. NASA-TM-103985.

[61] C. N. Keys, "Performance Prediction of Helicopters," Boeing Vertol Company, Philadelphia, PA, Tech. Rep. NASA-CR-3083, January 1979. 


\section{Appendix A}

\section{SRAMSS I/O File Structure}

This appendix outlines the structure of the input and output files for SRAMSS. The input files include the complete description of Initial conditions, aircraft parameters, prescribed motions, and simulation parameters required. The output Files contain the time-stamped raw data calculated by SRAMSS.

\section{A.1 Input Files}

\section{Simulation.inp}

Line 1: $t_{\text {start }} t_{\text {end }}$ simulation time start and end $[\mathrm{s}]$

Line 2: $\Delta t_{\text {sol }}$ integrator default solution time-step $[\mathrm{s}]$

Line 3: $t_{\text {mult }}$ Multiplier for number of solution vectors stored before print to file $\left(t_{\text {end }}-\right.$ $\left.t_{\text {start }}\right) / \Delta t_{\text {sol }} t_{\text {mult }}$

Line 4: $N_{P}$ Number of polygons in simulation environment

Line 5: $\mathbb{Z} \quad$ DFE $[\boldsymbol{M}]$ assembly method [0=SRAMSS automatic generation, 1=Read-in]

Line 6: $\mathbb{Z} \quad$ DFE $[\boldsymbol{C}]$ assembly method [0=SRAMSS automatic generation, $1=$ Read-in]

Line 7: $\mathbb{Z} \quad$ DFE $[\boldsymbol{K}]$ assembly method [0=SRAMSS automatic generation, $1=$ Read-in]

Line 8: $\mathbb{Z} \quad$ DLSODAR: ITOL error control option.

Line 9: $\mathbb{R} \quad$ DLSODAR: RTOL relative error tolerance

Line 10: $\mathbb{R}$ DLSODAR: ATOL absolute error tolerance 
Line 11: $\mathbb{Z}$ DLSODAR: ITASK integration task type

Line 12: $\mathbb{Z}$ DLSODAR: ISTATE $==1$ for initialization

Line 13: $\mathbb{Z} \quad$ DLSODAR: JT Jacobian type flag

Line 14: $\mathbb{Z}$ DLSODAR: IOPT optional inputs flag, if any of RWORK or IWORK are set to 0.d0 or 0, DLSODAR will use default value

Line 15: $\mathbb{R}$ DLSODAR: RWORK(5) if IOPT $==1$, H0 step size attempted on first step

Line 16: $\mathbb{R}$ DLSODAR: RWORK(6) if IOPT $==1$, HMAX the max absolute step size allowed

Line 17: $\mathbb{R}$ DLSODAR: $\operatorname{RWORK}(7)$ if IOPT $==1$, HMIN the min absolute step size allowed

Line 18: $\mathbb{Z}$ DLSODAR: $\operatorname{IWORK}(5)$ if IOPT $==1$, Flag to generate extra printing at method switches. [IXPR $=0$ extra printing, IXPR $=1$ print data on each switch]

Line 19: $\mathbb{Z}$ DLSODAR: IWORK(6) if IOPT $==1$, Max number of internal solver steps

Line 20: $\mathbb{Z}$ DLSODAR: $\operatorname{IWORK}(7)$ if IOPT $==1$, Maximum number of messages printed per problem.

Line 21: $\mathbb{Z}$ DLSODAR: $\operatorname{IWORK}(8)$ if IOPT $==1$, The maximum order to be allowed for the nonstiff (Adams) method. default value is 12, $[\leq 12]$.

Line 22: $\mathbb{Z}$ DLSODAR: $\operatorname{IWORK}(9)$ if IOPT $==1$, The maximum order to be allowed for the stiff (BDF) method. The default value is $5,[\leq 5]$.

Line 23: $\mathbb{Z}$ Print $\Delta t_{\text {sol }}$ Solver stats $[0=$ no stats, $1=$ time propagation stats, $2=$ full solution stats]

\section{Output_options.inp}

Line 1: $\mathbb{Z} \quad$ Generalized states solution output file $[1=$ yes, $0=$ no $]$

Line 2: $\mathbb{Z} \quad$ Simulation stats

Line 3: $\mathbb{Z} \quad$ Ship polygon output file

Line 4: $\mathbb{Z} \quad$ RSD polygon output file 
Line 5: $\mathbb{Z} \quad$ Left RSD clamp output file

Line 6: $\mathbb{Z} \quad$ Right RSD clamp output file

Line 7: $\mathbb{Z} \quad$ Ship OCP solution output file

Line 8: $\mathbb{Z} \quad$ RSD OCP solution output file

Line 9: $\mathbb{Z} \quad$ Left RSD clamp OCP output file

Line 10: $\mathbb{Z}$ right RSD clamp OCP output file

Line 11: $\mathbb{Z}$ Contact forces vector on $n_{j_{c}}$ in $N$ output file

Line 12: $\mathbb{Z}$ Induced thrust vector on $H$ in $H$ output file

\section{ICs.inp}

Line i: $\quad \boldsymbol{u}_{i} \mathbb{Z}$ Initial condition of generalized speed $u_{i}[\mathrm{~m} / \mathrm{s}]$, and generalized speed type $u_{i, \text { type }}[0=$ free, $1=$ contact, $2=$ fixed, $3=$ prescribed $]$. repeated line through all $u$

Line i: $\quad \boldsymbol{q}_{i} \mathbb{Z}$ Initial condition of generalized coordinate $q_{i}[\mathrm{~m}]$, and generalized coordinate type $q_{i, t y p e}[0=$ free, $1=$ contact, $2=$ fixed, $3=$ prescribed]. repeated line through all $q$

\section{Properties_helo.inp}

Line 1: $m_{H} \quad$ Airframe mass $[\mathrm{kg}]$

Line 2: $\quad I_{H} \quad$ Airframe moment of Inertia $\left[\mathrm{kg} \mathrm{m}^{2}\right]$

Line 3: $\quad N_{d} \quad$ Number of external dampers

Line 4: $\left.{ }_{H}^{H} \overrightarrow{\boldsymbol{r}}^{M_{1}}\right|_{x}, \ldots,\left.{ }_{H}^{H} \overrightarrow{\boldsymbol{r}}^{M_{N_{d}}}\right|_{x} \quad$ Winglet external shock mount x-position [m]

Line 5: $\left.{ }_{H}^{H} \overrightarrow{\boldsymbol{r}}^{M_{1}}\right|_{y}, \ldots,\left.{ }_{H}^{H} \overrightarrow{\boldsymbol{r}}^{M_{N_{d}}}\right|_{y} \quad$ Winglet external shock mount y-position [m]

Line 6: $\left.{ }_{H}^{H} \overrightarrow{\boldsymbol{v}}^{M_{1}}\right|_{x}, \ldots,\left.{ }_{H}^{H} \overrightarrow{\boldsymbol{v}}^{M_{N_{d}}}\right|_{x} \quad$ Winglet external shock mount x-velocity $[\mathrm{m} / \mathrm{s}]$

Line 7: $\left.{ }_{H}^{H} \overrightarrow{\boldsymbol{v}}^{M_{1}}\right|_{y}, \ldots,\left.{ }_{H}^{H} \overrightarrow{\boldsymbol{v}}^{M_{N_{d}}}\right|_{y} \quad$ Winglet external shock mount y-velocity $[\mathrm{m} / \mathrm{s}]$

Line 8: $n_{j_{1}}, \ldots, n_{j_{d}}$ DFE node mount number

Line 9: $\quad \Delta_{d_{1}, 0}, \ldots, \Delta_{d_{n}, 0} \quad$ Underformed discrete damper lengths [m] 
Line 10: $K_{d} \quad$ Spring constant for linear discrete damper $[\mathrm{N} / \mathrm{m}]$

Line 11: $C_{d}$ Damping constant for linear discrete damper $[\mathrm{Nm} / \mathrm{s}]$

Line 12: ${ }_{H}^{H} \overrightarrow{\boldsymbol{r}}^{C P} \quad$ Airframe Centre of pressure [m]

Line 13: $\boldsymbol{A}_{e q, x}$ Airframe equivalent flat plate area $\left[\mathrm{m}^{2}\right]$

Line 14: ${ }_{H}^{H} \overrightarrow{\boldsymbol{r}}^{R} \quad$ Rotor disc center $[\mathrm{m}]$

Line 15: $N_{b}$ Number of rotor blades

Line 16: $\quad R_{b} \quad$ Radius of rotor blade $[\mathrm{m}]$

Line 17: $\quad R_{i} \quad$ Inner effective blade radius as percentage of $R_{b}[\%]$

Line 18: $R_{o}$ Outer effective blade raidus as percentage of $R_{b}[\%]$

Line 19: $N_{e} \quad$ Number of blade elements

Line 20: $N_{\psi} \quad$ Number of rotor disc sectors

Line 21: $\mathbb{Z} \quad$ Number of data points for rotor blade profile $(r)$

Line 22: $\quad r$ Blade data point radii $[r=0$ to $r=R]$ limits of blade radius must be outside bounds set by $R_{i} R_{b}$ to $R_{o} R_{b}[\mathrm{~m}]$

Line 23: $c(r)$ Blade chord length for each blade data point radii [m]

Line 24: $\theta_{t}(r) \quad$ Blade twist angle @radius [rad]

Line 25: $t / c(r) \quad$ Blade thickness ratio [\%]

Line 26: $\frac{\mathrm{d} C_{L}}{\mathrm{~d} \alpha}(r) \quad$ Linear slope $C_{L}$ vs $\alpha\left[\operatorname{rad}^{-1}\right]$

Line 27: $C_{L, 0}(r)$ zero angle of attack $C_{L}$

Line 28: $\frac{\mathrm{d} C_{D}}{\mathrm{~d} \alpha}(r) \quad$ Linear slope $C_{D}$ vs $\alpha\left[\operatorname{rad}^{-1}\right]$

Line 29: $C_{D, 0}(r) \quad$ zero angle of attack $C_{D}$ 


\section{Properties_contact_mech.inp}

Line 1: $\quad K_{p}$ Penetration stiffness $[\mathrm{N} / \mathrm{m}]$

Line 2: $C_{p}$ Penetration damping $[\mathrm{Nm} / \mathrm{s}]$

Line 3: $\quad K_{f} \quad$ Friction stiffness coefficient $[\mathrm{N} / \mathrm{m}]$

Line 4: $\quad C_{f} \quad$ Friction damping coefficient $[\mathrm{Nm} / \mathrm{s}]$

Line 5: $\mu_{d}$ Dynamic friction coefficient

Line 6: $\mu_{s}$ Static Friction coefficient

Line 7: $v_{s} \quad$ Stribeck velocity threshold $[\mathrm{m} / \mathrm{s}]$

\section{Properties_FE_map.inp}

Line i: $e_{r}, n_{a}, n_{b}, E_{r}, I_{r}, A_{r}, R \quad r$ th DFE element properties: element no., node a, node b, E $[\mathrm{Pa}], \mathrm{I}\left[\mathrm{m}^{4}\right], \mathrm{xA}\left[\mathrm{m}^{2}\right]$, density $\left[\mathrm{kg} \mathrm{m}^{-3}\right]$. line repeated for all elements in DFE model.

\section{Properties_FE_IC.inp}

Line i: $\quad \boldsymbol{q}_{i} \mathbb{Z}$ Undeformed DFE generalized coordinate $\boldsymbol{q}_{i}[\mathrm{~m}]$, and generalized coordinate type $\boldsymbol{q}_{i, \text { type }}=[0=$ free, $1=$ contact, $2=$ fixed, $3=$ prescribed $]$. repeated line through to $\boldsymbol{q}_{18+3 N_{n}}$

\section{Properties_FE_*mat.inp}

Line i: $\left[*_{i}\right]$ Row i of optional read-in DFE matrix. $\left[{ }^{*}=\mathrm{M}\right.$ Properties_FE_Mmat.inp, $*=$ C Properties_FE_Cmat.inp, ${ }^{*}=$ K Properties_FE_Kmat.inp]. Repeated for all rows of DFE matrix

\section{Polygon_*.inp}

Line 1: $\left.\quad{ }_{*}^{*} \overrightarrow{\boldsymbol{r}}^{*_{1}}\right|_{x}, \ldots \quad,\left.{ }_{*}^{*} \overrightarrow{\boldsymbol{r}}^{*_{m}}\right|_{x}{ }^{*}$ polygon vertices x-positions [m], polygon designation $\left[{ }^{*}=\right.$ S Prescribed_ship.inp, ${ }^{*}=$ D Prescribed_rsd.inp $*=$ CL Prescribed_clampleft.inp, $*=$ CR Prescribed_clampright.inp] 
Line 2: $\left.{ }_{*}^{*} \overrightarrow{\boldsymbol{r}}^{*_{1}}\right|_{y}, \ldots \quad,\left.{ }_{*}^{*} \overrightarrow{\boldsymbol{r}}^{*_{m}}\right|_{y} *$ polygon vertices y-positions [m], polygon designation $\left[{ }^{*}=\mathrm{S}\right.$ ship deck, ${ }^{*}=\mathrm{D}$ RSD deck, ${ }^{*}=\mathrm{CL}$ left wedge clamp, ${ }^{*}=\mathrm{CR}$ right wedge clamp]

\section{Prescribed_*inp}

Line $t:{ }_{*-1}^{*-1} \overrightarrow{\boldsymbol{r}}^{*}$ At time $\mathrm{t}[\mathrm{s}]$, prescribed position of frame with respect to preceding frame in chain of frames $[\mathrm{m}] .{ }_{N}^{N} \overrightarrow{\boldsymbol{r}}^{S},{ }_{S}^{S} \overrightarrow{\boldsymbol{r}}^{D},{ }_{D}^{D} \overrightarrow{\boldsymbol{r}}^{C L},{ }_{D}^{D} \overrightarrow{\boldsymbol{r}}^{C R}$ in separate Prescribed_*.inp files.

\section{Prescribed_aero.inp}

Line $t: \quad t,{ }_{N}^{N} \overrightarrow{\boldsymbol{v}}^{W},{ }_{R}^{R} \overrightarrow{\boldsymbol{\omega}}^{R B E}, \theta_{\text {coll }}, \theta_{\text {cyc,max }}, \psi_{\max }$ At time $\mathrm{t}[\mathrm{s}]$, wind velocity $[\mathrm{m} / \mathrm{s}]$, rotor $\mathrm{rpm}[\mathrm{rad} / \mathrm{s}]$, collective pitch setting [rad], cyclical pitch setting [rad], maximum cyclic pitch azimuth [rad]. 


\section{A.2 Output Files}

\section{GEN_SOLUTION.out}

Line $t: \quad t, \boldsymbol{u}, \boldsymbol{q}$ Time-stamped vector of generalized speeds and positions.

\section{$\mathrm{POLY}_{-}^{*}$.out}

Line $t: \quad t,\left.{ }_{N}^{N} \overrightarrow{\boldsymbol{r}}^{* 1}\right|_{x},\left.{ }_{N}^{N} \overrightarrow{\boldsymbol{r}}^{* 1}\right|_{y}, \ldots,\left.{ }_{N}^{N} \overrightarrow{\boldsymbol{r}}^{*_{m}}\right|_{x},\left.{ }_{N}^{N} \overrightarrow{\boldsymbol{r}}^{*_{m}}\right|_{y} \quad$ Time-stamped polygon vertices relative to the inertial frame $[\mathrm{m}]$, polygon designation $\left[{ }^{*}=\mathrm{S}\right.$ POLY_SHIP.out, ${ }^{*}=\mathrm{D}$ POLY_RSD.out, ${ }^{*}=$ CL POLY_L_CLAMP.out, ${ }^{*}=$ CR POLY_R_CLAMP.out]

\section{$\mathrm{OCP}_{-}^{*}$.out}

Line $t: \quad t,\left.{ }_{N}^{*} \overrightarrow{\boldsymbol{o}}^{n_{j_{1}}}\right|_{x},\left.{ }_{N}^{*} \overrightarrow{\boldsymbol{\sigma}}^{n_{j_{1}}}\right|_{y}, \ldots,\left.{ }_{N}^{*} \overrightarrow{\boldsymbol{\sigma}}^{n_{j_{c}}}\right|_{x},\left.{ }_{N}^{*} \overrightarrow{\boldsymbol{\sigma}}^{n_{j_{c}}}\right|_{y} \quad$ Time-stamped polygon OCPs relative to the inertial frame[m], polygon designation $\left[{ }^{*}=\mathrm{S}\right.$ OCP_SHIP.out, ${ }^{*}=\mathrm{D}$ OCP_RSD.out, ${ }^{*}=$ CL OCP_L_CLAMP.out, ${ }^{*}=$ CR OCP_R_CLAMP.out]

\section{FORCE_CONTACT.out}

Line $t: \quad t,{ }_{N}^{n_{j_{1}}} \overrightarrow{\boldsymbol{F}}_{k}^{c}, \ldots,{ }_{N}^{n_{j_{c}}} \overrightarrow{\boldsymbol{F}}_{k}^{c} \quad$ Time-stamped total contact forces on all contact nodes, expressed in the inertial frame $[\mathrm{N}, \mathrm{N}, \mathrm{Nm}]$

\section{FORCE_INDUCED.out}

Line $t: \quad t,{ }_{H}^{H} \vec{F}^{B E M},{ }_{H}^{H} \overrightarrow{\boldsymbol{M}}^{B E M} \quad$ Time-stamped rotor disc thrust and rolling-moments [N, $\mathrm{N}, \mathrm{Nm}]$

\section{SIM_STATS.out}

Line $t: t, \mathbb{Z}, \mathbb{Z}, \mathbb{Z}, \mathbb{Z}, \mathbb{Z}, \mathbb{Z}, \mathbb{R}, \mathbb{R}, \mathbb{Z}$ Time-stamped simulation and DLSODAR.for statistics and simulation summary. time $[\mathrm{s}]$, XddotF.f90 evaluations $/ \Delta t_{\text {sol }}$, Xd$\operatorname{dotF.f90~evaluations/~} t$, XddotG.f90 evaluations/ $\Delta t_{\text {sol }}$, XddotG.f90 evaluations $/ t$, DLSODAR internal steps $/ \Delta t_{s o l}$, DLSODAR internal steps $/ t, t_{C P U} / \Delta t_{s o l}\left[\mathrm{~s}_{C P U}\right]$, $t_{C P U} / t\left[\mathrm{~s}_{C P U}\right], \# \Delta t_{s o l}$ 


\section{Appendix B}

\section{Case 6 Prescribed Aerodynamics}

\section{B.1 Case 6.2: Rotor Thrust without Wind}
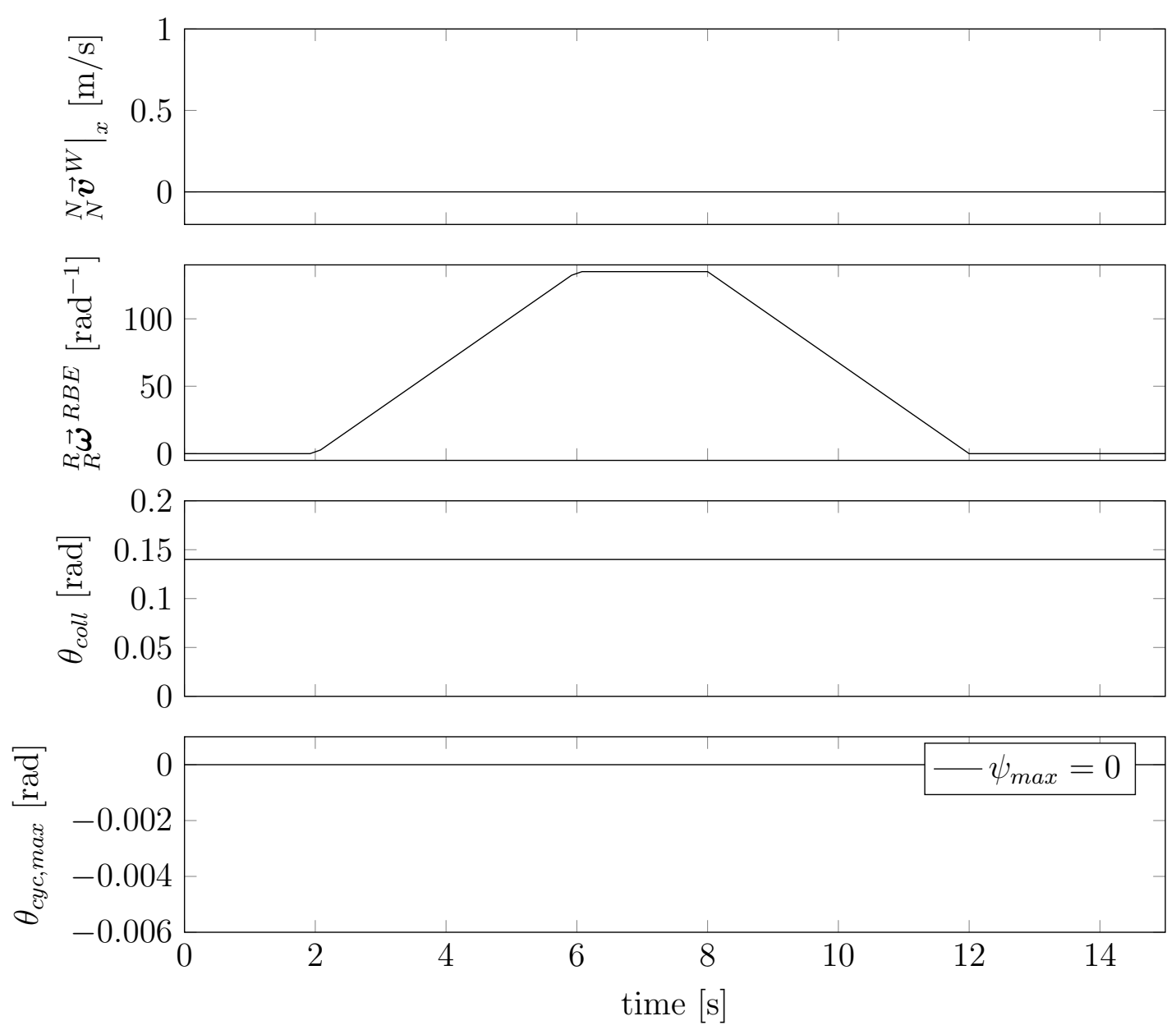

Figure B.1: Case 6.2 Prescribed aerodynamic values. 


\section{B.2 Case 6.3: Rotor Thrust with Head-wind}
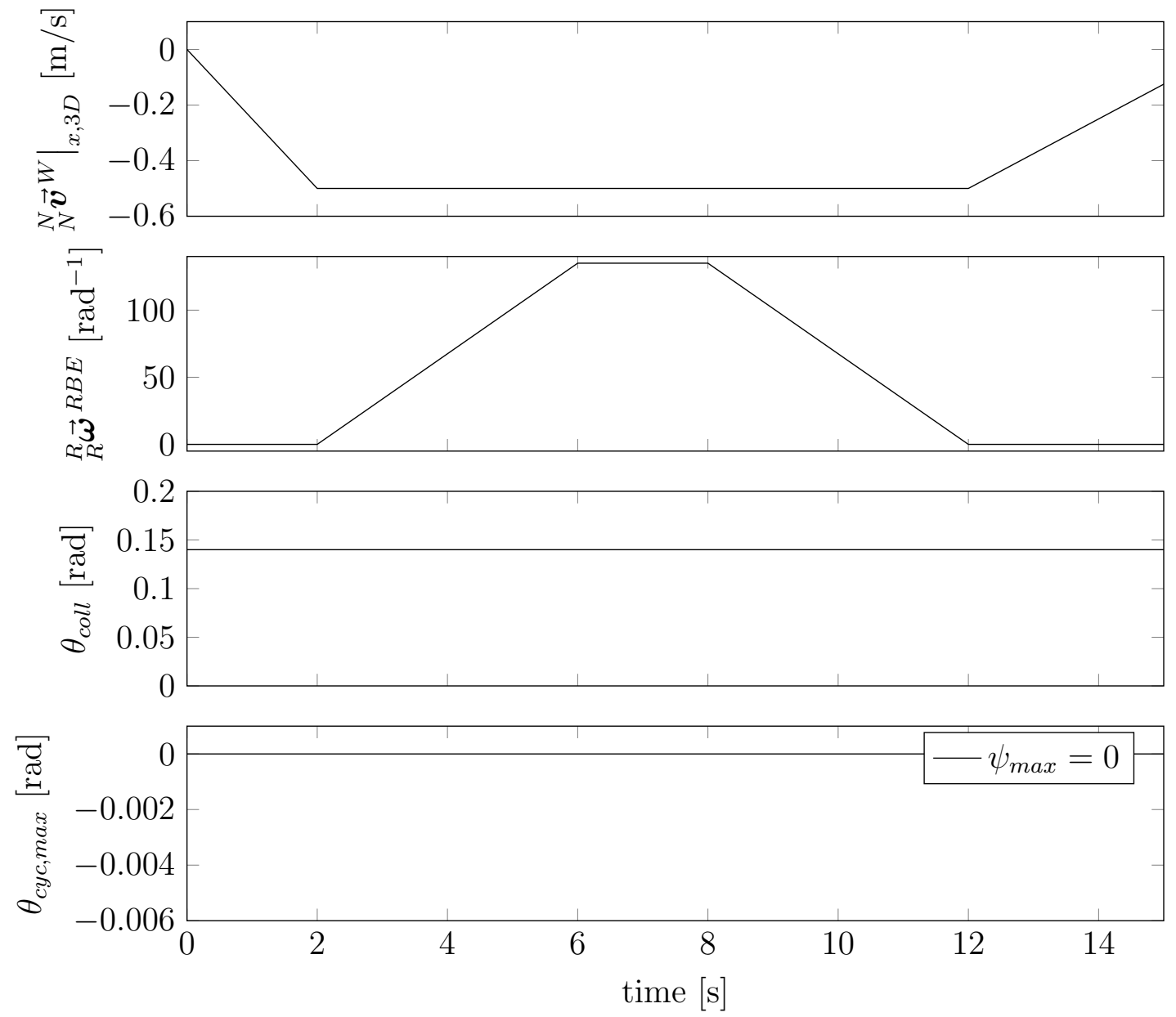

Figure B.2: Case 6.3 Prescribed aerodynamic values. 


\section{B.3 Case 6.4:Rotor Thrust with Head-wind and Cyclic Compensation}
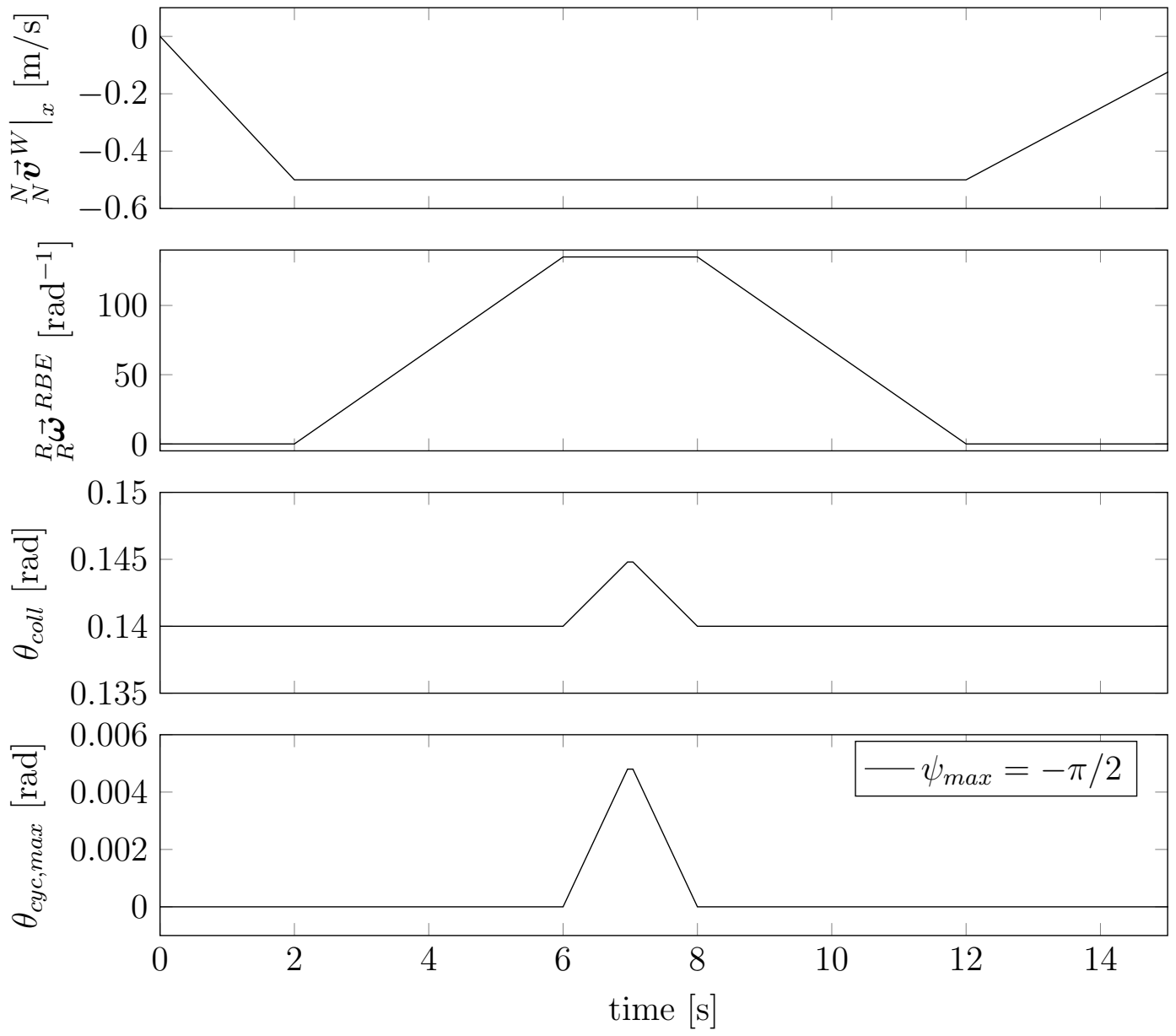

Figure B.3: Case 6.4 Prescribed aerodynamic values. 


\section{Appendix C}

\section{Case 6 Experimental Blade Profiles}

C.1 Case 6.5 S-76 Experimental Blade Profile 


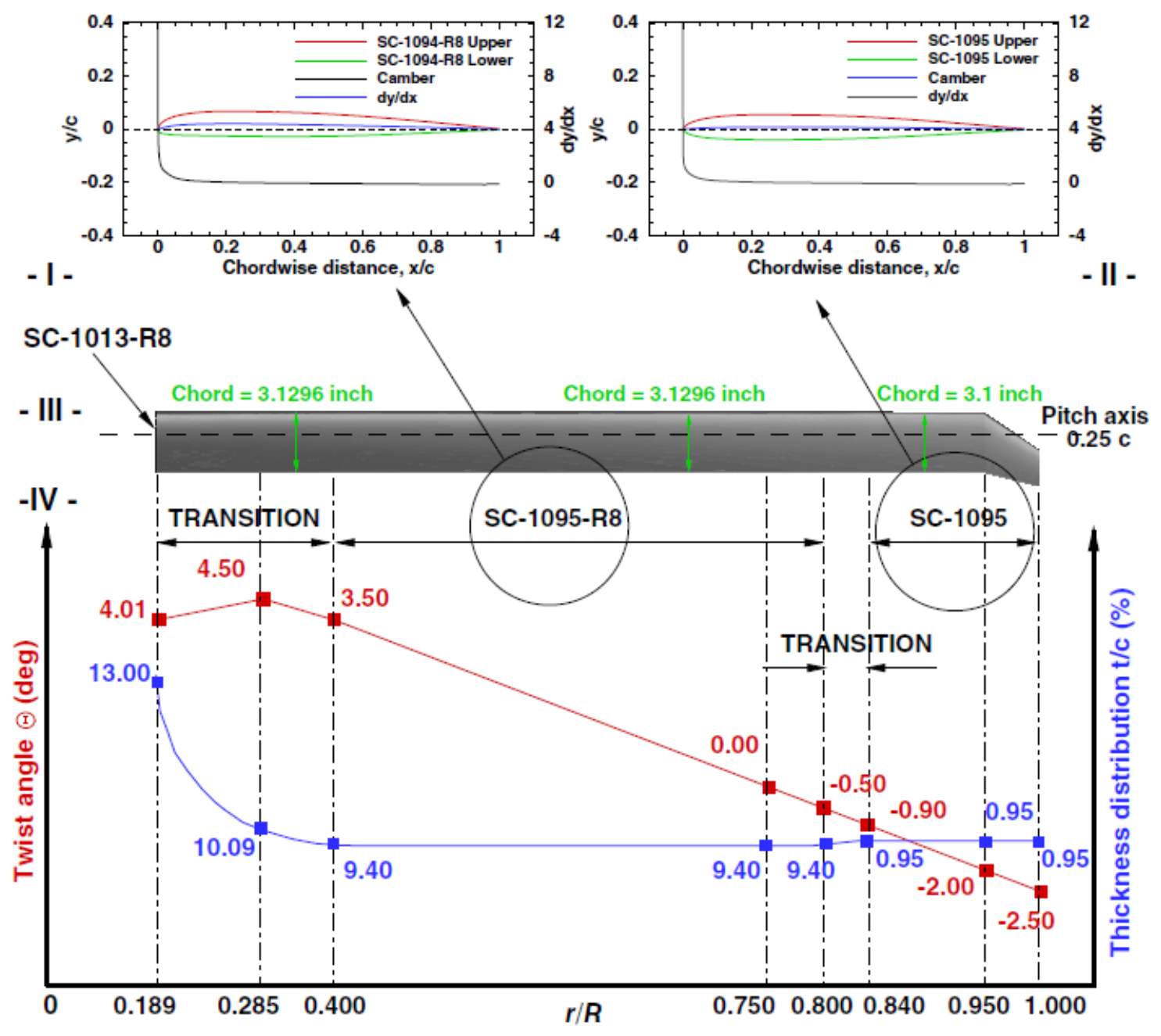

Figure C.1: S-76 model rotor blade profile [7]. 


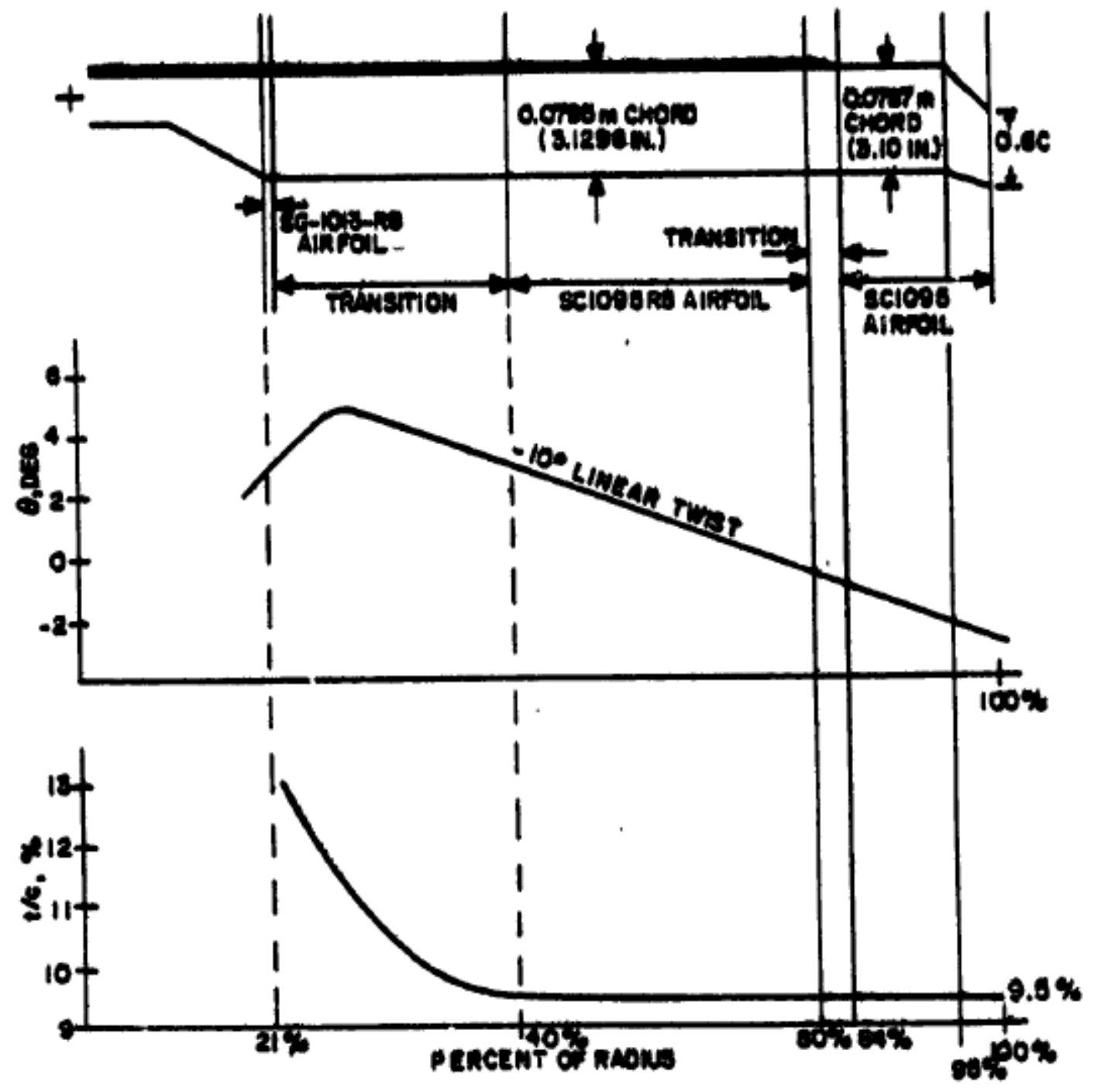

Figure C.2: S-76 model rotor blade profile [8]. 


\section{C.2 Case 6.5 S-76 SRAMSS Blade Profile}
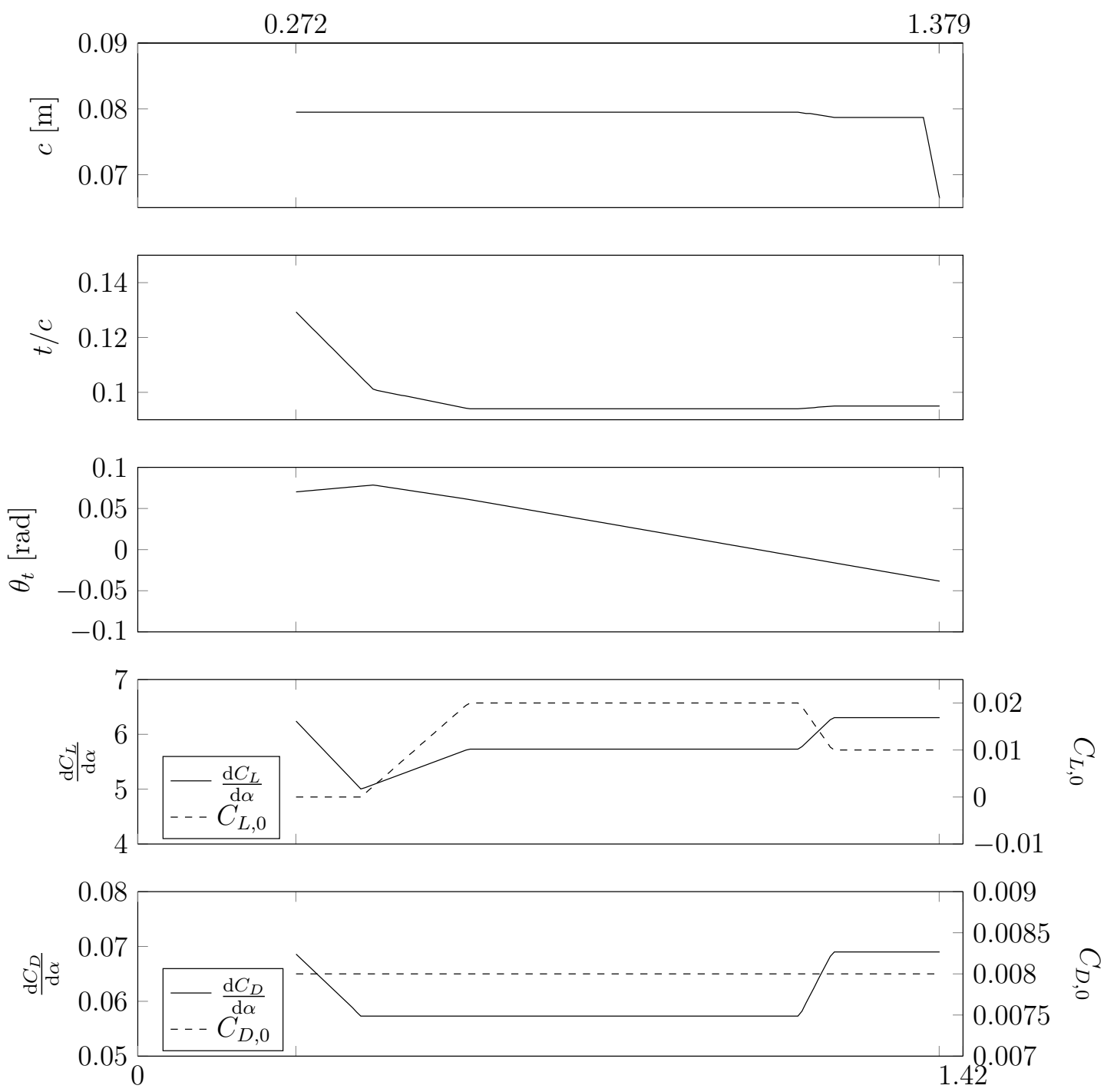

Blade radius $r[\mathrm{~m}]$

Figure C.3: Case 6.5 SRAMSS S-76 model rotor blade profile. 
Table C.1: SRAMS S-76 model BEM parameters

\begin{tabular}{lcc}
\hline Parameter & Variable & Value \\
\hline Number of Rotor Blades & $n_{b}$ & 4 \\
Number of Blade elements & $N_{e}$ & 160 \\
Number of Rotor Disc Sectors & $N_{\psi}$ & 20 \\
Rotor Blade Radius & $R_{b}$ & $1.42 \mathrm{~m}$ \\
Inner Effective Rotor Disc Radius & $R_{i}$ & $0.189(0.272 \mathrm{~m})$ \\
Outer Effective Rotor Disc Radius & $R_{o}$ & $0.972(1.377 \mathrm{~m})$ \\
Solidity ratio & $\sigma$ & 0.0704 \\
Blade Chord Length & $c$ & Fig.C.3 \\
Blade Thickness Ratio & $t / c$ & \\
Blade Twist & $\theta_{t}$ & \\
Uncorrected Lift Coefficient Slope & $\frac{\mathrm{d} C_{L}}{\mathrm{~d} \alpha}$ & \\
Uncorrected Lift Coefficient Offset & $C_{L, 0}$ & \\
Uncorrected Drag Coefficient Slope & $\frac{\mathrm{d} C_{D}}{\mathrm{~d} \alpha}$ & \\
Uncorrected Drag Coefficient Offset & $C_{D, 0}$ & \\
\hline
\end{tabular}




\title{
Appendix D
}

\section{Case 8 Prescribed Parameters}

\author{
D.1 Case 8: Prescribed Aerodynamics
}



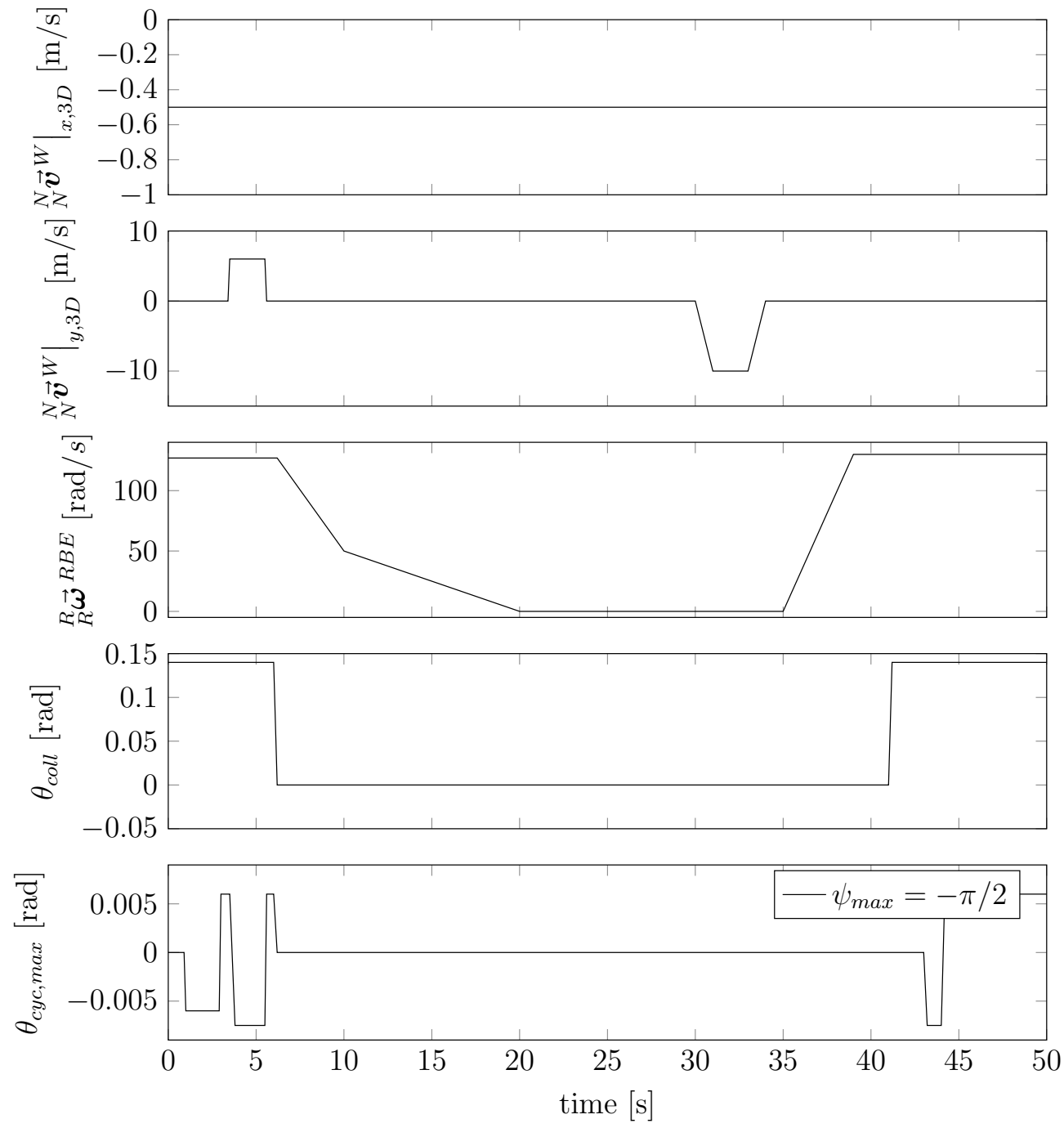

Figure D.1: Case 8 Prescribed aerodynamic values.

\section{D.2 Case 8: Prescribed Motions}



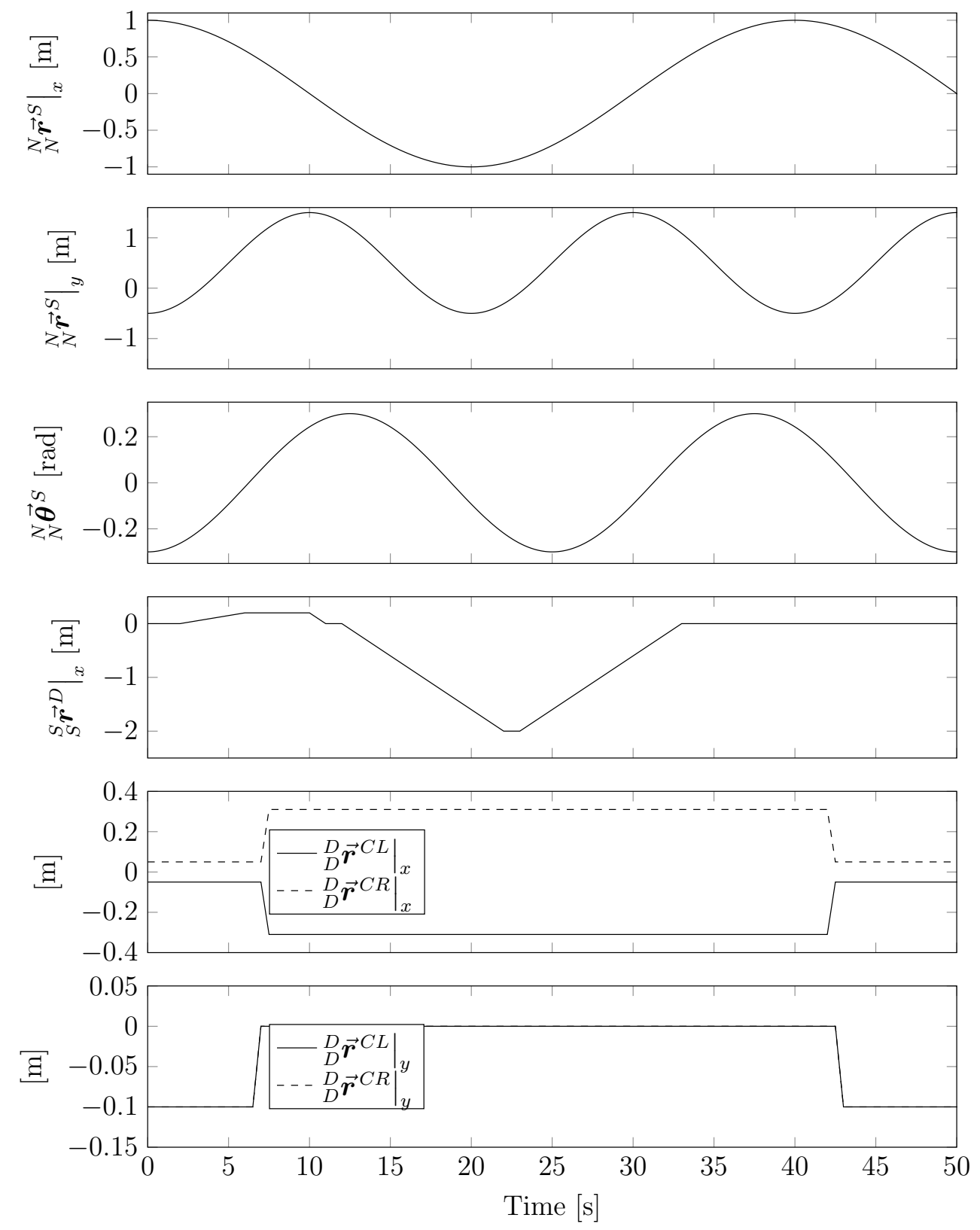

Figure D.2: Case 8 Prescribed polygon motions. 


\section{Appendix E}

\section{Simulation Statistics}

\section{E.1 Case 8: 18/20/160}

\begin{tabular}{llcc} 
step \# & \multicolumn{1}{c}{$\mathrm{t}$} & delt $t / C P U$ & 2441 \\
2400 & 6 & Inf & 2442 \\
2401 & 6.0025 & Inf & 2443 \\
2402 & 6.005 & 0.0533 & 2444 \\
2403 & 6.0075 & Inf & 2445 \\
2404 & 6.01 & 0.04 & 2446 \\
2405 & 6.0125 & Inf & 2447 \\
2406 & 6.015 & Inf & 2448 \\
2407 & 6.0175 & Inf & 2449 \\
2408 & 6.02 & 0.16 & 2450 \\
2409 & 6.0225 & Inf & 2451 \\
2410 & 6.025 & Inf & 2452 \\
2411 & 6.0275 & Inf & 2453 \\
2412 & 6.03 & Inf & 2454 \\
2413 & 6.0325 & Inf & 2455 \\
2414 & 6.035 & Inf & 2456 \\
2415 & 6.0375 & Inf & 2457 \\
2416 & 6.04 & Inf & 2458 \\
2417 & 6.0425 & Inf & 2459 \\
2418 & 6.045 & 0.16 & 2460 \\
2419 & 6.0475 & Inf & 2461 \\
2420 & 6.05 & Inf & 2462 \\
2421 & 6.0525 & Inf & 2463 \\
2422 & 6.055 & Inf & 2464 \\
2423 & 6.0575 & Inf & 2465 \\
2424 & 6.06 & 0.0533 & 2466 \\
2425 & 6.0625 & Inf & 2467 \\
2426 & 6.065 & Inf & 2468 \\
2427 & 6.0675 & Inf & 2469 \\
2428 & 6.07 & Inf & 2470 \\
2429 & 6.0725 & 0.16 & 2471 \\
2430 & 6.075 & Inf & 2472 \\
2431 & 6.0775 & Inf & 2473 \\
2432 & 6.08 & Inf & 2474 \\
2433 & 6.0825 & Inf & 2475 \\
2434 & 6.085 & Inf & 2476 \\
2435 & 6.0875 & Inf & 2477 \\
2436 & 6.09 & Inf & 2478 \\
2437 & 6.0925 & Inf & 2479 \\
2438 & 6.095 & Inf & 2480 \\
2439 & 6.0975 & Inf & 2481 \\
2440 & 6.1 & Inf & 2482
\end{tabular}

$\begin{array}{lcc}6.1025 & \text { Inf } & 2483 \\ 6.105 & \text { Inf } & 2484 \\ 6.1075 & \text { Inf } & 2485 \\ 6.11 & \text { 0.0533 } & 2486 \\ 6.1125 & \text { Inf } & 2487 \\ 6.115 & \text { Inf } & 2488 \\ 6.1175 & \text { Inf } & 2489 \\ 6.12 & \text { Inf } & 2490 \\ 6.1225 & \text { Inf } & 2491 \\ 6.125 & \text { Inf } & 2492 \\ 6.1275 & \text { Inf } & 2493 \\ 6.13 & \text { Inf } & 2494 \\ 6.1325 & \text { Inf } & 2495 \\ 6.135 & \text { Inf } & 2496 \\ 6.1375 & \text { Inf } & 2497 \\ 6.14 & \text { Inf } & 2498 \\ 6.1425 & \text { Inf } & 2499 \\ 6.145 & \text { Inf } & 2500 \\ 6.1475 & \text { Inf } & 2501 \\ 6.15 & \text { O.16 } & 2502 \\ 6.1525 & \text { Inf } & 2503 \\ 6.155 & \text { Inf } & 2504 \\ 6.1575 & \text { Inf } & 2505 \\ 6.16 & \text { 0.0533 } & 2506 \\ 6.1625 & \text { Inf } & 2507 \\ 6.165 & \text { Inf } & 2508 \\ 6.1675 & \text { Inf } & 2509 \\ 6.17 & \text { Inf } & 2510 \\ 6.1725 & \text { Inf } & 2511 \\ 6.175 & \text { Inf } & 2512 \\ 6.1775 & \text { Inf } & 2513 \\ 6.18 & \text { Inf } & 2514 \\ 6.1825 & \text { Inf } & 2515 \\ 6.185 & \text { Inf } & 2516 \\ 6.1875 & \text { Inf } & 2517 \\ 6.19 & \text { 0.16 } & 2518 \\ 6.1925 & \text { Inf } & 2519 \\ 6.195 & \text { Inf } & 2520 \\ 6.1975 & \text { Inf } & 2521 \\ 6.2 & \text { Inf } & 2522 \\ 6.2025 & \text { Inf } & 2523 \\ 6.205 & \text { Inf } & 2524 \\ & & \\ 6.19\end{array}$

$\begin{array}{lcc}6.2075 & \text { Inf } & 2525 \\ 6.21 & 0.0533 & 2526 \\ 6.2125 & 0.16 & 2527 \\ 6.215 & \text { Inf } & 2528 \\ 6.2175 & \text { Inf } & 2529 \\ 6.22 & \text { Inf } & 2530 \\ 6.2225 & \text { Inf } & 2531 \\ 6.225 & \text { Inf } & 2532 \\ 6.2275 & \text { Inf } & 2533 \\ 6.23 & \text { Inf } & 2534 \\ 6.2325 & \text { Inf } & 2535 \\ 6.235 & \text { Inf } & 2536 \\ 6.2375 & \text { Inf } & 2537 \\ 6.24 & \text { Inf } & 2538 \\ 6.2425 & \text { Inf } & 2539 \\ 6.245 & \text { Inf } & 2540 \\ 6.2475 & \text { Inf } & 2541 \\ 6.25 & \text { Inf } & 2542 \\ 6.2525 & \text { O.0533 } & 2543 \\ 6.255 & \text { Inf } & 2544 \\ 6.2575 & \text { Inf } & 2545 \\ 6.26 & \text { Inf } & 2546 \\ 6.2625 & \text { 0.032 } & 2547 \\ 6.265 & \text { Inf } & 2548 \\ 6.2675 & \text { Inf } & 2549 \\ 6.27 & \text { Inf } & 2550 \\ 6.2725 & \text { Inf } & 2551 \\ 6.275 & \text { Inf } & 2552 \\ 6.2775 & \text { Inf } & 2553 \\ 6.28 & \text { Inf } & 2554 \\ 6.2825 & \text { Inf } & 2555 \\ 6.285 & \text { Inf } & 2556 \\ 6.2875 & \text { Inf } & 2557 \\ 6.29 & \text { Inf } & 2558 \\ 6.2925 & \text { Inf } & 2559 \\ 6.295 & \text { Inf } & 2560 \\ 6.2975 & \text { Inf } & 2561 \\ 6.3 & 0.16 & 2562 \\ 6.3025 & \text { Inf } & 2563 \\ 6.305 & \text { Inf } & 2564 \\ 6.3075 & \text { Inf } & 2565 \\ 6.31 & \text { Inf } & 2566 \\ & & \end{array}$

$\begin{array}{lc}6.3125 & 0.0533 \\ 6.315 & \text { Inf } \\ 6.3175 & \text { Inf } \\ 6.32 & \text { Inf } \\ 6.3225 & \text { Inf } \\ 6.325 & \text { Inf } \\ 6.3275 & \text { Inf } \\ 6.33 & 0.16 \\ 6.3325 & \text { Inf } \\ 6.335 & \text { Inf } \\ 6.3375 & \text { Inf } \\ 6.34 & \text { Inf } \\ 6.3425 & \text { Inf } \\ 6.345 & \text { Inf } \\ 6.3475 & \text { Inf } \\ 6.35 & \text { Inf } \\ 6.3525 & \text { Inf } \\ 6.355 & \text { Inf } \\ 6.3575 & \text { Inf } \\ 6.36 & \text { Inf } \\ 6.3625 & \text { O. } 04 \\ 6.365 & \text { Inf } \\ 6.3675 & \text { Inf } \\ 6.37 & \text { Inf } \\ 6.3725 & \text { Inf } \\ 6.375 & \text { Inf } \\ 6.3775 & \text { Inf } \\ 6.38 & \text { Inf } \\ 6.3825 & 0.16 \\ 6.385 & \text { Inf } \\ 6.3875 & \text { Inf } \\ 6.39 & \text { Inf } \\ 6.3925 & \text { Inf } \\ 6.395 & \text { Inf } \\ 6.3975 & \text { Inf } \\ 6.4 & \text { Inf } \\ 6.4025 & \text { Inf } \\ 6.405 & \text { Inf } \\ 6.4075 & \text { Inf } \\ 6.41 & \text { Inf } \\ 6.4125 & 0.032 \\ 6.415 & \text { Inf }\end{array}$




\begin{tabular}{|c|c|c|c|c|c|c|c|c|c|c|c|}
\hline 2567 & 6.4175 & Inf & 2638 & 6.595 & Inf & 2709 & 6.7706 & $\operatorname{Inf}$ & 2780 & 6.9446 & Inf \\
\hline 2568 & 6.42 & Inf & 2639 & 6.5975 & $\operatorname{Inf}$ & 2710 & 6.7731 & 0.0004 & 2781 & 6.9471 & Inf \\
\hline 2569 & 6.4225 & Inf & 2640 & 6.6 & Inf & 2711 & 6.7733 & 0 & 2782 & 6.9496 & Inf \\
\hline 2570 & 6.425 & Inf & 2641 & 6.6025 & $\operatorname{Inf}$ & 2712 & 6.7758 & 0.0001 & 2783 & 6.9521 & Inf \\
\hline 2571 & 6.4275 & $\operatorname{Inf}$ & 2642 & 6.605 & Inf & 2713 & 6.7783 & 0.0001 & 2784 & 6.9546 & Inf \\
\hline 2572 & 6.43 & Inf & 2643 & 6.6075 & Inf & 2714 & 6.7808 & 0.0001 & 2785 & 6.955 & 0.0003 \\
\hline 2573 & 6.4325 & Inf & 2644 & 6.61 & Inf & 2715 & 6.7833 & 0.0001 & 2786 & 6.9575 & 0.0002 \\
\hline 2574 & 6.435 & Inf & 2645 & 6.6125 & 0.04 & 2716 & 6.7858 & 0.0001 & 2787 & 6.96 & Inf \\
\hline 2575 & 6.4375 & $\operatorname{Inf}$ & 2646 & 6.615 & Inf & 2717 & 6.7883 & 0.0001 & 2788 & 6.9625 & Inf \\
\hline 2576 & 6.44 & Inf & 2647 & 6.6175 & $\operatorname{Inf}$ & 2718 & 6.7908 & 0.0001 & 2789 & 6.965 & 0.0533 \\
\hline 2577 & 6.4425 & Inf & 2648 & 6.62 & Inf & 2719 & 6.7933 & 0.0001 & 2790 & 6.9675 & Inf \\
\hline 2578 & 6.445 & Inf & 2649 & 6.6225 & Inf & 2720 & 6.7958 & 0.0013 & 2791 & 6.97 & 0.16 \\
\hline 2579 & 6.4475 & $\operatorname{Inf}$ & 2650 & 6.625 & Inf & 2721 & 6.7983 & 0.0533 & 2792 & 6.9725 & Inf \\
\hline 2580 & 6.45 & Inf & 2651 & 6.6275 & Inf & 2722 & 6.8008 & Inf & 2793 & 6.975 & Inf \\
\hline 2581 & 6.4525 & Inf & 2652 & 6.63 & Inf & 2723 & 6.8033 & Inf & 2794 & 6.9775 & Inf \\
\hline 2582 & 6.455 & Inf & 2653 & 6.6325 & Inf & 2724 & 6.8058 & Inf & 2795 & 6.98 & Inf \\
\hline 2583 & 6.4575 & $\operatorname{Inf}$ & 2654 & 6.635 & Inf & 2725 & 6.8083 & Inf & 2796 & 6.9825 & Inf \\
\hline 2584 & 6.46 & Inf & 2655 & 6.6375 & Inf & 2726 & 6.8108 & Inf & 2797 & 6.985 & Inf \\
\hline 2585 & 6.4625 & 0.04 & 2656 & 6.64 & Inf & 2727 & 6.8133 & 0.04 & 2798 & 6.9875 & 0.0094 \\
\hline 2586 & 6.465 & 0.16 & 2657 & 6.6425 & Inf & 2728 & 6.8146 & 0.0002 & 2799 & 6.9876 & 0.0001 \\
\hline 2587 & 6.4675 & $\operatorname{Inf}$ & 2658 & 6.645 & 0.16 & 2729 & 6.8171 & 0.0001 & 2800 & 6.9901 & 0.0002 \\
\hline 2588 & 6.47 & Inf & 2659 & 6.6475 & $\operatorname{Inf}$ & 2730 & 6.8196 & 0.0001 & 2801 & 6.9926 & 0.04 \\
\hline 2589 & 6.4725 & Inf & 2660 & 6.65 & Inf & 2731 & 6.8221 & 0.0001 & 2802 & 6.9951 & $\operatorname{Inf}$ \\
\hline 2590 & 6.475 & Inf & 2661 & 6.6525 & Inf & 2732 & 6.8246 & 0.0001 & 2803 & 6.9976 & Inf \\
\hline 2591 & 6.4775 & Inf & 2662 & 6.655 & 0.001 & 2733 & 6.8271 & 0.0001 & 2804 & 7.0001 & 0.0013 \\
\hline 2592 & 6.48 & Inf & 2663 & 6.6575 & 0.0001 & 2734 & 6.8296 & 0.0001 & 2805 & 7.0026 & 0.0073 \\
\hline 2593 & 6.4825 & Inf & 2664 & 6.66 & 0.02 & 2735 & 6.8321 & 0.0001 & 2806 & 7.0051 & Inf \\
\hline 2594 & 6.485 & Inf & 2665 & 6.6625 & 0.0533 & 2736 & 6.8346 & 0.0001 & 2807 & 7.0076 & Inf \\
\hline 2595 & 6.4875 & $\operatorname{Inf}$ & 2666 & 6.665 & Inf & 2737 & 6.8371 & 0.0001 & 2808 & 7.0101 & Inf \\
\hline 2596 & 6.49 & Inf & 2667 & 6.6675 & Inf & 2738 & 6.8396 & 0.0001 & 2809 & 7.0126 & Inf \\
\hline 2597 & 6.4925 & 0.16 & 2668 & 6.67 & Inf & 2739 & 6.8421 & 0.0001 & 2810 & 7.0151 & Inf \\
\hline 2598 & 6.495 & Inf & 2669 & 6.6725 & Inf & 2740 & 6.8446 & 0.0001 & 2811 & 7.0176 & Inf \\
\hline 2599 & 6.4975 & $\operatorname{Inf}$ & 2670 & 6.675 & 0.0533 & 2741 & 6.8471 & 0.0057 & 2812 & 7.0201 & Inf \\
\hline 2600 & 6.5 & Inf & 2671 & 6.6775 & Inf & 2742 & 6.8496 & 0.0533 & 2813 & 7.0226 & Inf \\
\hline 2601 & 6.5025 & $\operatorname{Inf}$ & 2672 & 6.68 & 0.0229 & 2743 & 6.8521 & Inf & 2814 & 7.0251 & Inf \\
\hline 2602 & 6.505 & Inf & 2673 & 6.6806 & 0.0003 & 2744 & 6.8546 & Inf & 2815 & 7.0276 & Inf \\
\hline 2603 & 6.5075 & $\operatorname{Inf}$ & 2674 & 6.6831 & 0.0002 & 2745 & 6.8571 & Inf & 2816 & 7.0301 & Inf \\
\hline 2604 & 6.51 & Inf & 2675 & 6.6856 & 0.04 & 2746 & 6.8596 & Inf & 2817 & 7.0326 & 0.16 \\
\hline 2605 & 6.5125 & 0.0533 & 2676 & 6.6881 & Inf & 2747 & 6.8621 & 0.16 & 2818 & 7.0351 & Inf \\
\hline 2606 & 6.515 & Inf & 2677 & 6.6906 & 0.04 & 2748 & 6.8646 & Inf & 2819 & 7.0376 & Inf \\
\hline 2607 & 6.5175 & Inf & 2678 & 6.6931 & Inf & 2749 & 6.8671 & Inf & 2820 & 7.0401 & Inf \\
\hline 2608 & 6.52 & Inf & 2679 & 6.6956 & Inf & 2750 & 6.8696 & Inf & 2821 & 7.0426 & Inf \\
\hline 2609 & 6.5225 & $\operatorname{Inf}$ & 2680 & 6.6981 & 0.16 & 2751 & 6.8721 & Inf & 2822 & 7.0451 & Inf \\
\hline 2610 & 6.525 & Inf & 2681 & 6.7006 & 0.0009 & 2752 & 6.8746 & Inf & 2823 & 7.0476 & Inf \\
\hline 2611 & 6.5275 & Inf & 2682 & 6.7031 & 0.0229 & 2753 & 6.8771 & Inf & 2824 & 7.0501 & Inf \\
\hline 2612 & 6.53 & Inf & 2683 & 6.7056 & 0.0533 & 2754 & 6.8796 & Inf & 2825 & 7.0526 & 0.0123 \\
\hline 2613 & 6.5325 & Inf & 2684 & 6.7081 & Inf & 2755 & 6.8821 & Inf & 2826 & 7.0528 & 0.0001 \\
\hline 2614 & 6.535 & Inf & 2685 & 6.7106 & Inf & 2756 & 6.8846 & Inf & 2827 & 7.0539 & 0.0001 \\
\hline 2615 & 6.5375 & $\operatorname{Inf}$ & 2686 & 6.7131 & Inf & 2757 & 6.8871 & Inf & 2828 & 7.0564 & 0.0003 \\
\hline 2616 & 6.54 & Inf & 2687 & 6.7156 & Inf & 2758 & 6.8896 & $\operatorname{Inf}$ & 2829 & 7.0589 & 0.0048 \\
\hline 2617 & 6.5425 & Inf & 2688 & 6.7181 & Inf & 2759 & 6.8921 & Inf & 2830 & 7.0614 & 0.0533 \\
\hline 2618 & 6.545 & Inf & 2689 & 6.7206 & $\operatorname{Inf}$ & 2760 & 6.8946 & Inf & 2831 & 7.0639 & Inf \\
\hline 2619 & 6.5475 & Inf & 2690 & 6.7231 & Inf & 2761 & 6.8971 & Inf & 2832 & 7.0664 & Inf \\
\hline 2620 & 6.55 & Inf & 2691 & 6.7256 & 0.0015 & 2762 & 6.8996 & 0.04 & 2833 & 7.0689 & 0.16 \\
\hline 2621 & 6.5525 & Inf & 2692 & 6.7281 & Inf & 2763 & 6.9021 & Inf & 2834 & 7.0714 & Inf \\
\hline 2622 & 6.555 & Inf & 2693 & 6.7306 & $\operatorname{Inf}$ & 2764 & 6.9046 & Inf & 2835 & 7.0739 & Inf \\
\hline 2623 & 6.5575 & Inf & 2694 & 6.7331 & Inf & 2765 & 6.9071 & Inf & 2836 & 7.0764 & Inf \\
\hline 2624 & 6.56 & Inf & 2695 & 6.7356 & Inf & 2766 & 6.9096 & Inf & 2837 & 7.0789 & Inf \\
\hline 2625 & 6.5625 & 0.0533 & 2696 & 6.7381 & Inf & 2767 & 6.9121 & 0.0533 & 2838 & 7.0814 & Inf \\
\hline 2626 & 6.565 & Inf & 2697 & 6.7406 & 0.0229 & 2768 & 6.9146 & Inf & 2839 & 7.0839 & Inf \\
\hline 2627 & 6.5675 & $\operatorname{Inf}$ & 2698 & 6.7431 & 0.04 & 2769 & 6.9171 & Inf & 2840 & 7.0864 & 0.04 \\
\hline 2628 & 6.57 & Inf & 2699 & 6.7456 & Inf & 2770 & 6.9196 & Inf & 2841 & 7.0889 & Inf \\
\hline 2629 & 6.5725 & Inf & 2700 & 6.7481 & Inf & 2771 & 6.9221 & Inf & 2842 & 7.0914 & Inf \\
\hline 2630 & 6.575 & Inf & 2701 & 6.7506 & 0.0533 & 2772 & 6.9246 & Inf & 2843 & 7.0939 & Inf \\
\hline 2631 & 6.5775 & $\operatorname{Inf}$ & 2702 & 6.7531 & Inf & 2773 & 6.9271 & 0.16 & 2844 & 7.0964 & Inf \\
\hline 2632 & 6.58 & Inf & 2703 & 6.7556 & Inf & 2774 & 6.9296 & Inf & 2845 & 7.0989 & 0.0229 \\
\hline 2633 & 6.5825 & Inf & 2704 & 6.7581 & Inf & 2775 & 6.9321 & Inf & 2846 & 7.1014 & 0.0008 \\
\hline 2634 & 6.585 & Inf & 2705 & 6.7606 & Inf & 2776 & 6.9346 & Inf & 2847 & 7.1039 & Inf \\
\hline 2635 & 6.5875 & 0.16 & 2706 & 6.7631 & Inf & 2777 & 6.9371 & Inf & 2848 & 7.1064 & Inf \\
\hline 2636 & 6.59 & Inf & 2707 & 6.7656 & Inf & 2778 & 6.9396 & Inf & 2849 & 7.1089 & 0.04 \\
\hline 2637 & 6.5925 & Inf & 2708 & 6.7681 & 0.16 & 2779 & 6.9421 & Inf & 2850 & 7.1114 & Inf \\
\hline
\end{tabular}




\begin{tabular}{|c|c|c|c|c|c|c|c|c|c|c|c|}
\hline 2851 & 7.1139 & Inf & 2922 & 7.2892 & Inf & 2993 & 7.4667 & Inf & 3064 & 7.6277 & Inf \\
\hline 2852 & 7.1164 & Inf & 2923 & 7.2917 & Inf & 2994 & 7.4692 & Inf & 3065 & 7.6301 & 0.0013 \\
\hline 2853 & 7.1189 & Inf & 2924 & 7.2942 & Inf & 2995 & 7.4717 & Inf & 3066 & 7.6326 & 0.0003 \\
\hline 2854 & 7.12 & 0.0008 & 2925 & 7.2967 & Inf & 2996 & 7.4742 & Inf & 3067 & 7.6351 & 0.0533 \\
\hline 2855 & 7.1225 & 0.0001 & 2926 & 7.2992 & Inf & 2997 & 7.4767 & Inf & 3068 & 7.6376 & 0.04 \\
\hline 2856 & 7.1244 & 0.0015 & 2927 & 7.3017 & 0.0002 & 2998 & 7.4792 & Inf & 3069 & 7.6401 & 0.0267 \\
\hline 2857 & 7.1269 & 0.0003 & 2928 & 7.3042 & 0.0267 & 2999 & 7.4817 & 0.16 & 3070 & 7.6421 & 0.0015 \\
\hline 2858 & 7.1294 & 0.04 & 2929 & 7.3067 & Inf & 3000 & 7.4836 & 0.0009 & 3071 & 7.6446 & 0.0008 \\
\hline 2859 & 7.1317 & 0.0018 & 2930 & 7.3092 & 0.04 & 3001 & 7.4844 & 0.0001 & 3072 & 7.6471 & 0.0533 \\
\hline 2860 & 7.1342 & 0.0007 & 2931 & 7.3117 & Inf & 3002 & 7.4869 & 0.0002 & 3073 & 7.6496 & Inf \\
\hline 2861 & 7.1367 & 0.0533 & 2932 & 7.3142 & Inf & 3003 & 7.4894 & 0.0267 & 3074 & 7.6521 & 0.0533 \\
\hline 2862 & 7.1392 & 0.16 & 2933 & 7.3167 & Inf & 3004 & 7.4919 & 0.16 & 3075 & 7.6546 & Inf \\
\hline 2863 & 7.1417 & 0.005 & 2934 & 7.3192 & Inf & 3005 & 7.4944 & 0.08 & 3076 & 7.6571 & Inf \\
\hline 2864 & 7.1442 & 0.0022 & 2935 & 7.3217 & Inf & 3006 & 7.4969 & Inf & 3077 & 7.6596 & Inf \\
\hline 2865 & 7.1467 & 0.0019 & 2936 & 7.3242 & Inf & 3007 & 7.4994 & 0.0229 & 3078 & 7.6621 & Inf \\
\hline 2866 & 7.1492 & 0.0024 & 2937 & 7.3267 & Inf & 3008 & 7.5019 & 0.0002 & 3079 & 7.6646 & Inf \\
\hline 2867 & 7.1517 & 0.04 & 2938 & 7.3292 & Inf & 3009 & 7.5019 & 0 & 3080 & 7.6671 & 0.16 \\
\hline 2868 & 7.1542 & Inf & 2939 & 7.3317 & Inf & 3010 & 7.5044 & 0.0002 & 3081 & 7.6696 & Inf \\
\hline 2869 & 7.1567 & Inf & 2940 & 7.3342 & Inf & 3011 & 7.5069 & 0.0267 & 3082 & 7.6721 & Inf \\
\hline 2870 & 7.1592 & Inf & 2941 & 7.3367 & Inf & 3012 & 7.5094 & Inf & 3083 & 7.6746 & Inf \\
\hline 2871 & 7.1617 & Inf & 2942 & 7.3392 & Inf & 3013 & 7.5119 & Inf & 3084 & 7.6771 & Inf \\
\hline 2872 & 7.1642 & 0.04 & 2943 & 7.3417 & 0.16 & 3014 & 7.5144 & Inf & 3085 & 7.6796 & Inf \\
\hline 2873 & 7.1667 & 0.0094 & 2944 & 7.3442 & Inf & 3015 & 7.5169 & 0.0533 & 3086 & 7.6821 & Inf \\
\hline 2874 & 7.1692 & 0.016 & 2945 & 7.3467 & Inf & 3016 & 7.5194 & 0.0229 & 3087 & 7.6846 & Inf \\
\hline 2875 & 7.1717 & Inf & 2946 & 7.3492 & Inf & 3017 & 7.5219 & 0.0533 & 3088 & 7.6871 & Inf \\
\hline 2876 & 7.1742 & Inf & 2947 & 7.3517 & Inf & 3018 & 7.5244 & 0.016 & 3089 & 7.6896 & Inf \\
\hline 2877 & 7.1767 & Inf & 2948 & 7.3542 & Inf & 3019 & 7.5266 & 0.001 & 3090 & 7.6921 & Inf \\
\hline 2878 & 7.1792 & Inf & 2949 & 7.3567 & Inf & 3020 & 7.5272 & 0 & 3091 & 7.6946 & Inf \\
\hline 2879 & 7.1817 & Inf & 2950 & 7.3592 & 0.0533 & 3021 & 7.5297 & 0.0002 & 3092 & 7.6971 & Inf \\
\hline 2880 & 7.1842 & Inf & 2951 & 7.3617 & Inf & 3022 & 7.5322 & 0.0038 & 3093 & 7.6996 & 0.016 \\
\hline 2881 & 7.1867 & Inf & 2952 & 7.3642 & Inf & 3023 & 7.5347 & 0.04 & 3094 & 7.7021 & 0.0008 \\
\hline 2882 & 7.1892 & Inf & 2953 & 7.3667 & Inf & 3024 & 7.5372 & Inf & 3095 & 7.7046 & 0.0533 \\
\hline 2883 & 7.1917 & Inf & 2954 & 7.3692 & Inf & 3025 & 7.5397 & Inf & 3096 & 7.7071 & Inf \\
\hline 2884 & 7.1942 & Inf & 2955 & 7.3717 & Inf & 3026 & 7.5422 & Inf & 3097 & 7.7096 & Inf \\
\hline 2885 & 7.1967 & 0.08 & 2956 & 7.3742 & Inf & 3027 & 7.5447 & 0.04 & 3098 & 7.7121 & Inf \\
\hline 2886 & 7.1992 & Inf & 2957 & 7.3767 & Inf & 3028 & 7.5472 & Inf & 3099 & 7.7146 & Inf \\
\hline 2887 & 7.2017 & 0.0004 & 2958 & 7.3792 & Inf & 3029 & 7.5497 & Inf & 3100 & 7.7171 & 0.16 \\
\hline 2888 & 7.2042 & Inf & 2959 & 7.3817 & Inf & 3030 & 7.5522 & $\operatorname{Inf}$ & 3101 & 7.7196 & Inf \\
\hline 2889 & 7.2067 & 0.04 & 2960 & 7.3842 & Inf & 3031 & 7.5547 & Inf & 3102 & 7.7221 & Inf \\
\hline 2890 & 7.2092 & Inf & 2961 & 7.3867 & Inf & 3032 & 7.5572 & 0.0012 & 3103 & 7.7246 & Inf \\
\hline 2891 & 7.2117 & 0.0533 & 2962 & 7.3892 & Inf & 3033 & 7.5597 & 0.0002 & 3104 & 7.7271 & Inf \\
\hline 2892 & 7.2142 & Inf & 2963 & 7.3917 & Inf & 3034 & 7.5616 & 0.0005 & 3105 & 7.7296 & Inf \\
\hline 2893 & 7.2167 & Inf & 2964 & 7.3942 & Inf & 3035 & 7.5641 & 0.0003 & 3106 & 7.7321 & Inf \\
\hline 2894 & 7.2192 & Inf & 2965 & 7.3967 & Inf & 3036 & 7.5666 & 0.0031 & 3107 & 7.7346 & Inf \\
\hline 2895 & 7.2217 & Inf & 2966 & 7.3992 & 0.016 & 3037 & 7.5691 & Inf & 3108 & 7.7371 & Inf \\
\hline 2896 & 7.2242 & Inf & 2967 & 7.4017 & 0.0006 & 3038 & 7.5716 & 0.04 & 3109 & 7.7396 & Inf \\
\hline 2897 & 7.2267 & Inf & 2968 & 7.4042 & 0.032 & 3039 & 7.5741 & Inf & 3110 & 7.7421 & Inf \\
\hline 2898 & 7.2292 & 0.16 & 2969 & 7.4067 & 0.16 & 3040 & 7.5766 & Inf & 3111 & 7.7446 & Inf \\
\hline 2899 & 7.2317 & Inf & 2970 & 7.4092 & 0.0533 & 3041 & 7.5791 & Inf & 3112 & 7.7471 & Inf \\
\hline 2900 & 7.2342 & Inf & 2971 & 7.4117 & Inf & 3042 & 7.5816 & Inf & 3113 & 7.7496 & Inf \\
\hline 2901 & 7.2367 & Inf & 2972 & 7.4142 & Inf & 3043 & 7.5841 & 0.032 & 3114 & 7.7521 & 0.0533 \\
\hline 2902 & 7.2392 & Inf & 2973 & 7.4167 & Inf & 3044 & 7.5849 & 0.0006 & 3115 & 7.7546 & Inf \\
\hline 2903 & 7.2417 & Inf & 2974 & 7.4192 & Inf & 3045 & 7.5874 & 0.0004 & 3116 & 7.7571 & 0.16 \\
\hline 2904 & 7.2442 & Inf & 2975 & 7.4217 & Inf & 3046 & 7.5899 & 0.016 & 3117 & 7.7596 & Inf \\
\hline 2905 & 7.2467 & Inf & 2976 & 7.4242 & Inf & 3047 & 7.5907 & 0.0004 & 3118 & 7.7621 & Inf \\
\hline 2906 & 7.2492 & Inf & 2977 & 7.4267 & Inf & 3048 & 7.5932 & 0.0003 & 3119 & 7.7646 & Inf \\
\hline 2907 & 7.2517 & Inf & 2978 & 7.4292 & Inf & 3049 & 7.5957 & Inf & 3120 & 7.7671 & Inf \\
\hline 2908 & 7.2542 & 0.04 & 2979 & 7.4317 & Inf & 3050 & 7.5982 & 0.0533 & 3121 & 7.7696 & Inf \\
\hline 2909 & 7.2567 & Inf & 2980 & 7.4342 & Inf & 3051 & 7.6007 & 0.0007 & 3122 & 7.7721 & Inf \\
\hline 2910 & 7.2592 & Inf & 2981 & 7.4367 & Inf & 3052 & 7.6031 & 0.0013 & 3123 & 7.7746 & Inf \\
\hline 2911 & 7.2617 & 0.0533 & 2982 & 7.4392 & Inf & 3053 & 7.6056 & 0.0002 & 3124 & 7.7771 & Inf \\
\hline 2912 & 7.2642 & $\operatorname{Inf}$ & 2983 & 7.4417 & Inf & 3054 & 7.6081 & 0.0009 & 3125 & 7.7796 & Inf \\
\hline 2913 & 7.2667 & Inf & 2984 & 7.4442 & Inf & 3055 & 7.6106 & 0.016 & 3126 & 7.7821 & Inf \\
\hline 2914 & 7.2692 & Inf & 2985 & 7.4467 & Inf & 3056 & 7.6109 & 0.0003 & 3127 & 7.7846 & Inf \\
\hline 2915 & 7.2717 & Inf & 2986 & 7.4492 & Inf & 3057 & 7.6134 & 0.0008 & 3128 & 7.7871 & Inf \\
\hline 2916 & 7.2742 & Inf & 2987 & 7.4517 & Inf & 3058 & 7.6149 & 0.0009 & 3129 & 7.7896 & Inf \\
\hline 2917 & 7.2767 & 0.16 & 2988 & 7.4542 & Inf & 3059 & 7.6152 & 0.0001 & 3130 & 7.7921 & Inf \\
\hline 2918 & 7.2792 & Inf & 2989 & 7.4567 & Inf & 3060 & 7.6177 & 0.0006 & 3131 & 7.7946 & Inf \\
\hline 2919 & 7.2817 & Inf & 2990 & 7.4592 & 0.0533 & 3061 & 7.6202 & 0.0533 & 3132 & 7.7971 & Inf \\
\hline 2920 & 7.2842 & $\operatorname{Inf}$ & 2991 & 7.4617 & Inf & 3062 & 7.6227 & Inf & 3133 & 7.7996 & 0.0229 \\
\hline 2921 & 7.2867 & Inf & 2992 & 7.4642 & Inf & 3063 & 7.6252 & Inf & 3134 & 7.8021 & 0.0009 \\
\hline
\end{tabular}




\begin{tabular}{|c|c|c|c|c|c|c|c|c|c|c|c|}
\hline 3135 & 7.8046 & 0.0533 & 3206 & 7.9821 & 0.16 & 3277 & 8.1596 & Inf & 3348 & 8.3371 & Inf \\
\hline 3136 & 7.8071 & Inf & 3207 & 7.9846 & Inf & 3278 & 8.1621 & 0.0533 & 3349 & 8.3396 & Inf \\
\hline 3137 & 7.8096 & Inf & 3208 & 7.9871 & Inf & 3279 & 8.1646 & Inf & 3350 & 8.3421 & Inf \\
\hline 3138 & 7.8121 & Inf & 3209 & 7.9896 & Inf & 3280 & 8.1671 & Inf & 3351 & 8.3446 & Inf \\
\hline 3139 & 7.8146 & 0.0533 & 3210 & 7.9921 & Inf & 3281 & 8.1696 & Inf & 3352 & 8.3471 & Inf \\
\hline 3140 & 7.8171 & Inf & 3211 & 7.9946 & Inf & 3282 & 8.1721 & Inf & 3353 & 8.3496 & Inf \\
\hline 3141 & 7.8196 & Inf & 3212 & 7.9971 & Inf & 3283 & 8.1746 & Inf & 3354 & 8.3521 & Inf \\
\hline 3142 & 7.8221 & Inf & 3213 & 7.9996 & 0.0178 & 3284 & 8.1771 & Inf & 3355 & 8.3546 & Inf \\
\hline 3143 & 7.8246 & Inf & 3214 & 8.0021 & 0.0005 & 3285 & 8.1796 & Inf & 3356 & 8.3571 & Inf \\
\hline 3144 & 7.8271 & Inf & 3215 & 8.0046 & 0.0003 & 3286 & 8.1821 & 0.0533 & 3357 & 8.3596 & Inf \\
\hline 3145 & 7.8296 & Inf & 3216 & 8.0071 & 0.0003 & 3287 & 8.1846 & Inf & 3358 & 8.3621 & Inf \\
\hline 3146 & 7.8321 & 0.16 & 3217 & 8.0096 & 0.0001 & 3288 & 8.1871 & Inf & 3359 & 8.3646 & Inf \\
\hline 3147 & 7.8346 & Inf & 3218 & 8.0121 & 0.0001 & 3289 & 8.1896 & Inf & 3360 & 8.3671 & Inf \\
\hline 3148 & 7.8371 & Inf & 3219 & 8.0146 & 0.0001 & 3290 & 8.1921 & Inf & 3361 & 8.3696 & Inf \\
\hline 3149 & 7.8396 & Inf & 3220 & 8.0171 & 0.0001 & 3291 & 8.1946 & Inf & 3362 & 8.3721 & Inf \\
\hline 3150 & 7.8421 & Inf & 3221 & 8.0196 & 0.0001 & 3292 & 8.1971 & 0.0533 & 3363 & 8.3746 & Inf \\
\hline 3151 & 7.8446 & Inf & 3222 & 8.0221 & 0.0001 & 3293 & 8.1996 & Inf & 3364 & 8.3771 & 0.0533 \\
\hline 3152 & 7.8471 & Inf & 3223 & 8.0246 & 0.0001 & 3294 & 8.2021 & 0.0009 & 3365 & 8.3796 & Inf \\
\hline 3153 & 7.8496 & Inf & 3224 & 8.0271 & 0.0002 & 3295 & 8.2046 & 0.0533 & 3366 & 8.3821 & Inf \\
\hline 3154 & 7.8521 & Inf & 3225 & 8.0296 & Inf & 3296 & 8.2071 & Inf & 3367 & 8.3846 & 0.16 \\
\hline 3155 & 7.8546 & Inf & 3226 & 8.0321 & 0.04 & 3297 & 8.2096 & Inf & 3368 & 8.3871 & Inf \\
\hline 3156 & 7.8571 & Inf & 3227 & 8.0346 & Inf & 3298 & 8.2121 & Inf & 3369 & 8.3896 & Inf \\
\hline 3157 & 7.8596 & Inf & 3228 & 8.0371 & 0.0533 & 3299 & 8.2146 & 0.04 & 3370 & 8.3921 & Inf \\
\hline 3158 & 7.8621 & Inf & 3229 & 8.0396 & Inf & 3300 & 8.2171 & Inf & 3371 & 8.3946 & Inf \\
\hline 3159 & 7.8646 & 0.0533 & 3230 & 8.0421 & Inf & 3301 & 8.2196 & Inf & 3372 & 8.3971 & Inf \\
\hline 3160 & 7.8671 & Inf & 3231 & 8.0446 & Inf & 3302 & 8.2221 & Inf & 3373 & 8.3996 & 0.0133 \\
\hline 3161 & 7.8696 & Inf & 3232 & 8.0471 & Inf & 3303 & 8.2246 & Inf & 3374 & 8.4021 & 0.001 \\
\hline 3162 & 7.8721 & 0.16 & 3233 & 8.0496 & Inf & 3304 & 8.2271 & Inf & 3375 & 8.4046 & 0.0533 \\
\hline 3163 & 7.8746 & Inf & 3234 & 8.0521 & 0.16 & 3305 & 8.2296 & Inf & 3376 & 8.4071 & Inf \\
\hline 3164 & 7.8771 & Inf & 3235 & 8.0546 & Inf & 3306 & 8.2321 & Inf & 3377 & 8.4096 & Inf \\
\hline 3165 & 7.8796 & 0.0533 & 3236 & 8.0571 & Inf & 3307 & 8.2346 & Inf & 3378 & 8.4121 & Inf \\
\hline 3166 & 7.8821 & Inf & 3237 & 8.0596 & Inf & 3308 & 8.2371 & Inf & 3379 & 8.4146 & Inf \\
\hline 3167 & 7.8846 & Inf & 3238 & 8.0621 & Inf & 3309 & 8.2396 & Inf & 3380 & 8.4171 & Inf \\
\hline 3168 & 7.8871 & Inf & 3239 & 8.0646 & Inf & 3310 & 8.2421 & Inf & 3381 & 8.4196 & Inf \\
\hline 3169 & 7.8896 & Inf & 3240 & 8.0671 & Inf & 3311 & 8.2446 & Inf & 3382 & 8.4221 & Inf \\
\hline 3170 & 7.8921 & Inf & 3241 & 8.0696 & Inf & 3312 & 8.2471 & Inf & 3383 & 8.4246 & Inf \\
\hline 3171 & 7.8946 & 0.0533 & 3242 & 8.0721 & Inf & 3313 & 8.2496 & Inf & 3384 & 8.4271 & 0.04 \\
\hline 3172 & 7.8971 & Inf & 3243 & 8.0746 & Inf & 3314 & 8.2521 & Inf & 3385 & 8.4296 & Inf \\
\hline 3173 & 7.8996 & 0.0229 & 3244 & 8.0771 & Inf & 3315 & 8.2546 & Inf & 3386 & 8.4321 & Inf \\
\hline 3174 & 7.9021 & 0.0012 & 3245 & 8.0796 & Inf & 3316 & 8.2571 & Inf & 3387 & 8.4346 & Inf \\
\hline 3175 & 7.9046 & Inf & 3246 & 8.0821 & Inf & 3317 & 8.2596 & 0.16 & 3388 & 8.4371 & Inf \\
\hline 3176 & 7.9071 & 0.04 & 3247 & 8.0846 & Inf & 3318 & 8.2621 & Inf & 3389 & 8.4396 & Inf \\
\hline 3177 & 7.9096 & Inf & 3248 & 8.0871 & 0.0533 & 3319 & 8.2646 & 0.0533 & 3390 & 8.4421 & Inf \\
\hline 3178 & 7.9121 & 0.0533 & 3249 & 8.0896 & 0.16 & 3320 & 8.2671 & Inf & 3391 & 8.4446 & Inf \\
\hline 3179 & 7.9146 & Inf & 3250 & 8.0921 & Inf & 3321 & 8.2696 & Inf & 3392 & 8.4471 & Inf \\
\hline 3180 & 7.9171 & Inf & 3251 & 8.0946 & Inf & 3322 & 8.2721 & Inf & 3393 & 8.4496 & Inf \\
\hline 3181 & 7.9196 & Inf & 3252 & 8.0971 & Inf & 3323 & 8.2746 & Inf & 3394 & 8.4521 & Inf \\
\hline 3182 & 7.9221 & Inf & 3253 & 8.0996 & Inf & 3324 & 8.2771 & Inf & 3395 & 8.4546 & Inf \\
\hline 3183 & 7.9246 & Inf & 3254 & 8.1021 & 0.0004 & 3325 & 8.2796 & 0.0533 & 3396 & 8.4571 & 0.16 \\
\hline 3184 & 7.9271 & Inf & 3255 & 8.1046 & 0.0005 & 3326 & 8.2821 & Inf & 3397 & 8.4596 & Inf \\
\hline 3185 & 7.9296 & Inf & 3256 & 8.1071 & 0.08 & 3327 & 8.2846 & Inf & 3398 & 8.4621 & Inf \\
\hline 3186 & 7.9321 & Inf & 3257 & 8.1096 & Inf & 3328 & 8.2871 & Inf & 3399 & 8.4646 & Inf \\
\hline 3187 & 7.9346 & Inf & 3258 & 8.1121 & 0.04 & 3329 & 8.2896 & Inf & 3400 & 8.4671 & Inf \\
\hline 3188 & 7.9371 & $\operatorname{Inf}$ & 3259 & 8.1146 & Inf & 3330 & 8.2921 & Inf & 3401 & 8.4696 & Inf \\
\hline 3189 & 7.9396 & Inf & 3260 & 8.1171 & Inf & 3331 & 8.2946 & 0.04 & 3402 & 8.4721 & Inf \\
\hline 3190 & 7.9421 & Inf & 3261 & 8.1196 & Inf & 3332 & 8.2971 & Inf & 3403 & 8.4746 & Inf \\
\hline 3191 & 7.9446 & Inf & 3262 & 8.1221 & Inf & 3333 & 8.2996 & 0.0267 & 3404 & 8.4771 & 0.0533 \\
\hline 3192 & 7.9471 & Inf & 3263 & 8.1246 & Inf & 3334 & 8.3021 & 0.001 & 3405 & 8.4796 & Inf \\
\hline 3193 & 7.9496 & 0.16 & 3264 & 8.1271 & Inf & 3335 & 8.3046 & 0.0533 & 3406 & 8.4821 & Inf \\
\hline 3194 & 7.9521 & Inf & 3265 & 8.1296 & Inf & 3336 & 8.3071 & Inf & 3407 & 8.4846 & Inf \\
\hline 3195 & 7.9546 & Inf & 3266 & 8.1321 & Inf & 3337 & 8.3096 & Inf & 3408 & 8.4871 & Inf \\
\hline 3196 & 7.9571 & Inf & 3267 & 8.1346 & Inf & 3338 & 8.3121 & Inf & 3409 & 8.4896 & Inf \\
\hline 3197 & 7.9596 & Inf & 3268 & 8.1371 & Inf & 3339 & 8.3146 & Inf & 3410 & 8.4921 & Inf \\
\hline 3198 & 7.9621 & 0.0533 & 3269 & 8.1396 & Inf & 3340 & 8.3171 & Inf & 3411 & 8.4946 & Inf \\
\hline 3199 & 7.9646 & Inf & 3270 & 8.1421 & Inf & 3341 & 8.3196 & Inf & 3412 & 8.4971 & 0.16 \\
\hline 3200 & 7.9671 & Inf & 3271 & 8.1446 & Inf & 3342 & 8.3221 & Inf & 3413 & 8.4996 & 0.0133 \\
\hline 3201 & 7.9696 & Inf & 3272 & 8.1471 & Inf & 3343 & 8.3246 & Inf & 3414 & 8.5021 & 0.0004 \\
\hline 3202 & 7.9721 & Inf & 3273 & 8.1496 & Inf & 3344 & 8.3271 & 0.0533 & 3415 & 8.5046 & 0.0533 \\
\hline 3203 & 7.9746 & 0.0533 & 3274 & 8.1521 & Inf & 3345 & 8.3296 & Inf & 3416 & 8.5071 & Inf \\
\hline 3204 & 7.9771 & Inf & 3275 & 8.1546 & Inf & 3346 & 8.3321 & Inf & 3417 & 8.5096 & Inf \\
\hline 3205 & 7.9796 & 0.0533 & 3276 & 8.1571 & 0.16 & 3347 & 8.3346 & Inf & 3418 & 8.5121 & Inf \\
\hline
\end{tabular}




\begin{tabular}{|c|c|c|c|c|c|c|c|c|c|c|c|}
\hline 3419 & 8.5146 & Inf & 3490 & 8.6921 & Inf & 3561 & 8.8696 & Inf & 3632 & 9.0471 & Inf \\
\hline 3420 & 8.5171 & 0.16 & 3491 & 8.6946 & 0.04 & 3562 & 8.8721 & Inf & 3633 & 9.0496 & Inf \\
\hline 3421 & 8.5196 & Inf & 3492 & 8.6971 & $\operatorname{Inf}$ & 3563 & 8.8746 & 0.0533 & 3634 & 9.0521 & Inf \\
\hline 3422 & 8.5221 & Inf & 3493 & 8.6996 & 0.0267 & 3564 & 8.8771 & Inf & 3635 & 9.0546 & Inf \\
\hline 3423 & 8.5246 & Inf & 3494 & 8.7021 & 0.0003 & 3565 & 8.8796 & 0.0533 & 3636 & 9.0571 & Inf \\
\hline 3424 & 8.5271 & Inf & 3495 & 8.7046 & 0.0004 & 3566 & 8.8821 & Inf & 3637 & 9.0596 & Inf \\
\hline 3425 & 8.5296 & $\operatorname{Inf}$ & 3496 & 8.7071 & 0.0004 & 3567 & 8.8846 & Inf & 3638 & 9.0621 & Inf \\
\hline 3426 & 8.5321 & Inf & 3497 & 8.7096 & 0.0007 & 3568 & 8.8871 & Inf & 3639 & 9.0646 & Inf \\
\hline 3427 & 8.5346 & $\operatorname{Inf}$ & 3498 & 8.7121 & 0.04 & 3569 & 8.8896 & Inf & 3640 & 9.0671 & Inf \\
\hline 3428 & 8.5371 & 0.08 & 3499 & 8.7146 & Inf & 3570 & 8.8921 & Inf & 3641 & 9.0696 & Inf \\
\hline 3429 & 8.5396 & Inf & 3500 & 8.7171 & Inf & 3571 & 8.8946 & 0.0533 & 3642 & 9.0721 & 0.16 \\
\hline 3430 & 8.5421 & Inf & 3501 & 8.7196 & Inf & 3572 & 8.8971 & Inf & 3643 & 9.0746 & Inf \\
\hline 3431 & 8.5446 & Inf & 3502 & 8.7221 & Inf & 3573 & 8.8996 & 0.016 & 3644 & 9.0771 & Inf \\
\hline 3432 & 8.5471 & Inf & 3503 & 8.7246 & Inf & 3574 & 8.9021 & 0.0007 & 3645 & 9.0796 & Inf \\
\hline 3433 & 8.5496 & Inf & 3504 & 8.7271 & Inf & 3575 & 8.9046 & 0.04 & 3646 & 9.0821 & Inf \\
\hline 3434 & 8.5521 & $\operatorname{Inf}$ & 3505 & 8.7296 & Inf & 3576 & 8.9071 & 0.0533 & 3647 & 9.0846 & Inf \\
\hline 3435 & 8.5546 & Inf & 3506 & 8.7321 & Inf & 3577 & 8.9096 & Inf & 3648 & 9.0871 & 0.0533 \\
\hline 3436 & 8.5571 & Inf & 3507 & 8.7346 & Inf & 3578 & 8.9121 & 0.0533 & 3649 & 9.0896 & Inf \\
\hline 3437 & 8.5596 & 0.04 & 3508 & 8.7371 & Inf & 3579 & 8.9146 & Inf & 3650 & 9.0921 & Inf \\
\hline 3438 & 8.5621 & Inf & 3509 & 8.7396 & Inf & 3580 & 8.9171 & Inf & 3651 & 9.0946 & Inf \\
\hline 3439 & 8.5646 & Inf & 3510 & 8.7421 & Inf & 3581 & 8.9196 & Inf & 3652 & 9.0971 & Inf \\
\hline 3440 & 8.5671 & Inf & 3511 & 8.7446 & Inf & 3582 & 8.9221 & Inf & 3653 & 9.0996 & 0.0229 \\
\hline 3441 & 8.5696 & Inf & 3512 & 8.7471 & Inf & 3583 & 8.9246 & Inf & 3654 & 9.1021 & 0.0002 \\
\hline 3442 & 8.5721 & Inf & 3513 & 8.7496 & 0.16 & 3584 & 8.9271 & Inf & 3655 & 9.1046 & 0.0001 \\
\hline 3443 & 8.5746 & 0.08 & 3514 & 8.7521 & Inf & 3585 & 8.9296 & Inf & 3656 & 9.1071 & 0.0002 \\
\hline 3444 & 8.5771 & Inf & 3515 & 8.7546 & Inf & 3586 & 8.9321 & Inf & 3657 & 9.1096 & Inf \\
\hline 3445 & 8.5796 & 0.0533 & 3516 & 8.7571 & Inf & 3587 & 8.9346 & Inf & 3658 & 9.1121 & Inf \\
\hline 3446 & 8.5821 & Inf & 3517 & 8.7596 & Inf & 3588 & 8.9371 & Inf & 3659 & 9.1146 & Inf \\
\hline 3447 & 8.5846 & Inf & 3518 & 8.7621 & 0.08 & 3589 & 8.9396 & 0.16 & 3660 & 9.1171 & Inf \\
\hline 3448 & 8.5871 & Inf & 3519 & 8.7646 & Inf & 3590 & 8.9421 & Inf & 3661 & 9.1196 & Inf \\
\hline 3449 & 8.5896 & Inf & 3520 & 8.7671 & Inf & 3591 & 8.9446 & Inf & 3662 & 9.1221 & Inf \\
\hline 3450 & 8.5921 & 0.16 & 3521 & 8.7696 & Inf & 3592 & 8.9471 & Inf & 3663 & 9.1246 & Inf \\
\hline 3451 & 8.5946 & 0.0533 & 3522 & 8.7721 & Inf & 3593 & 8.9496 & Inf & 3664 & 9.1271 & Inf \\
\hline 3452 & 8.5971 & Inf & 3523 & 8.7746 & 0.0533 & 3594 & 8.9521 & Inf & 3665 & 9.1296 & Inf \\
\hline 3453 & 8.5996 & 0.0267 & 3524 & 8.7771 & Inf & 3595 & 8.9546 & Inf & 3666 & 9.1321 & Inf \\
\hline 3454 & 8.6021 & 0.001 & 3525 & 8.7796 & 0.04 & 3596 & 8.9571 & Inf & 3667 & 9.1346 & Inf \\
\hline 3455 & 8.6046 & 0.0533 & 3526 & 8.7821 & Inf & 3597 & 8.9596 & Inf & 3668 & 9.1371 & 0.0533 \\
\hline 3456 & 8.6071 & Inf & 3527 & 8.7846 & Inf & 3598 & 8.9621 & 0.0533 & 3669 & 9.1396 & 0.16 \\
\hline 3457 & 8.6096 & 0.16 & 3528 & 8.7871 & Inf & 3599 & 8.9646 & Inf & 3670 & 9.1421 & Inf \\
\hline 3458 & 8.6121 & Inf & 3529 & 8.7896 & Inf & 3600 & 8.9671 & Inf & 3671 & 9.1446 & Inf \\
\hline 3459 & 8.6146 & 0.0533 & 3530 & 8.7921 & Inf & 3601 & 8.9696 & Inf & 3672 & 9.1471 & Inf \\
\hline 3460 & 8.6171 & Inf & 3531 & 8.7946 & 0.0533 & 3602 & 8.9721 & Inf & 3673 & 9.1496 & Inf \\
\hline 3461 & 8.6196 & Inf & 3532 & 8.7971 & Inf & 3603 & 8.9746 & 0.04 & 3674 & 9.1521 & Inf \\
\hline 3462 & 8.6221 & Inf & 3533 & 8.7996 & Inf & 3604 & 8.9771 & Inf & 3675 & 9.1546 & Inf \\
\hline 3463 & 8.6246 & Inf & 3534 & 8.8021 & 0.0009 & 3605 & 8.9796 & 0.0533 & 3676 & 9.1571 & Inf \\
\hline 3464 & 8.6271 & Inf & 3535 & 8.8046 & 0.04 & 3606 & 8.9821 & Inf & 3677 & 9.1596 & 0.08 \\
\hline 3465 & 8.6296 & Inf & 3536 & 8.8071 & 0.0533 & 3607 & 8.9846 & Inf & 3678 & 9.1621 & Inf \\
\hline 3466 & 8.6321 & Inf & 3537 & 8.8096 & Inf & 3608 & 8.9871 & Inf & 3679 & 9.1646 & Inf \\
\hline 3467 & 8.6346 & Inf & 3538 & 8.8121 & 0.0533 & 3609 & 8.9896 & Inf & 3680 & 9.1671 & Inf \\
\hline 3468 & 8.6371 & Inf & 3539 & 8.8146 & Inf & 3610 & 8.9921 & Inf & 3681 & 9.1696 & Inf \\
\hline 3469 & 8.6396 & Inf & 3540 & 8.8171 & Inf & 3611 & 8.9946 & 0.0533 & 3682 & 9.1721 & Inf \\
\hline 3470 & 8.6421 & Inf & 3541 & 8.8196 & Inf & 3612 & 8.9971 & Inf & 3683 & 9.1746 & 0.04 \\
\hline 3471 & 8.6446 & Inf & 3542 & 8.8221 & Inf & 3613 & 8.9996 & 0.0533 & 3684 & 9.1771 & Inf \\
\hline 3472 & 8.6471 & Inf & 3543 & 8.8246 & Inf & 3614 & 9.0021 & 0.0007 & 3685 & 9.1796 & 0.0533 \\
\hline 3473 & 8.6496 & Inf & 3544 & 8.8271 & Inf & 3615 & 9.0046 & 0.0533 & 3686 & 9.1821 & Inf \\
\hline 3474 & 8.6521 & Inf & 3545 & 8.8296 & Inf & 3616 & 9.0071 & Inf & 3687 & 9.1846 & Inf \\
\hline 3475 & 8.6546 & Inf & 3546 & 8.8321 & Inf & 3617 & 9.0096 & 0.0533 & 3688 & 9.1871 & Inf \\
\hline 3476 & 8.6571 & Inf & 3547 & 8.8346 & Inf & 3618 & 9.0121 & Inf & 3689 & 9.1896 & Inf \\
\hline 3477 & 8.6596 & Inf & 3548 & 8.8371 & 0.16 & 3619 & 9.0146 & Inf & 3690 & 9.1921 & Inf \\
\hline 3478 & 8.6621 & 0.16 & 3549 & 8.8396 & Inf & 3620 & 9.0171 & Inf & 3691 & 9.1946 & 0.0533 \\
\hline 3479 & 8.6646 & 0.0533 & 3550 & 8.8421 & Inf & 3621 & 9.0196 & Inf & 3692 & 9.1971 & Inf \\
\hline 3480 & 8.6671 & $\operatorname{Inf}$ & 3551 & 8.8446 & Inf & 3622 & 9.0221 & Inf & 3693 & 9.1996 & Inf \\
\hline 3481 & 8.6696 & Inf & 3552 & 8.8471 & Inf & 3623 & 9.0246 & Inf & 3694 & 9.2021 & 0.0007 \\
\hline 3482 & 8.6721 & Inf & 3553 & 8.8496 & Inf & 3624 & 9.0271 & Inf & 3695 & 9.2046 & 0.0533 \\
\hline 3483 & 8.6746 & Inf & 3554 & 8.8521 & Inf & 3625 & 9.0296 & Inf & 3696 & 9.2071 & Inf \\
\hline 3484 & 8.6771 & Inf & 3555 & 8.8546 & Inf & 3626 & 9.0321 & 0.04 & 3697 & 9.2096 & Inf \\
\hline 3485 & 8.6796 & 0.0533 & 3556 & 8.8571 & Inf & 3627 & 9.0346 & Inf & 3698 & 9.2121 & Inf \\
\hline 3486 & 8.6821 & Inf & 3557 & 8.8596 & Inf & 3628 & 9.0371 & 0.0533 & 3699 & 9.2146 & 0.04 \\
\hline 3487 & 8.6846 & Inf & 3558 & 8.8621 & 0.0533 & 3629 & 9.0396 & Inf & 3700 & 9.2171 & Inf \\
\hline 3488 & 8.6871 & Inf & 3559 & 8.8646 & Inf & 3630 & 9.0421 & Inf & 3701 & 9.2196 & Inf \\
\hline 3489 & 8.6896 & Inf & 3560 & 8.8671 & Inf & 3631 & 9.0446 & Inf & 3702 & 9.2221 & Inf \\
\hline
\end{tabular}




\begin{tabular}{|c|c|c|c|c|c|c|c|c|c|c|c|}
\hline 3703 & 9.2246 & Inf & 3774 & 9.4021 & 0.0007 & 3845 & 9.5796 & 0.04 & 3916 & 9.7571 & Inf \\
\hline 3704 & 9.2271 & Inf & 3775 & 9.4046 & 0.0533 & 3846 & 9.5821 & Inf & 3917 & 9.7596 & Inf \\
\hline 3705 & 9.2296 & Inf & 3776 & 9.4071 & Inf & 3847 & 9.5846 & Inf & 3918 & 9.7621 & 0.0533 \\
\hline 3706 & 9.2321 & Inf & 3777 & 9.4096 & Inf & 3848 & 9.5871 & Inf & 3919 & 9.7646 & Inf \\
\hline 3707 & 9.2346 & Inf & 3778 & 9.4121 & Inf & 3849 & 9.5896 & Inf & 3920 & 9.7671 & Inf \\
\hline 3708 & 9.2371 & Inf & 3779 & 9.4146 & 0.04 & 3850 & 9.5921 & Inf & 3921 & 9.7696 & Inf \\
\hline 3709 & 9.2396 & Inf & 3780 & 9.4171 & Inf & 3851 & 9.5946 & 0.0533 & 3922 & 9.7721 & Inf \\
\hline 3710 & 9.2421 & Inf & 3781 & 9.4196 & Inf & 3852 & 9.5971 & Inf & 3923 & 9.7746 & 0.04 \\
\hline 3711 & 9.2446 & $\operatorname{Inf}$ & 3782 & 9.4221 & Inf & 3853 & 9.5996 & 0.0533 & 3924 & 9.7771 & Inf \\
\hline 3712 & 9.2471 & Inf & 3783 & 9.4246 & Inf & 3854 & 9.6021 & 0.0008 & 3925 & 9.7796 & 0.0533 \\
\hline 3713 & 9.2496 & Inf & 3784 & 9.4271 & Inf & 3855 & 9.6046 & 0.0533 & 3926 & 9.7821 & Inf \\
\hline 3714 & 9.2521 & Inf & 3785 & 9.4296 & Inf & 3856 & 9.6071 & 0.0533 & 3927 & 9.7846 & Inf \\
\hline 3715 & 9.2546 & Inf & 3786 & 9.4321 & Inf & 3857 & 9.6096 & Inf & 3928 & 9.7871 & Inf \\
\hline 3716 & 9.2571 & Inf & 3787 & 9.4346 & Inf & 3858 & 9.6121 & 0.0533 & 3929 & 9.7896 & Inf \\
\hline 3717 & 9.2596 & 0.16 & 3788 & 9.4371 & Inf & 3859 & 9.6146 & Inf & 3930 & 9.7921 & Inf \\
\hline 3718 & 9.2621 & $\operatorname{Inf}$ & 3789 & 9.4396 & Inf & 3860 & 9.6171 & Inf & 3931 & 9.7946 & 0.0533 \\
\hline 3719 & 9.2646 & 0.0533 & 3790 & 9.4421 & Inf & 3861 & 9.6196 & Inf & 3932 & 9.7971 & Inf \\
\hline 3720 & 9.2671 & Inf & 3791 & 9.4446 & Inf & 3862 & 9.6221 & Inf & 3933 & 9.7996 & 0.0178 \\
\hline 3721 & 9.2696 & Inf & 3792 & 9.4471 & Inf & 3863 & 9.6246 & Inf & 3934 & 9.8021 & 0.0008 \\
\hline 3722 & 9.2721 & Inf & 3793 & 9.4496 & Inf & 3864 & 9.6271 & 0.16 & 3935 & 9.8046 & 0.0034 \\
\hline 3723 & 9.2746 & Inf & 3794 & 9.4521 & Inf & 3865 & 9.6296 & Inf & 3936 & 9.8071 & 0.0035 \\
\hline 3724 & 9.2771 & 0.0533 & 3795 & 9.4546 & Inf & 3866 & 9.6321 & Inf & 3937 & 9.8096 & 0.0033 \\
\hline 3725 & 9.2796 & Inf & 3796 & 9.4571 & Inf & 3867 & 9.6346 & Inf & 3938 & 9.8121 & 0.0044 \\
\hline 3726 & 9.2821 & 0.0533 & 3797 & 9.4596 & 0.16 & 3868 & 9.6371 & Inf & 3939 & 9.8146 & Inf \\
\hline 3727 & 9.2846 & Inf & 3798 & 9.4621 & Inf & 3869 & 9.6396 & Inf & 3940 & 9.8171 & Inf \\
\hline 3728 & 9.2871 & Inf & 3799 & 9.4646 & 0.0533 & 3870 & 9.6421 & Inf & 3941 & 9.8196 & Inf \\
\hline 3729 & 9.2896 & Inf & 3800 & 9.4671 & Inf & 3871 & 9.6446 & Inf & 3942 & 9.8221 & Inf \\
\hline 3730 & 9.2921 & 0.16 & 3801 & 9.4696 & Inf & 3872 & 9.6471 & Inf & 3943 & 9.8246 & Inf \\
\hline 3731 & 9.2946 & Inf & 3802 & 9.4721 & Inf & 3873 & 9.6496 & Inf & 3944 & 9.8271 & Inf \\
\hline 3732 & 9.2971 & 0.0533 & 3803 & 9.4746 & Inf & 3874 & 9.6521 & Inf & 3945 & 9.8296 & 0.0533 \\
\hline 3733 & 9.2996 & 0.0533 & 3804 & 9.4771 & Inf & 3875 & 9.6546 & Inf & 3946 & 9.8321 & 0.16 \\
\hline 3734 & 9.3021 & 0.0002 & 3805 & 9.4796 & 0.08 & 3876 & 9.6571 & Inf & 3947 & 9.8346 & 0.0533 \\
\hline 3735 & 9.3046 & 0.0007 & 3806 & 9.4821 & Inf & 3877 & 9.6596 & Inf & 3948 & 9.8371 & Inf \\
\hline 3736 & 9.3071 & Inf & 3807 & 9.4846 & Inf & 3878 & 9.6621 & 0.0533 & 3949 & 9.8396 & Inf \\
\hline 3737 & 9.3096 & Inf & 3808 & 9.4871 & Inf & 3879 & 9.6646 & 0.16 & 3950 & 9.8421 & Inf \\
\hline 3738 & 9.3121 & Inf & 3809 & 9.4896 & Inf & 3880 & 9.6671 & Inf & 3951 & 9.8446 & Inf \\
\hline 3739 & 9.3146 & 0.04 & 3810 & 9.4921 & Inf & 3881 & 9.6696 & Inf & 3952 & 9.8471 & Inf \\
\hline 3740 & 9.3171 & Inf & 3811 & 9.4946 & 0.04 & 3882 & 9.6721 & $\operatorname{Inf}$ & 3953 & 9.8496 & Inf \\
\hline 3741 & 9.3196 & Inf & 3812 & 9.4971 & Inf & 3883 & 9.6746 & 0.0533 & 3954 & 9.8521 & Inf \\
\hline 3742 & 9.3221 & Inf & 3813 & 9.4996 & 0.0267 & 3884 & 9.6771 & Inf & 3955 & 9.8546 & Inf \\
\hline 3743 & 9.3246 & Inf & 3814 & 9.5021 & 0.0004 & 3885 & 9.6796 & 0.0533 & 3956 & 9.8571 & Inf \\
\hline 3744 & 9.3271 & Inf & 3815 & 9.5046 & 0.04 & 3886 & 9.6821 & Inf & 3957 & 9.8596 & Inf \\
\hline 3745 & 9.3296 & Inf & 3816 & 9.5071 & Inf & 3887 & 9.6846 & Inf & 3958 & 9.8621 & Inf \\
\hline 3746 & 9.3321 & Inf & 3817 & 9.5096 & Inf & 3888 & 9.6871 & Inf & 3959 & 9.8646 & Inf \\
\hline 3747 & 9.3346 & Inf & 3818 & 9.5121 & Inf & 3889 & 9.6896 & Inf & 3960 & 9.8671 & Inf \\
\hline 3748 & 9.3371 & Inf & 3819 & 9.5146 & Inf & 3890 & 9.6921 & Inf & 3961 & 9.8696 & Inf \\
\hline 3749 & 9.3396 & Inf & 3820 & 9.5171 & Inf & 3891 & 9.6946 & 0.0533 & 3962 & 9.8721 & Inf \\
\hline 3750 & 9.3421 & Inf & 3821 & 9.5196 & Inf & 3892 & 9.6971 & Inf & 3963 & 9.8746 & Inf \\
\hline 3751 & 9.3446 & Inf & 3822 & 9.5221 & Inf & 3893 & 9.6996 & 0.0267 & 3964 & 9.8771 & 0.16 \\
\hline 3752 & 9.3471 & Inf & 3823 & 9.5246 & Inf & 3894 & 9.7021 & 0.001 & 3965 & 9.8796 & Inf \\
\hline 3753 & 9.3496 & Inf & 3824 & 9.5271 & 0.0533 & 3895 & 9.7046 & 0.0533 & 3966 & 9.8821 & Inf \\
\hline 3754 & 9.3521 & Inf & 3825 & 9.5296 & Inf & 3896 & 9.7071 & 0.0533 & 3967 & 9.8846 & 0.0533 \\
\hline 3755 & 9.3546 & Inf & 3826 & 9.5321 & Inf & 3897 & 9.7096 & Inf & 3968 & 9.8871 & Inf \\
\hline 3756 & 9.3571 & Inf & 3827 & 9.5346 & Inf & 3898 & 9.7121 & 0.0533 & 3969 & 9.8896 & Inf \\
\hline 3757 & 9.3596 & 0.16 & 3828 & 9.5371 & Inf & 3899 & 9.7146 & Inf & 3970 & 9.8921 & Inf \\
\hline 3758 & 9.3621 & Inf & 3829 & 9.5396 & Inf & 3900 & 9.7171 & Inf & 3971 & 9.8946 & 0.0533 \\
\hline 3759 & 9.3646 & 0.0533 & 3830 & 9.5421 & Inf & 3901 & 9.7196 & Inf & 3972 & 9.8971 & 0.16 \\
\hline 3760 & 9.3671 & Inf & 3831 & 9.5446 & 0.16 & 3902 & 9.7221 & Inf & 3973 & 9.8996 & 0.0229 \\
\hline 3761 & 9.3696 & Inf & 3832 & 9.5471 & Inf & 3903 & 9.7246 & Inf & 3974 & 9.9021 & 0.0008 \\
\hline 3762 & 9.3721 & Inf & 3833 & 9.5496 & Inf & 3904 & 9.7271 & Inf & 3975 & 9.9046 & 0.016 \\
\hline 3763 & 9.3746 & Inf & 3834 & 9.5521 & Inf & 3905 & 9.7296 & Inf & 3976 & 9.9071 & Inf \\
\hline 3764 & 9.3771 & $\operatorname{Inf}$ & 3835 & 9.5546 & Inf & 3906 & 9.7321 & Inf & 3977 & 9.9096 & Inf \\
\hline 3765 & 9.3796 & 0.0533 & 3836 & 9.5571 & Inf & 3907 & 9.7346 & Inf & 3978 & 9.9121 & Inf \\
\hline 3766 & 9.3821 & Inf & 3837 & 9.5596 & Inf & 3908 & 9.7371 & Inf & 3979 & 9.9146 & Inf \\
\hline 3767 & 9.3846 & 0.16 & 3838 & 9.5621 & Inf & 3909 & 9.7396 & 0.16 & 3980 & 9.9171 & Inf \\
\hline 3768 & 9.3871 & Inf & 3839 & 9.5646 & Inf & 3910 & 9.7421 & Inf & 3981 & 9.9196 & Inf \\
\hline 3769 & 9.3896 & Inf & 3840 & 9.5671 & Inf & 3911 & 9.7446 & Inf & 3982 & 9.9221 & Inf \\
\hline 3770 & 9.3921 & $\operatorname{Inf}$ & 3841 & 9.5696 & Inf & 3912 & 9.7471 & Inf & 3983 & 9.9246 & Inf \\
\hline 3771 & 9.3946 & 0.0533 & 3842 & 9.5721 & Inf & 3913 & 9.7496 & Inf & 3984 & 9.9271 & 0.04 \\
\hline 3772 & 9.3971 & Inf & 3843 & 9.5746 & 0.0533 & 3914 & 9.7521 & Inf & 3985 & 9.9296 & Inf \\
\hline 3773 & 9.3996 & 0.0267 & 3844 & 9.5771 & $\operatorname{Inf}$ & 3915 & 9.7546 & Inf & 3986 & 9.9321 & Inf \\
\hline
\end{tabular}




\begin{tabular}{|c|c|c|c|c|c|c|c|c|c|c|c|}
\hline 3987 & 9.9346 & Inf & 3994 & 9.9521 & Inf & 4001 & 9.9696 & Inf & 4008 & 9.9871 & Inf \\
\hline 3988 & 9.9371 & Inf & 3995 & 9.9546 & Inf & 4002 & 9.9721 & 0.16 & 4009 & 9.9896 & Inf \\
\hline 3989 & 9.9396 & Inf & 3996 & 9.9571 & Inf & 4003 & 9.9746 & Inf & 4010 & 9.9921 & Inf \\
\hline 3990 & 9.9421 & Inf & 3997 & 9.9596 & Inf & 4004 & 9.9771 & 0.0533 & 4011 & 9.9946 & Inf \\
\hline 3991 & 9.9446 & Inf & 3998 & 9.9621 & Inf & 4005 & 9.9796 & Inf & 4012 & 9.9971 & Inf \\
\hline 3992 & 9.9471 & Inf & 3999 & 9.9646 & Inf & 4006 & 9.9821 & Inf & 4013 & 9.9996 & 0.0114 \\
\hline 3993 & 9.9496 & Inf & 4000 & 9.9671 & Inf & 4007 & 9.9846 & Inf & 4014 & 10.002 & 0.0001 \\
\hline
\end{tabular}

